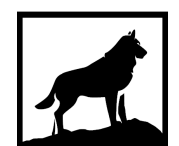

Michigan Technological

1 8 8 5 University
Michigan Technological University

Digital Commons @ Michigan Tech

Dust Susceptibility at Mine Tailings Impoundments: Thermal Remote Sensing for Dust Susceptibility Characterization and Biological Soil Crusts for Dust Susceptibility Reduction

Bonnie Zwissler

Michigan Technological University, bezwissl@mtu.edu

Copyright 2016 Bonnie Zwissler

Recommended Citation

Zwissler, Bonnie, "Dust Susceptibility at Mine Tailings Impoundments: Thermal Remote Sensing for Dust Susceptibility Characterization and Biological Soil Crusts for Dust Susceptibility Reduction", Open Access Dissertation, Michigan Technological University, 2016.

https://doi.org/10.37099/mtu.dc.etdr/309

Follow this and additional works at: https://digitalcommons.mtu.edu/etdr

Part of the Geotechnical Engineering Commons 


\title{
DUST SUSCEPTIBILITY AT MINE TAILINGS IMPOUNDMENTS: THERMAL REMOTE SENSING FOR DUST SUSCEPTIBLITY CHARACTERIZATION AND BIOLOGICAL SOIL CRUSTS FOR DUST SUSCEPTIBILITY REDUCTION
}

By

Bonnie E. Zwissler

\begin{abstract}
A DISSERTATION
Submitted in partial fulfillment of the requirements for the degree of DOCTOR OF PHILOSOPHY

In Civil Engineering
\end{abstract}

MICHIGAN TECHNOLOGICAL UNIVERSITY

2016

(C) 2016 Bonnie E. Zwissler 

This dissertation has been approved in partial fulfillment of the requirements for the Degree of DOCTOR OF PHILOSOPHY in Civil Engineering.

Department of Civil and Environmental Engineering

Dissertation Co-Advisor: Thomas Oommen

Dissertation Co-Advisor: $\quad$ Eric Seagren

Committee Member: Stan Vitton

Committee Member: Ann Maclean

Department Chair: Dave Hand 



\section{Table of Contents}

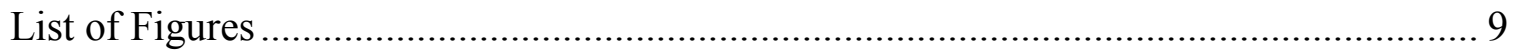

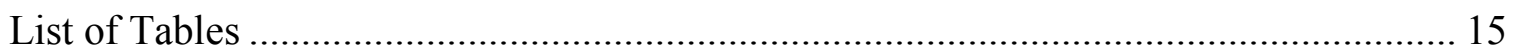

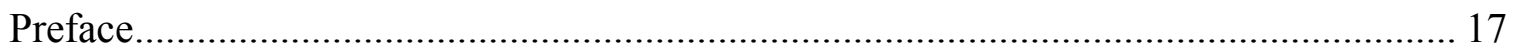

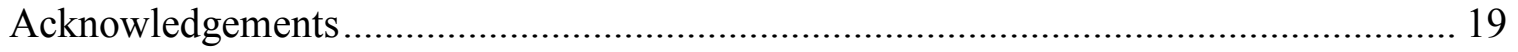

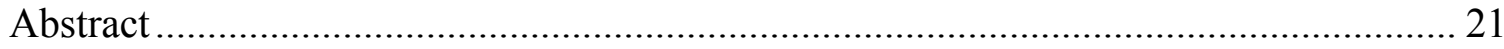

Chapter 1 Introduction, Objectives, and Literature Review ........................................ 23

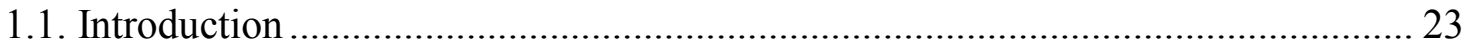

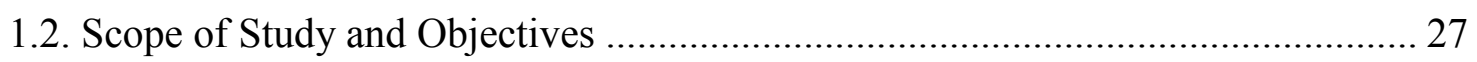

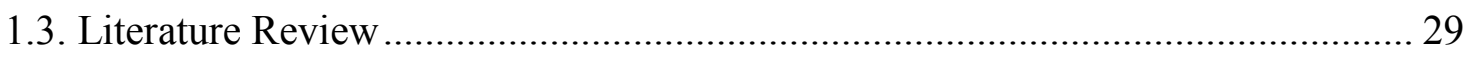

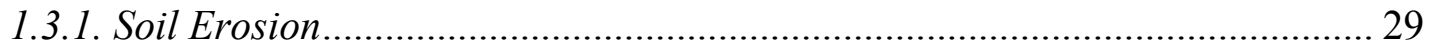

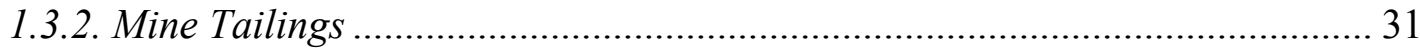

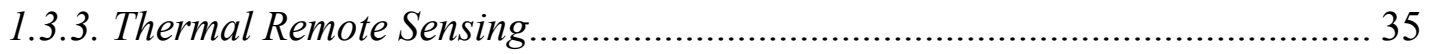

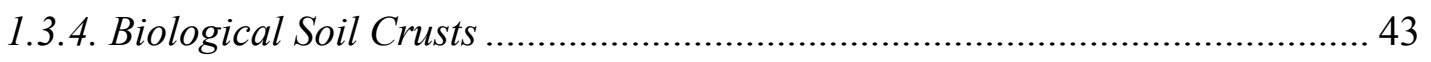

1.3.5. Surficial Strength and Erosion Susceptibility Testing ................................ 48

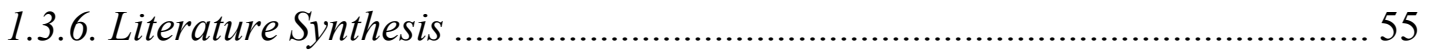

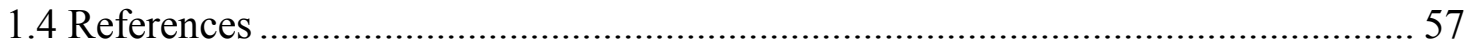

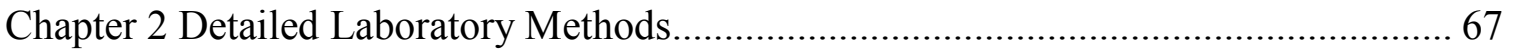

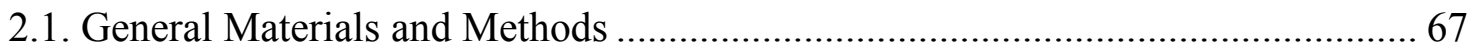

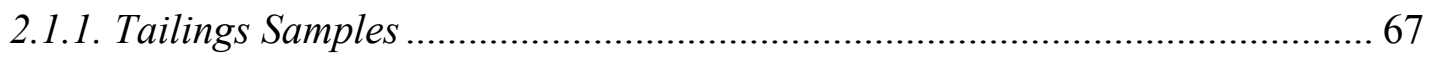

2.1.2. Preparation of Laboratory Specimens …................................................. 75

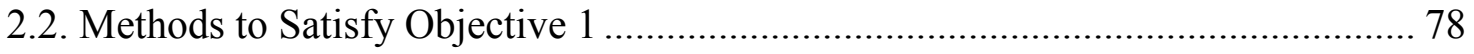

2.2.1. Laboratory testing of thermal properties, moisture content, and strength .... 78

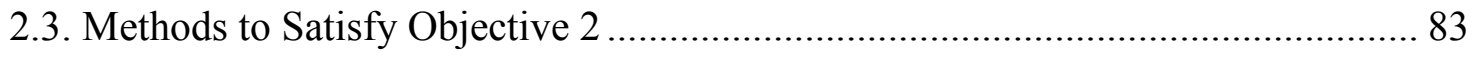

2.3.1. Collection of Thermal Images and Ground Truth Data ............................... 83

2.3.2. Proof-of-Concept Satellite and UAV Imagery Assessment ............................ 87

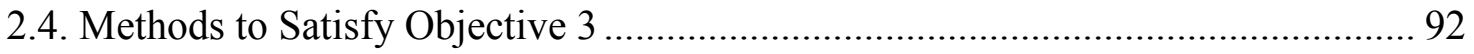

2.4.1. Sublimation of Laboratory Specimens ....................................................... 92

2.4.2. Wind Tunnel Testing of Laboratory Specimens ......................................... 100

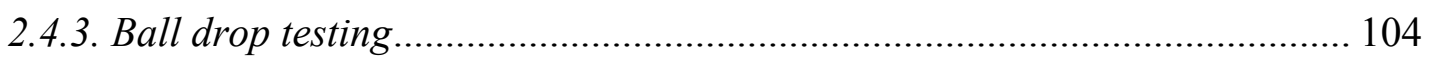

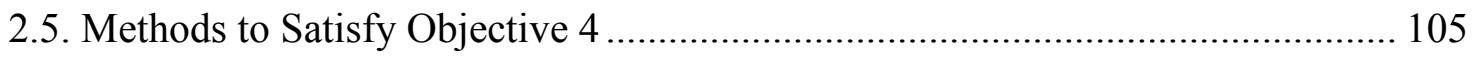


2.5.2. Biological Soil Crusts organism selection .................................................... 106

2.5.3. Bioreactor Inoculation and Maintenance .................................................. 110

2.5.4. Biomass Characterization of Biological Soil Crusts .................................... 115

2.5.5. Wind Erosion Resistance Testing of Biological Soil Crusts ......................... 121

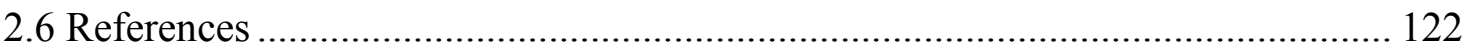

Chapter 3 Thermal remote sensing for moisture content of mine tailings: laboratory study

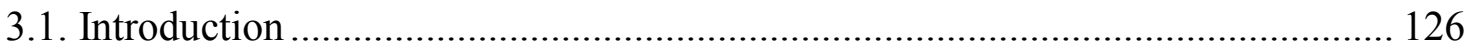

3.2. Existing Techniques Utilizing Remote Sensing to Detect Moisture Content ...... 129

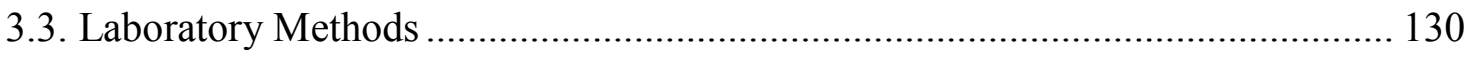

3.4. Laboratory Results ................................................................................... 135

3.5. Multivariate Regression Model Results and Discussion...................................... 141

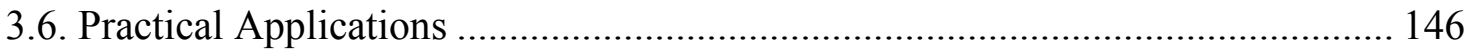

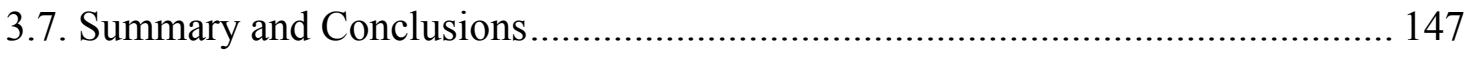

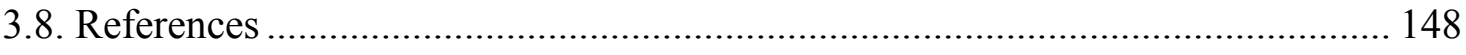

Chapter 4 Thermal remote sensing for moisture content of mine tailings: field study... 153

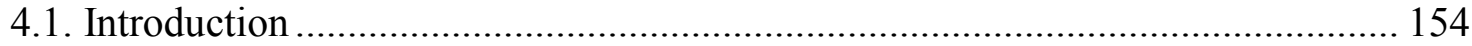

4.2. Description of Laboratory Model................................................................ 156

4.3. Application of Laboratory Model to Field Scale ………………....................... 158

4.4. Results and Discussion of Field Testing .......................................................... 162

4.5. Future Work Required for Effective Field Application ...................................... 169

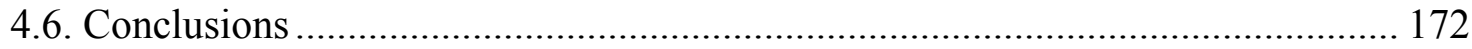

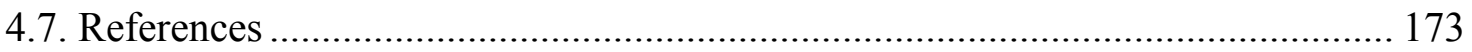

Chapter 5 Laboratory analysis of the generation and abatement of dust events on mine

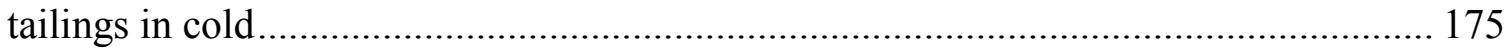

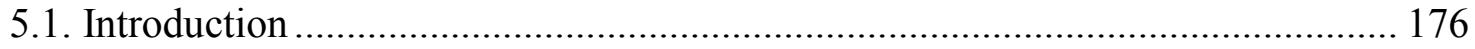

5.2. Cold Weather Dusting ..................................................................................... 179

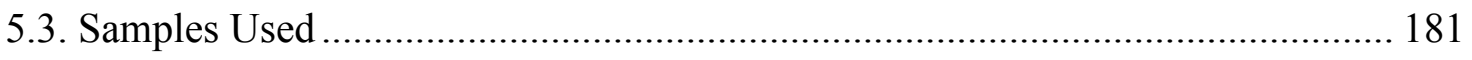

5.4. Field Observations of Sublimation and Associated Cold Weather Dust Events.. 183

5.5. Laboratory Methods to Generate Sublimation and Dust........................................ 186 


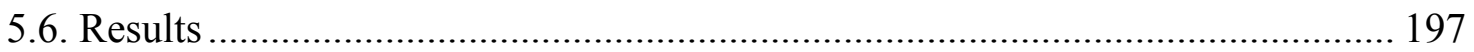

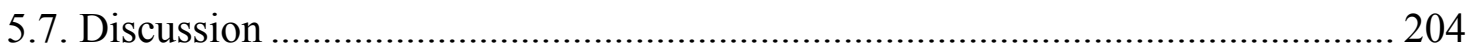

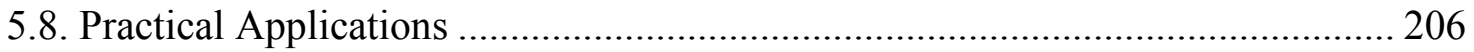

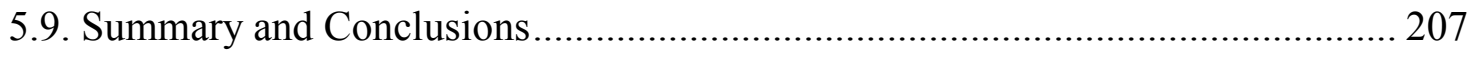

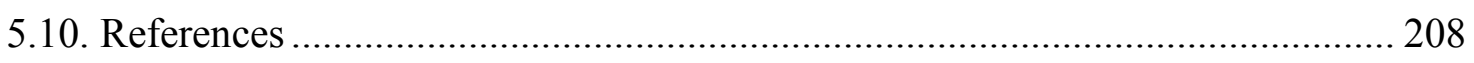

Chapter 6 Biological Soil Crust monitoring and laboratory testing to explore dust

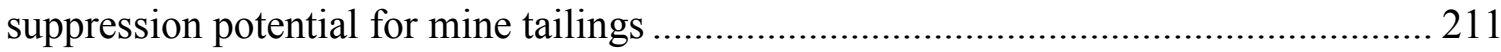

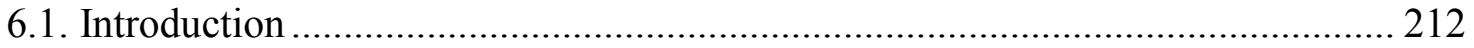

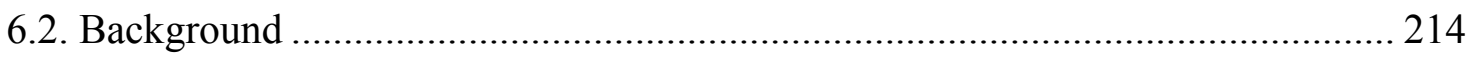

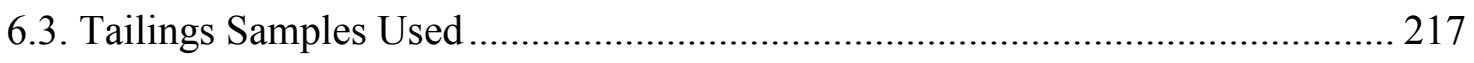

6.4. Proof-of-Concept Growth of BSCs on tailings ................................................ 217

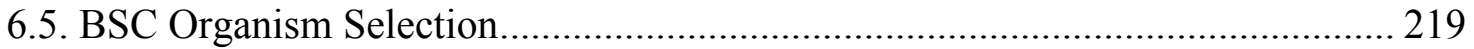

6.6. Methods for Bioreactor Inoculation, Treatment, Monitoring, Biomass Characterization, and Strength Increase Methods ..................................................... 221

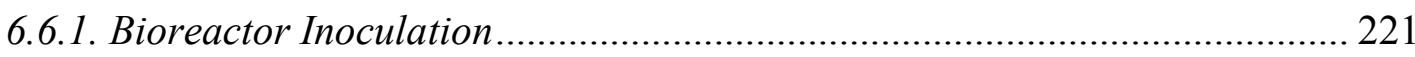

6.6.2. Bioreactor Treatment and Monitoring....................................................... 225

6.6.3. Biomass Characterization.................................................................... 226

6.6.4. Scanning Electron Microscopy .................................................................... 229

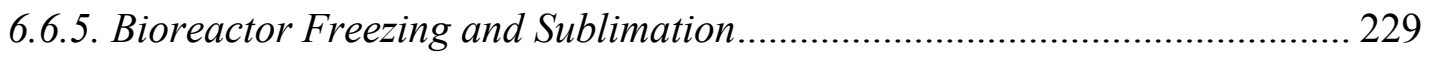

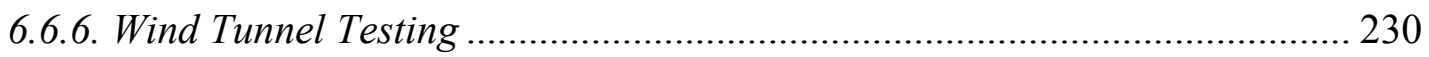

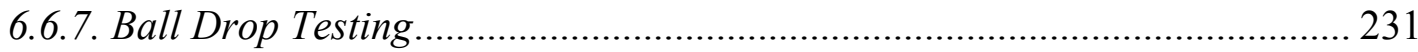

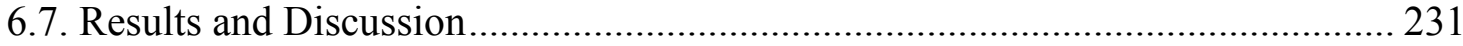

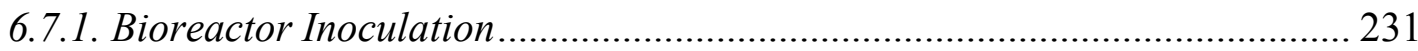

6.7.2. Bioreactor Treatment and Monitoring...................................................... 232

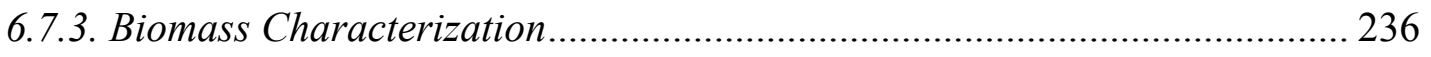

6.7.4. Scanning Electron Microscopy …………………………........................... 240

6.7.5. Bioreactor Freezing and Sublimation..................................................... 243

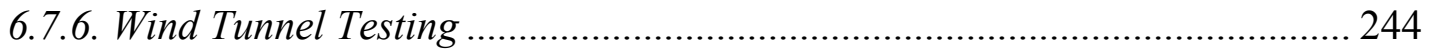

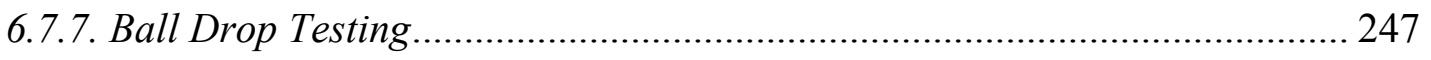

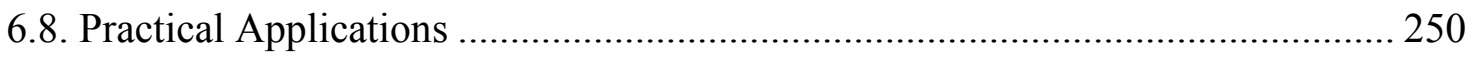

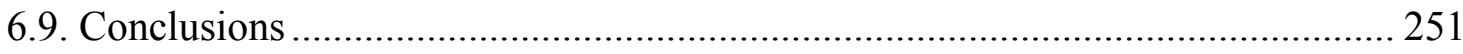




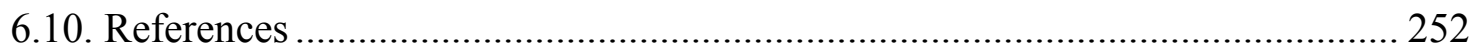

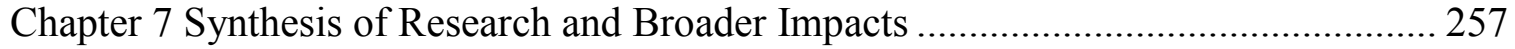

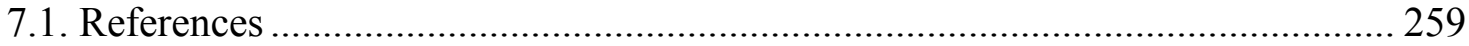




\section{List of Figures}

Figure 2-1 | Study site map for Empire and Gribben Mine Tailings Impoundments in Marquette County, MI.

Figure 2-2 | Study site map for United Taconite Mine Tailings Impoundments in St. Louis County, MN.

Figure 2-3 | Grain size distributions for MI-mag and MN-mag samples used in laboratory testing.

Figure 2-4 | Photo showing rotary sample splitter used for blending of MI-mag and MNmag samples used in Objectives 3 and 4: (a) rotary sample splitter, showing use of snorkel ventilation system, hopper, vibratory conveyer belt, and twelve sample bins on rotating platform; (b) closer view of vibratory conveyer belt and rotating platform, showing sample being divided into twelve equal parts.

Figure 2-5 | Schematic of soil box packing technique, used in the preparation of all laboratory samples. 77

Figure 2-6 | Schematic of Mariotte Tube, used for saturation of tailings samples. ......... 77

Figure 2-7 | Schematic of laboratory thermal remote sensing testing set-up.

Figure 2-8 | Sample temperature determination using FLIR ThermaCam Researcher Professional software using thermal images for MN-Mag sample tested on June 4, 2015: (a) object parameter entry for pre-heating conditions, (b) object parameter entry for postheating conditions, (c) thermal image representing surface temperature for pre-heating sample, and (d) thermal image representing surface temperature for post-heating sample. Using the pre- and post- heating thermal images (c and d), the average surface temperature for the square sample was determined and used to calculate change in surface temperature (average pre-heating sample temperature from (c) of $21.4^{\circ} \mathrm{C}$ or 294.6 $\mathrm{K}$, average post-heating sample temperature from (d) of $31.8^{\circ} \mathrm{C}$ or $305.0 \mathrm{~K}, \Delta \mathrm{T}$ of 10.4 $\mathrm{K})$

Figure 2-9 | Procedure used to process ASTER data to obtain $\Delta T$.

Figure 2-10 | Spatial variation in $\Delta T$ observed using ASTER data: (top) map of $\Delta T$ calculated using two ASTER scenes (April 23 and July 3, 2002), showing variation in $\Delta \mathrm{T}$ can be detected; (bottom) real-color image showing impoundment at time of ASTER acquisition, showing water pond in center and unsaturated tailings on the east and west sides (data source: Landsat 7, image date July 28, 2002).

Figure 2-11 | Thermal infrared imagery (left) and visible imagery (right) of MN-mag tailings impoundment collected with UAV in May 2015. In the thermal imagery, dark colors represent lower relative temperatures, and bright colors represent higher relative temperatures. Detail including tire tracks can be distinguished in both images.

Figure 2-12 | Schematic of freezing/sublimation setup for MI-mag and MN-mag samples, with (top) plan view and (bottom) front view 96 
Figure 2-13 | Laboratory MI-mag tailings samples after freezing/sublimation, showing ice lens formation and structure. Small ice lenses are visible throughout samples, and large ice lenses are marked with red circles.....

Figure 2-14 | Frozen field MI-mag tailings samples, showing ice lens formation and structure: (a) and (c) show ice lens structure on the tailings surface, (b) shows ice lens structure with depth as seen from the side, and (d) shows ice lenses structure in-situ..... 99

Figure 2-15 | Frozen field MN-mag tailings samples, showing ice lens formation and structure.

Figure 2-16 | Wind tunnel used at Michigan Technological University, Houghton, MI.103

Figure 2-17 | Wind speed calibration curve for wind tunnel based on average wind speed over 30 seconds at sample location with sandpaper base.

Figure 2-18 | Biological soil crusts growing on MI-mag (left) and MI-hem (right) tailings surface.

Figure 2-19 | Eagle River crust growing on MI-hem tailings (left) and viewed under light microscope at 40x magnification (right). It should be noted that the MI-hem tailings are naturally red, and the crust is black, showing almost total coverage of tailings..... 109

Figure 2-20 | Schematic of bioreactor inoculation, where one batch of $400 \mathrm{~mL}$ inoculum was divided evenly between 6 soil boxes, featuring removable collars to provide extra headspace in which the inoculum could reside until percolating through the tailings sample.

Figure 2-21 | Glucose standard curve used to determine polysaccharide concentration in biological soil crusts samples.

Figure 2-22 | Chlorophyll a standard curve used to determine chlorophyll a concentration in biological soil crusts samples.

Figure 3-1 | Hazards associated with surface strength at mine tailings impoundments: (a) fugitive dust emissions, and (b) trafficability issues.

Figure 3-2 | Schematic of laboratory thermal remote sensing testing set-up, featuring two halogen lamps and 2 full-spectrum lamps mounted at 45 degree angles from iron samples.

Figure 3-3 | Thermal images collected for a MN-mag sample on June 8, 2015: (a) preheating, with a measured sample moisture content of 8.6 percent, a measured sample penetration depth of $0.40 \mathrm{~cm}$, and an average sample temperature of $295.1 \mathrm{~K}$; and (b) post-heating, with a measured sample moisture content of 7.3 percent, a measured sample penetration depth of $0.35 \mathrm{~cm}$, and an average sample temperature of $306.8 \mathrm{~K} \ldots \ldots \ldots \ldots . . . .135$

Figure 3-4 | Relationship between moisture content and surface strength (penetration depth) for MI-mag tailings. Each data point represents the daily average measurement for a sample. 
Figure 3-5 | Relationship between moisture content and surface strength (penetration depth) for MN-mag tailings. Each data point represents the daily average measurement for a sample.

Figure 3-6 | Atmospheric conditions during laboratory testing of MI-mag tailings. Each data point represents a single measurement.

Figure 3-7 | Atmospheric conditions during laboratory testing of MN-mag tailings. Each data point represents a single measurement. 141

Figure 3-8 | Multivariate linear regression validation to predict moisture content for MImag tailings with thermal remote sensing and atmospheric variables. Points indicate daily average values for each sample, and the line shown is the 1:1 line for the data. 145

Figure 3-9 | Multivariate linear regression validation to predict moisture content for MNmag tailings with thermal remote sensing and atmospheric variables. Points indicate daily average values for each sample, and the line shown is the 1:1 line for the data. 146

Figure 4-1 | Site marking and testing scheme used for fifteen study sites at MI-mag tailings impoundment in June 2016.

Figure 4-2 | Thermal infrared imagery of MI-mag tailings impoundment collected (left) pre-heating, and (right) post-heating, in June 2016. Dark colors represent lower relative temperatures, and bright colors represent higher relative temperatures. Average temperature was determined for the tailings inside the $0.3 \mathrm{~m} \mathrm{x} 0.3 \mathrm{~m}$ spray paint target.

Figure 4-3 | Relationship between moisture content and surface temperature for fifteen study sites at MI-mag tailings impoundment in June $2016\left(\mathrm{R}^{2}=0.80\right)$. 166

Figure 4-4 | Relationship between moisture content and surface temperature for laboratory MI-mag tailings samples tested in summer $2014\left(\mathrm{R}^{2}=0.25\right)$. Each point represents the daily average value for a single sample.

Figure 4-5 | Relationship between moisture content and relative surface strength for MImag tailings. Laboratory strength data $(n=116)$ were measured using a needle penetrometer, where penetration depth represents the inverse of relative strength. Field strength data $(n=30)$ were measured using a vane shear device, where degrees of rotation represents relative strength. Each point represents a single measurement. 168

Figure 4-6 | Model validation for using laboratory-developed model for field data $(n=12)$ from MI-mag tailings collected in June 2016. Each point represents data from a single site.

Figure 4-7 | Thermal infrared imagery of MN-mag tailings impoundment collected with UAV in May 2015. Dark colors represent lower relative temperatures, and bright colors represent higher relative temperatures. Detail including tire tracks can be distinguished in this imagery (left). 172

Figure 5-1 | Grain size distributions for tailings samples used in laboratory testing..... 183 
Figure 5-2 | Frozen field MI-mag tailings samples, showing ice lens formation and structure

Figure 5-3 | Frozen field MN-mag tailings samples, showing ice lens formation and structure.

Figure 5-4 | Schematic of soil box packing technique, used in the preparation of all laboratory samples.

Figure 5-5 | Schematic of Mariotte Tube, used for saturation of tailings samples. 188

Figure 5-6 | Schematic (top view) of freezing/sublimation setup for MI-mag and MN-mag samples.

Figure 5-7 | Schematic (front view) of freezing/sublimation setup for MI-mag and MNmag samples.

Figure 5-8 | Wind tunnel located at Michigan Technological University, Houghton, MI.

Figure 5-9 | Thermocouple data for MI/MN blend tailings sample exposed to 7 days of freezing, verifying that sample freezing occurred from the top down.

Figure 5-10 | Laboratory MI/MN blend tailings samples after freezing/sublimation, showing ice lens orientation and structure: a) and b) show vertically oriented ice lenses, and c) and d) show horizontally oriented ice lenses, both in the expanded sample above the soil box and in the tailings contained in the soil box.

Figure 5-11 | Color change observed for MI/MN blend tailings due to sublimation in freezer and wind tunnel: a) samples prior to freezing, b) samples after 7 days of freezing, c) sample prior to wind tunnel testing, and d) sample after wind tunnel testing for 30 seconds at $97.7 \mathrm{~km} / \mathrm{hr}$.

Figure 5-12 | Qualitative data supporting sublimation and dust generation for frozen $\mathrm{MI} / \mathrm{MN}$ blend tailings: a) and b) light surface layer underlain by darker layer clearly shows depth of sublimation, and c) and d) surface ice lenses are empty, indicating that ice has sublimated. 202

Figure 5-13 | Wind Tunnel Data for $16 \mathrm{MI} / \mathrm{MN}$ blend tailings samples, showing mass lost due to wind erosion (30 seconds at $96.6 \mathrm{~km} / \mathrm{hr}$ ) varying with sample vertical expansion $\left(\mathrm{R}^{2}=0.86\right)$.

Figure 5-14 | Ball Drop Data for $18 \mathrm{MI} / \mathrm{MN}$ blend tailings samples, showing average ball

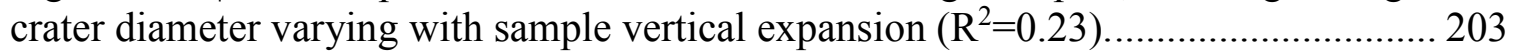

Figure 6-1 | BSCs growing on MI-mag (left) and MI-hem (right) tailings surface........ 219

Figure 6-2 | Eagle River crust growing on MI-hem tailings (left) and viewed under light microscope at 40x magnification (right). It should be noted that the MI-hem tailings are naturally red, and the crust is black, showing almost total coverage of tailings.

Figure 6-3 | Schematic of bioreactor inoculation, where one batch of $400 \mathrm{~mL}$ inoculum was divided evenly between 6 soil boxes, featuring removable collars to provide extra 
headspace in which the inoculum could reside until percolating through the tailings sample.

Figure 6-4 | Eagle River inoculum viewed under light microscope at 40x magnification, showing the presence of both filaments and unicellular organisms. 232

Figure 6-5 | Digital photographs of ER1 bioreactors used to qualitatively document crust development (a) at the start of treatment (day 3) with a light green color completely covering the tailings surface, (b) after full crust development (day 37) with a darker green or black color completely covering the tailings surface, and (c) at the end of treatment (day 134) with a thick and healthy black crust. Circular imprints in the crust are from the spectroradiometer probe.

Figure 6-6 | Digital photographs of ER3 bioreactors used to qualitatively document crust development (a) at the start of treatment (day 1) with little to no coverage of the tailings surface, (b) at the end of treatment (day 42) for a healthy bioreactor with a darker green crust completely covering the tailings surface, and (c) at the end of treatment (day 42) for an unhealthy bioreactor with patchy crust coverage of the tailings surface. 234

Figure 6-7 | Digital photographs of ER4 bioreactors used to qualitatively document crust development (a) at the start of treatment (day 1) with little to no coverage of the tailings surface, (b) after reinoculation (day 24) with a more noticeable green color completely covering the tailings surface, and (c) at the end of treatment (day 31) with a thick and healthy dark crust.

Figure 6-8 | NDVI used for quantitative crust monitoring of (a) Eagle River 1 bioreactors, (b) Eagle River 3 bioreactors, (c) Eagle River 4 bioreactors, and (d) BG-11 control bioreactors, showing that crust development can be monitored using NDVI, full crust coverage can be identified when NDVI levels off, and crust health can be assessed by watching for a decrease in NDVI.

Figure 6-9 | Biomass characterization data for Eagle River bioreactors, showing that biomass concentration is a function of treatment time and that biomass of treated samples far exceeds biomass of BG-11 controls. Each point represents the average value for each bioreactor, and the error bars represent standard deviation. 239

Figure 6-10 | Relationship between NDVI and biomass concentration, showing that NDVI has a stronger correlation with chl a $\left(\mathrm{R}^{2}=0.85\right)$ than it does with EPS $\left(\mathrm{R}^{2}=0.50\right)$. Each point represents the average final NDVI for each bioreactor set versus the average biomass concentration for each bioreactor, and the error bars represent standard deviation. 240

Figure 6-11 | Scanning electron microscopy of (a) untreated magnetite tailings, and (b-f) Eagle River crust on MI/MN blend tailings. (a) The untreated tailings particles are angular and do not feature any notable coating. (b) A visible coating, presumed to be EPS, can be observed on the surface of the tailings, as well as some rod-shaped organisms and filaments. (c) The same visible EPS coating can be seen, as well as some rod-shaped organisms and multiple, intertwined filaments; the connection of a filament to the crust/tailings surface, filament branching, and particle attachment to the filament are 
highlighted in the rectangle and can be seen in more detail in (d). (e) Filament connections to the crust/tailings surface and intertwined filaments are shown. (f) Zooming in shows additional detail, including pores in the filaments and crust..

Figure 6-12 | Eagle River bioreactors (a) before freezing, (b) after freezing, and (c-d) after removal from freezer. (a) and (b) show that freezing and sublimation does not cause damage to the crust surface; the back right sample pictured in (b) was covered by underlying tailings during sample saturation, but crust is intact underneath thin tailings layer. (c) and (d) show that the underlying tailings mimic freezing behavior, ice lens formation, and sublimation (with the depth of sublimation clearly defined by the tailings color change in (d)) of untreated tailings.

Figure 6-13 | Wind tunnel results for untreated MI/MN blend tailings (Zwissler et al. in preparation) and for BG-11 control bioreactors (this work). The trends of the BG-11 control bioreactors agree with the trends observed for the untreated MI/MN blend tailings (dashed line, $\mathrm{R}^{2}=0.86$ ), indicating that the datasets can be combined to establish baseline conditions (solid line, $\mathrm{R}^{2}=0.77$ ). Each point represents results for a single sample....... 246

Figure 6-14 | Wind tunnel results for ER bioreactors and compared to the baseline conditions of untreated MI/MN blend tailings (Zwissler et al. in preparation) and for BG11 control bioreactors. All ER bioreactors with mass lost falling below the trendline are interpreted to resist wind erosion better than untreated tailings. All ER bioreactors with mass lost above the trendline failed due to conditions unrepresentative of field conditions. Each point represents results for a single sample.

Figure 6-15 | Baseline ball drop results for untreated MI/MN blend tailings (Zwissler et al. in preparation) and for BG-11 control bioreactors. The trends of the BG-11 control bioreactors agree with the trends observed for the untreated MI/MN blend tailings (dashed line, $\mathrm{R}^{2}=0.23$ ), indicating that the datasets can be combined to establish baseline conditions (solid line, $\mathrm{R}^{2}=0.21$ ). Each point represents the average diameter of 5 craters, and error bars represent standard deviation.

Figure 6-16 | Ball drop results for ER bioreactors compared to untreated MI/MN blend tailings (Zwissler et al. in preparation) and BG-11 control bioreactors. All bioreactors fall below the untreated trendline, indicating that the presence of a crust helps to resist impact. All bioreactors with a crater diameter of zero had a crust that absorbed the impact and rebounded without leaving behind a crater. Each point represents the average diameter of 5 craters, and error bars represent standard deviation. 250 


\section{List of Tables}

Table 2-1 | Geotechnical characterization of mine tailings samples from MI-mag, MI-

hem, and $M N-m a g$ impoundments.

Table 2-2 | Details of thermal remote sensing laboratory testing conducted per sampling event.

Table 2-3 | Description of each FLIR object parameter and how the parameter was quantified.

Table 2-4 | Details of site visits to MI-mag, MI-hem, and MN-mag impoundments. ...... 83

Table 2-5 | Details of field testing in Oct 2014 for geotechnical characterization and ground truthing to validate satellite data. Seven sites around the northern half of the MNMag impoundment were studied

Table 2-6 | Details of field testing in May 2015 for geotechnical characterization and ground truthing to validate satellite data. Thirty sites around the south-eastern region of the MN-Mag impoundment were studied.

Table 2-7 | Details of field testing in June 2016 for geotechnical characterization and ground truthing to validate satellite data. Fifteen sites on the eastern side of the MI-Mag impoundment were studied, and measurements were taken pre- and post-heating due to a 6 hour diurnal heating cycle 85

Table 2-8 | Comparison between satellite and UAV thermal remote sensing .................. 88

Table 2-9 | Description of measurements made during sample freezing/sublimation for MI-mag and MN-mag tailings.

Table 2-10 | Description of measurements made during wind tunnel testing for MI-mag and $\mathrm{MN}$-mag tailings.

Table 2-11 | Summary of inoculum for ER1, ER3, and ER4 bioreactors, including absorbance used to compare inoculum biomass density.

Table 3-1 | Details of thermal remote sensing laboratory testing conducted per sampling event.

Table 3-2 | Description of each FLIR object parameter and how the parameter was quantified.

Table 3-3 | Statistical information for each variable considered in relationship between moisture content and thermal remote sensing for MI-mag tailings, $n=45$.

Table 3-4 | Statistical information for each variable considered in relationship between moisture content and thermal remote sensing for MN-mag tailings, $\mathrm{n}=48$. 139

Table 3-5 | Variable assessment statistics for moisture content multivariate linear regression developed for MI-mag tailings. 
Table 3-6 | Variable assessment statistics for moisture content multivariate linear regression developed for $\mathrm{MN}$-mag tailings.

Table 4-1 | Details of field testing in June 2016 for fifteen sites on the eastern side of the MI-Mag tailings impoundment, where measurements were taken pre- and post-heating due to a 7 hour diurnal heating cycle. .

Table 4-2 | Description of each FLIR object parameter and how the parameter was quantified.

Table 5-1 | Description of measurements made during sample freezing and sublimation for $\mathrm{MI} / \mathrm{MN}$ blend tailings.

Table 5-2 | Description of measurements made during wind tunnel testing for MI-mag and MN-mag tailings.

Table 6-1 | Summary of inoculum for ER1, ER3, and ER4 bioreactors, including absorbance used to compare inoculum biomass density.....

Table 6-2 | Summary of biomass characterization data for Eagle River Bioreactors and

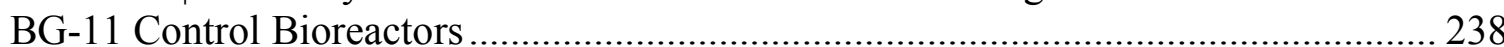




\section{Preface}

The material presented in Chapters 3-6 has been submitted for review or is in preparation to be submitted for publication.

Chapter 3, "Thermal remote sensing for moisture content of mine tailings: laboratory study" is a manuscript that was submitted to Environmental and Engineering Geoscience. The contributions made by each co-author are as follows:

- Bonnie Zwissler: majority of data collection, analysis, and writing.

- Thomas Oommen: assistance with data analysis, proofreading.

- Stan Vitton: technical assistance, proofreading.

- Eric Seagren: technical assistance, proofreading.

Chapter 4, "Thermal remote sensing for moisture content of mine tailings: field study" is a manuscript that is in preparation for submission. The contributions made by each coauthor are as follows:

- Bonnie Zwissler: majority of data collection, analysis, and writing.

- Thomas Oommen: assistance with data analysis, proofreading.

- Stan Vitton: assistance with data collection, proofreading.

- Eric Seagren: technical assistance, proofreading.

Chapter 5, "Laboratory method to generate dust via sublimation to study cold weather dusting phenomenon for fine-grained sediments" is a manuscript that is in preparation for submission. The contributions made by each co-author are as follows:

- Bonnie Zwissler: majority of data collection, analysis, and writing.

- Stan Vitton: assistance with methods and data analysis, proofreading.

- Eric Seagren: technical assistance, proofreading.

- Thomas Oommen: technical assistance, proofreading.

Chapter 6, "Biological Soil Crust monitoring and laboratory testing to explore dust suppression potential for mine tailings" is a manuscript that is in preparation for submission. The contributions made by each co-author are as follows:

- Bonnie Zwissler: majority of data collection, analysis, and writing.

- Eric Seagren: assistance with methods, data analysis, and proofreading.

- Thomas Oommen: technical assistance, proofreading.

- Stan Vitton: technical assistance, proofreading. 



\section{Acknowledgements}

This research would not have been possible without the financial, technical, and moral support of many people and organizations. Many thanks to all who provided such support, especially those listed below.

This material is based upon work supported by the National Science Foundation under Grant No. 1234126, with additional support from the Michigan Space Grant Consortium, the Michigan Technological University (MTU) Department of Civil and Environmental Engineering (through TA support), and the MTU Graduate School (through a Dean's Fellowship and a Doctoral Finishing Fellowship).

The cooperation of Cliffs Natural Resources, Inc. of Cleveland $\mathrm{OH}$ was essential to the success of this project. Specifically, Kris Scherer, Perry Fiorini, and Dean Korri provided technical guidance, allowed access to their mine tailings impoundments in both northern Michigan and Minnesota, and provided field support.

The guidance of co-advisors Dr. Thomas Oommen and Dr. Eric Seagren, as well as committee members Dr. Stan Vitton and Dr. Ann Maclean, was essential to the success of this project. They provided countless hours of guidance (and entertainment) in meetings, and their input was crucial to all stages of the project, most notably the (frequent and unrelenting) troubleshooting required to successfully complete all parts of the project. The author will always attribute a large deal of her technical and professional development to the skills learned from her advisors and committee during this project.

The assistance of the large team of graduate student and undergraduate student assistants was also essential to be able to complete the laboratory and field testing to support such a large and diverse project. Noah Buikema, who started on the project as an undergraduate assistant and continued as a MS student, was an enormous asset to the project, and Noah's presence as a colleague and office mate was under-appreciated by the author for much of his time at MTU. Other undergraduate assistants who contributed to the project via data collection, management, and analysis include, in order of introduction 
to the project: Daniel Cerminaro, Christa Meingast, Kevin Madson, Becca Green, Maggie Ziols, Rebekka Guyon, Harrison Zost, Abby Meisel, and Juli Mickle.

Beyond the project team, there were a large number of people at MTU who provided assistance. Rob Fritz and Kiko de Melo e Silva provided a great deal of laboratory technical support and fabrication assistance for all parts of the project. Dr. Jeffrey Allen allowed the relocation and modification of the wind tunnel, without which the project would have been much more difficult. The US Forest Service's Northern Research Station located in Houghton, MI allowed the use of their spectroradiometer and plant probe, which proved to be useful for monitoring of biological soil crusts. Dr. Janice Glime provided assistance in the visual assessment of the biological soil crust organisms. And Ron Frederick provided frequent and rapid assistance with HVAC issues in the freezing/sublimation laboratory apparatus used.

Lastly, this research would not have been possible without the support of family and friends, near and far. Whether the support was offered in the form of motivational words, a listening ear for problem solving and (seemingly endless) complaining, care packages, phone calls, outdoor adventures as a form of distraction, or "broken arm support services", it was much appreciated. 


\begin{abstract}
Mining operations produce massive volumes of mine tailings, which are deposited as slurry into permanent tailings impoundments. An important and heavily regulated environmental hazard associated with mine tailings impoundments is fugitive dust emissions. Wind erosion of mine tailings impoundments and the resulting dusting events, especially those caused by cold weather dusting, remain an on-going challenge for the mining industry. The overall goal of this research was to develop and evaluate effective, economical, and sustainable solutions to two major issues facing mine tailings impoundments with regards to dusting: (1) dust monitoring/detection and (2) dust reduction/prevention. Specifically, the research studied iron mine tailings and used (1) laboratory and field testing to assess the utility of thermal remote sensing techniques for dust monitoring, and (2) laboratory testing to assess the ability of biological soil crusts to reduce dust emissions due to cold weather dusting. A laboratory model was developed to use thermal remote sensing and other atmospheric variables to predict surface moisture content and strength of iron mine tailings. Though this relationship was not found to be directly applicable to field remote sensing, this research suggests that a model could be developed using field data to predict surface moisture content using thermal remote sensing, which would be a useful tool for tailings impoundment managers to employ for dust emissions detection. Additionally, a method was developed and validated that exposed laboratory tailings sample to freezing and sublimation conditions that are representative of those experienced at tailings impoundments located in cold-weather climates, and the dust emissions and strength of these tailings samples was characterized using a wind tunnel and ball drop testing. Lastly, biological soil crusts originating from locally-sourced organisms were grown on laboratory tailings samples, and when exposed to freezing/sublimation and tested with wind tunnel and ball drop testing, the biological soil crusts were found to provide resistance to wind erosion and increased surface strength. These results are important, both for the understanding of dust emissions and potential dust mitigation treatments for tailings impoundments, and also for broader issues of wind erosion and dust emissions of soil both globally and on other planets.
\end{abstract}





\section{Chapter 1 Introduction, Objectives, and Literature Review}

\subsection{Introduction}

Massive volumes of waste materials are produced each year by mining operations. According to the U.S. Environmental Protection Agency (USEPA), between one and two billion tons of mine waste are produced annually in the U.S., excluding coal (USEPA 2014). Mine tailings result from crushing rock for ore beneficiation, which yields finely crushed rock particles (average particle size is $20 \mu \mathrm{m}$, with a significant portion between 1 and $10 \mu \mathrm{m})$. Once produced, the tailings are often deposited in slurry form into permanent tailings impoundments (Vick 1983). Over 3500 active tailings impoundments exist worldwide (Davies et al. 2000). The impoundments constructed to contain tailings are among some of the largest earthen structures in the world, and are subject to intense regulatory and public attention because of the land areas they disturb and the hazards associated with the mining waste they contain (Vick 1983). One of the many important environmental hazards associated with tailings impoundments is air pollution from blowing dust (Buck and Gerard 2001).

Blowing dust due to wind erosion, including wind erosion of mine tailings, can negatively impact human activity because it can cause poor visibility for drivers and can cause respiratory health problems (Bang et al. 2009). Blowing dust is regulated by the USEPA as airborne particulate matter based on particle size and particle concentration. Particles of concern include coarse particles (diameters 2.5-10 $\mu \mathrm{m}$ ) and fine particles (diameters less than $2.5 \mu \mathrm{m}$ ). The latter are of particular concern because they are small enough to reach the lower portions of the human respiratory tract and can cause respiratory health problems including damage to lung tissue, cancer, and premature death (USEPA 2012). Under the Clean Air Act, the current national air quality standard limits particles smaller than $2.5 \mu \mathrm{m}$ to $12 \mu \mathrm{g} / \mathrm{m}^{3}$ for an annual mean, or $35 \mu \mathrm{g} / \mathrm{m}^{3}$ for a 24 -hour concentration, and limits particles $2.5-10 \mu \mathrm{m}$ to $150 \mu \mathrm{g} / \mathrm{m}^{3}$ for a 24-hour concentration (USEPA 2014). Dusting events at tailings impoundments can lead to violations of these regulations. For example, according to the Michigan Department of Environmental Quality, on November 15, 1996, the Empire Mine (located in Palmer, MI, and operated 
by Cliffs Natural Resources) had a dusting event that deposited airborne tailings particulates up to ten miles from the impoundment, caused respiratory health issues to the local residents, and interrupted transportation due to poor visibility.

It is crucial to understand how to mitigate dusting from tailing due to wind erosions and how to detect when the tailings may be susceptible to dusting. Dust emissions occur when the wind's shear stress on the tailings surface exceeds the tailings' shear strength (friction and cohesion) holding them in place (Greeley and Iversen 1987; Nickling and McKenna Neuman 2009), and the amount of dust produced is a function of the moisture of the soil, the grain size and the weight of the individual particles, the speed of the wind, and the roughness of the soil surface (Marticorena and Bergametti 1995). Therefore, any attempt to mitigate dusting must either increase the surface strength of the tailings or decrease the force of the wind acting on the tailings, while accounting for the spatial and temporal variations in moisture, grain size, and soil roughness. Some traditional dust mitigation solutions for mitigating dusting include water inundation, mulching cover, crusting or binding agents, and wind fences. The issue of dust emissions is complex enough when considering warm climates; but an additional complicating factor, called cold weather dusting, is the more complex and a more significant contribution to dust emissions at mine tailings impoundments located in cold climates. Cold weather dusting is driven by the processes of freeze-thaw and sublimation, which leave behind a surface layer of loose, dry, and unstable tailings that require much lower wind speeds to become airborne and generate dust.

Whether considering warm- or cold-weather dusting, understanding how the shear strength and other soil surface properties change spatially and temporally is complex, so monitoring the tailings to determine if and when certain regions of the impoundment may be susceptible to dusting is important. The traditional approach for monitoring dust emissions from tailings involves collecting dust samples from gauges, which provides limited spatial coverage of the tailings and is also costly, labor intensive, and affected by trafficability. Because of these limitations, the problem may not be detected before the dusting event occurs. 
Remote sensing may be an alternative method to monitor the susceptibility of tailings impoundments to dusting because it provides a synoptic view of surficial processes with high spatial and temporal resolutions, making it useful for monitoring and detecting change. To utilize the high spatial and temporal resolution of remote sensing for monitoring the susceptibility of tailings impoundments to dusting, it will be critical to be able to derive the strength and the moisture content in the top layer of the tailings using remote sensing. Thermal remote sensing (particularly thermal inertia) has been shown to have a linear relationship with moisture content (Liu and Zhao 2006). In addition, thermal remote sensing is a typical method used to indirectly determine relative soil moisture (Minacapilli et al. 2012). Therefore, it is hypothesized that if a relationship between the surface strength/dusting susceptibility and moisture content of the magnetite tailings from the Empire Mine can be developed, then thermal remote sensing (particularly thermal inertia) data can be indirectly related to the strength/dusting susceptibility of the tailings.

Once the regions of a tailings impoundment that are susceptible to dusting are identified, dust mitigation solutions can be applied. Any attempts to mitigate dusting on tailings impoundments must either increase the surface shear strength of the tailings or decrease the force of the wind acting on the tailings. Most mitigation techniques focus on increasing the strength of the tailings, because the speed and direction of the wind are so variable and are for the most part out of human control. There are many mitigation techniques that are currently used at tailings impoundments to attempt to increase the surface strength of the tailings, and they rely on chemical or physical additives (Bang et al. 2009), including water inundation, sludge cover, mulching cover, and crusting or binding agents. However, many of these physical and chemical treatments have environmental consequences, have high costs for application, and may lose effectiveness with time due to factors like UV degradation (Bang et al. 2009). Therefore, a more effective, economical, and longer lasting solution without environmental consequences is desirable. 
Several research groups are investigating the use of microbial soil stabilization as an alternative to chemical and physical treatments to increase surface strength and resist soil erosion. The most common process uses a bacterium, Sporosarcina pasteurii, which hydrolyzes urea, creating an environment conducive to the precipitation of calcium carbonate. This process is commonly called microbially induced calcite precipitation (MICP) (Bang and Bang 2011; Bang et al. 2009; Buikema et al. accepted; Chu et al. 2012; DeJong et al. 2006; Stocks-Fischer et al. 1999; Whiffin et al. 2007). In most soils, MICP requires the addition of urea and calcium. The majority of research concerning the use of MICP for surficial strength increase has been tested on sand samples at laboratory scales, and no research has been published MICP for mine tailings. Laboratory testing with sandy soils has shown an increase in surface strength and reduced wind erosion susceptibility (Bang and Bang 2011; Bang et al. 2009), but scaling MICP to field applications would have to address issues such as cost, growth time, uniformity of bacteria/nutrient application, and longevity (DeJong et al. 2013).

As stated above, these physical, chemical, and microbial treatments have demonstrated varying degrees of success in helping soil to resist wind erosion by increasing surface strength. Rather than developing new treatments, an alternative approach is to take inspiration from one of nature's most successful solutions to erosion resistance, biological soil crusts. Biological soil crusts are naturally occurring and cover otherwise bare soil, providing an increased surface strength and resistance to soil erosion. Biological soil crusts have been proven in natural systems to reduce wind and water erosion (Eldridge and Greene 1994). Soil featuring biological crusts that are welldeveloped has shown 2 to 130 times more resistance to soil erosion than bare soil or soil with crusts that are not fully developed (Belnap and Gillette 1997; Belnap et al. 2001; Leys and Eldridge 1998; McKenna Neuman et al. 1996; Williams et al. 1995). If reductions in soil erosion of this magnitude have been documented for natural systems, it indicates that it may be possible for similar results to be obtained from human stimulation of biological soil crusts in other locations that are susceptible to erosion. The growth of biological soil crusts on previously un-crusted soil has been demonstrated successfully in the laboratory (Liu et al. 2008; McKenna Neuman and Maxwell 1999; McKenna Neuman 
and Maxwell 2002; McKenna Neuman et al. 2005; McKenna Neuman et al. 1996; O'Brien and McKenna Neuman 2012). Biological soil crusts have, and will continue to, colonize and grow on previously bare soil in natural settings unaided by human activity. The uncertainty lies in the human intervention component, specifically if biological soil crusts can be cultivated on tailings impoundments and used to suppress dusting. It is hypothesized that if biological soil crusts can be promoted on the surface of mine tailings impoundments, the surface strength will be increased, and the susceptibility to dusting decreased.

In summary, mine tailings are susceptible to wind erosion and dusting, and the resulting fugitive dust emissions can be harmful to environmental and human health. Being able to detect when mine tailings may be susceptible to dusting and increase the erosion resistance of surface tailings are two critical issues that continue to pose challenges to the mining industry; both of these issues must be addressed if fugitive dust emissions are to be managed. Therefore, this research addressed two main questions: (1) Can remote sensing be used to detect the dust susceptibility of mine tailings (when/where dusting will occur)? and (2) Can biological soil crusts be used to decrease the dusting susceptibility of mine tailings (reduce/eliminate dusting)?

\subsection{Scope of Study and Objectives}

Wind erosion of mine tailings impoundments and the resulting dusting events, especially those caused by cold weather dusting, remain an on-going challenge for the mining industry. The overall goal of this research was to develop and evaluate effective, economical, and sustainable solutions to two major issues facing mine tailings impoundments with regards to dusting: dust monitoring/detection and dust reduction/prevention. Specifically, the research used thermal remote sensing techniques to address dust monitoring and detection, and used biological soil crust cultivation to address dust reduction or prevention. The overall hypotheses driving the proposed research were: 
1. Thermal remote sensing can be used to detect variations in moisture content and surface strength for mine tailings at both laboratory and field scales.

2. Biological soil crusts can be cultivated on mine tailings and can be used to increase the strength of the tailings' surface, and decrease the dust emissions due to cold-weather dusting from mine tailings at laboratory scales.

These hypotheses were investigated through a combination of laboratory and field experimentation. The specific objectives of the proposed research were to:

1. Objective 1: Develop a laboratory relationship between thermal remote sensing and moisture content/surface strength for untreated mine tailings.

2. Objective 2: Apply laboratory relationship from objective 1 to field scale using remote sensing coupled with ground truthing, to generate spatial/temporal maps of variation in surface moisture and strength.

3. Objective 3: Develop laboratory methods to generate and quantify dust caused by cold weather dusting (sublimation) for untreated tailings.

4. Objective 4: Assess if biological soil crusts are a viable dust suppressant/treatment for tailings that experience cold-weather dusting via biomass characterization and wind tunnel testing, to determine if crusts increase surface strength and decrease dust susceptibility.

Completion of these objectives has led to a greater understanding of the problem of wind erosion at mine tailings impoundments, improved tools for monitoring the susceptibility to dusting at tailings impoundments, and innovative approaches for preventing wind erosion and dusting at tailings impoundments. Importantly, the results of this study may be more broadly applicable to the global problem of soil erosion.

The following chapters detail the research conducted to test the project hypotheses and satisfy the project objectives. The remainder of Chapter 1 reviews literature relevant to soil erosion, mine tailings, remote sensing, biological soil crusts, and surficial strength/erosion susceptibility testing. Chapter 2 outlines the detailed laboratory methods 
that were used to address each objective. Chapter 3 is a peer-reviewed publication (in review) that describes the laboratory methods to simulate cold weather, sublimationbased dusting for iron tailings. Chapter 4 is a draft publication (in preparation) that describes the laboratory-based study of using thermal remote sensing to monitor for moisture content and surface strength of iron tailings. Chapter 5 is a draft publication (in preparation) that describes the application of the laboratory relationship between thermal remote sensing and moisture content/strength to field scale remote sensing. Chapter 6 is a draft publication (in preparation) that describes using biological soil crusts to reduce dust emissions for laboratory samples of iron tailings. And lastly, Chapter 7 is a synthesis of the research and discusses broader impacts.

\subsection{Literature Review}

\subsubsection{Soil Erosion}

Soil strength can be defined as a soil's ability to withstand an applied load without failure (Duncan and Wright 2005). In many instances, loading and failure of soil is considered in the context of assessing new or existing structures or slopes. However, soil strength can also be considered in the context of soil erosion. The most common erosion mechanisms that cause soil erosion are wind and water in the form of precipitation (Lal 1994). Soil erosion is a concern because it transports sediment from where it is located and often desired - like agricultural fields - to locations where it is not desired-like roadways in the case of wind erosion (Barnes and Connor 2014; Huszar and Piper 1986), or riverbeds and drainage ditches in the case of water erosion (Morgan 2009). The focus of this work is wind erosion. In the case of wind erosion, dust emissions can more directly impact human life by causing poor visibility and road conditions for drivers and causing respiratory health problems (Bang et al. 2009), including damage to lung tissue, cancer, and premature death (USEPA 2012).

To understand the erosion mechanisms, one must understand how the soil resists or is affected by wind. Studies analyzing the susceptibility of sediments to dust emissions have concluded that the dust emissions do not occur until the shear stress exerted by the wind 
on the sediment surface exceeds the shear strength (frictional and cohesive forces) that holds the sediments in place (Greeley and Iversen 1987; Nickling and Neuman 2009); the amount of dust produced is a function of the moisture of the soil, the grain size and the weight of the individual particles, the speed of the wind, and the roughness of the soil surface (Marticorena and Bergametti 1995). Soil moisture affects dust generation because the presence of water provides a cohesive force to hold the soil particles in place, and when the soil is submerged under water, there is no way for wind to generate dust. The effects of grain size and particle weight on dust production are self-explanatory, as a higher wind speed would be required to transport larger and heavier particles. Soil roughness affects dust generation because a smooth surface encourages laminar wind flow while a rough surface encourages turbulent flow or even can cause a turbulent boundary layer to form, which affects the direction and speed of the wind interacting with the soil surface. When the wind is able to overcome these factors and erode soil, the resulting dust produced can also be considered airborne particulate matter.

The USEPA regulates airborne particulate matter based on particle size and particle concentration. Under the Clean Air Act, in 1987, the USEPA established the PM-10 standard (USEPA 2012), which limited the concentration of airborne particles smaller than 10 micrometers in diameter. Particles of this size are a concern to public health, as they are small enough to reach the lower portions of the respiratory tract. PM-10 set the national air quality standard for airborne particles smaller than 10 micrometers to 50 $\mu \mathrm{g} / \mathrm{m}^{3}$ for an annual mean or $150 \mu \mathrm{g} / \mathrm{m}^{3}$ for a 24-hour concentration. In 2012, the PM-10 standard was replaced with the PM-2.5 standard (USEPA 2014), because research showed that particles less than 2.5 micrometers in diameter were the cause of the most serious adverse health effects. PM-2.5 set the national air quality standard for airborne particles smaller than 2.5 micrometers to $12 \mu \mathrm{g} / \mathrm{m}^{3}$ for an annual mean or $35 \mu \mathrm{g} / \mathrm{m}^{3}$ for a 24-hour concentration, and retained the PM-10 24-hour concentration of $150 \mu \mathrm{g} / \mathrm{m}^{3}$ for particles 2.5 to 10 micrometers. Mines in the United States are required to follow the Clean Air Act and other applicable regulations (e.g. National Emission Standards for Hazard Air Pollutants (NESHAP) for Taconite Iron Ore Processing). In addition, mines must hold permits like Renewable Operating Permits and develop/follow fugitive dust 
emissions control plans (Scherer and Ketzenberger 2014), because of the potential for wind erosion to produce airborne particulate matter from mine tailings.

\subsubsection{Mine Tailings}

Mine tailings impoundments must safely contain large volumes of tailings, and can be made from existing open pits or natural depressions, or they can be constructed using embankments made with local materials, often including tailings (USEPA 1994). Tailings can be deposited into impoundments as slurry (Vick 1983), paste (Theriault et al. 2003), or dry-stacked (Davies and Rice 2001). Tailings impoundments are subject to intense regulatory and public attention because of the land areas they disturb and the hazards associated with the mining waste they contain (Vick 1983). These hazards can include: catastrophic dike failure, which can flood adjacent land and waterways with tailings; soil contamination by heavy metals, which can limit soil fertility or sterilize it completely; water contamination from heavy metals and/or acid mine drainage; and air pollution from dust emissions (Buck and Gerard 2001; Hoffert 1947). Some of these hazards can be prevented with a thorough engineering design, but others, like dust emissions, are often handled after tailings are already deposited through mitigation (short-term) or reclamation (long-term) activities. This work focuses on the hazard of fugitive dust emissions from mine tailings impoundments.

As mentioned previously, dust emissions occur when the shear stress exerted by the wind on the tailings surface exceeds the shear strength that holds the tailings in place. For tailings impoundments located in cold-weather climates, wind strength can exceed tailings strength due to two main phenomena: warm weather dusting and cold weather dusting.

Warm weather dusting relies on the conventionally understood processes of wind erosion of soil. Tailings are commonly deposited into the impoundment as a slurry, typically composed of $15-55 \%$ solids by mass (Vick 1983). As they are deposited, the tailings segregate by particle size; larger particles are found closer to the discharge point, and finer particles travel farther from the discharge point (Price 1998; Vasher 1999). 
With time and exposure to the atmosphere, the tailings dry and can be degraded by wind, rain, UV exposure, and other factors. At the point that the wind stress exceeds shear strength of the surface tailings, dusting occurs. Warm weather dusting occurs in warmand cold-weather climates, though it is not the main concern for tailings impoundments in cold-weather climates.

Cold weather dusting is a more complex phenomenon, and a more significant contributor to dust emissions at mine tailings impoundments located in cold climates. Cold weather dusting is driven by the processes of freeze-thaw and sublimation.

Freeze-thaw occurs when soil loses enough heat for the pore water to freeze. This freezing occurs from the ground surface down to a depth that is controlled by the magnitude of soil heat loss, with the soil heat loss depending on the thermal properties of the soil (Gatto 1995). Freezing of the pore water leads to expansion, as water expands about nine percent by volume when it is frozen to ice. Ice lenses can form if the freezing front propagates slowly enough, and the capillarity is sufficiently high for water to be transported to the surface (Gatto 1995). Silts are the soils that are the most susceptible to these freeze-thaw phenomena, because they have high capillarity, which pulls water to the freezing front, and a sufficiently high hydraulic conductivity to allow water to flow through (Gatto 1995; Michalowski and Zhu 2006). In comparison, the freezing behavior of soil with varying particle-size distribution and mineralogy also varies, though particlesize distribution is what tends to control soil freezing behavior. For those soils that experience freeze-thaw, the destabilizing effects develop during the freezing phase. If/when thawing then occurs, larger voids are present in the soil matrix and the soil structure is disturbed due to the ice expansion during freezing. After thawing, there is also a higher moisture content present at the surface. These factors combined lead to a reduction in shear strength. For example, there can be as much as a 50 percent reduction in soil shear strength after a single freeze-thaw cycle (Gatto 1995).

If, however, the pore ice and ice lenses do not thaw but instead sublimate, the instability is increased dramatically. Sublimation is the process by which water transitions directly from the solid phase (ice) to the gaseous phase (water vapor) without 
passing through the liquid phase. When sublimation of the pore ice and ice lenses occurs, the tailings desiccate. As a result, the larger voids become filled with air, which provides no support to the soil matrix, significantly weakening it. There is also no apparent cohesion from water suction holding the tailings particles together, so the wind only has to overcome the frictional component of shear strength to cause the particles to become airborne. The depth of sublimation determines the amount of available sediment for dusting (Huang and Aughenbaugh 1987), but the wind speeds required for fugitive dust emissions due to cold weather dusting are significantly lower than those required for warm weather dusting. Sublimation at tailings impoundments located in cold regions is most severe in late fall and early spring, when there is no snow cover and sub-freezing temperatures are achieved. The rate and depth of sublimation can be affected by several factors, including the: ambient temperature, with the most significant rate of sublimation occuring right at the freezing point $\left(0^{\circ} \mathrm{C}\right)$; relative atmospheric humidity, with a decrease in relative humidity leading to an increase in sublimation; wind speed, with an increase in wind speed leading to an increase in sublimation; and soil moisture content, with tailings having higher moisture contents experiencing more sublimation because of the increased void space and the low likelihood that dust control measures have been taken due to trafficability concerns (Huang and Aughenbaugh 1987; Vasher 1999).

Each tailings impoundment is unique and varies in impoundment design/construction, footprint and volume of tailings contained, duration of operation, physical/chemical/mineralogical composition of tailings, surface water and groundwater regime, climate, and seismicity (Vick 1983), among other factors. Thus, a study of a mine tailings impoundment must consider the site-specific conditions.

In Michigan's Upper Peninsula alone, there are multiple active tailings impoundments. Due to the duration of their mining operations and their close proximity to Michigan Technological University, the tailings from two iron mines - the Empire tailings impoundment and the Gribben tailings impoundment (waste from the Tilden Mine) — have been studied with both in-situ and laboratory testing. It should be noted that laboratory testing of tailings is almost always conducted on reconstituted samples, as the 
tailings are easily liquefiable due to their high moisture content and relatively low density, and samples tend to liquefy when transported from the impoundment to the laboratory. Warm weather dusting has been documented on both impoundments (Vasher 1999), though it is not the main concern. Cold weather dusting is the more complex and more significant contribution to dust emissions at the Empire and Gribben impoundments.

Multiple studies have been conducted on the Empire tailings by researchers at Michigan Tech. Arnoldt (1970) conducted laboratory testing, including direct- and triaxial-shear tests, to study the pore water pressure development in the Empire tailings. Pawloski (1979) studied the stability of a trial embankment constructed with tailings through field testing, laboratory testing, and slope stability modelling. King (1981) observed the stability of that same trial embankment after construction using instrumentation, including piezometers and extensometers. Newton (1981) provided a field characterization of the Empire tailings impoundment, and also studied the onedimensional compressibility of the tailings with laboratory consolidation testing. Wheeler (1991) performed a laboratory study to characterize the stress-strain behavior of the normally consolidated tailings under undrained shear through triaxial testing. Price (1998) performed a field characterization of the Empire tailings and also studied the effectiveness of possible dust suppressants through laboratory testing. Vasher (1999) performed field testing to study fugitive dust emissions from the tailings impoundment using a portable wind tunnel. Finally, Muszynski (2000) studied the variation in void ratio due to aging for coarse tailings using triaxial testing coupled with sample wax impregnation and imaging. These studies provide a general understanding of the conditions to be expected in the Empire tailings impoundment, as well as the stress response of the tailings.

Multiple studies have also been conducted by Michigan Tech researchers on the tailings from the Tilden mine deposited in the Gribben basin. Grannemann (1984) modelled the hydrogeology of the Tilden tailings impoundment. Grosser (1996) developed a method to reconstitute and saturate normally consolidated laboratory 
samples for triaxial testing without disturbing the soil structure through backpressure saturation. Zarling (1997) studied the undrained and drained shear behavior of normally consolidated tailings through triaxial testing in the laboratory. Priest (1997) studied the compressibility of the tailings through laboratory consolidation testing. Price (1998) performed a field characterization of the Gribben tailings and also studied the effectiveness of possible dust suppressants through laboratory testing. Finally, Vasher (1999) performed additional field characterization of the Gribben tailings. Vasher (1999) also indicated that the Gribben basin had lower access due to trafficability and that the hematite tailings from this basin were more difficult to work with in the laboratory; this might indicate why more studies focused on the Empire tailings rather than those from Gribben and why most studies featured laboratory tests rather than field testing.

There are many more active and inactive tailings impoundments in the region, country, and around the world that have been studied less or not at all. Even for the Empire and Gribben tailings impoundments, as extensively studied as they are, it is difficult to make static and general characterizations about the tailings properties without considering spatial and temporal variations in the dynamic impoundment. Most areas in the Empire and Gribben impoundments experience one year or less without a new layer of tailings deposited on the existing surface (Vasher 1999). The ever-changing surface conditions of any active tailings impoundment make it difficult to characterize and proactively control dust susceptibility of the tailings. This is likely why most tailings impoundments utilize dust gauges dispersed around the perimeter for dust emissions monitoring and detection. When high levels of airborne particulate matter are detected in one or more gauge, tailings impoundment managers can react and apply dust suppressants in areas of concern. However, this may not be the most time efficient or cost effective way to detect and address dusting on tailings impoundments.

\subsubsection{Thermal Remote Sensing}

There are three general methods for collecting data on the natural environment (including soil properties): through laboratory testing, in-situ or field testing, and remote 
sensing. Remote sensing can be generally defined as collecting data about an object or area at some distance away from that object or area (Jensen 2009), and is more formally defined by the American Society for Photogrammetry and Remote Sensing (ASPRS) as:

...the art, science, and technology of obtaining reliable information about physical objects and the environment, through the process of recording, measuring and interpreting imagery and digital representations of energy patterns derived from non-contact sensor systems (Colwell 1997).

There are many advantages of using remote sensing to collect data, including: it does not disturb the object or area being studied (unlike in-situ testing or collecting samples for laboratory testing); it can provide greater spatial coverage rather than relying on insitu point measurements; it can remove sampling bias by collecting data systematically; and the data collected can be used for multiple purposes. However, those using remote sensing need to also understand the limitations of the data, including: ensuring that the spatial/temporal/radiometric/spectral resolution of a sensor are appropriate for the study, potentially high costs, unexpected sensor de-calibration or malfunction, and conducting appropriate data analysis (Jensen 2009).

Most remote sensing is performed to measure electromagnetic radiation (EMR). Remote sensing data can be collected by sensors on satellites, space shuttles, aircraft, unmanned aerial vehicles (UAVs), and other platforms. Remote sensing is either conducted with an active system or a passive system. Active remote sensing systems send out a pulse of EMR and have sensors to measure how much of it returns - examples of active systems include RADAR, LIDAR, and SONAR. Passive remote sensing systems, however, rely on the EMR from the sun that is emitted or reflected from a surfaceexamples of passive systems include optical cameras and thermal sensors. With the exception of ground penetrating radar, remote sensing can be used to detect surface properties only (Jensen 2009).

Thermal remote sensing relies on passively collecting thermal infrared electromagnetic energy that is emitted from objects in the 3-14 micrometer portion of the 
EMR spectrum. It should be noted, however, that due to atmospheric interference, thermal infrared EMR can only be measured in the 3-5 and 8-14 micrometer atmospheric windows. The particles of matter that make up all objects are in random motion and release kinetic energy, which can only be measured in situ. When this kinetic energy exits an object, it is converted to radiant energy, which can be measured using remote sensing. All objects with a temperature above absolute zero $(0 \mathrm{~K})$ emit electromagnetic energy in the form of thermal infrared radiant energy. The concentration of kinetic energy can be measured as kinetic temperature $\left(\mathrm{T}_{\text {kin }}\right)$, and the concentration of thermal infrared radiant energy can be measured as radiant temperature $\left(\mathrm{T}_{\mathrm{rad}}\right)$. For most objects, there is usually a high positive correlation between $\mathrm{T}_{\text {kin }}$ and $\mathrm{T}_{\mathrm{rad}}$ (always $\mathrm{T}_{\mathrm{rad}}<\mathrm{T}_{\text {kin }}$ ). For blackbodies, defined as theoretical objects that radiate energy at the maximum possible rate per unit area, $\mathrm{T}_{\mathrm{rad}}=\mathrm{T}_{\text {kin. }}$. However, for all real world materials, emissivity $(\varepsilon)$ is what describes the difference between the radiant energy of theoretical blackbodies and real-world selective radiating bodies (Jensen 2009). Emissivity is the ratio between the radiance emitted by a real-world selective radiating body and the radiance emitted by a blackbody at the same temperature and ranges from 0 to 1, with 1 being the emissivity of a blackbody (Jacob et al. 2004). Tables exist of typical emissivity values for most materials (see Jensen 2009; Lillesand et al. 2004; Sabins 2007), but emissivity can also be measured using a spectroradiometer - the spectroradiometer measures reflectance, and reflectance and emissivity add up to 1 (Vaughan et al. 2003). Utilizing the Stefan-Boltzman Law, Kirchoff's Radiation Law, and knowledge of an object's emissivity, an object's true kinetic temperature can be determined from remote sensing using the following relationship (Jensen 2009):

$$
T_{k i n}=\frac{T_{r a d}}{\varepsilon^{1 / 4}}
$$

Being able to remotely detect an object's true kinetic temperature is powerful. It enables the ability of knowing an object's temperature without having to measure it in-situ, as well as knowledge about the object's thermal properties. One such thermal property of interest is thermal inertia. 
Thermal inertia (TI) is a measure of a material or object's thermal response to temperature change. When thermal inertia is quantified for soil, and it varies with soil type and soil moisture content. It is a function of: thermal conductivity $(\lambda)$, or a measure of the rate that heat is transferred through the soil; bulk density $\left(\rho_{b}\right)$, or the mass of soil particles and water divided by the volume the particles and water occupy; and specific heat capacity (C), or a measure of the ability of the soil to absorb heat. Thermal inertia can be represented by the following relationship (Jensen 2009; Minacapilli et al. 2012):

$$
T I=\sqrt{\lambda \rho_{b} C}
$$

Thermal inertia is a physical measurement, and is not spatially or temporally constant (Jensen 2009; Minacapilli et al. 2012). For in-situ monitoring, thermal inertia requires point measurements with a thermal probe, which is not an improvement in sampling area over dust gauges currently used to evaluate the susceptibility for dusting at tailings impoundments.

An alternative method that utilizes thermal remote sensing data uses apparent thermal inertia (ATI) in lieu of thermal inertia, and Minacapilli et al. (2012) state that a direct relationship exists between TI and ATI. ATI, in its simplest form, is expressed in terms of surface albedo $(\alpha)$ and change in surface temperature $(\Delta \mathrm{T})$ from a diurnal heating cycle, which can be determined from remote sensing and are represented by:

$$
A T I=(1-\alpha) / \Delta T
$$

Change in surface temperature due to a diurnal heating cycle $(\Delta \mathrm{T})$ is straightforward and can be calculated using one daytime and one nighttime thermal image collected over the same area of interest. Using satellite thermal remote sensing requires processing relying on knowledge about the satellite and overpass time, including transforming digital numbers to brightness temperature, then to top-of-atmosphere temperature, and then to ground surface temperature if atmospheric conditions are known. Other remote sensing platforms, like UAV and airborne platforms, can also be used to collect the required thermal imagery. 
Albedo $(\alpha)$, on the other hand, is a bit more difficult to define and quantify. The simple definition of albedo is that it is the ratio of the upwelling flux to the downward flux, or the ratio between reflected radiation to incident radiation (Jensen 2009; Liang 2004). A pure white object reflects all incoming EMR over all wavelengths and has an albedo of 1, while a pure black object absorbs all incoming EMR over all wavelengths and has an albedo of 0 . Pyranometers can be used to measure flux over a range of wavelengths of EMR, which can be used to calculate albedo (Ranson et al. 1991). Spectroradiometers can be used to measure reflectance over a range of wavelengths of EMR, which can be used to calculate albedo (Feister and Grewe 1995, as can satellites with bands that measure reflectance over a range of wavelengths (Liang, 2001 \#107). Where things get complicated is trying to define the wavelengths across which reflected and incident radiation is to be measured and used to calculate albedo. For surface temperature, the appropriate wavelengths to use are clearly the thermal portion of the EMR spectrum, 3-14 micrometers. For albedo, some define the appropriate portion of the EMR spectrum to be visible and near infrared (VNIR), which is typically defined as 0.4$1.4 \mu \mathrm{m}$ (Price 1985), while others define the it to be 0.3-3.0 micrometers (Maurer 2002), 0.4-2.4 micrometers (Milton 1987), or UV/visible from 0.29-0.8 (Feister and Grewe 1995). Liang (2001); Liang (2004) mentions 10 types of albedo that can be derived from satellite data —including shortwave broadband albedo $(0.25-5.0 \mu \mathrm{m})$, visible albedo (0.4$0.7 \mu \mathrm{m})$, near-infrared albedo (0.7-5.0 $\mu \mathrm{m})$, broadband albedo (no specific definition), diffuse visible albedo (no specific definition), direct visible albedo (no specific definition), total shortwave albedo (no specific definition), direct near-infrared albedo (no specific definition), total near-infrared albedo (no specific definition), and diffuse nearinfrared albedo (no specific definition) - without providing a description of how to differentiate between some of them. It can be assumed (though it is not explicitly stated) that shortwave broadband albedo and total shortwave albedo refer to the same parameter, as do total near-infrared albedo and near infrared albedo. This brings the number of distinctly defined albedos to 8. Liang (2004) even acknowledges that the definition(s) of albedo may vary in the literature, but does not suggest a way to rectify the situation. Regardless of the definition used for albedo, using satellite remote sensing to determine 
albedo requires selecting an appropriate sensor collecting the required wavelengths and then converting spectral radiance to reflectance and then to broadband albedo. Other remote sensing platforms, like UAV and aerial platforms, could also be equipped with a suitable sensors and used to calculate albedo.

It should also be noted that ATI calculations do not take into consideration variations in atmospheric conditions between scenes. ATI is often used to detect spatial changes and distinguish boundaries between geological materials (Jensen 2009; Kahle et al. 1984), and it follows logically that it could also be used to detect temporal changes. However, Price (1985) warned that ATI is a simplified product that has limited validity but may be valuable in dry environments. Still, many studies have been conducted to find (apparent) thermal inertia for surface soil and to use (apparent) thermal inertia to determine other soil properties. Although, as pointed out by (Murray and Verhoef 2007), the relationships are often empirical in nature and/or do not account for soil thermal properties.

Multiple studies have used remote sensing to determine (apparent) thermal inertia (Murray and Verhoef 2007; Murray and Verhoef 2007; Putzig and Mellon 2007; Ramakrishnan et al. 2013; Soliman et al. 2013; Wang et al. 2010; Xue and Cracknell 1995), and Cracknell and Xue (1996) provide a thorough review of the early work performed on satellite-based mapping of thermal inertia. Many studies have also used remotely sensed (apparent) thermal inertia to indirectly determine soil moisture (Cai et al. 2005; Cai et al. 2007; Kahle et al. 1984; Liu and Zhao 2006; Minacapilli et al. 2012; Price 1980; Price 1985; Zhang et al. 2002). Details about some of the more relevant studies follow.

Xue and Cracknell (1995) developed a mathematical/physical model to derive surface soil TI/ATI from satellite data with considerations made for site-specific and environmental conditions. This TI model has been used in numerous studies, including Scheidt et al. (2010), where satellite data was used to model TI and then soil moisture content and sediment availability for arid regions, and Ramakrishnan et al. (2013) used satellite data to compare the results from Xue and Cracknell's TI model to TI determined from look up tables relating $\Delta \mathrm{T}$, albedo, and TI. Other uses of thermal inertia mapping 
using thermal remote sensing include: Soliman et al. (2013), who used airborne thermal remote sensing to determine thermal inertia based on nighttime radiation, nighttime temperature drop, duration of cooling period, and soil texture based on Murray and Verhoef (2007), was able to relate TI to soil moisture content. Others have used thermal inertia derived from thermal remote sensing collected from satellites (Verstraeten et al. 2006), airplanes (Maltese et al. 2013), and ground-based systems (Liu and Zhao 2006). While not directly applicable to this study, an exciting use of thermal inertia mapping was proposed by Putzig and Mellon (2007), who developed a methodology to map thermal inertia to predict surface heterogeneity of Mars.

No studies using thermal remote sensing to detect variations in the surface strength of soil were encountered. The closest was Scheidt et al. (2010), who used thermal inertia to predict moisture content and then wind erosion potential with sediment availability/wind threshold velocity ratio, based on the work of Fécan et al. (1998). Also, no studies used thermal remote sensing to characterize the surface of mine tailings. The closest applications of thermal remote sensing to mining encountered in this literature review was thermal remote sensing for mineral exploration (Ramakrishnan et al. 2013; Riley and Hecker 2013; Sabins 1999).

Because much of the work using thermal remote sensing to detect soil surface properties focuses on soil moisture, it is worth noting that soil moisture can also be sensed using active remote sensing, specifically RADAR (Anderson and Croft 2009; Bindlish et al. 2006; Bindlish et al. 2003; Jackson 1993; Jackson 1997). However, the data processing for RADAR remote sensing is much more intensive than that for thermal remote sensing (Jensen 2009), so this study will attempt to utilize the simpler thermal remote sensing.

Due to the high costs of airborne remote sensing missions, it is expected that this study will rely primarily on satellite-based or UAV-based thermal remote sensing paired with ground truthing. Two satellites that have demonstrated good performance for surface temperature mapping include ASTER and Landsat. ASTER, or Advanced Spaceborne Thermal Emission and Reflection Radiometer, is a collaboration between the National 
Aeronautics and Space Administration (NASA) and Japan's Ministry of Economic Trade and Industry. ASTER, launched in December of 1999, collects 14 bands of data from visible to thermal infrared, and each data scene covers $60 \mathrm{~km}$ x $60 \mathrm{~km}$. Landsat is a collaboration between NASA and USGS. Landsat has two active satellites, Landsat 7 and Landsat 8. Landsat 7, launched in April of 1999, collects 8 bands of data from visible to thermal infrared, and each data scene covers $170 \mathrm{~km}$ x $180 \mathrm{~km}$. Landsat 8, launched in February of 2013, collects 11 bands of data from visible to thermal infrared, and each data scene also covers $170 \mathrm{~km}$ x $180 \mathrm{~km}$. These satellites provide good spatial resolution - for the thermal bands, 90 meter spatial resolution for ASTER, 60 meter spatial resolution for Landsat 7, and 100 meter spatial resolution for Landsat 8. These satellites also provide good spectral resolution, with both ASTER and Landsat collecting data in more than one band that can be used for albedo and at least one band that can be used for $\Delta \mathrm{T}$. Both satellites have temporal resolutions of 16 days, but non-nadir scenes can often be obtained more frequently. Studies that demonstrated the ability to use ASTER data for surface temperature mapping include Coolbaugh et al. (2007), Katra and Lancaster (2008), Ramakrishnan et al. (2013), and Scheidt et al. (2010). Studies that demonstrated the ability to use Landsat data for surface temperature mapping include $\mathrm{Li}$ et al. (2004), Rozenstein et al. (2014), Sobrino et al. (2004), and Weng et al. (2014).

UAV technology is rapidly developing, with UAVs becoming more affordable and sensors (including thermal sensors) becoming more lightweight and UAV-friendly. The UAV industry is booming, and the application of this research demonstrates one of many ways that UAVs can be used in the future (Colomina and Molina 2014; Liu et al. 2014). No studies have been found that used UAV-based thermal remote sensing for soil surface temperature mapping, but UAV's have been used to map the surface temperature of a variety of other natural materials, like streams (Jensen et al. 2012), volcanoes (Amici et al. 2013), vegetation (Bellvert et al. 2014), and vegetation fires (Wooster et al. 2013). For the application of this specific research, a UAV that can carry the payload of a thermal sensor is all that is required to collect the thermal remote sensing data needed to determine surface temperature (a sensor to quantify albedo could also be carried, if 
desired). Benefits of UAV-based remote sensing include that imagery can be flown ondemand with any sensor that the UAV can carry, meaning that pre- and post-heating thermal imagery could be collected on the same day. Additionally, the low flying height of UAVs means that clouds do not affect data acquisition like the do for satellite data, and the spatial resolution of all imagery collected is high (often $\mathrm{cm}$ scale). However, a major drawback of UAV-based remote sensing is the intensive data processing that is required, especially if the UAV flying height varies and if the UAV imagery is collected without GPS tracking. This processing is complex because UAV-based remote sensing yields overlapping, discrete images that need to be scaled, mosaicked, and georeferenced prior to interpretation.

Satellite- or UAV-based thermal remote sensing may be a suitable alternative to monitoring dust susceptibility at tailings impoundments. Spatial and temporal variations in thermal inertia, and possibly moisture content and sediment availability, can be mapped and will provide synoptic coverage of the tailings impoundment(s).

\subsubsection{Biological Soil Crusts}

As discussed previously, wind only affects the surface of the soil, so knowledge of the shear strength and other properties of the soil surface in the upper few centimeters are most crucial when characterizing a soil's susceptibility to erosion. Additionally, surficial treatments to increase the soil's surface strength and resistance to erosion forces are required if stabilization of the sediments is to be achieved. Typical soil treatments used to increase surface strength and resist erosion rely on chemical or physical additives (Bang et al. 2009). Physical treatments involve applying a surface blanket using aggregates, prefabricated membranes or meshes, or bituminous surface treatments to protect the soil from the erosion forces. Chemical treatments can include applying magnesium chloride or calcium chloride solutions, emulsified asphalts, tree resin, or lignin derivatives to the surface of the soil to bind the soil particles together. Many of these physical and chemical treatments have environmental consequences, have high costs for application, may lose effectiveness with time due to factors like UV degradation, and/or are affected by soil moisture meaning limited applicability in arid regions (Bang et al. 2009). 
These physical and chemical treatments have varying degrees of success in helping soil to resist erosion by increasing surface strength. Especially for soils that experience cold weather dusting, chemical dust suppression has been shown to be ineffective (Vasher 1999). Rather than trying to develop new treatments, why not learn from one of nature's most successful solutions to erosion resistance, biological soil crusts?

Biological soil crusts are a specialized community of macro- and micro-biological components on or near the surface of soil (Harper and Marble 1988; West 1990). They are often referred to by many other names, including natural soil crusts, cryptogamic crusts, microbiotic crusts, cryptobiotic crusts, and microphytic crusts (Harper and Marble 1988; West 1990); biological soil crust is the most inclusive of these names and is thus the preferable term. Biological soil crusts are a distinct type of soil crust that differs from naturally-occurring inorganic physical and chemical crusts. The components of biological soil crusts can vary, but often include cyanobacteria, (green) algae, lichen, bryophytes including mosses and liverworts, microfungi, and bacteria (Belnap and Gillette 1998; West 1990). The variation in the composition of a biological soil crust can depend on many factors, including soil characteristics and the amount of disturbance (Belnap and Gillette 1998; West 1990).

Biological soil crusts can develop on the ground surface anywhere that vascular plant growth is limited or absent, but they are especially prevalent in hot or cold arid regions (Cameron and Blank 1966; Friedmann and Galun 1974; Friedmann and Ocampo-Paus 1976; Harper and Marble 1988; West 1990). They are commonly found in desert settings, but have been found located in semi-desert locations, woodlands, steppe formations, and on open ground between alpine and tundra vegetation (Belnap et al. 2001). Biological soil crusts have also been found on abandoned mine tailings, including coal tailings in New Mexico, Arizona, and North Dakota (Shubert and Starks 1980), copper tailings in China (Huang et al. 2011; Sun et al. 2004), brown coal and lignite tailings in Central Europe (Lukešová 2001), and gold tailings in South Africa (Orlekowsky et al. 2013).

Biological soil crust cover variations depend on site-specific conditions, but general trends have been documented for the effects of crust location, vascular plant cover, soil 
depth, rock cover, soil texture, soil moisture, and regional climate on crust development (Belnap et al. 2001). For example, crust cover generally decreases with increases in elevation, vascular plant cover, and soil or loose rock depth. Conversely, an increase in competent rock cover tends to lead to an increase in crust cover. Crust cover tends to increase over fine grained soils and decrease over coarse grained soils. In addition, soil moisture, soil temperature, the timing and amount of precipitation, the regional temperature range, and soil nutrients all affect the type of biological soil crust cover present.

Biological soil crusts cover otherwise bare soil in natural systems, providing an increased surface strength and resistance to wind and water erosion (Eldridge and Greene 1994). Soil featuring biological crusts that are well-developed has shown 2 to 130 times more resistance to soil erosion than bare soil or soil with crusts that are not fully developed (Belnap and Gillette 1997; Belnap et al. 2001; Leys and Eldridge 1998; McKenna Neuman et al. 1996; Williams et al. 1995). Because biological soil crusts can be composed of any combination of cyanobacteria, algae, lichen, bryophytes, microfungi, and bacteria, some crusts are stronger and more effective at resisting erosion than others. Moss crusts provide the most resistance from water and wind erosion, followed by lichen crusts, cyanobacterial/algal crusts, and bare soil (Belnap 1993; Belnap and Gardner 1993; Belnap and Gillette 1997; Belnap and Gillette 1998; Belnap et al. 2001). These differences can be attributed to the different ways in which various crust components act to resist erosion. Cyanobacteria and green algae excrete sticky polysaccharides, which binds soil particles together and creates larger soil aggregates that are more resistant to wind and water erosion. Similarly, lichen and moss rhizines bind soil particles together and form larger soil aggregates. Lichen, bryophytes, microfungi, and other crusts with higher surface profiles create a rough microtopography, which causes a still-air boundary layer to develop that protects the ground surface from wind erosion (Belnap et al. 2001).

Although cyanobacterial crusts have lower strength than many other biological soil crusts, filamentous cyanobacteria soil crusts have many other beneficial characteristics. Besides the soil particle binding that occurs from the sticky polysaccharide sheaths that 
cyanobacteria produce, cyanobacterial filaments grow between soil particles in the upper few millimeters of soil, forming a matrix that binds the soil together and holds them in place, protecting them from erosion forces (Belnap 1993; Belnap and Gardner 1993; Belnap and Gillette 1997; Cameron and Blank 1966; Friedmann and Galun 1974; Friedmann and Ocampo-Paus 1976; McKenna Neuman et al. 1996; Williams et al. 1995). Soil particles can also be attached to sites along the cyanobacteria cell wall (Eldridge and Leys 2003). Because of the soil matrix that is formed with the polysaccharide sheaths and filaments, filamentous cyanobacterial crusts can still provide significant protection from the abrasion of windborne particles (McKenna Neuman et al. 1996).

Another benefit of the establishment of biological soil crusts composed of filamentous cyanobacteria is that cyanobacteria, along with green algae, are considered to be pioneer organisms for other natural crust components (Thiet et al. 2005). Large filamentous cyanobacteria are usually the first photosynthetic species to grow on bare or disturbed soils (Ashley and Rushforth 1984; Belnap 1995; Belnap et al. 2001); this can be attributed to their ability to colonize surfaces from the air and their ability to move across soil surfaces (Schlichting 1969). The pioneer cyanobacteria form early successional crusts upon which the other biological soil crust components can grow (Thiet et al. 2005).

The reductions in soil erosion that have been documented for cyanobacterial and other biological crusts in natural systems suggest that it may be possible for similar results to be obtained from human stimulation of biological soil crusts in other locations that are susceptible to erosion. In fact, just as biological soil crusts colonize and grow on previously bare soil in natural settings unaided by human activity, the growth of biological soil crusts on previously un-crusted soil has been demonstrated successfully in the laboratory (Liu et al. 2008; McKenna Neuman and Maxwell 1999; McKenna Neuman and Maxwell 2002; McKenna Neuman et al. 2005; McKenna Neuman et al. 1996; O'Brien and McKenna Neuman 2012). Success in such applications requires a careful assessment of the soil and site conditions to select the appropriate biological soil crust component(s) to stimulate. Observing the types of crust that exist in other locations close to the site would provide a good starting point for identifying the organisms that might be 
well-suited to the soil and climate. In particular, identification of a type of filamentous cyanobacterium that can survive in the site conditions would provide a good pioneer organism to try to cultivate. The next step would be to develop cultivation techniques for large-scale application.

Although biological soil crusts are effective at reducing erosion susceptibility in-situ, and could potentially be cultivated on highly erodible soils for that purpose, they may be susceptible to disturbance, which can greatly reduce the resistance to erosion (Belnap and Gillette 1998). The degree of disturbance of biological soil crusts can range from light to heavy, with the most severe disturbance resulting in crust removal and bare soil (Belnap et al. 2001). The most common form of disturbance is in the form of compressional loading, like that from animal or pedestrian foot traffic or vehicular loading. Crusts can also be disturbed by fire or burial by sediment, which causes a loss of light and oxygen (Belnap and Eldridge 2003; Belnap et al. 2001). Whatever the form of disturbance, it can limit the biodiversity of a biological soil crust, which in turn may impact the capacity to resist erosion.

Once disturbed, biological soil crusts may require significant time spans to recover, with full recovery requiring time on the order of magnitude of years to decades to even centuries (Belnap and Eldridge 2003). Recovery time of biological soil crusts is not linear, and is therefore quite difficult to predict. Furthermore, recovery rates vary depending on environmental conditions and the degree of disturbance that the crust experienced (Belnap et al. 2001). Thus, any erosion resistance that could be gained from cultivation of biological crusts on bare or disturbed soils could only be expected to endure in locations without any disturbance.

As mentioned previously, biological soil crusts have also been found growing on abandoned mine tailings, though the literature is quite limited. Some studies have focused on the tailings from coal mining. For example, Shubert and Starks (1980) studied the relationship between coal surface mining waste and algal soil crusts on tailings in New Mexico, Arizona, and North Dakota. Lukešová (2001) studied algal and cyanobacteria crusts on brown coal and lignite tailings in Central Europe, with a focus on crust 
composition and diversity. Biological soil crusts have also been found associated with the tailings from metal ore extraction. Sun et al. (2004) studied naturally occurring biotic crusts on copper tailings in China. The crusts were found to naturally colonize the tailings between 1 and 2 years after the impoundment became inactive, and the crusts were measured to be 2-20mm thick. Huang et al. (2011) studied biological soil crusts on the same copper tailings impoundment in China that Sun et al. (2004) studied. They identified three crust types that occurred naturally and grew successfully on the tailings: algae, mixed moss-algae, and moss crusts. Finally, Orlekowsky et al. (2013) studied the cyanobacteria and algae composing biological soil crusts on both biologically rehabilitated and un-rehabilitated tailings; they found no growth on freshly deposited tailings, and much less growth on un-rehabilitated tailings as compared to tailings that were biologically rehabilitated.

All of the previous studies of biological crusts on mine tailings identified during this research were focused on ecological rehabilitation or restoration of the abandoned tailings, not on dust suppression. In addition, no studies encountered in the literature addressed biological soil crusts on active tailings impoundments, or on iron tailings in any capacity. Nevertheless, based on the available literature, it is expected that biological soil crusts might be a viable solution for the reduction of wind erosion susceptibility of iron tailings. The tailings impoundments should go undisturbed between tailings discharges if no other dust suppressants are needed, and the biological soil crust components are not expected to negatively affect tailings effluent water quality. The largest uncertainty for active tailings impoundments is if the crusts can develop sufficiently fast between tailings discharges to adequately increase the surface strength so as to reduce susceptibility to wind erosion.

\subsubsection{Surficial Strength and Erosion Susceptibility Testing}

Wind erosion mechanisms only affect the surface of the soil, so knowledge of the shear strength of the soil surface in the upper few centimeters is most crucial when characterizing a soil's susceptibility to erosion. 
There are numerous methods to determine soil strength in both laboratory and field testing. As a noted above, laboratory testing of tailings is almost always conducted on reconstituted samples, as the tailings are easily liquefiable due to their high moisture content and relatively low density and samples tend to liquefy when transported from the impoundment to the laboratory. For both the Empire and Gribben tailings, testing has been conducted in laboratory and field settings. Laboratory testing on the Empire tailings included direct shear (Arnoldt 1970; Pawloski 1979), uniaxial compression testing (Pawloski 1979; Price 1998), and triaxial testing (Arnoldt 1970; Muszynski 2000; Pawloski 1979; Wheeler 1991). Field testing on the Empire tailings included pore water pressure monitoring (King 1981), vane shear testing (Pawloski 1979), pocket penetrometer testing (Pawloski 1979), and wind tunnel testing (Vasher 1999). Laboratory testing on the Gribben tailings included uniaxial compression testing (Price 1998) and triaxial testing (Grosser 1996; Zarling 1997). No field testing was conducted on the Gribben tailings, and laboratory testing was limited, likely due to trafficability issues and the difficult nature of the tailings to handle in the laboratory (Vasher 1999).

However, these results do not necessarily represent the surface strength of the tailings and the ability to resist wind erosion. Specific to wind erosion, one of the main mechanisms to consider is saltating particle impact leading to erosion, which is not perpendicular to the soil surface (typically how soil strength testing is conducted), but is rather at a low angle to the soil surface. Methods that can be used to test the near-surface strength of tailings include penetrometer testing, vane shear testing, ball drop testing, and wind tunnel testing. None of these methods is flawless, and the details of each method follow.

Penetrometer testing involves pushing a probe into a soil sample and using it to measure the soil's resistance to penetration. Penetrometers can use probes of all shapes and sizes — needle (Humboldt 2009), flat-tip needle (Langston and McKenna Neuman 2005; McKenna Neuman and Maxwell 2002; Rice et al. 1999; Rice et al. 1997), larger diameter flat tip like pocket or Proctor penetrometer (Gerrard 1982), or cone (Gerrard 1982). Penetrometers are designed to be used at a constant rate of penetration, and can 
either measure the resistance the soil provides over a given depth of penetration (Langston and McKenna Neuman 2005; McKenna Neuman and Maxwell 2002; Rice et al. 1999; Rice et al. 1997) or measure the distance traveled over a set time of penetration (Humboldt 2009). Penetrometer testing is more accurately described as a bearing capacity test. However, because penetrometers only penetrate the surface and near-surface of the soil (depth controlled by the size of the penetrometer used), they can be used as a way to represent surface shear strength. It should be noted that, depending on the depth of penetration and the desired study depth, the inclusion of subsurface soil strength in penetrometer results may be unavoidable. Despite this limitation, there are no standard geotechnical characterization tests that physically measure the surface strength without including the influence of underlying soil, so penetrometers are the closest way to physically capture surface strength conditions. The largest benefits of penetrometer testing is that penetrometers are handheld, portable, and can be used in both laboratory and field settings. The largest issue with penetrometer testing is that penetration resistance is only a measure of relative strength, and there is not a way to use penetrometers to determine absolute strength or to even compare the results from one type of penetrometer to another (Gerrard 1982). Additionally, care should be taken when penetrometers are used to represent soil surface strength in the context of wind erosion because penetrometer testing normal to the surface is not truly representative of saltating particle impact leading to erosion, which occurs at a low angle to the soil surface; however, Langston and McKenna Neuman (2005) found that the angle of penetration did not affect the measurement of crust strength with a flat-tipped needle penetrometer.

Vane shear testing was originally designed to measure the undrained shear strength of soft clays. Vane shear testing involves a four-bladed vane being rotated at a constant rate, and the maximum applied torque/soil resistance is measured. Vane shear testing yields absolute strength rather than relative strength, though this is the absolute strength of the soil mass, not the individual soil particles. Vane shear testing leads to a high level of disturbance, as it disturbs the soil at least to the extent of the vane (a common vane diameter is $65 \mathrm{~mm}$, which is much more disturbance than handheld penetrometers 
induce) (Chandler 1988). This high level of disturbance may not be a problem for untreated tailings, but it would be a significant issue for biological soil crusts because of their sensitivity to disturbance (Belnap and Eldridge 2003; Belnap et al. 2001). While vane shear testing is traditionally a field test, there is no reason that it cannot be applied to laboratory samples as long as the samples are large enough to avoid any edge or boundary effects (Chandler 1988). No instances of studies using vane shear testing to quantify wind erosion effects for bare soil, biological soil crusts, or tailings were encountered in the literature.

To overcome the limitations of surface strength testing using penetrometers and vanes, several studies have investigated alternative ways to simply quantify surface strength. Li et al. (2010) used an air rifle to shoot a BB at a sample surface, and found a good correlation between the soil surface disturbance area due to BB impact, traditional strength measurements using a penetrometer and torvane, and threshold friction velocity (a way to quantify wind tunnel results, discussed in more detail below). Based on this work by Li et al. (2010), a novel surface strength test, called the ball drop test, was developed by Buikema et al. (accepted). In this ball drop test, a $3.4 \mathrm{~g}$ steel ball bearing with a diameter of $9.4 \mathrm{~mm}$ was dropped from a height of $1.5 \mathrm{~m}$ onto the sample surface, and the dimensions of the impact crater were measured. Buikema et al. (accepted) found this be a good way to quantify the increase in surface strength due to the presence of a calcium carbonate crust developed through MICP promoted by $S$. pasteurii. Furthermore, the ball drop results from Buikema et al. (accepted) provided more meaningful results to quantify strength increase due to the presence of a crust that those from needle penetrometer testing, because the fine-tipped needle was able to poke through the crust with limited resistance in a way that is not representative of how wind erosion occurs.

Wind tunnel testing is the most direct way to measure the effects of wind erosion on a sample. Wind tunnel testing involves placing a sample in a wind tunnel and exposing it to the direct effects of wind erosion, possibly including saltating particles, and monitoring the effects of the wind on the sample. Rather than needing to relate surface strength to dusting susceptibility, wind tunnel testing provides a direct measurement of wind erosion 
effects. With proper instrumentation, measurements that can be made with wind tunnel testing to quantify the effects of wind erosion include: sediment transport rate (Eldridge and Leys 2003); flux of sediment from the crusted surface (McKenna Neuman et al. 1996); threshold friction velocity, or the wind speed where erosion was first observed (Fécan et al. 1998; Liu et al. 2008; McKenna Neuman and Maxwell 1999); perimeter and area of crust lost (McKenna Neuman and Maxwell 1999; McKenna Neuman and Maxwell 2002; McKenna Neuman et al. 2005); and volume of crust and sediment lost (O'Brien and McKenna Neuman 2012). In addition to the air flow of the wind tunnel and the sample, a saltating particle feed can be included in wind tunnels, as most recent studies show that saltating particles are what cause soil (crust) failure and, thus, dust emissions, rather than wind alone (Langston and McKenna Neuman 2005; McKenna Neuman and Maxwell 1999; McKenna Neuman and Maxwell 2002; McKenna Neuman et al. 2005; McKenna Neuman et al. 1996; Nickling and Neuman 2009; O'Brien and McKenna Neuman 2012). Wind tunnel testing produces the most directly usable data for wind erosion studies, and wind tunnel tests can be conducted in both laboratory settings (Langston and McKenna Neuman 2005; McKenna Neuman and Maxwell 1999; McKenna Neuman and Maxwell 2002; McKenna Neuman et al. 2005; McKenna Neuman et al. 1996; O'Brien and McKenna Neuman 2012) and field settings (Price et al. 1997; Van Pelt et al. 2010; Vasher 1999). However, a different wind tunnel would be needed for laboratory and field testing. A major concern for field wind tunnel testing on a tailings impoundment would be trafficability for transporting and placing the wind tunnel, as well as spatial variability in conditions and the spatial extent of a tailings impoundment. Wind tunnels are much more costly than penetrometers or vane shear devices, so cost can be prohibitive for those without an existing wind tunnel to use. Despite the potential issues with wind tunnel availability/cost, Fécan et al. (1998) developed a theoretical relationship between threshold friction velocity and soil moisture content that was validated with laboratory data, and Scheidt et al. (2010) used this relationship to use satellite remote sensing data to predict dust susceptibility, which shows promise for relating wind tunnel data to remote sensing data. 
Once the surface strength of the untreated tailings is quantified, the strength increase due to the presence of biological soil crusts can be measured. Biological soil crusts cover otherwise bare soil, providing an increased surface strength and resistance to wind and water erosion. The fact that they can provide resistance to erosion is not contested; however, methods to quantify strength increase and measure erosion resistance increase are still being investigated and refined. There have been studies conducted in field and in the laboratory. Laboratory studies have been performed on biological soil crusts from study sites and on crusts grown from stock culture in laboratory settings. Methods to test biological soil crust strength and erosion resistance include penetrometers, which simulate the rupture of the crust under grain impact (Wang et al. 2006), and wind tunnel testing, which can provide data on soil loss and crust disturbance due to wind erosion (Langston and McKenna Neuman 2005). In addition, because biological soil crusts can carry tensile loads, unlike bare soil or tailings, they can also be tested using bending and tensile tests. Bending tests measure the stress-strain behavior and stiffness of crusts and represent the fiber stress and the mechanism of crust flaking/fatigue (McKenna Neuman et al. 1996). Tensile tests also measure the stress-strain behavior of crusts (Wang et al. 2006). However, while bending and tensile tests can be used to compare the stress-strain behavior of different biological soil crusts, they cannot be used to directly compare to untreated soil or tailings. Finally, it may also be possible to model the erosion resistance of biological soil crusts with membrane theory (Otto and Trostel 1962), because some crusts serve as a membrane to resist grain impact.

Field testing to quantify the strength and increase erosion resistance of in-situ biological soil crusts has been conducted at sites such as the cyanobacterial soil crusts in the semi-arid Kalahari rangeland of Africa (Thomas and Dougill 2007), the microbiotic crusts in the high equatorial Andes of Venezuela (Pérez 1997), and the biological soil crusts in the northwestern Negev dune field along the Israel-Egypt border (Zaady et al. 2010). These field testing campaigns included penetrometer testing (Pérez 1997; Thomas and Dougill 2007; Zaady et al. 2010) and indicated that the biological soil crusts tested had higher resistance to wind erosion (penetrometer) than soil lacking a crust. 
Laboratory testing to quantify the strength increase and erosion resistance of biological soil crusts has been conducted on both biological soil crusts from study sites and crusts grown from stock culture in laboratory settings. Crusts collected for laboratory testing included: a microbiotic crust from Shapotou, China (Wang et al. 2006); a biological soil crust dominated by lichen and moss from the Mallee Region in southeast Australia (Eldridge and Leys 2003); a biological soil crust containing cyanobacteria and some lichen and/or moss from the Chihuahan Desert in south-central New Mexico (Belnap and Gillette 1998); and an algal crust from the southease region of the Tengger Desert in China (Liu et al. 2008). The strength characterization testing included the tensile test (Wang et al. 2006) and wind tunnel testing (Belnap and Gillette 1998; Eldridge and Leys 2003). Wind tunnel testing of the sampled crusts gave information of the sediment transport rate of crusted and un-crusted soils (Eldridge and Leys 2003), the threshold friction velocity (wind speed where erosion was first noted) of the crusts (Liu et al. 2008), and indicated that soils with well-developed soil crusts are able to resist wind erosion better than un-crusted soils (Belnap and Gillette 1998). Biological soil crusts grown from stock cultures in the laboratory included algal crusts (McKenna Neuman et al. 1996), fungal crusts (McKenna Neuman and Maxwell 1999; McKenna Neuman et al. 2005), moss crusts (McKenna Neuman and Maxwell 2002; McKenna Neuman et al. 2005), and cyanobacteria crusts (Liu et al. 2008; O'Brien and McKenna Neuman 2012). All crusts were tested using a wind tunnel featuring saltating particles, which represents the primary eroding mechanism of wind (Liu et al. 2008; McKenna Neuman and Maxwell 1999; McKenna Neuman and Maxwell 2002; McKenna Neuman et al. 2005; McKenna Neuman et al. 1996; O'Brien and McKenna Neuman 2012). The measurements made during the wind tunnel testing of these biological soil crusts varied, and the results were reported in various ways, including: flux of sediment from the crusted surface (McKenna Neuman et al. 1996); threshold friction velocity, or the wind speed where erosion was first observed (Liu et al. 2008; McKenna Neuman and Maxwell 1999); perimeter and area of crust lost (McKenna Neuman and Maxwell 1999; McKenna Neuman and Maxwell 2002; McKenna Neuman et al. 2005); and volume of crust and sediment lost (O'Brien and McKenna Neuman 2012). The strength of several crusts was 
also quantified using the bending test (Liu et al. 2008; McKenna Neuman et al. 1996) or a penetrometer (McKenna Neuman and Maxwell 2002). In general, these studies all indicated that the presence of biological soil crusts provides an increase in strength and a decrease in wind erosion, when compared to un-crusted soil.

There are certainly benefits of field testing to determine the erosion resistance of biological crusts. For example, testing crusts in-situ means that there is no sample disturbance. However, penetrometers only measure relative strength (Gerrard 1982). Penetration resistance must be correlated with wind tunnel data to determine wind erosion resistance because the flexibility of biological soil crusts helps them resist saltating particles, meaning that penetration resistance might not fully represent the crust's ability to resist wind erosion (O'Brien and McKenna Neuman 2012). For wind erosion susceptibility, the correlation of field strength data (penetrometer) and laboratory erosion resistance (wind tunnel) would yield the best site-specific characterization; if sitespecifics are not a concern, samples grown from stock cultures and only tested in laboratory settings should yield sufficient results.

Based on the existing literature, for this study, the strength of untreated tailings was characterized with penetrometer, vane shear, and ball drop testing in the laboratory and field paired with laboratory wind tunnel testing to quantify (relative) surface strength and erosion susceptibility. Then, laboratory samples of tailings covered with biological soil crusts were also subjected to ball drop and wind tunnel testing to quantify (relative) surface strength increase and erosion susceptibility decrease.

\subsubsection{Literature Synthesis}

Soil erosion is a concern to both environmental and human health. The issue of wind erosion and dust susceptibility has been studied extensively for agricultural and desert soils, though much more emphasis has been placed on warm weather dusting rather than cold weather dusting. These agricultural and desert soils differ from mine tailings because of their organic and clay mineral content. Mine tailings are a relatively homogeneous material with similar mineralogy, yet with moisture, density, and grain size varying spatially and temporally across an impoundment. Only one research group has 
investigated the issue of wind erosion specifically on tailings impoundments (Price et al. 1997; Vasher 1999). These studies on tailings impoundments did not investigate methods of proactively detecting and controlling dust emissions as an alternative to the reactive method of using dust gauges to detect airborne particulate matter. Also, these studies did not investigate the effectiveness of alternative dust suppressants that may be longer lasting and less detrimental to the environment.

The application of using thermal remote sensing to detect spatial and temporal changes in soil surface properties, like (apparent) thermal inertia, moisture content, and strength, has also been studied extensively. However, no studies were encountered that used thermal remote sensing to detect variations in surface conditions for tailings impoundments. The fact that remote sensing can only yield surface measurements is acceptable, because wind erosion is a surficial process. In fact, remote sensing techniques are able to capture surface properties in a way that traditional, physical measurements of moisture content and strength are not, and capturing surface conditions is desirable for studying wind erosion. Thermal remote sensing should enable detection of spatial and temporal changes in (apparent) thermal inertia, strength, moisture content, or dust susceptibility for mine tailings, because it has been shown to do the same for other soils.

Biological soil crusts have been shown to increase the surface strength and decrease the erosion susceptibility of soil in both field and laboratory testing. A limited number of studies have also identified biological soil crust growth on inactive tailings impoundments, but the focus of these studies was on ecological rehabilitation of the tailings rather than on dust suppression. No studies were encountered that addressed a strength increase and dust susceptibility decrease for tailings covered with a biological soil crust, nor were there any studies that addressed growth of biological soil crusts on active impoundments. A study of the effects of biological soil crust growth on tailings surface strength and dust susceptibility could utilize a combination of field strength testing and laboratory wind tunnel testing. 


\subsection{References}

Amici, S., Turci, M., Giammanco, S., Spampinato, L., and Giulietti, F. (2013). "UAV thermal infrared remote sensing of an Italian mud volcano." Advances in Remote Sensing, 2013.

Anderson, K., and Croft, H. (2009). "Remote sensing of soil surface properties." Progress in Physical Geography, 33(4), 457-473.

Arnoldt, R. O. (1970). "Development of pore water pressure in a fine grained iron ore tailings sample." MS, Michigan Technological University.

Ashley, J., and Rushforth, S. (1984). "Growth of soil algae on top soil and processed oil shale from the Uintah Basin, Utah, USA." Reclamation \& revegetation research, 3(1), 49-63.

Bang, S. C., and Bang, S. S. (2011). "KGS Awards Lectures: Application of Microbiologically Induced Soil Stabilization Technique for Dust Suppression." International Journal of Geo-Engineering, 3(2), 27-37.

Bang, S. S., Bang, S., Frutiger, S., Nehl, L. M., and Comes, B. L. "Application of novel biological technique in dust suppression." Proc., Transportation Research Board 88th Annual Meeting.

Barnes, D., and Connor, B. (2014). "Managing Dust on Unpaved Roads and Airports." Alaska University Transportation Center and Alaska Department of Transportation, Fairbanks, AK.

Bellvert, J., Zarco-Tejada, P. J., Girona, J., and Fereres, E. (2014). "Mapping crop water stress index in a 'Pinot-noir'vineyard: comparing ground measurements with thermal remote sensing imagery from an unmanned aerial vehicle." Precision agriculture, 15(4), 361-376.

Belnap, J. (1993). "Recovery rates of cryptobiotic crusts: inoculant use and assessment methods." Western North American Naturalist, 53(1), 89-95.

Belnap, J. (1995). "Surface disturbances: Their role in accelerating desertification." Environmental monitoring and assessment, 37(1-3), 39-57.

Belnap, J., and Eldridge, D. (2003). "Disturbance and recovery of biological soil crusts." Biological soil crusts: structure, function, and management, Springer, 363-383.

Belnap, J., and Gardner, J. S. (1993). "Soil microstructure in soils of the Colorado Plateau: the role of the cyanobacterium Microcoleus vaginatus." Western North American Naturalist, 53(1), 40-47.

Belnap, J., and Gillette, D. (1997). "Disturbance of biological soil crusts: impacts on potential wind erodibility of sandy desert soils in southeastern Utah." Land Degradation \& Development, 8(4), 355-362.

Belnap, J., and Gillette, D. A. (1998). "Vulnerability of desert biological soil crusts to wind erosion: the influences of crust development, soil texture, and disturbance." Journal of Arid Environments, 39(2), 133-142.

Belnap, J., Laxalt, M., and Peterson, P. (2001). Biological soil crusts: ecology and management, US Department of the Interior, Bureau of Land Management, National Science and Technology Center, Information and Communications Group. 
Bindlish, R., Jackson, T. J., Gasiewski, A. J., Klein, M., and Njoku, E. G. (2006). "Soil moisture mapping and AMSR-E validation using the PSR in SMEX02." Remote Sensing of Environment, 103(2), 127-139.

Bindlish, R., Jackson, T. J., Wood, E., Gao, H., Starks, P., Bosch, D., and Lakshmi, V. (2003). "Soil moisture estimates from TRMM Microwave Imager observations over the Southern United States." Remote Sensing of Environment, 85(4), 507515.

Buck, S., and Gerard, D. (2001). "Cleaning Up Mining Waste." Political Economy Research Center Research Study.

Buikema, N. D., Zwissler, B. E., Seagren, E. A., Oommen, T., and Vitton, S. J. (accepted). "Stabilization of Iron Mine Tailings through Biocalcification." Environmental Geotechnics.

Cai, G., Wu, J., Xue, Y., Hu, Y., Guo, J., and Tang, J. "Soil moisture retrieval from MODIS data in northern china plain using thermal inertia model (SoA-TI)." Proc., Geoscience and Remote Sensing Symposium, 2005. IGARSS'05. Proceedings. 2005 IEEE International, IEEE, 4501-4504.

Cai, G., Xue, Y., Hu, Y., Wang, Y., Guo, J., Luo, Y., Wu, C., Zhong, S., and Qi, S. (2007). "Soil moisture retrieval from MODIS data in Northern China Plain using thermal inertia model." International journal of remote sensing, 28(16), 35673581.

Cameron, R. E., and Blank, G. B. (1966). "Desert algae: soil crusts and diaphanous substrata as algal habitats."

Chandler, R. J. (1988). "The in-situ measurement of the undrained shear strength of clays using the field vane." Vane shear strength testing in soils: field and laboratory studies, 13-44.

Chu, J., Stabnikov, V., and Ivanov, V. (2012). "Microbially induced calcium carbonate precipitation on surface or in the bulk of soil." Geomicrobiology Journal, 29(6), 544-549.

Colomina, I., and Molina, P. (2014). "Unmanned aerial systems for photogrammetry and remote sensing: A review." ISPRS Journal of Photogrammetry and Remote Sensing, 92, 79-97.

Colwell, R. N. (1997). "History and place of photographic interpretation." Manual of photographic interpretation, 2, 33-48.

Coolbaugh, M., Kratt, C., Fallacaro, A., Calvin, W., and Taranik, J. (2007). "Detection of geothermal anomalies using advanced spaceborne thermal emission and reflection radiometer (ASTER) thermal infrared images at Bradys Hot Springs, Nevada, USA." Remote Sensing of Environment, 106(3), 350-359.

Cracknell, A., and Xue, Y. (1996). "Thermal inertia determination from space—a tutorial review." International Journal of Remote Sensing, 17(3), 431-461.

Davies, M., Martin, T., and Lighthall, P. (2000). "Tailings dam stability: essential ingredients for success." Slope Stability in Surface Mining, 365-377.

Davies, M. P., and Rice, S. (2001). "An alternative to conventional tailings management"dry stack" filtered tailings." Proceeding of Tailings and Mine Waste'01, 411420 . 
DeJong, J., Soga, K., Kavazanjian, E., Burns, S., AL QABANY, A., AYDILEK, A., BANG, S., BURBANK, M., CASLAKE, L., and CHEN, C. (2013).

"Biogeochemical processes and geotechnical applications: progress, opportunities and challenges." Geotechnique, 63(4), 287-301.

DeJong, J. T., Fritzges, M. B., and Nüsslein, K. (2006). "Microbially induced cementation to control sand response to undrained shear." Journal of Geotechnical and Geoenvironmental Engineering, 132(11), 1381-1392.

Duncan, J. M., and Wright, S. G. (2005). Soil strength and slope stability.

Eldridge, D., and Greene, R. (1994). "Microbiotic soil crusts-a review of their roles in soil and ecological processes in the rangelands of Australia." Soil Research, 32(3), 389-415.

Fécan, F., Marticorena, B., and Bergametti, G. "Parametrization of the increase of the aeolian erosion threshold wind friction velocity due to soil moisture for arid and semi-arid areas." Proc., Annales Geophysicae, Springer, 149-157.

Feister, U., and Grewe, R. (1995). "Spectral albedo measurements in the UV and visible region over different types of surfaces." Photochemistry and Photobiology, 62(4), 736-744.

Friedmann, E. I., and Galun, M. (1974). "Desert algae, lichens and fungi." Desert biology, 2, 165-212.

Friedmann, E. I., and Ocampo-Paus, R. (1976). "Endolithic blue-green algae in the Dry Valley: primary producers in the Antarctic Desert ecosystem." Science, 193, 1247-1249.

Gatto, L. W. (1995). "Soil Freeze-Thaw Effects on Bank Erodibility and Stability."US Army Corps of Engineers Cold Regions Research \& Engineering Laboratory, Hanover, NH.

Gerrard, J. (1982). "The use of hand-operated soil penetrometers." Area, 227-234.

Grannemann, N. (1984). "Hydrogeology and effects of tailings basins on the hydrology of Sands Plain, Marquette County, Michigan." United States Geological Survey.

Greeley, R., and Iversen, J. D. (1987). Wind as a geological process: on Earth, Mars, Venus and Titan, CUP Archive.

Grosser, A. T. (1996). "Normally consolidated sample preparation of hematite mine tailings for triaxial testing." MS, Michigan Technological University.

Harper, K. T., and Marble, J. R. (1988). "A role for nonvascular plants in management of arid and semiarid rangelands." Vegetation science applications for rangeland analysis and management, Springer, 135-169.

Hoffert, J. R. (1947). "Acid mine drainage." Industrial \& Engineering Chemistry, 39(5), 642-646.

Huang, L. N., Tang, F. Z., Song, Y. S., Wan, C. Y., Wang, S. L., Liu, W. Q., and Shu, W. S. (2011). "Biodiversity, abundance, and activity of nitrogen - fixing bacteria during primary succession on a copper mine tailings." FEMS microbiology ecology, 78(3), 439-450.

Huang, S. L., and Aughenbaugh, N. B. (1987). "Sublimation of pore ice in frozen silt." Journal of Cold Regions Engineering, 1(4), 171-181. 
Humboldt (2009). "Universal and Portable Penetrometers."

$<$ http://www.humboldtmfg.com/manuals/H-1200 H-1250 H-

1252 man 0709.pdf>.

Huszar, P. C., and Piper, S. L. (1986). "Estimating the off-site costs of wind erosion in New Mexico." Journal of soil and water conservation, 41(6), 414-416.

Jackson, T. J. (1993). "Measuring surface soil moisture using passive microwave remote sensing." Hydrological processes, 7(2), 139-152.

Jackson, T. J. (1997). "Soil moisture estimation using special satellite microwave/imager satellite data over a grassland region." Water Resources Research, 33(6), 14751484.

Jacob, F., Petitcolin, F. o., Schmugge, T., Vermote, E., French, A., and Ogawa, K. (2004). "Comparison of land surface emissivity and radiometric temperature derived from MODIS and ASTER sensors." Remote Sensing of Environment, 90(2), 137-152.

Jensen, A. M., Neilson, B. T., McKee, M., and Chen, Y. "Thermal remote sensing with an autonomous unmanned aerial remote sensing platform for surface stream temperatures." Proc., 2012 IEEE International Geoscience and Remote Sensing Symposium, IEEE, 5049-5052.

Jensen, J. R. (2009). Remote Sensing of the Environment: An Earth Resource Perspective Pearson Education India.

Kahle, A. B., Schieldge, J. P., and Alley, R. E. (1984). "Sensitivity of thermal inertia calculations to variations in environmental factors." Remote Sensing of Environment, 16(3), 211-232.

Katra, I., and Lancaster, N. (2008). "Surface-sediment dynamics in a dust source from spaceborne multispectral thermal infrared data." Remote sensing of Environment, 112(7), 3212-3221.

King, W. B. (1981). "Observations and analysis of the 1979 experimental tailings dam at Empire Tailings Basin One." MS, Michigan Technological University.

Lal, R. (1994). "Soil erosion by wind and water: problems and prospects." Soil erosion research methods, 2, 1-9.

Langston, G., and McKenna Neuman, C. (2005). "An experimental study on the susceptibility of crusted surfaces to wind erosion: a comparison of the strength properties of biotic and salt crusts." Geomorphology, 72(1), 40-53.

Leys, J. F., and Eldridge, D. J. (1998). "Influence of cryptogamic crust disturbance to wind erosion on sand and loam rangeland soils." Earth Surf. Process. Landf., 23(11), 963-974.

Li, F., Jackson, T. J., Kustas, W. P., Schmugge, T. J., French, A. N., Cosh, M. H., and Bindlish, R. (2004). "Deriving land surface temperature from Landsat 5 and 7 during SMEX02/SMACEX." Remote sensing of environment, 92(4), 521-534.

Li, J., Okin, G. S., Herrick, J. E., Belnap, J., Munson, S. M., and Miller, M. E. (2010). "A simple method to estimate threshold friction velocity of wind erosion in the field." Geophysical Research Letters, 37(10).

Liang, S. (2001). "Narrowband to broadband conversions of land surface albedo I: Algorithms." Remote Sensing of Environment, 76(2), 213-238. 
Liang, S. (2004). Quantitative remote sensing of land surfaces, John Wiley \& Sons, Hoboken, NJ.

Lillesand, T. M., Kiefer, R. W., and Chipman, J. W. (2004). Remote sensing and image interpretation, John Wiley \& Sons Ltd.

Liu, P., Chen, A. Y., Huang, Y.-N., Han, J.-Y., Lai, J.-S., Kang, S.-C., Wu, T.-H., Wen, M.-C., and Tsai, M.-H. (2014). "A review of rotorcraft Unmanned Aerial Vehicle (UAV) developments and applications in civil engineering." SMART STRUCTURES AND SYSTEMS, 13(6), 1065-1094.

Liu, Y., Cockell, C. S., Wang, G., Hu, C., Chen, L., and De Philippis, R. (2008). "Control of Lunar and Martian dust-experimental insights from artificial and natural cyanobacterial and algal crusts in the desert of Inner Mongolia, China." Astrobiology, 8(1), 75-86.

Liu, Z., and Zhao, Y. (2006). "Research on the method for retrieving soil moisture using thermal inertia model." Science in China Series D, 49(5), 539-545.

Lukešová, A. (2001). "Soil Algae in Brown Coal and Lignite Post - Mining Areas in Central Europe (Czech Republic and Germany)." Restoration Ecology, 9(4), 341350.

Maltese, A., Capodici, F., Ciraolo, G., and La Loggia, G. (2013). "Mapping soil water content under sparse vegetation and changeable sky conditions: comparison of two thermal inertia approaches." Journal of Applied Remote Sensing, 7(1), 073548-073548.

Marticorena, B., and Bergametti, G. (1995). "Modeling the atmospheric dust cycle: 1. Design of a soil-derived dust emission scheme." Journal of Geophysical Research, 100(D8), 16415-16416,16430.

Maurer, J. (2002). "Retrieval of surface albedo from space."

McKenna Neuman, C., and Maxwell, C. (1999). "A wind tunnel study of the resilience of three fungal crusts to particle abrasion during aeolian sediment transport." Catena, 38(2), 151-173.

McKenna Neuman, C., and Maxwell, C. (2002). "Temporal aspects of the abrasion of microphytic crusts under grain impact." Earth Surf. Process. Landf., 27(8), 891908.

McKenna Neuman, C., Maxwell, C., and Rutledge, C. (2005). "Spatial and temporal analysis of crust deterioration under particle impact." Journal of Arid Environments, 60(2), 321-342.

McKenna Neuman, C., Maxwell, C. D., and Boulton, J. W. (1996). "Wind transport of sand surfaces crusted with photoautotrophic microorganisms." Catena, 27(3), 229-247.

Michalowski, R. L., and Zhu, M. (2006). "Frost heave modelling using porosity rate function." International journal for numerical and analytical methods in geomechanics, 30(8), 703-722.

Milton, E. (1987). "Principles of field spectroscopy." Remote Sensing, 8(12), 1807-1827. Minacapilli, M., Cammalleri, C., Ciraolo, G., D'Asaro, F., Iovino, M., and Maltese, A. (2012). "Thermal inertia modeling for soil surface water content estimation: A laboratory experiment." Soil Science Society of America Journal, 76(1), 92-100. 
Morgan, R. P. C. (2009). Soil erosion and conservation, John Wiley \& Sons.

Murray, T., and Verhoef, A. (2007). "Moving towards a more mechanistic approach in the determination of soil heat flux from remote measurements: I. A universal approach to calculate thermal inertia." Agricultural and Forest Meteorology, $147(1), 80-87$.

Murray, T., and Verhoef, A. (2007). "Moving towards a more mechanistic approach in the determination of soil heat flux from remote measurements: II. Diurnal shape of soil heat flux." Agricultural and forest meteorology, 147(1), 88-97.

Muszynski, M. R. (2000). "Void ratio distribution of normally consolidated coarsegrained magnetite tailings as a funcion of aging time." MS, Michigan Technological University.

Newton, S. M. (1981). "One-dimensional compression of non plastic silt size mine tailings." MS, Michigan Technological University.

Nickling, W. G., and McKenna Neuman, C. (2009). "Aeolian sediment transport." Geomorphology of Desert Environments, Springer, 517-555.

O'Brien, P., and McKenna Neuman, C. (2012). "A wind tunnel study of particle kinematics during crust rupture and erosion." Geomorphology, 173, 149-160.

Orlekowsky, T., Venter, A., van Wyk, F., and Levanets, A. (2013). "Cyanobacteria and algae of gold mine tailings in the Northwest Province of South Africa." Nova Hedwigia, 97(3-4), 281-294.

Otto, F., and Trostel, R. (1962). "Tensile Structures, Vol. I." Massachusetts institute of Technology, Cambridge.

Pawloski, A. R. (1979). "An investigation of the stability of a mine tailings embankment." MS, Michigan Technological University.

Pérez, F. L. (1997). "Microbiotic crusts in the high equatorial Andes, and their influence on paramo soils." Catena, 31(3), 173-198.

Price, J. C. (1980). "The potential of remotely sensed thermal infrared data to infer surface soil moisture and evaporation." Water Resources Research, 16(4), 787795.

Price, J. C. (1985). "On the analysis of thermal infrared imagery: the limited utility of apparent thermal inertia." Remote sensing of Environment, 18(1), 59-73.

Price, J. C. (1998). "Evaluation and characterization of the effectiveness of dust suppressants on iron ore mine tailings." Michigan Technological University.

Price, J. C., Vasher, D. A., Vitton, S. J., and Paterson, K. (1997). "Characterization of fugitive dust emissions from a northern Michigan iron mine." Tailings and Mine Waste '98, Balkema Publishers, Rotterdam, Netherlands, Fort Collins, CO, 743751.

Priest, J. L. (1997). "Determining the compressibility characteristics of a silt from the Tilden Mine." MS, Michigan Technological University.

Putzig, N. E., and Mellon, M. T. (2007). "Apparent thermal inertia and the surface heterogeneity of Mars." Icarus, 191(1), 68-94.

Ramakrishnan, D., Bharti, R., Singh, K., and Nithya, M. (2013). "Thermal inertia mapping and its application in mineral exploration: results from Mamandur polymetal prospect, India." Geophysical Journal International, 195(1), 357-368. 
Ranson, K., Irons, J., and Daughtry, C. (1991). "Surface albedo from bidirectional reflectance." Remote sensing of Environment, 35(2), 201-211.

Rice, M., McEwan, I., and Mullins, C. (1999). "A conceptual model of wind erosion of soil surfaces by saltating particles." Earth Surf. Process. Landf., 24(5), 383-392.

Rice, M., Mullins, C., and McEwan, I. (1997). "An analysis of soil crust strength in relation to potential abrasion by saltating particles." Earth Surf. Process. Landf., 22(9), 869-883.

Riley, D. N., and Hecker, C. A. (2013). "Mineral Mapping with Airborne Hyperspectral Thermal Infrared Remote Sensing at Cuprite, Nevada, USA." Thermal Infrared Remote Sensing, Springer, 495-514.

Rozenstein, O., Qin, Z., Derimian, Y., and Karnieli, A. (2014). "Derivation of Land Surface Temperature for Landsat-8 TIRS Using a Split Window Algorithm." Sensors, 14(4), 5768-5780.

Sabins, F. F. (1999). "Remote sensing for mineral exploration." Ore Geology Reviews, 14(3), 157-183.

Sabins, F. F. (2007). Remote sensing: principles and applications, Waveland Press.

Scheidt, S., Ramsey, M., and Lancaster, N. (2010). "Determining soil moisture and sediment availability at White Sands Dune Field, New Mexico, from apparent thermal inertia data." Journal of Geophysical Research: Earth Surface (20032012), 115.

Scherer, K., and Ketzenberger, B. (2014). "Fugitive Dust Management at Cliff's Michigan Operations." B. Zwissler, ed.

Schlichting, H. E., Jr. (1969). "The importance of airborne algae and protozoa." Journal of the Air Pollution Control Association, 19(12), 946-951.

Shubert, L. E., and Starks, T. L. (1980). "Soil-algal relationships from surface mined soils." British Phycological Journal, 15(4), 417-428.

Sobrino, J. A., Jiménez-Muñoz, J. C., and Paolini, L. (2004). "Land surface temperature retrieval from LANDSAT TM 5." Remote Sensing of environment, 90(4), 434440.

Soliman, A., Heck, R. J., Brenning, A., Brown, R., and Miller, S. (2013). "Remote sensing of soil moisture in vineyards using airborne and ground-based thermal inertia Data." Remote Sensing, 5(8), 3729-3748.

Stocks-Fischer, S., Galinat, J. K., and Bang, S. S. (1999). "Microbiological precipitation of $\mathrm{CaCO}<\mathrm{sub}>3</$ sub $>$." Soil Biology and Biochemistry, 31(11), 1563-1571.

Sun, Q., An, S., Yang, L., and Wang, Z. (2004). "Chemical properties of the upper tailings beneath biotic crusts." Ecological Engineering, 23(1), 47-53.

Theriault, J., Frostiak, J., and Welch, D. "Surface disposal of paste tailings at the Bulyanhulu gold mine, Tanzania." Proc., Proceedings of Sudbury, 265-269.

Thiet, R. K., Boerner, R., Nagy, M., and Jardine, R. (2005). "The effect of biological soil crusts on throughput of rainwater and N into Lake Michigan sand dune soils." Plant and soil, 278(1-2), 235-251.

Thomas, A. D., and Dougill, A. J. (2007). "Spatial and temporal distribution of cyanobacterial soil crusts in the Kalahari: implications for soil surface properties." Geomorphology, 85(1), 17-29. 
USEPA (1994). "Technical Report: Design and Evaluation of Tailings Dams."Washington, DC.

USEPA (2012). "Particulate Matter (PM-10)." $<$ http://www.epa.gov/airtrends/aqtrnd95/pm10.html $>$.

USEPA (2014). "Fine Particule Matter (PM2.5) Designations." $<$ http://www.epa.gov/pmdesignations/ $>$.

USEPA (2014). "Mine Waste Technology." < http://www.epa.gov/nrmrl/std/mwt/>.

Van Pelt, R., Zobeck, T., Baddock, M., and Cox, J. (2010). "Design, construction, and calibration of a portable boundary layer wind tunnel for field use." Transactions of the ASABE, 53(5), 1413-1422.

Vasher, D. R. (1999). "A technique for assessing fugitive particulate emission potential from an active mine tailings basin." Michigan Technological University.

Vaughan, R. G., Calvin, W. M., and Taranik, J. V. (2003). "SEBASS hyperspectral thermal infrared data: surface emissivity measurement and mineral mapping." Remote Sensing of Environment, 85(1), 48-63.

Verstraeten, W. W., Veroustraete, F., van der Sande, C. J., Grootaers, I., and Feyen, J. (2006). "Soil moisture retrieval using thermal inertia, determined with visible and thermal spaceborne data, validated for European forests." Remote Sensing of Environment, 101(3), 299-314.

Vick, S. G. (1983). Planning, Design, and Analysis of Tailings Dams, John Wiley \& Sons, Inc., New York, NY.

Wang, J., Bras, R., Sivandran, G., and Knox, R. (2010). "A simple method for the estimation of thermal inertia." Geophysical Research Letters, 37(5).

Wang, Z.-T., Zhou, Y.-H., and Zheng, X.-J. (2006). "Tensile test of natural microbiotic crust." Catena, 67(2), 139-143.

Weng, Q., Fu, P., and Gao, F. (2014). "Generating daily land surface temperature at Landsat resolution by fusing Landsat and MODIS data." Remote Sensing of Environment, 145, 55-67.

West, N. E. (1990). "Structure and functions of microphytic soil crusts in wildland ecosystems of arid to semi-arid regions." Advances in ecological Research, 20, $179-223$.

Wheeler, M. J. (1991). "Stress-strain characteristics of normally consolidated iron ore mine tailings during undrained shear." MS, Michigan Technological University.

Whiffin, V. S., van Paassen, L. A., and Harkes, M. P. (2007). "Microbial carbonate precipitation as a soil improvement technique." Geomicrobiology Journal, 24(5), 417-423.

Williams, J., Dobrowolski, J., West, N., and Gillette, D. (1995). "Microphytic crust influence on wind erosion." Transactions of the ASAE, 38(1), 131-137.

Wooster, M. J., Roberts, G., Smith, A. M., Johnston, J., Freeborn, P., Amici, S., and Hudak, A. T. (2013). "Thermal remote sensing of active vegetation fires and biomass burning events." Thermal Infrared Remote Sensing, Springer, 347-390.

Xue, Y., and Cracknell, A. (1995). "Advanced thermal inertia modelling." Remote Sensing, 16(3), 431-446. 
Zaady, E., Ben-David, E. A., Sher, Y., Tzirkin, R., and Nejidat, A. (2010). "Inferring biological soil crust successional stage using combined PLFA, DGGE, physical and biophysiological analyses." Soil Biology and Biochemistry, 42(5), 842-849.

Zarling, J. C. (1997). "The characteristics of normally consolidated hematite mine tailings during drained and undrained shear." MS, Michigan Technological University.

Zhang, R., Sun, X., and Zhu, Z. (2002). "Remote sensing information model in surface evaporation from differential thermal inertia and it's validation in Gansu Province." Science in China (Series D), 32, 1041-1050. 



\section{Chapter 2 Detailed Laboratory Methods}

This chapter outlines the methods used to accomplish the four project objectives. This chapter begins with a description of the general experimental materials and methods to be used for achieving these four objectives, followed by the specific methods used for each objective.

\subsection{General Materials and Methods}

\subsubsection{Tailings Samples}

All laboratory testing was conducted with samples of tailings obtained from iron mine tailings impoundments in the region. Specifically, iron tailings samples were used from the Empire Mine (located in Palmer, MI as shown in Figure 2-1), the Tilden Mine/Gribben Impoundment (located in Palmer, MI, also shown in Figure 2-1), and/or the United Taconite Mine (located in Forbes, MN, see Figure 2-2). The magnetite tailings from the Empire mine are referred to as MI-magnetite tailings in this dissertation, or $M I$ mag for short, the magnetite tailings from the United Taconite mine are referred to as $M N$-magnetite tailings, or $M N$-mag for short, and the hematite tailings from the Tilden mine will be referred to as MI-hematite tailings, or MI-hem for short.

Samples of tailings from all listed mines were obtained and characterized for general geotechnical properties, including specific gravity and grain size distribution, as summarized in Table 2-1. The samples summarized in Table 2-1 were collected using a shovel and bucket from various locations around each impoundment at various distances from discharge points, and were selected to capture a wide variety of grain sizes, which explains why the standard deviations for grain size fractions are so high.

The samples selected for use in laboratory testing to satisfy Objectives 1, 3, and 4 were primarily silt-sized, because this is the particle size of concern with regards to dust emissions and sublimation. Due to resource limitations, the same sample could not be used for all lab analyses to satisfy these objectives, but the samples that were used were 
chosen because of their similar grain size distributions. The specific geotechnical properties for samples used in laboratory testing are summarized in Figure 2-3.

Because of the aforementioned limited supply of tailings, and because of the desire to use samples with a similar grain size distribution, resource management of the tailings was important. Tailings used to satisfy Objectives 1 and 3 could be reused because no treatments were applied to the tailings during laboratory testing. However, tailings used to satisfy Objective 4 could not be reused due to the biological treatments being applied. Therefore, there was a concern about having sufficient tailings from the same sample for all biological treatments, which was deemed necessary to have comparable results between treatment types. To overcome this, several MI-mag and MN-mag samples were blended to ensure enough material was available to prepare consistent samples for biological treatment. This blending procedure is described below.

Four individual tailings samples, two from MI-mag and two from MN-mag, were air dried for at least 2-3 weeks, and then large soil aggregates that formed in the drying process were broken down or "crushed" into individual tailings particles with a MicroDeval apparatus (ASTM D7428-15). The Micro-Deval cylinder was filled approximately halfway with air-dried tailings, 10 steel ball bearings were added, and the device was run for 10 minutes. After crushing, the tailings were emptied, and this was repeated until all four samples were individually crushed.

Then, the four samples were combined using a rotary sample splitter (ASTM B21515), shown in Figure 2-4. Each individual crushed sample was poured in the hopper on top of the rotary sample splitter, and a snorkel ventilation system was used for dust control. The sample traveled through the hopper, down a vibratory conveyer belt, and was deposited into one of 12 bins on a rotating platform. After one sample was divided into the 12 bins, the remaining three samples were also run through the rotary sample splitter in the same way, one at a time. This process yielded 12 batches, each batch containing the same amount of each of the four parent samples. At this point, the 12 batches were uniform in composition, but were not mixed. 
Lastly, the 12 individual batches produced from the rotary sample splitter were run through a mechanical splitter (ASTM C702-11) five times. The first four times through the mechanical splitter, the splitter was used to mix the four individual tailings samples into a homogenous sample; after a sample passed through the mechanical splitter, the split sample was re-combined and passed through the splitter again for a total of four passes. The fifth and final time a sample was passed through the mechanical splitter, the splitter was to split each homogenous batch into a smaller sample size, and the resulting sub-samples were kept separate. The final result of this process was 24 homogenous, blended samples, and the properties of this blended batch of tailings are shown in Figure 2-3. 


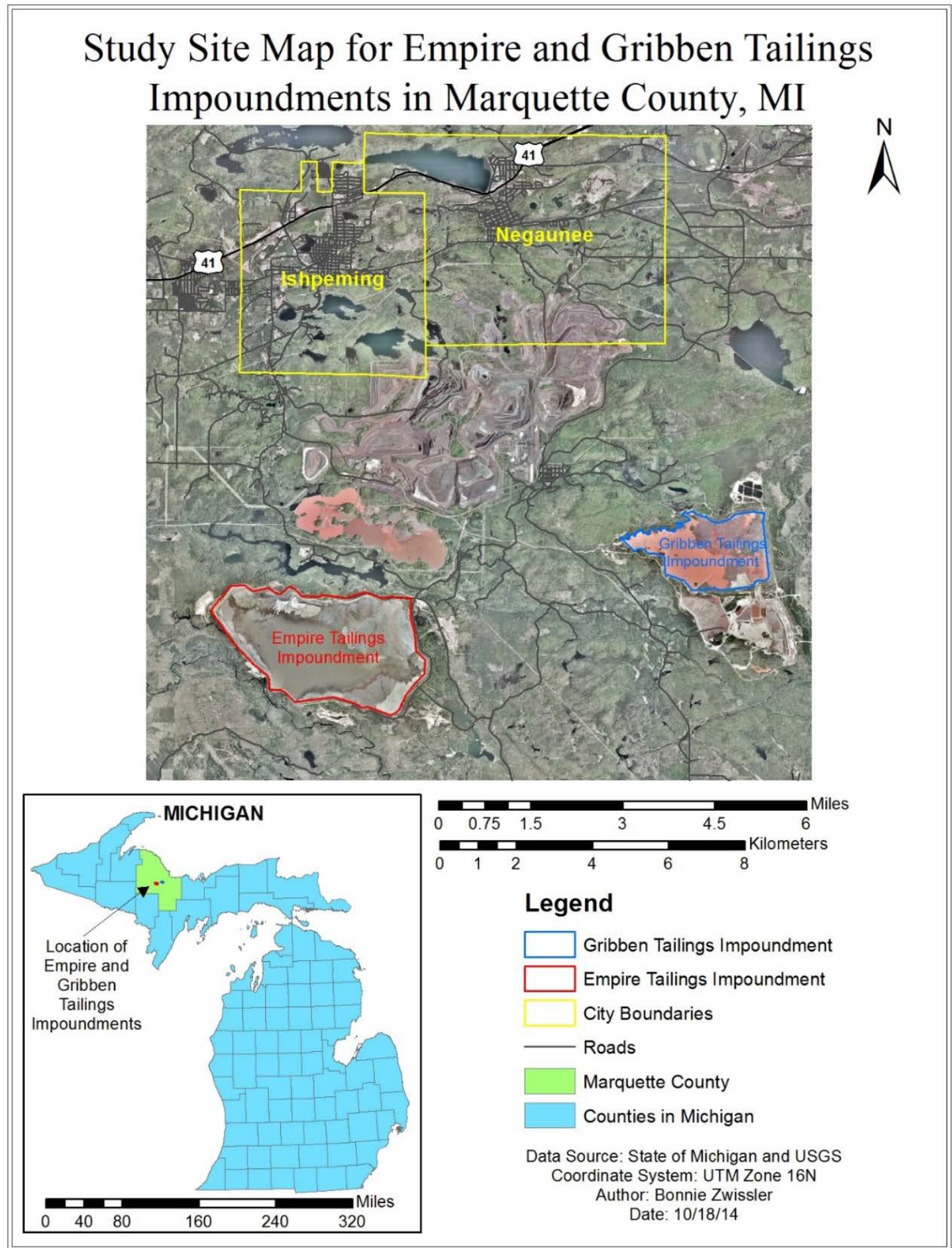

Figure 2-1 | Study site map for Empire and Gribben Mine Tailings Impoundments in Marquette County, MI. 


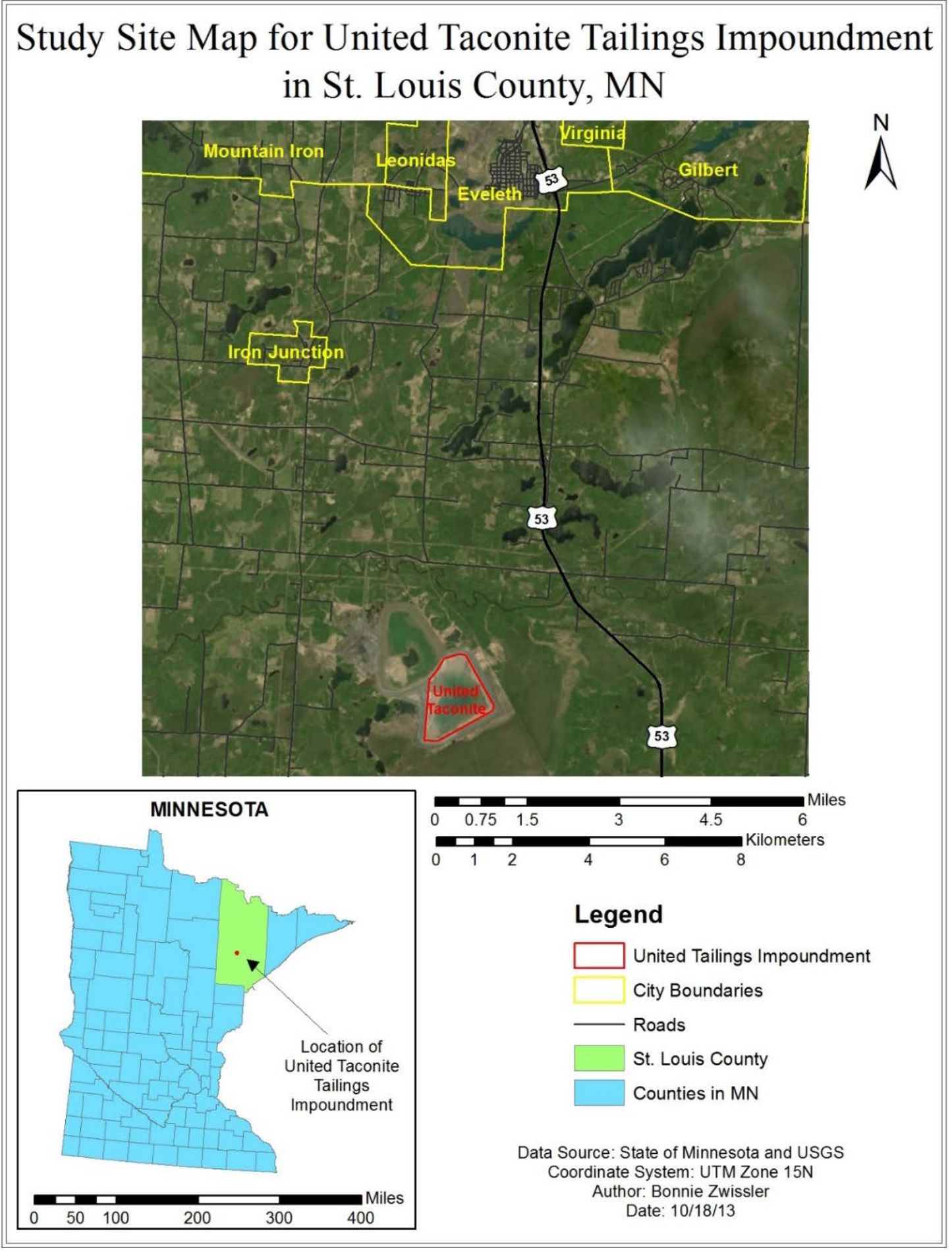

Figure 2-2 | Study site map for United Taconite Mine Tailings Impoundments in St. Louis County, MN. 
Table 2-1 | Geotechnical characterization of mine tailings samples from $M I-m a g, M I$ hem, and MN-mag impoundments.

\begin{tabular}{|c|c|c|c|c|c|c|c|c|c|c|c|}
\hline \multirow{3}{*}{ Sample } & \multirow{3}{*}{$\begin{array}{c}\text { Sample } \\
\text { Date }\end{array}$} & \multicolumn{3}{|c|}{ Specific Gravity* } & \multicolumn{7}{|c|}{ Grain Size Distribution** } \\
\hline & & \multirow[b]{2}{*}{ Mean } & \multirow{2}{*}{$\begin{array}{l}\text { Std. } \\
\text { Dev. }\end{array}$} & \multirow[b]{2}{*}{$\mathrm{n}$} & \multicolumn{2}{|c|}{$\%$ Sand } & \multicolumn{2}{|c|}{$\%$ Silt } & \multicolumn{2}{|c|}{$\%$ Clay } & \multirow[b]{2}{*}{$\mathrm{n}$} \\
\hline & & & & & Mean & $\begin{array}{l}\text { Std. } \\
\text { Dev. }\end{array}$ & Mean & $\begin{array}{l}\text { Std. } \\
\text { Dev. }\end{array}$ & Mean & $\begin{array}{l}\text { Std. } \\
\text { Dev. }\end{array}$ & \\
\hline \multirow{2}{*}{$\begin{array}{l}\text { MI- } \\
\text { mag }\end{array}$} & $\begin{array}{c}\text { October } \\
2012\end{array}$ & 3.086 & 0.011 & 1 & 0.0 & - & 94.7 & - & 5.3 & - & 1 \\
\hline & $\begin{array}{l}\text { July } \\
2013 \\
\end{array}$ & 2.868 & 0.105 & 12 & 41.5 & 41.5 & 56.8 & 43.7 & 1.7 & 2.0 & 12 \\
\hline \multirow{2}{*}{$\begin{array}{l}\text { MI- } \\
\text { hem }\end{array}$} & $\begin{array}{c}\text { October } \\
2012\end{array}$ & 2.942 & 0.009 & 2 & 0.0 & - & 95.5 & - & 4.5 & - & 1 \\
\hline & $\begin{array}{c}\text { July } \\
2013 \\
\end{array}$ & 2.955 & 0.157 & 8 & 7.7 & 3.1 & 88.5 & 3.8 & 3.8 & 3.5 & 8 \\
\hline $\begin{array}{l}\text { MN- } \\
\text { mag }\end{array}$ & $\begin{array}{c}\text { October } \\
2014\end{array}$ & 2.942 & 0.041 & 7 & 39.0 & 26.8 & 58.4 & 24.8 & 3.8 & 2.5 & 7 \\
\hline
\end{tabular}

* Testing performed with Micromeritics AccuPyc 1330 Helium Pycnometer

** Testing performed with hydrometer following ASTM D422-63 (2007) 


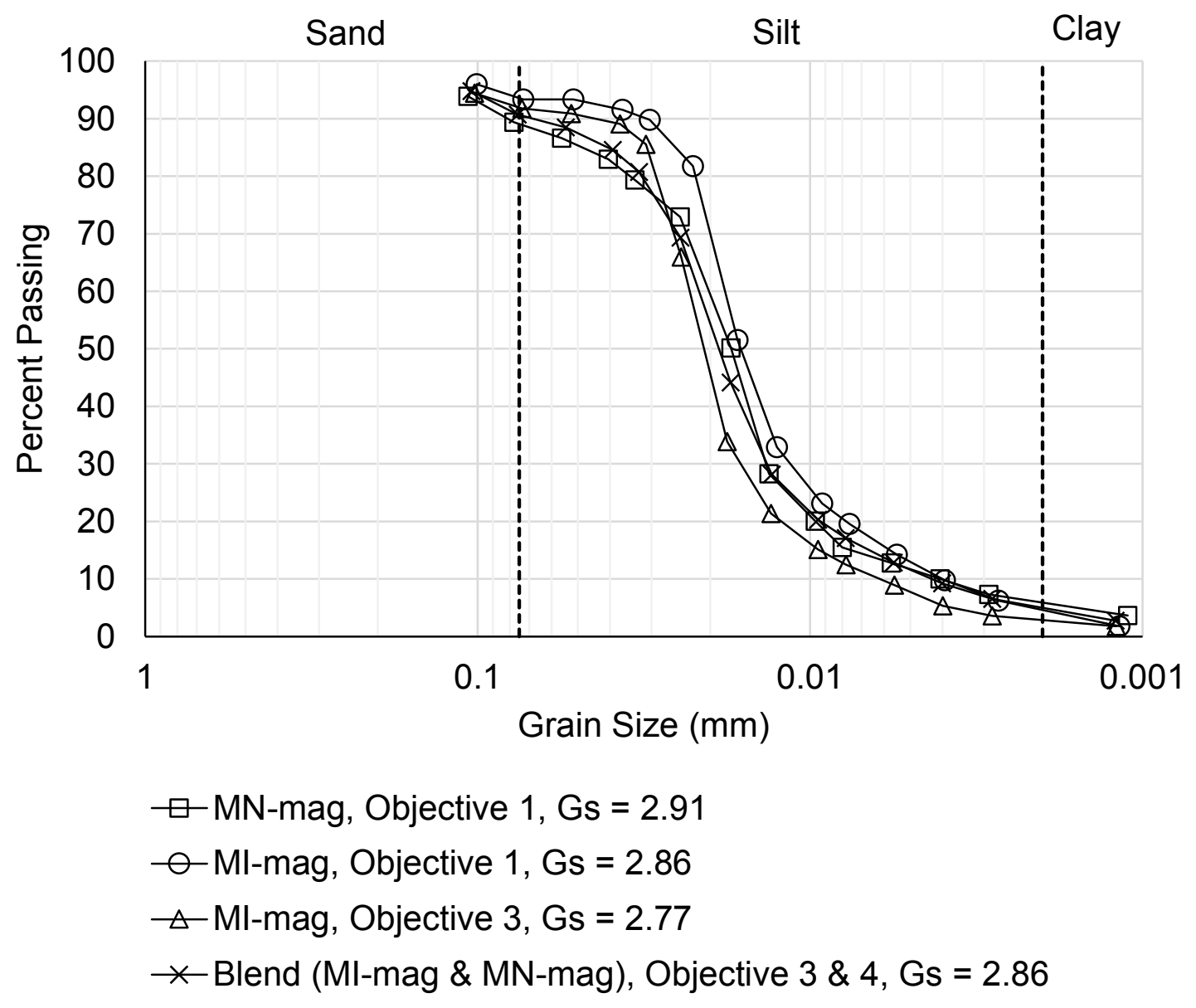

Figure 2-3 | Grain size distributions for MI-mag and MN-mag samples used in laboratory testing. 

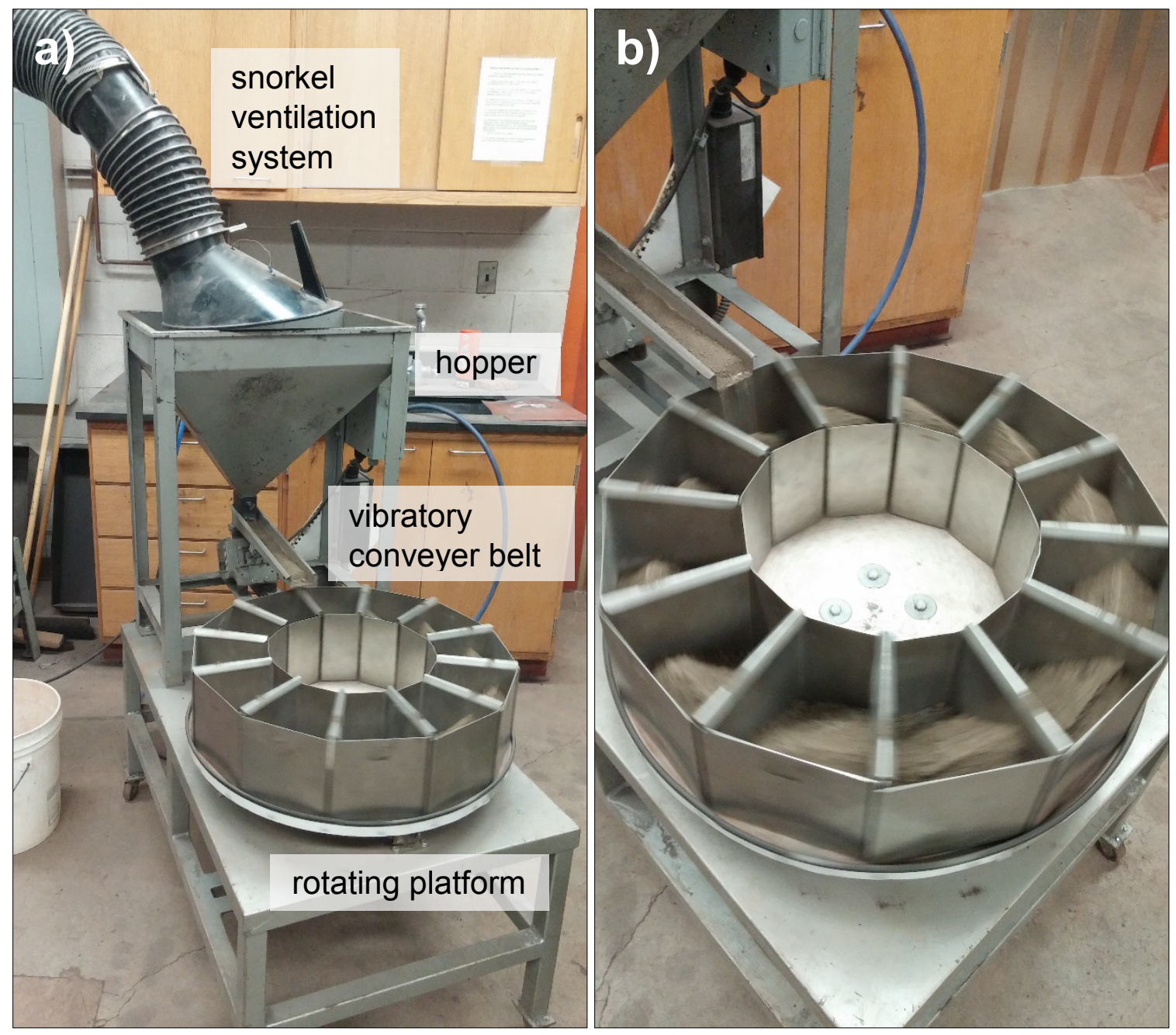

Figure 2-4 | Photo showing rotary sample splitter used for blending of MI-mag and MN-mag samples used in Objectives 3 and 4: (a) rotary sample splitter, showing use of snorkel ventilation system, hopper, vibratory conveyer belt, and twelve sample bins on rotating platform; (b) closer view of vibratory conveyer belt and rotating platform, showing sample being divided into twelve equal parts. 


\subsubsection{Preparation of Laboratory Specimens}

The goal for laboratory sample preparation was to produce samples that mimic the actual conditions in the tailings impoundment as much as possible. All tailings at the impoundments being considered for study are deposited into the impoundments in a 55\% solid/45\% liquid slurry \{Muszynski, 2000 \#128;Price, 1998 \#80;Vasher, 1999 \#79\}, and gradually dry from fully saturated conditions. In the case of the MI-mag tailings, in-situ moisture contents have been measured from 5\% to 30\% by mass (ASTM D2216-10), and void ratios have been measured between 0.7 and 1.2, with a mean of 0.9 (Price 1998). These values were assumed to be representative of the conditions at the MN-mag impoundment as well.

Therefore, all quantitative laboratory testing, which has been conducted on Mi-mag and Mn-mag tailings, was conducted on samples prepared in the same manor. This technique, described in more detail by Buikema (2015), started with the samples fully saturated and with void ratios between 0.8 and 1.0. To achieve these conditions, the wet tailings were air dried for at least 2-3 weeks, and then large soil aggregates that form in the drying process were broken down or "crushed" into individual tailings particles with a Micro-Deval apparatus (ASTM D7428-15). The Micro-Deval cylinder was filled approximately halfway with air-dried tailings, 10 steel ball bearings were added, and the device was run for 10 minutes. After crushing, the tailings were emptied and hand mixed under a snorkel ventilation system with distilled water to achieve a moisture content of $5 \%$ by mass, which was found through trial and error to provide enough moisture so that dust is suppressed, allowing the tailings to be worked without the need for a ventilation system.

Then, the moistened tailings were packed into polycarbonate soil boxes, also referred to in this dissertation as bioreactors. The soil boxes, with inside dimensions of $75 \mathrm{~mm} \mathrm{x}$ $75 \mathrm{~mm} \times 75 \mathrm{~mm}$, were constructed using $6.35 \mathrm{~mm}$ polycarbonate that was adhered using acrylic cement (SCIGRIP 16). Brass or plastic hose barbs $(6.35 \mathrm{~mm})$ were threaded into the bottom of each soil box for saturation and drainage. Before use, the soil boxes were disinfected with $95 \%$ ethanol. To prevent tailings from leaking out of the bottom of the 
soil box via the hose barb and to help facilitate capillary rise, Whatman 41 filter paper (Cat. No. 1441-150) was cut to $75 \mathrm{~mm} \times 75 \mathrm{~mm}$ and placed in the bottom of the soil box, and then a thin layer $(<1 \mathrm{~mm})$ of well-graded sand was placed on top. Subsequently, the soil boxes were packed with mine tailings via pluviation that was achieved using a flour sifter attached to a funnel and hose that allowed the tailings to be distributed evenly. The bottom of the hose was kept within $2 \mathrm{~cm}$ of the soil surface, and approximately $25 \mathrm{~mm}$ of tailings were deposited in the bottom of the soil box and compacted using a large hand tamp (50 mm diameter), followed by a small hand tamp (19 $\mathrm{mm}$ diameter). This process was repeated for seven to ten lifts until the soil box was full of compacted tailings. The soil box packing technique is summarized in the schematic presented in Figure 2-5. After being filled, void ratio was calculated for each soil box, and any sample that did not fall within the acceptable void ratio range was discarded and re-packed. Lastly, polypropylene tubing was connected to the hose barb to saturate the soil boxes with sterile distilled water using a Mariotte tube constant head device, as illustrated by the schematic in Figure 2-6.

Preparation of all laboratory specimens in accordance with these methods, in a repeatable way and to moisture/density conditions representative of the tailings impoundments, allowed for results to be compared within and between testing for all objectives. 


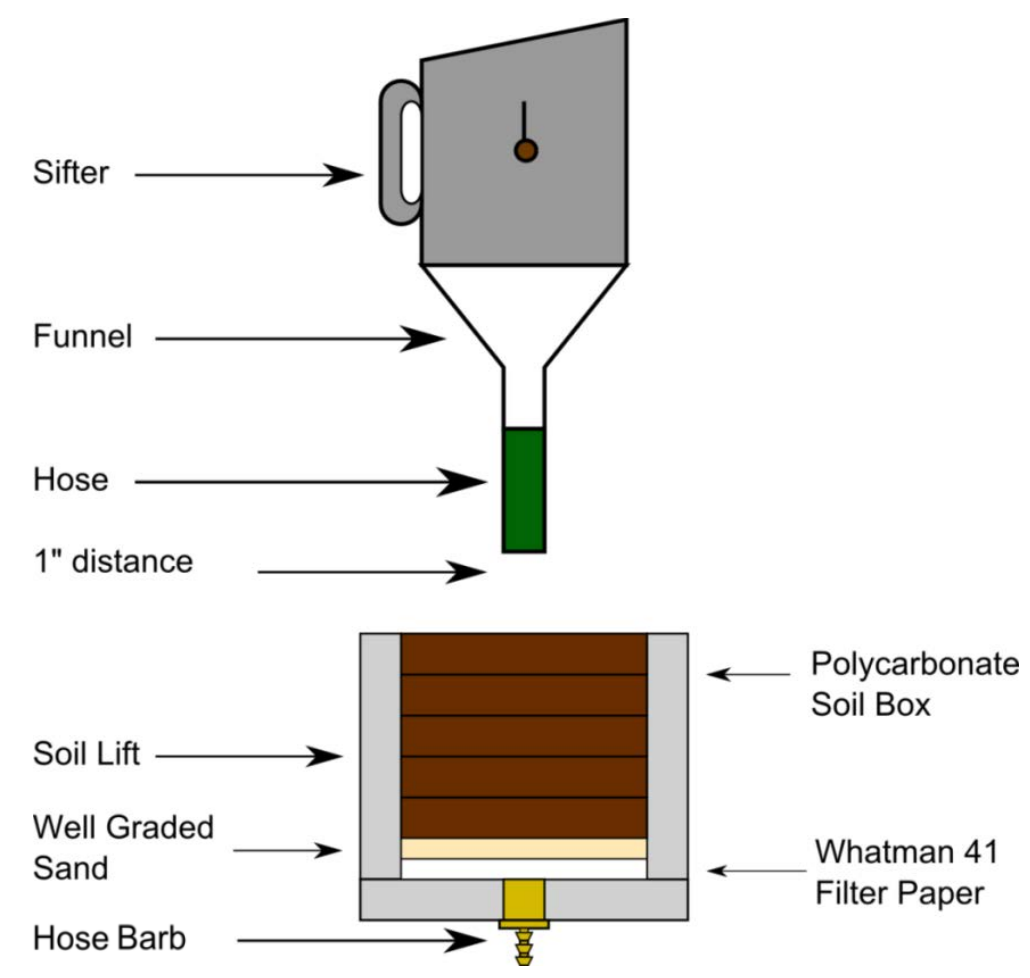

Figure 2-5 | Schematic of soil box packing technique, used in the preparation of all laboratory samples.

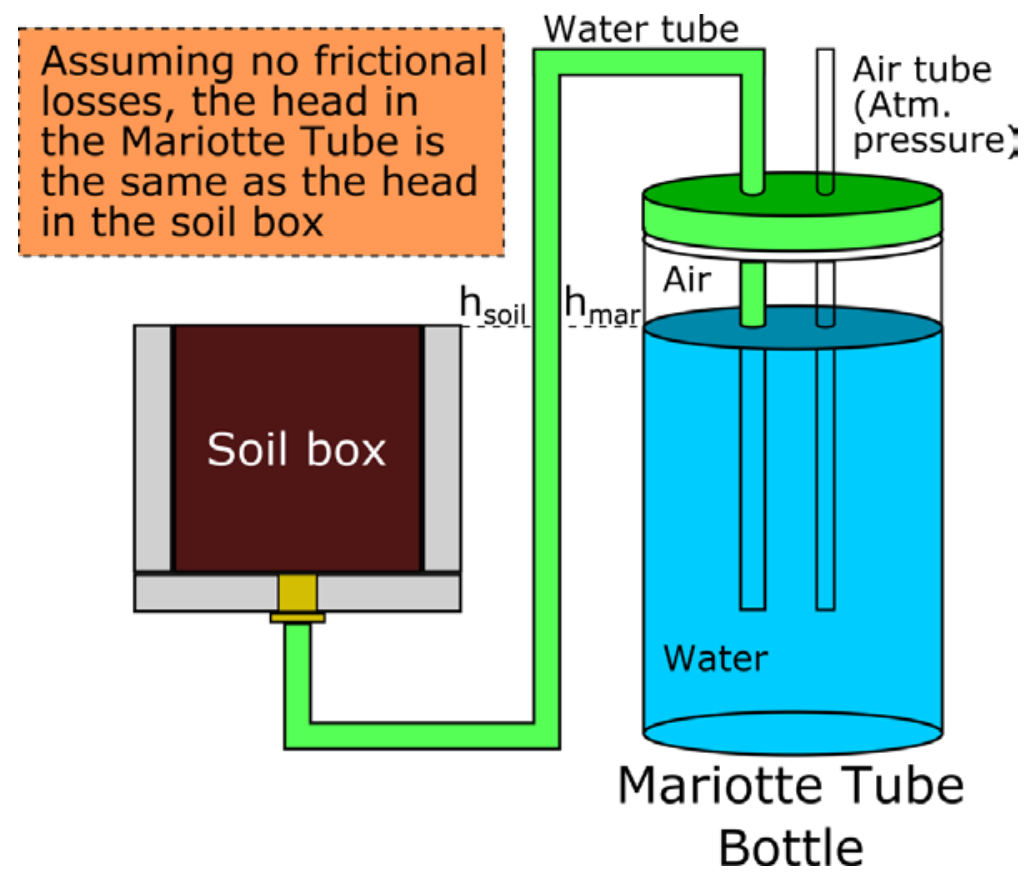

Figure 2-6 | Schematic of Mariotte Tube, used for saturation of tailings samples. 


\subsection{Methods to Satisfy Objective 1}

To satisfy Objective 1, a quantitative relationship between thermal remote sensing, moisture content, and surface strength for both MI-mag and MN-mag tailings was analyzed.

\subsubsection{Laboratory testing of thermal properties, moisture content, and strength}

Samples were prepared as described in Section 2.1.2 to consistent density and moisture conditions. Subsequently, samples starting at fully saturated conditions were exposed to an artificial diurnal heating cycle simulated with halogen and full-spectrum lights and were monitored and tested until the samples were dry. This approach provided a range of moisture/strength conditions per sample over the duration of testing.

Diurnal heating cycles were simulated in the laboratory by heating samples for 6 hours daily under two 500-Watt halogen lamps and two 85 Watt/300 Watt-equivalent full spectrum lights mounted at a $45^{\circ}$ angle to the samples at a height of $0.7 \mathrm{~m}$ above the samples, as shown in Figure 2-7. Thermal imaging and strength measurements were taken twice daily for samples - once before heating and once after heating. These measurements are outlined in Table 2-2, and are described in more detail below.

Moisture content (\% by mass) was determined by monitoring sample mass loss on a balance. The sample mass measured at a given sampling event was subtracted from the initial sample mass recorded during sample preparation to determine the mass of water lost, which was used to back-calculate moisture content. The reported moisture content is the average moisture content for the entire $76 \mathrm{~mm} \times 76 \mathrm{~mm} \times 76 \mathrm{~mm}$ sample, and it is worth noting that the surface moisture content conditions are likely lower than the bulk moisture content. However, because the samples started at the same conditions and were exposed to the same heating conditions, it is expected that all samples dried in the same way, and therefore the bulk moisture content should have a direct relationship with surface moisture content. For ease of data collection, bulk moisture content was measured rather than surface moisture content. 
Relative soil strength was quantified using the penetration depth $(\mathrm{cm})$ of a Humboldt H-1200 universal needle penetrometer with Humboldt H-1280 40-45 mm standard hardened stainless steel needle, $150 \mathrm{~g}$ drop weight, and drop duration of 0.5 seconds. Five measurements were taken at each sampling event, one in each quadrant of the soil box and one in the center, with no less than $3 \mathrm{~cm}$ between penetration positions. The measurements were then averaged to yield an average penetration depth, which was used to represent the relative surface strength of each sample.

Atmospheric temperature $(\mathrm{K})$ and relative humidity (\%) were measured using a thermohygrometer pre-heating and post-heating.

Surface albedo was measured using an ASD FieldSpec $®$ HandHeld Pro (325$1075 \mathrm{~nm}$ ) with plant probe; the average of 10 spectral reflectance curves per sample was used to calculate albedo as the mean reflectance from 400 to $1075 \mathrm{~nm}$.

Surface temperature (K) was measured using a FLIR ThermaCAM SC640 thermal camera. One thermal image was captured per sample both before the artificial diurnal heating cycle began (immediately before lights were turned on) and at the end of the diurnal heating cycle (immediately before lights were turned off). The images were taken from the same location each time, $0.7 \mathrm{~m}$ above the sample surface. For the data collected using the thermal camera to be converted to represent surface temperature rather than radiant energy (the raw data collected), FLIR object parameters were measured and used each time an image was taken, as described in Table 2-3. The surface temperature of each sample, which was used to calculate the change in surface temperature, was determined using FLIR ThermaCAM Researcher software and these object parameters, as shown in Figure 2-8. For each sample, the surface temperature for each image was determined; the average sample temperature from pre-heating images was used to represent pre-heating surface temperature, and the average sample temperature from post-heating images was used to represent post-heating surface temperature. The difference between pre-heating and post-heating surface temperature represented change in surface temperature $(\Delta \mathrm{T})$. The magnitude of soil temperature change, with respect to atmospheric temperature change, is a function of soil moisture. This is why the study looked at change in 
temperature due to diurnal heating cycles, rather than just an average daily temperature or an instantaneous temperature.

Testing of the MI-mag tailings occurred during the summer of 2014, while testing of the MN-mag tailings occurred in the summer of 2015. Between two and four samples were tested at a time, with one data point collected per day per sample, yielding 45 total data points for the MI-mag tailings and 48 total data points for the MN-mag tailings. After all data were collected, the relationships between the measured variables for each set of tailings were explored using regression analysis.

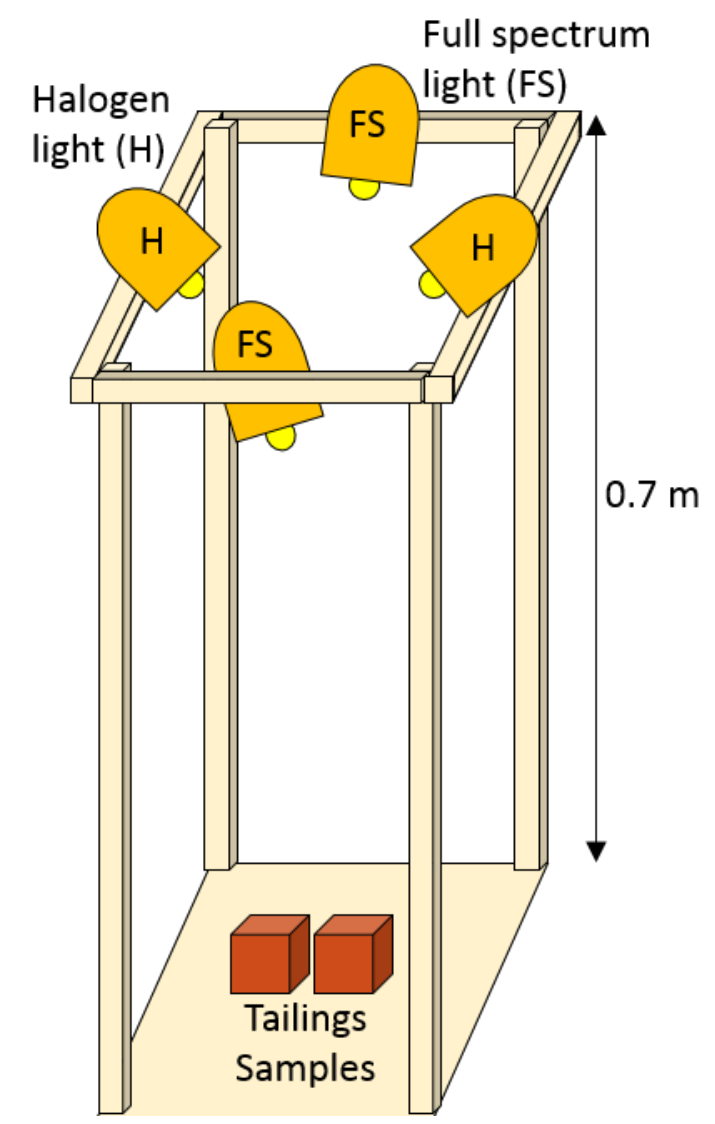

Figure 2-7 | Schematic of laboratory thermal remote sensing testing set-up. 
Table 2-2 | Details of thermal remote sensing laboratory testing conducted per sampling event.

\begin{tabular}{|l|l|l|l|}
\hline $\begin{array}{l}\text { Measurement } \\
\text { Collected }\end{array}$ & Instrumentation & Purpose of Measurement & $\begin{array}{l}\text { Tests } \\
\text { per } \\
\text { Sample }\end{array}$ \\
\hline Mass & AND EK Balance & $\begin{array}{l}\text { Moisture content } \\
\text { calculation }\end{array}$ & 1 \\
\hline Penetration depth & $\begin{array}{l}\text { Humboldt H-1200 } \\
\text { needle penetrometer }\end{array}$ & $\begin{array}{l}\text { Represent relative surface } \\
\text { strength }\end{array}$ & 5 \\
\hline $\begin{array}{l}\text { Atm. } \\
\text { temp./humidity }\end{array}$ & $\begin{array}{l}\text { General Tools PTH8708 } \\
\text { Thermohygrometer }\end{array}$ & $\begin{array}{l}\text { Quantify atmospheric } \\
\text { variation }\end{array}$ & 1 \\
\hline Spectral reflectance & $\begin{array}{l}\text { FieldSpec HandHeld } \\
\text { Pro Spectroradiometer } \\
\text { with plant probe }\end{array}$ & Albedo calculation (ATI) & 10 \\
\hline Thermal image & $\begin{array}{l}\text { FLIR SC640 thermal } \\
\text { camera }\end{array}$ & $\begin{array}{l}\text { Quantify } \Delta \text { T due to diurnal } \\
\text { heating (ATI) }\end{array}$ & 1 \\
\hline
\end{tabular}

Table 2-3 | Description of each FLIR object parameter and how the parameter was quantified.

\begin{tabular}{|l|l|}
\hline Object Parameter & How to Quantify Each Parameter \\
\hline Emissivity & $\begin{array}{l}\text { Calculated as complement of albedo from } \\
\text { spectroradiometer }\end{array}$ \\
\hline Reflected apparent temperature (K) & Same as atmospheric temperature \\
\hline Object distance (m) & $\begin{array}{l}0.7 \text { meters (dist. between sample surface and } \\
\text { camera lens) }\end{array}$ \\
\hline Atmospheric temperature (K) & $\begin{array}{l}\text { Measured with thermohygrometer between sample } \\
\text { and lens }\end{array}$ \\
\hline Relative humidity (\%) & $\begin{array}{l}\text { Measured with thermohygrometer between sample } \\
\text { and lens }\end{array}$ \\
\hline $\begin{array}{l}\text { External optics temperature (K) and } \\
\text { transmission }\end{array}$ & 1.0 (value used when no external optics used) \\
\hline
\end{tabular}



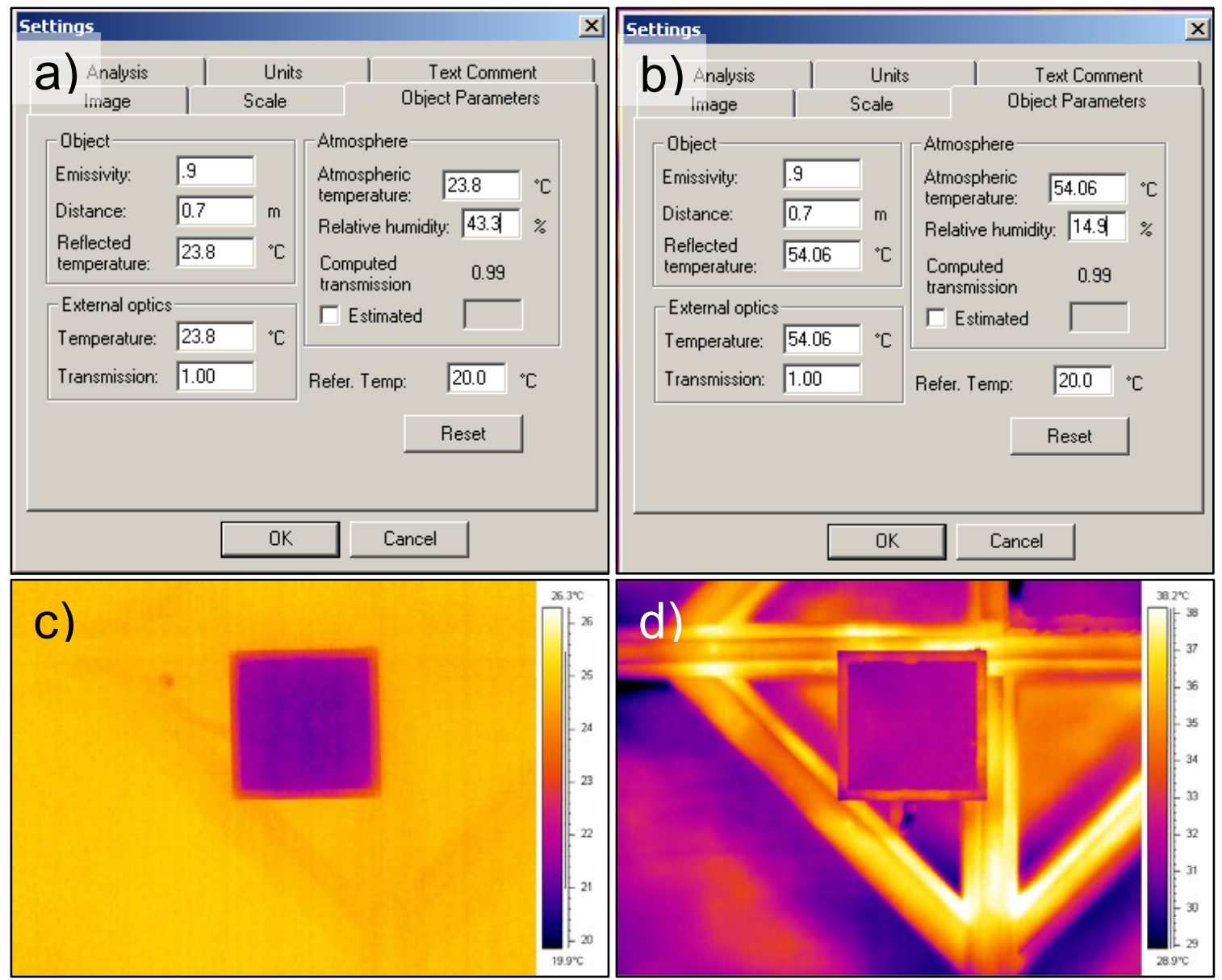

Figure 2-8 | Sample temperature determination using FLIR ThermaCam

Researcher Professional software using thermal images for MN-Mag sample tested on June 4, 2015: (a) object parameter entry for pre-heating conditions, (b) object parameter entry for post-heating conditions, (c) thermal image representing surface temperature for pre-heating sample, and (d) thermal image representing surface temperature for postheating sample. Using the pre- and post- heating thermal images (c and d), the average surface temperature for the square sample was determined and used to calculate change in surface temperature (average pre-heating sample temperature from (c) of $21.4^{\circ} \mathrm{C}$ or $294.6 \mathrm{~K}$, average post-heating sample temperature from $(\mathrm{d})$ of $31.8^{\circ} \mathrm{C}$ or $305.0 \mathrm{~K}, \Delta \mathrm{T}$ of $10.4 \mathrm{~K})$. 


\subsection{Methods to Satisfy Objective 2}

To satisfy Objective 2, the application of relationship developed in Objective 1 for MI-mag and $M N$-mag tailings was tested in the field.

\subsubsection{Collection of Thermal Images and Ground Truth Data}

For proof-of-concept testing of the different methods used to apply the laboratory relationship(s) developed in Objective 1 to field scale, numerous field campaigns were carried out. Details of the dates, locations, and purposes of these field visits are provided in Table 2-4, and more specifics of the field activities carried out are described below.

Table 2-4 | Details of site visits to MI-mag, MI-hem, and MN-mag impoundments.

\begin{tabular}{|l|l|l|}
\hline Date & Impoundment(s) & Purpose \\
\hline Oct 2012 & MI-mag, MI-hem & $\begin{array}{l}\text { View impoundments, collect samples for } \\
\text { laboratory geotechnical characterization }\end{array}$ \\
\hline July 2013 & MI-mag, MI-hem & $\begin{array}{l}\text { Collect samples for laboratory geotechnical } \\
\text { characterization + laboratory sample } \\
\text { preparation }\end{array}$ \\
\hline Oct 2014 & MN-mag & $\begin{array}{l}\text { View impoundment, collect samples for } \\
\text { laboratory geotechnical characterization, } \\
\text { perform ground truthing (moisture content, } \\
\text { strength, density) to pair with satellite } \\
\text { overpass* }\end{array}$ \\
\hline May 2015 & MN-mag & $\begin{array}{l}\text { Perform ground truthing (moisture content) to } \\
\text { pair with satellite overpass* and collect UAV- } \\
\text { based thermal images }\end{array}$ \\
\hline April 2016 & MN-mag & $\begin{array}{l}\text { Perform ground truthing (moisture content, } \\
\text { strength) and collect hand-held thermal } \\
\text { images** }\end{array}$ \\
\hline June 2016 & MI-mag & $\begin{array}{l}\text { Perform ground truthing (moisture content, } \\
\text { strength) and collect hand-held thermal images }\end{array}$ \\
\hline
\end{tabular}

*Satellite image acquisition to be paired with this field testing was unsuccessful ** Impoundment was unexpectedly frozen, so sample collection and strength measurements were unable to be made; impoundment was not actively discharging, so all areas had been covered with hay mulch and thermal imaging would not have been of bare tailings.

For the October 2012 and July 2013 field visits, samples were collected using shovels and buckets and transported to the lab for geotechnical characterization. The methods used for this geotechnical characterization are discussed in Section 2.1.1. Once it became 
clear what additional field data would be useful to collect, the testing conducted during site visits expanded in scope. And as more field campaigns were performed and methods were refined based on what data was needed to satisfy Objective 2, the ground truth tests (used to calibrate laboratory data and thermal imagery with actual field conditions) and method of thermal image acquisition were modified. Table 2-5 outlines the data that were collected in Oct 2014, Table 2-6 outlines the data that were collected in May 2015, and Table 2-7 outlines the data that were collected in June 2016. Ultimately, the June 2016 data were used to assess the application of laboratory relationship(s) developed in Objective 1 to field scale, as discussed in Chapter 4. Additional details about the field activities are described below.

Table 2-5 | Details of field testing in Oct 2014 for geotechnical characterization and ground truthing to validate satellite data. Seven sites around the northern half of the MNMag impoundment were studied.

\begin{tabular}{|l|l|l|l|}
\hline $\begin{array}{l}\text { Data } \\
\text { Collected }\end{array}$ & Equipment Used & Purpose & Tests per Site \\
\hline $\begin{array}{l}\text { Location } \\
\text { details }\end{array}$ & GPS & $\begin{array}{l}\text { To be able to relate tailings } \\
\text { data to satellite data }\end{array}$ & 1 \\
\hline $\begin{array}{l}\text { Atm. temp. } \\
\text { \& humidity }\end{array}$ & Thermohygrometer & $\begin{array}{l}\text { To quantify atm. conditions } \\
\text { across impoundment }\end{array}$ & 1 \\
\hline $\begin{array}{l}\text { Tailings } \\
\text { samples }\end{array}$ & $\begin{array}{l}\text { Shovel \& } \\
\text { bucket/bag }\end{array}$ & $\begin{array}{l}\text { For tailings characterization } \\
\text { in lab (grain size }+ \text { Gs) }\end{array}$ & $\begin{array}{l}1 \text { sample (for } 2 \\
\text { Gs \& } 2 \text { grain } \\
\text { size per sample) }\end{array}$ \\
\hline $\begin{array}{l}\text { Moisture \& } \\
\text { density }\end{array}$ & $\begin{array}{l}\text { Nuclear density } \\
\text { gauge }\end{array}$ & $\begin{array}{l}\text { To quantify moisture and } \\
\text { density variation across } \\
\text { impoundment }\end{array}$ & 3 \\
\hline $\begin{array}{l}\text { Thermal } \\
\text { properties }\end{array}$ & Thermal probe & $\begin{array}{l}\text { To relate to laboratory } \\
\text { thermal data }\end{array}$ & 2 \\
\hline Albedo & $\begin{array}{l}\text { Spectroradiometer } \\
+ \text { plant probe }\end{array}$ & $\begin{array}{l}\text { To calculate apparent } \\
\text { thermal inertia }\end{array}$ & $\begin{array}{l}3 \text { locations per } \\
\text { site }\end{array}$ \\
\hline Strength & Vane shear, CPT & $\begin{array}{l}\text { To quantify surface strength } \\
\text { of tailings }\end{array}$ & $\begin{array}{l}5-10 \text { of each test } \\
\text { per location }\end{array}$ \\
\hline $\begin{array}{l}\text { Handheld } \\
\text { thermal } \\
\text { images of } \\
\text { tailings }\end{array}$ & $\begin{array}{l}\text { FLIR SC } 640 \\
\text { camera }\end{array}$ & $\begin{array}{l}\text { To quantify surface } \\
\text { temperature variation \& to } \\
\text { calculate } \Delta T\end{array}$ & $\begin{array}{l}\text { To quantify surface } \\
\text { temperature variation \& to } \\
\text { calculate } \Delta T\end{array}$ \\
\hline $\begin{array}{l}\text { Satellite } \\
\text { thermal } \\
\text { images }\end{array}$ & ASTER & 1 image pair \\
\hline
\end{tabular}


Table 2-6 | Details of field testing in May 2015 for geotechnical characterization and ground truthing to validate satellite data. Thirty sites around the south-eastern region of the MN-Mag impoundment were studied.

\begin{tabular}{|l|l|l|l|}
\hline $\begin{array}{l}\text { Data } \\
\text { Collected }\end{array}$ & Equipment Used & Purpose & Tests per Site \\
\hline $\begin{array}{l}\text { Location } \\
\text { details }\end{array}$ & GPS & $\begin{array}{l}\text { To be able to relate tailings } \\
\text { data to satellite/UAV data }\end{array}$ & 1 \\
\hline $\begin{array}{l}\text { Atm. temp. } \\
\text { \& humidity }\end{array}$ & Thermohygrometer & $\begin{array}{l}\text { To quantify atm. conditions } \\
\text { across impoundment }\end{array}$ & 1 \\
\hline $\begin{array}{l}\text { Tailings } \\
\text { samples }\end{array}$ & Spoon \& bag & $\begin{array}{l}\text { For moisture content } \\
\text { characterization in lab }\end{array}$ & 1 sample \\
\hline $\begin{array}{l}\text { Thermal } \\
\text { images of } \\
\text { tailings }\end{array}$ & $\begin{array}{l}\text { FLIR SC 640 } \\
\text { camera }\end{array}$ & $\begin{array}{l}\text { To quantify surface } \\
\text { temperature variation \& to } \\
\text { calculate ATI }\end{array}$ & 5 images \\
\hline
\end{tabular}

Table 2-7 | Details of field testing in June 2016 for geotechnical characterization and ground truthing to validate satellite data. Fifteen sites on the eastern side of the MI-Mag impoundment were studied, and measurements were taken pre- and post-heating due to a 6 hour diurnal heating cycle.

\begin{tabular}{|l|l|l|l|}
\hline $\begin{array}{l}\text { Data } \\
\text { Collected }\end{array}$ & Equipment Used & Purpose & Tests per Site \\
\hline $\begin{array}{l}\text { Location } \\
\text { details }\end{array}$ & GPS & $\begin{array}{l}\text { To be able to relate tailings } \\
\text { data to satellite/UAV data }\end{array}$ & 1 \\
\hline $\begin{array}{l}\text { Atm. temp. } \\
\text { \& humidity }\end{array}$ & Thermohygrometer & $\begin{array}{l}\text { To quantify atm. conditions } \\
\text { across impoundment }\end{array}$ & $\begin{array}{l}1 \text { measurement, } \\
\text { pre/post heating }\end{array}$ \\
\hline $\begin{array}{l}\text { Tailings } \\
\text { samples }\end{array}$ & Spoon \& bag & $\begin{array}{l}\text { For moisture content } \\
\text { characterization in lab }\end{array}$ & $\begin{array}{l}1 \text { sample, } \\
\text { pre/post heating }\end{array}$ \\
\hline $\begin{array}{l}\text { Tailings } \\
\text { strength }\end{array}$ & Vane shear & $\begin{array}{l}\text { To quantify surface strength } \\
\text { of tailings }\end{array}$ & $\begin{array}{l}5 \text { per location, } \\
\text { pre/post heating }\end{array}$ \\
\hline $\begin{array}{l}\text { Thermal } \\
\text { images of } \\
\text { tailings }\end{array}$ & $\begin{array}{l}\text { FLIR SC 640 } \\
\text { camera }\end{array}$ & $\begin{array}{l}\text { To quantify surface } \\
\text { temperature variation \& to } \\
\text { calculate ATI }\end{array}$ & $\begin{array}{l}5 \text { images, } \\
\text { pre/post heating }\end{array}$ \\
\hline
\end{tabular}

Location details were measured using a handheld GPS unit, and was used spatial visualization of the sampling sites.

Atmospheric temperature $(\mathrm{K})$ and relative humidity (\%) were measured using a thermohygrometer at each location during both pre- and post-diurnal heating. 
Tailings samples were collected using either a shovel or spoon and placed into a bucket or bag for transport back to the laboratory. Care was taken when collecting tailings to sample only the surface material (top 5-10 cm).

Gravimetric moisture content (\% by mass) was determined by for each sample collected following ASTM D2216-10. The daily average moisture content for each site was used in data analysis.

Tailings in-situ moisture content and density was determined using a nuclear density gage. Measurements were taken at a depth of $15 \mathrm{~cm}$ following ASTM D6938-15.

Tailings thermal properties were measured using a thermal probe following ASTM D5334-14. The KD2 Pro SH-1 dual-needle thermal diffusivity and specific heat sensor was used to measure the thermal properties in the upper $5 \mathrm{~cm}$ of the tailings. Per manufacturer guidelines, 15 minutes was allowed between readings to allow the probe and soil to re-equilibrate.

Surface albedo was measured using an ASD FieldSpec $®$ HandHeld Pro (325$1075 \mathrm{~nm}$ ) with plant probe; the average of 10 spectral reflectance curves per test was used to calculate albedo as the mean reflectance from 400 to $1075 \mathrm{~nm}$, because readings below $400 \mathrm{~nm}$ contained excessive noise.

Relative soil strength was quantified using either a handheld CPT device or a handheld vane shear device. The CPT measured cone tip resistance with depth, up to a depth of $45.7 \mathrm{~cm}$. For data analysis, the average cone tip resistance in the upper $15 \mathrm{~cm}$ was used. The vane shear measured degrees of rotation, which was converted to shear strength using ASTM 4648-16. All vane shear measurements were made at the tailings surface. For all strength tests, at least 5 measurements were taken per sample, and the average (daily) value was used to represent tailings strength.

Surface temperature (K) was measured using a FLIR ThermaCAM SC640 thermal camera. Five thermal image was captured per study site, both at the start and end of the diurnal heating cycle. The images were taken from the same location each time, $1.2 \mathrm{~m}$ 
above the sample surface. As discussed in Section 2.1.1, for the image collected using the thermal camera to be converted to represent surface temperature rather than radiant energy (the raw data collected), FLIR object parameters were measured and used each time an image was taken (Table 2-3). The surface temperature of each study site, which was used to calculate the change in surface temperature, was determined using FLIR ThermaCAM Researcher software and these object parameters (Figure 2-8). For each study site, the surface temperature for each image was determined, the average of the five pre-heating images was used to represent pre-heating surface temperature, and the average of the five post-heating images was used to represent post-heating surface temperature. The difference between pre-heating and post-heating surface temperature represented change in surface temperature $(\Delta \mathrm{T})$.

\subsubsection{Proof-of-Concept Satellite and UAV Imagery Assessment}

The imaging employed in this study was not continuous imagery over an entire impoundment. The methods described in Sections 2.3.1 did satisfy Objective 2, applying the laboratory relationships between thermal remote sensing and moisture content/strength to field scales. However, for real-world application, it would be more useful to collect continuous imagery over an entire impoundment. This would allow the generation of maps to identify spatial and temporal changes in moisture content/strength for tailings impoundments, which could prove to be quite useful to tailings impoundment managers. Developing methods to produce such maps was outside of the scope of this study, but some proof-of concept work on such applications was conducted.

Satellite data is one way that the laboratory relationship between thermal remote sensing and moisture content could be applied to field scale applications and used to produce useful maps. ASTER satellite data has $90 \mathrm{~m}$ spatial resolution, which should be sufficient to demonstrate surface changes across the multiple-square-kilometer tailings impoundments. Another potential solution involves using a UAV to collect thermal images. A comparison between the use of ASTER and UAV thermal imagery is presented in Table 2-8. 
Table 2-8 | Comparison between satellite and UAV thermal remote sensing

\begin{tabular}{|l|l|l|l|l|}
\hline & ASTER pros & ASTER cons & UAV pros & UAV cons \\
\hline $\begin{array}{l}\text { Temporal } \\
\text { resolution }\end{array}$ & $\begin{array}{l}\text { Scheduled } \\
\text { satellite overpass } \\
\text { (16 days) }\end{array}$ & $\begin{array}{l}\text { Requires data } \\
\text { Acquisition } \\
\text { Request, clouds } \\
\text { can interfere }\end{array}$ & $\begin{array}{l}\text { "On demand" } \\
\text { data, can fly } \\
\text { under clouds }\end{array}$ & $\begin{array}{l}\text { Must be present } \\
\text { for data to be } \\
\text { collected }\end{array}$ \\
\hline $\begin{array}{l}\text { Spatial } \\
\text { resolution }\end{array}$ & $\begin{array}{l}\text { Easy to cover } \\
\text { large area in } \\
\text { single image }\end{array}$ & $\begin{array}{l}30 \mathrm{~m} \text { (visible) } \\
90 \mathrm{~m} \text { (thermal) }\end{array}$ & $\begin{array}{l}\text { High (cm scale), } \\
\text { set by flying } \\
\text { height }\end{array}$ & $\begin{array}{l}\text { Can vary if fly } \\
\text { height not } \\
\text { controlled }\end{array}$ \\
\hline $\begin{array}{l}\text { Spectral } \\
\text { resolution }\end{array}$ & $\begin{array}{l}\text { Multiple bands } \\
\text { (3 visible, 5 } \\
\text { thermal) }\end{array}$ & $\begin{array}{l}\text { No band } \\
\text { control, if } \\
\text { sensor dies } \\
\text { that's it }\end{array}$ & $\begin{array}{l}\text { Control over } \\
\text { band(s) } \\
\text { collected }\end{array}$ & $\begin{array}{l}\text { Camera } \\
\text { selection limited } \\
\text { by weight }\end{array}$ \\
\hline Processing & $\begin{array}{l}\text { Straightforward } \\
\text { (DN } \rightarrow\end{array}$ & $\begin{array}{l}\text { Must wait to } \\
\text { reflectance/temp) }\end{array}$ & $\begin{array}{l}\text { Can process as } \\
\text { soon as UAV } \\
\text { lands }\end{array}$ & $\begin{array}{l}\text { Intensive } \\
\text { (mosaic, } \\
\text { georeference, } \\
\text { scale) }\end{array}$ \\
\hline Cost & $\begin{array}{l}\text { Free for } \\
\text { education and } \\
\text { non-education } \\
\text { (as of 4/2016) }\end{array}$ & N/A & $\begin{array}{l}\text { Low once } \\
\text { equipment } \\
\text { owned }\end{array}$ & $\begin{array}{l}\text { Initial } \\
\text { investment for } \\
\text { UAV, camera, } \\
\text { etc }\end{array}$ \\
\hline
\end{tabular}

To assess the feasibility of using satellite data to monitor spatial and temporal changes in the surface conditions of tailings impoundments, ASTER satellite data were used to study the Empire Tailings Impoundment. As discussed in Section 1.3, ASTER collects 14 bands of data, ranging from visible to thermal infrared, with at least $90 \mathrm{~m}$ spatial resolution. ASTER's 14 bands consist of: 3 bands of visible and near infrared (VNIR) with $15 \mathrm{~m}$ spatial resolution; 6 bands of short wave infrared (SWIR) with $30 \mathrm{~m}$ spatial resolution; and 5 bands of thermal infrared (TIR) imaging with $90 \mathrm{~m}$ resolution. Each data scene covers an area of $60 \mathrm{~km}$ x $60 \mathrm{~km}$. ASTER's orbit repeats every 16 days, but non-nadir scenes are available more frequently (Abrams et al. 2002).

For this preliminary assessment, the ability to detect spatial variation in surface temperature was studied. Two scenes of ASTER L1A data were obtained from April and July of 2002 for the Empire Tailings Impoundment. The two images were processed using ERDAS IMAGINE 2013 and ArcMap 10.1 using the procedure outlined in Figure 2-9 to obtain change in surface temperature for the impoundment. 
For change in temperature $(\Delta \mathrm{T})$, the ASTER L1A spectral radiance was converted to brightness temperature (Ghulam 2009) and then to top-of-atmosphere temperature (Yale Center for Earth Observations 2012), as described in Figure 2-9. The change in surface temperature is intended to be calculated for a single diurnal heating cycle (between the daily high and low temperature), but ASTER scenes were not available at that frequency for this study site. Therefore, two scenes from 2002 (one in April and one in July) were selected, as a significant surface temperature change between these two months was expected and is observed, as shown in Figure 2-10. The fact that changes in surface temperature can be detected between any two ASTER scenes means that if data can be collected for a single diurnal heating cycle, changes in surface temperature can also be calculated.

It should also be noted that technically, what has been calculated and used in this proof-of-concept assessment is brightness temperature and not surface temperature. Brightness temperature can be converted to surface temperature with knowledge of the atmospheric conditions at the time of image acquisition. Because the atmospheric conditions were not known (and incorrect assumptions would add more uncertainty), brightness temperature is assumed to be interchangeable for surface temperature. With data on the atmospheric conditions, the brightness temperature values could be converted to surface temperature in future studies.

In cases where satellite imagery does not provide satisfactory results, another potential solution to utilize involves using a UAV to collect thermal images.

To assess the feasibility of this option, a FLIR Tau 2 thermal imaging camera attached to a DJI Phantom quadcopter UAV was flown on May 26, 2015 and used to collect thermal images of the surface of the United Taconite tailings basin. The Tau 2 thermal camera collects a single band of data $(7.5-13.5 \mu \mathrm{m})$ that could easily be calibrated with surface conditions and used to map spatial and temporal changes in thermal properties, moisture content, and strength for the tailings. 
While the UAV-based thermal imagery was not processed to calculate surface temperature, it was qualitatively assessed, and sample images can be seen in Figure 2-11. A remarkable level of detail was distinguishable, and variations in relative temperature were observed, so the proof-of-concept UAV flight indicates that this technology could be used to map spatial and temporal changes of the surface of tailings impoundment with a high level of detail.

Both the satellite and UAV data, when paired with atmospheric and ground data, show promise that they would enable the mapping of spatial and temporal changes in moisture content and strength across entire tailings impoundment(s).

\section{Procedure for Processing ASTER data to obtain Apparent Thermal Inertia} Purpose: Use when ASTER L1A data is available and map of spatial and temporal variations in surface temperature is the desired output.

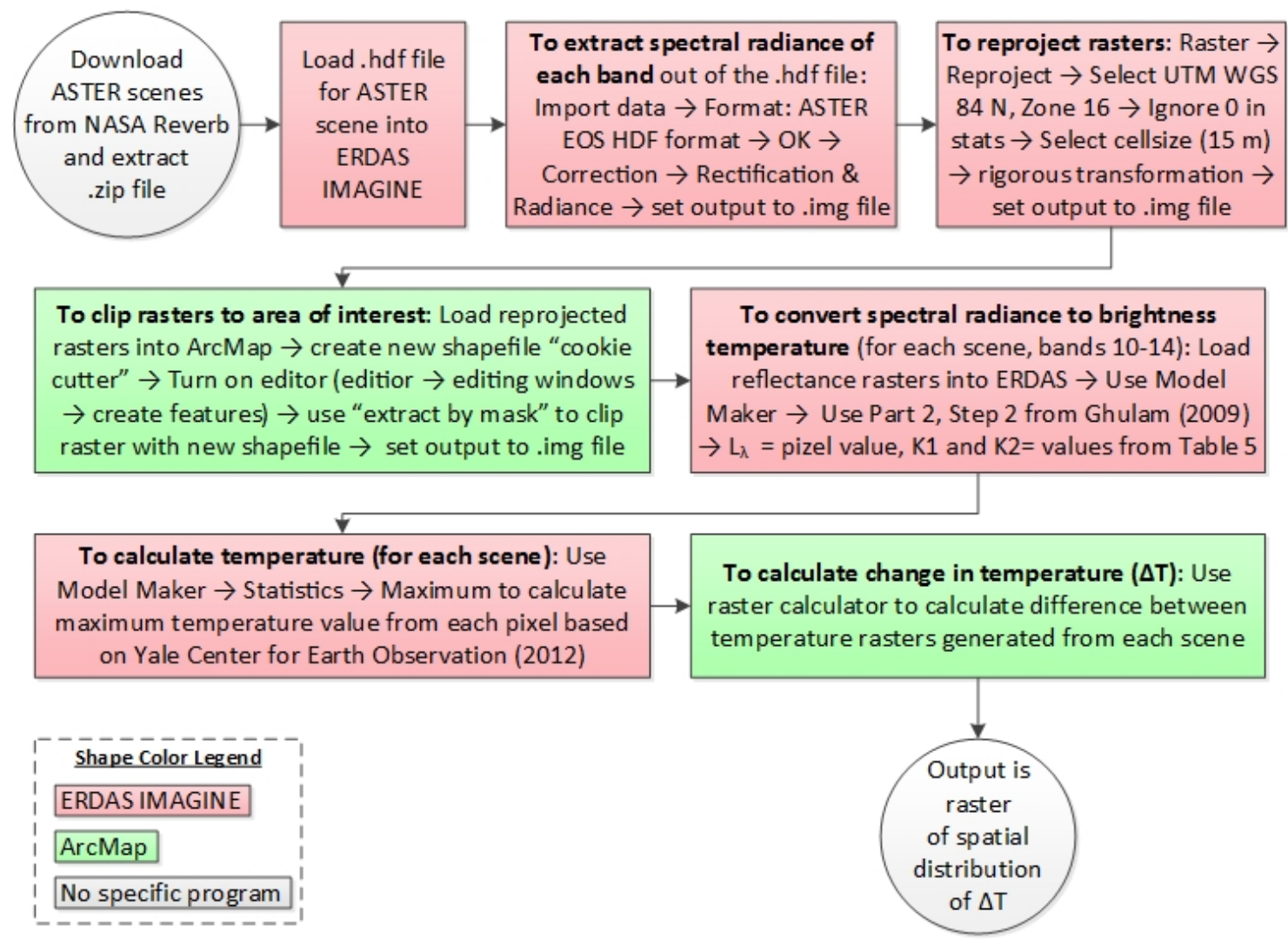

Figure 2-9 | Procedure used to process ASTER data to obtain $\Delta T$. 

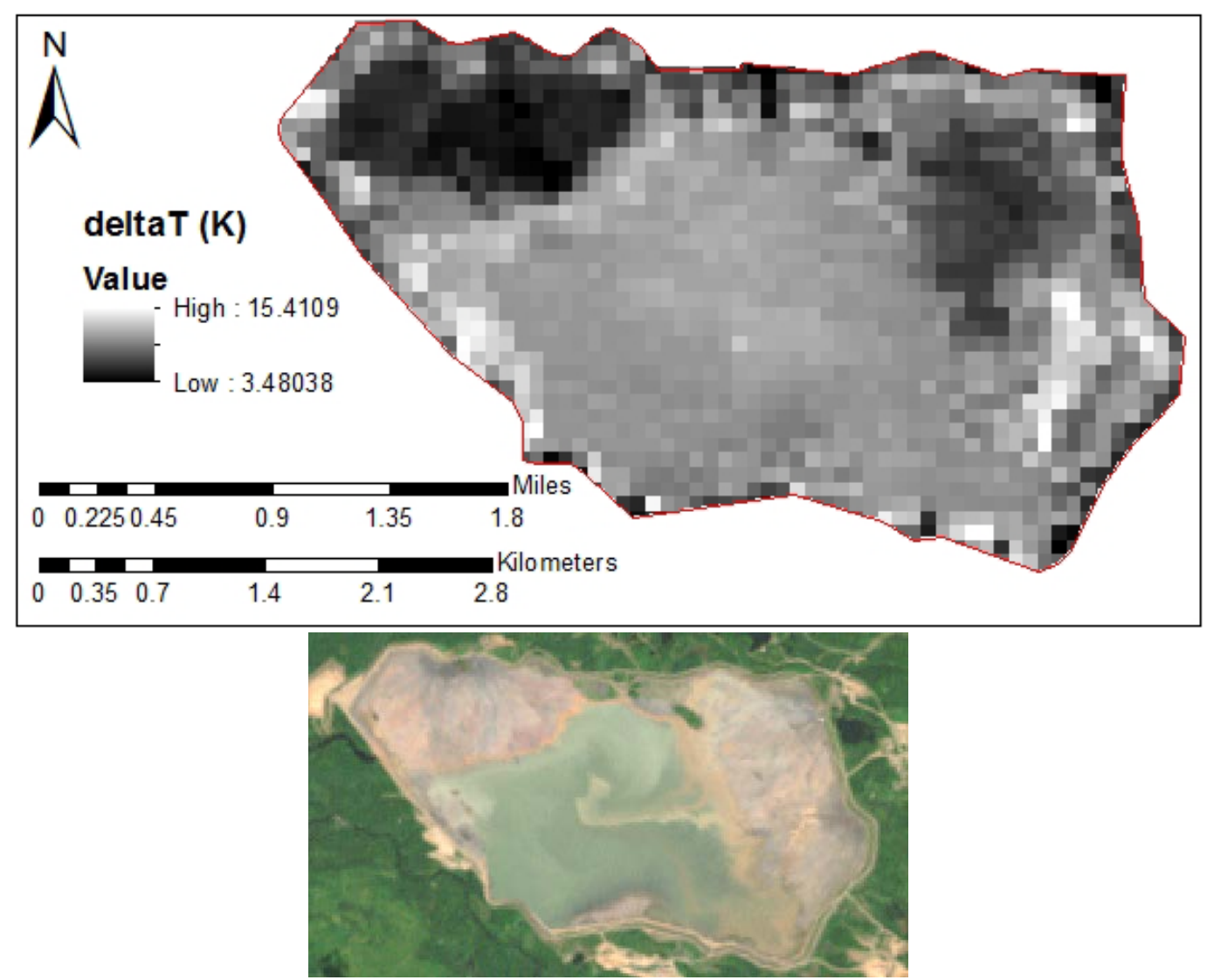

Figure 2-10 | Spatial variation in $\Delta T$ observed using ASTER data: (top) map of $\Delta T$ calculated using two ASTER scenes (April 23 and July 3, 2002), showing variation in $\Delta T$ can be detected; (bottom) real-color image showing impoundment at time of ASTER acquisition, showing water pond in center and unsaturated tailings on the east and west sides (data source: Landsat 7, image date July 28, 2002).
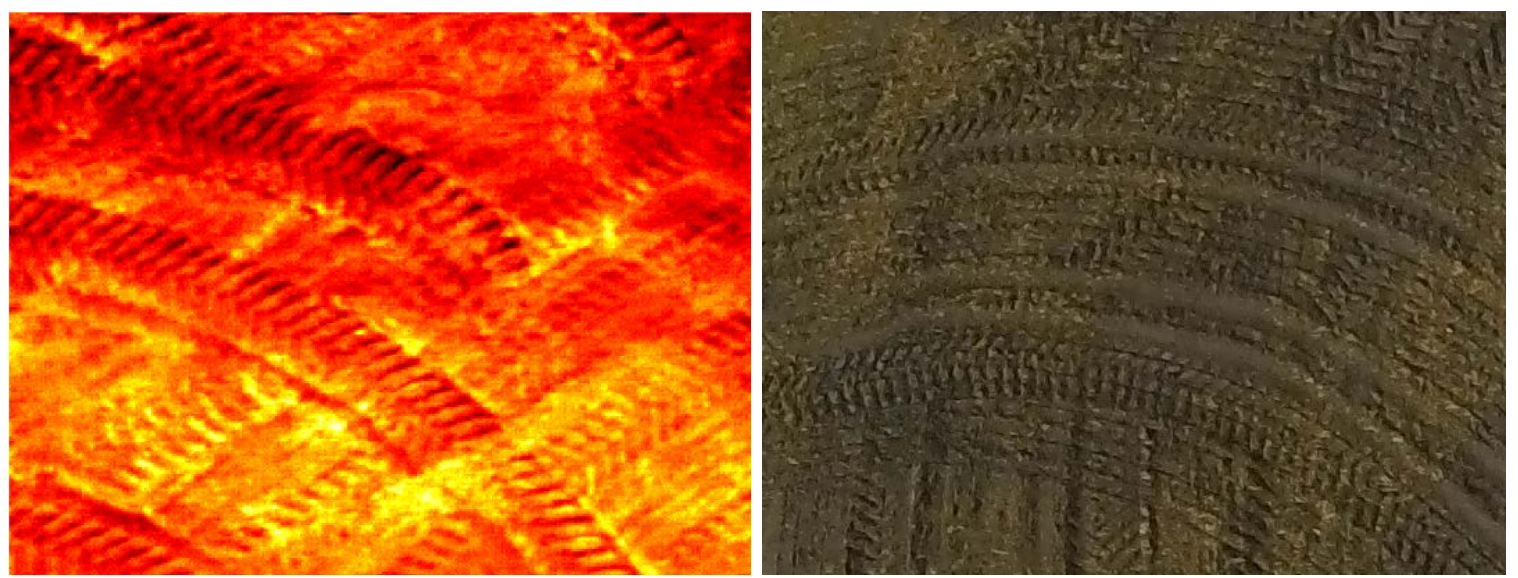

Figure 2-11 | Thermal infrared imagery (left) and visible imagery (right) of MN-mag tailings impoundment collected with UAV in May 2015. In the thermal imagery, dark colors represent lower relative temperatures, and bright colors represent higher relative temperatures. Detail including tire tracks can be distinguished in both images. 


\subsection{Methods to Satisfy Objective 3}

To satisfy Objective 3, laboratory methods to generate and quantify dust caused by sublimation for both $M I$-mag and $M N$-mag tailings were developed and implemented.

Because of a lack of existing research on generating ice lenses and dust via sublimation in laboratory settings, the final methods implemented in this study, described below, were developed through much trial-and-error of variables including freezing temperature and duration, presence/absence and temperature of circulating water bath below samples, and wind tunnel speed and duration. These methods were ultimately validated by comparing their appearance to frozen samples collected from the field and anecdotal descriptions of dust events at tailings impoundments.

\subsubsection{Sublimation of Laboratory Specimens}

When the behavior of cold-weather dusting due to sublimation was studied, laboratory specimens were treated in a way that was found to be representative of freezing and sublimation that is experienced at tailings impoundments.

All MI-mag and MN-mag samples were prepared as described in Section 2.1.2 to consistent density and moisture conditions. After samples were saturated with distilled water using Mariotte tubes, each sample was momentarily detached from its Mariotte tube and weighed so that moisture content and percent saturation could be calculated for the sample prior to freezing. If the sample height was not the same as the soil box (e.g. if the sample swelled due to saturation or if the sample settled due to soil suction), the sample height was measured with calipers and noted. Each Mariotte tube line was fed through a port in the side of the freezer, and reconnected to the base of the sample. Care was taken to reconnect samples to Mariotte tubes as quickly as possible, because samples settled and densified due to increased soil suction when disconnected for more than a few minutes. This process was repeated for each sample, and a total of up to six samples were loaded into the freezer at one time. 
Samples were then exposed to one-dimensional freezing from the surface down, which is representative of how the ground freezes, as peformed by Vitton and Muszynski (2013). To do this, a freezer, refrigerated recirculating chiller, and insulation were utilized. The freezer used was manufactured by Russells Technical Products (RB-3-1, serial 09982865 ), and featured temperature control from $-30^{\circ} \mathrm{C}$ to $80^{\circ} \mathrm{C} \pm 0.1^{\circ} \mathrm{C}$ and air circulation with dry air. There were two refrigerated recirculating chillers utilized for testing. The first was manufactured by PolyScience (5260P11A110B, serial 09982865), and features both a heating and cooling element with a working temperature range of $10^{\circ} \mathrm{C}$ to $40^{\circ} \mathrm{C} \pm 0.1^{\circ} \mathrm{C}$ and a flow rate of $3.8 \mathrm{LPM}$. The second unit was manufactured by Neslab Insturments (RTE-4 151120, serial 81F 7589-4), and also features both heating and cooling elements with a working temperature range of $-30^{\circ} \mathrm{C}$ to $100^{\circ} \mathrm{C} \pm 0.1^{\circ} \mathrm{C}$ and a flow rate of 13 LPM; this second unit was used when the PolyScience unit stopped working due to pump failure.

A metal water bath (dimensions $25 \mathrm{~cm} \mathrm{x} 43 \mathrm{~cm}$ x $20 \mathrm{~cm}$ ) surrounded with 2" Styrofoam insulation was connected to the refrigerated recirculating chiller with $1.2 \mathrm{~cm}$ inner diameter tubing. The water bath was placed into the freezer, and the lines connecting to the refrigerated recirculating chiller were wrapped in pipe insulation and run through the side port of the freezer. Samples were placed in the water bath onto wooden supports sufficiently tall to allow Mariotte tube fitting and lines to extend freely from the bottom of sample boxes. To ensure that a water source remained available for ice lens formation for the duration of sample freezing, a requirement for ice lens formation and dust generation via sublimation, the Mariotte tube bottles were kept outside of the freezer, and the lines were insulated with $1 / 4$ " thick pipe wrap insulation. Some of each line was also submerged in the antifreeze kept at $+5^{\circ} \mathrm{C}$ from the circulating water bath. The insulation was found to be sufficient to keep Mariotte tube lines from freezing. The water level in the Mariotte tube bottles was placed $5 \mathrm{~cm}$ above the tailings sample height to generate enough head to allow water to flow freely to the freezing front as ice lenses formed. Once all samples were placed into the water bath, the water bath was filled with $\mathrm{RV} \&$ Marine antifreeze $\left(-50^{\circ} \mathrm{F}\right.$ protection) to within $1-2 \mathrm{~cm}$ of the bottom 
of the sample boxes, without touching the soil boxes; this was to provide additional resistance to freezing for the Mariotte tube lines, and to keep the bottom of the tailings samples from freezing. Once the soil boxes were in place, the lines were insulated, and the antifreeze was in place, the void between the soil boxes and the edge of the water bath was filled with 2" Styrofoam insulation to prevent freezing of the samples from the sides. A schematic of the setup can be seen in Figure 2-12.

With the Mariotte tubes connected, insulated, and set to an appropriate height, the circulating water bath filled with antifreeze, and the samples insulated on all sides but the top, the samples were ready to undergo freezing/sublimation. The refrigerated recirculating chiller was set to $+5^{\circ} \mathrm{C}$, and the freezer was set to $-5^{\circ} \mathrm{C}$ with dry air and air circulation turned on. After 6 hours of freezing, which was found to provide enough time for the surface of all samples to completely freeze, the Mariotte tube bottles were raised an extra $2.5 \mathrm{~cm}$ to ensure that the water level remained above the sample surface. The samples were monitored periodically, and the water level in each Mariotte tube bottle was marked to monitor the volume of water being sucked into ice lens formation. The freezing/sublimation cycle lasted for 7 days. At the conclusion of the 7 day freezing/sublimation cycle, samples were ready for characterization of dust emissions.

After 7 days of freezing/sublimation, significant vertical expansion and dust generation occurred, so samples required extreme caution in handling while unloading from freezer and preparing for additional testing to avoid spilling of loose tailings. Due to the placement of Mariotte tube and water bath lines, it was easiest to remove samples in the back of the freezer first and move from back to front while unloading. It should also be noted that testing for one sample was completed before unloading a second sample from the freezer to avoid melting. Insulation surrounding the samples was carefully removed, and then one by one, samples were carefully detached from their Mariotte tube line and removed from the freezer. Upon removal, sample mass was measured using a balance, vertical expansion of the sample was measured with a caliper, ice lens structure and sample appearance was documented with digital photography, and then the sample was tested for erosion resistance using wind tunnel methods described in Section 2.4.2 
and/or ball drop methods described in Section 2.4.3. A summary of all measurements made before, during, and after freezing/sublimation for each sample is presented in Table $2-9$.

These laboratory methods to generate ice lens formation and sublimation for tailings samples were validated by visually comparing the appearance of the laboratory samples after treatment to frozen samples collected from the field. Photographs illustrating the ice structure in MI-mag laboratory samples are presented in Figure 2-13. For comparison, samples were collected from the MI-mag impoundment in January of 2016 using a pickaxe. Photographs were taken in the field, and the samples were transported back to the laboratory in a cooler to keep them frozen, where additional pictures were taken. The resulting images, shown in Figure 2-14, demonstrate the ice structure in the MI-mag field samples. Photographs were also taken in the field to document ice lens formation at the MN-mag impoundment in March of 2016, as shown in Figure 2-15. Comparing the laboratory and field images, it was concluded that the structure of the ice formed in the laboratory samples was comparable to the structure of the ice in the field samples. Accordingly, the laboratory methods for generating ice lenses and sublimation were deemed appropriate. 

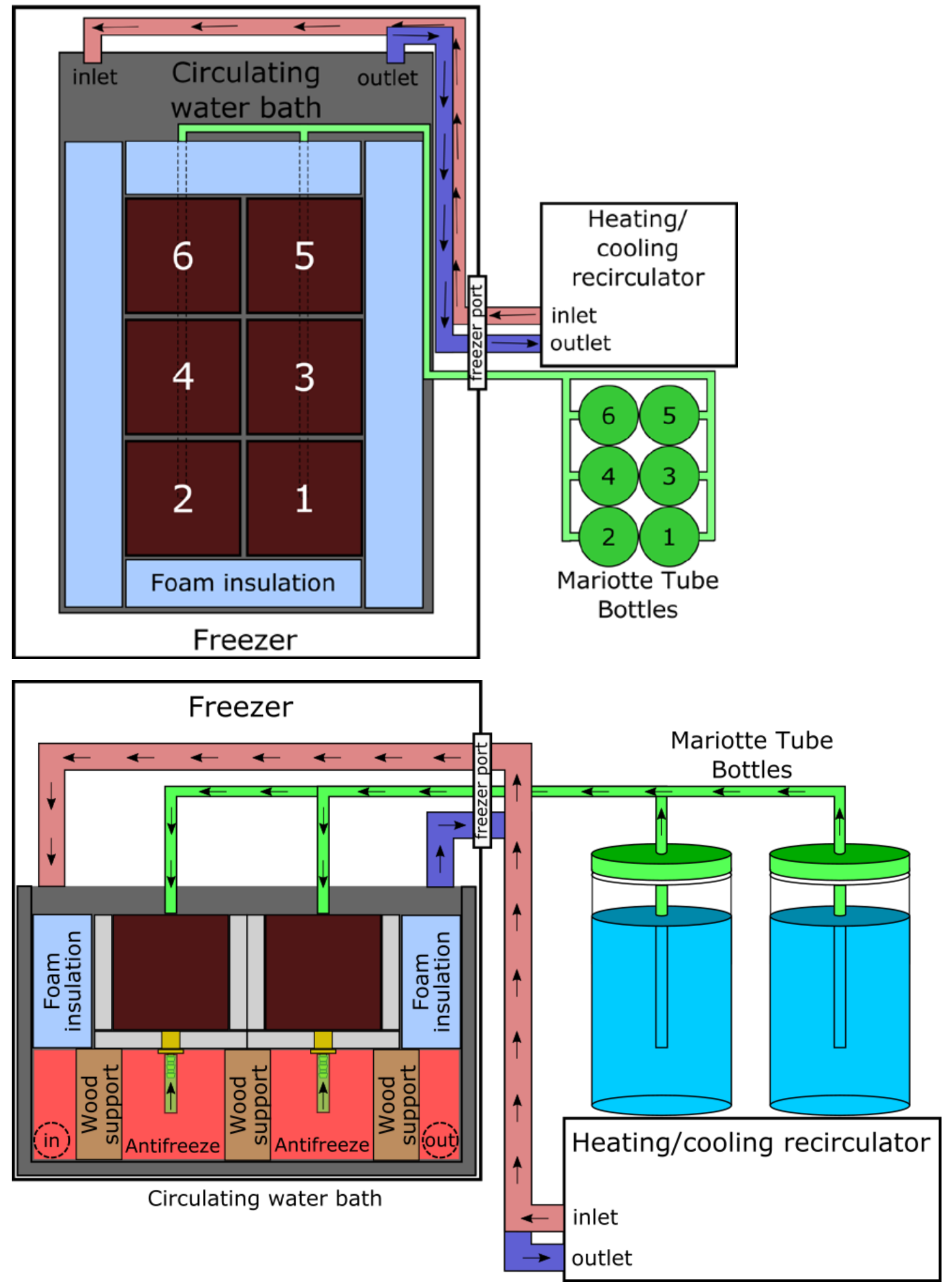

Figure 2-12 | Schematic of freezing/sublimation setup for MI-mag and MN-mag samples, with (top) plan view and (bottom) front view 
Table 2-9 | Description of measurements made during sample freezing/sublimation for MI-mag and MN-mag tailings.

\begin{tabular}{|l|l|l|l|}
\hline $\begin{array}{l}\text { Data } \\
\text { Collected }\end{array}$ & $\begin{array}{l}\text { Equipment } \\
\text { Used }\end{array}$ & Purpose & $\begin{array}{l}\text { No./Timing of } \\
\text { Measurements }\end{array}$ \\
\hline Sample mass & Balance & $\begin{array}{l}\text { To monitor sample mass (+ } \\
\text { changes due to ice lens } \\
\text { generation, - changes due to } \\
\text { ice sublimation) }\end{array}$ & $\begin{array}{l}\text { 1 measurement } \\
\text { before freezing, } \\
\text { measurement after } \\
\text { freezing }\end{array}$ \\
\hline $\begin{array}{l}\text { Sample } \\
\text { height }\end{array}$ & Digital caliper & $\begin{array}{l}\text { To quantify volume expansion } \\
\text { due to pore ice freezing and } \\
\text { ice lens formation }\end{array}$ & $\begin{array}{l}\text { 1 measurement } \\
\text { before freezing, } \\
\text { measurement after } \\
\text { freezing }\end{array}$ \\
\hline $\begin{array}{l}\text { Sample } \\
\text { temperature } \\
\text { profile }\end{array}$ & Thermocouples & $\begin{array}{l}\text { To quantify the depth and rate } \\
\text { of freezing (freezing front } \\
\text { propagation, ice lens } \\
\text { formation) }\end{array}$ & $\begin{array}{l}\text { Continuous } \\
\text { measurements } \\
\text { during freezing }\end{array}$ \\
\hline $\begin{array}{l}\text { Vol. of water } \\
\text { lost from } \\
\text { Mariotte } \\
\text { tube }\end{array}$ & $\begin{array}{l}\text { Bottle with } \\
\text { marked volume } \\
\text { gradations }\end{array}$ & $\begin{array}{l}\text { To quantify volume of water } \\
\text { used to feed ice lenses }\end{array}$ & $\begin{array}{l}\text { Measurements } \\
\text { before, periodically } \\
\text { during, and after } \\
\text { freezing }\end{array}$ \\
\hline $\begin{array}{l}\text { Digital } \\
\text { pictures }\end{array}$ & $\begin{array}{l}\text { Digital camera } \\
\text { formang document sample } \\
\text { appearance, change and ice } \\
\text { lens formation/structure }\end{array}$ & $\begin{array}{l}\text { Images before, } \\
\text { periodically during, } \\
\text { and after freezing }\end{array}$ \\
\hline
\end{tabular}



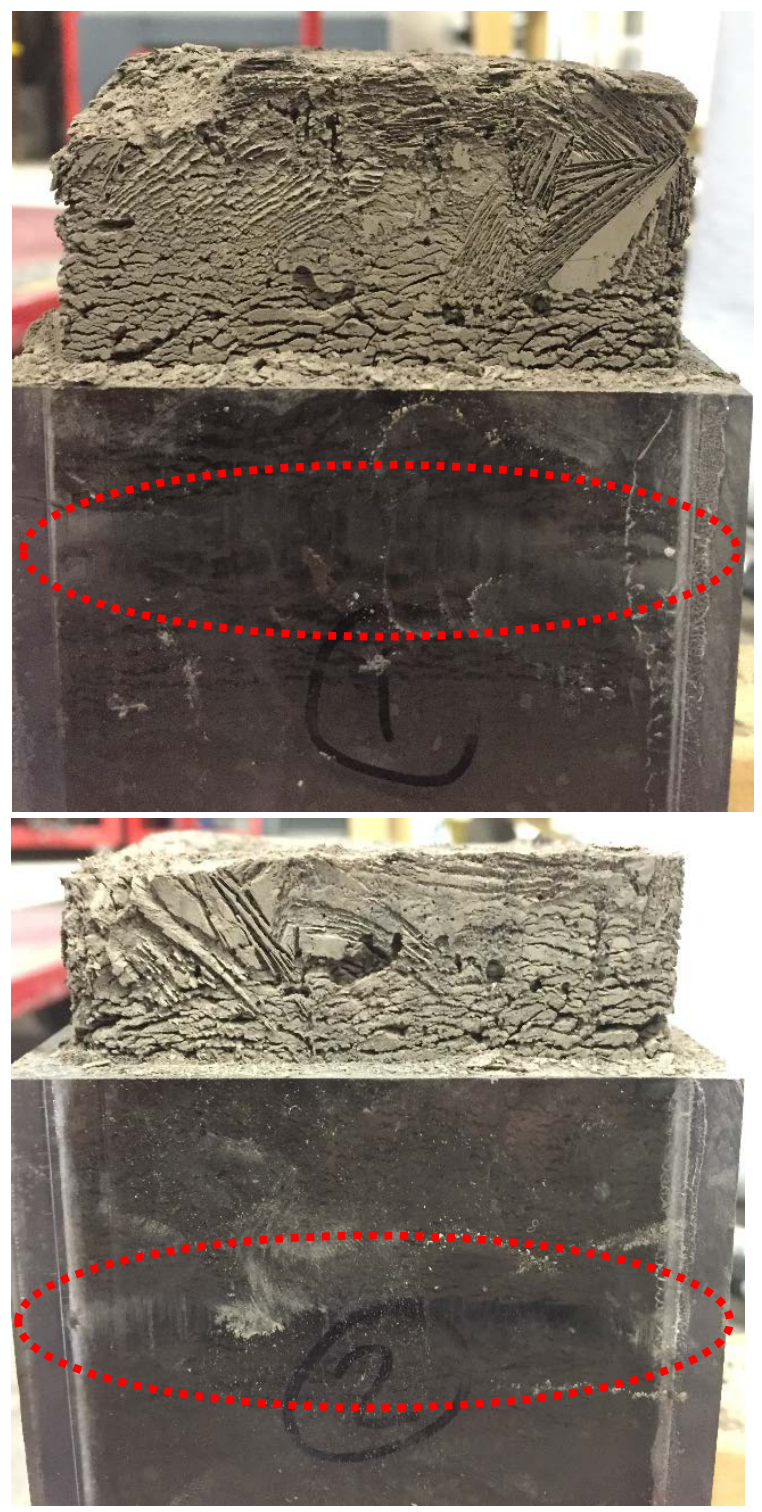

Figure 2-13 | Laboratory MI-mag tailings samples after freezing/sublimation, showing ice lens formation and structure. Small ice lenses are visible throughout samples, and large ice lenses are marked with red circles. 

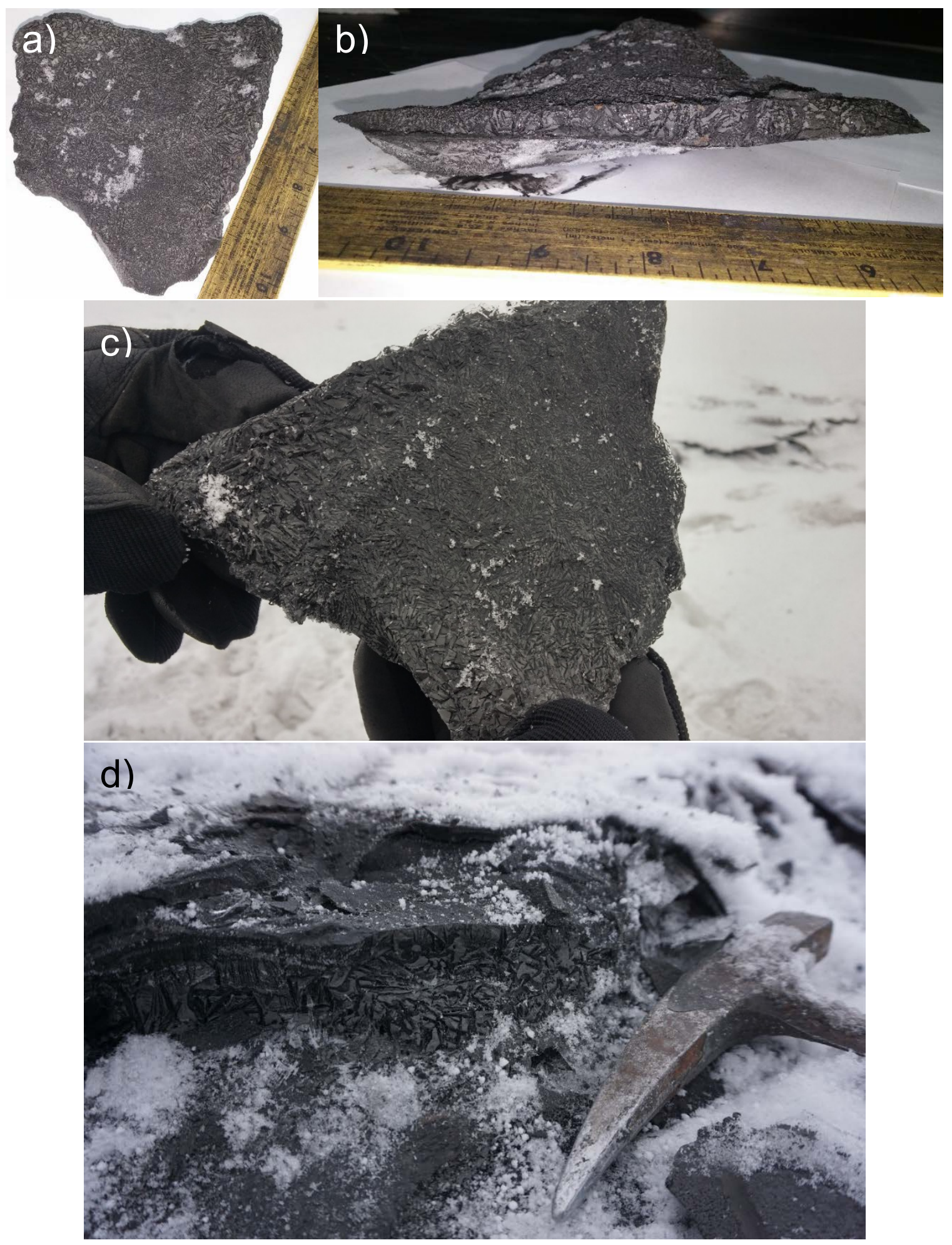

Figure 2-14 | Frozen field MI-mag tailings samples, showing ice lens formation and structure: (a) and (c) show ice lens structure on the tailings surface, (b) shows ice lens structure with depth as seen from the side, and (d) shows ice lenses structure in-situ. 


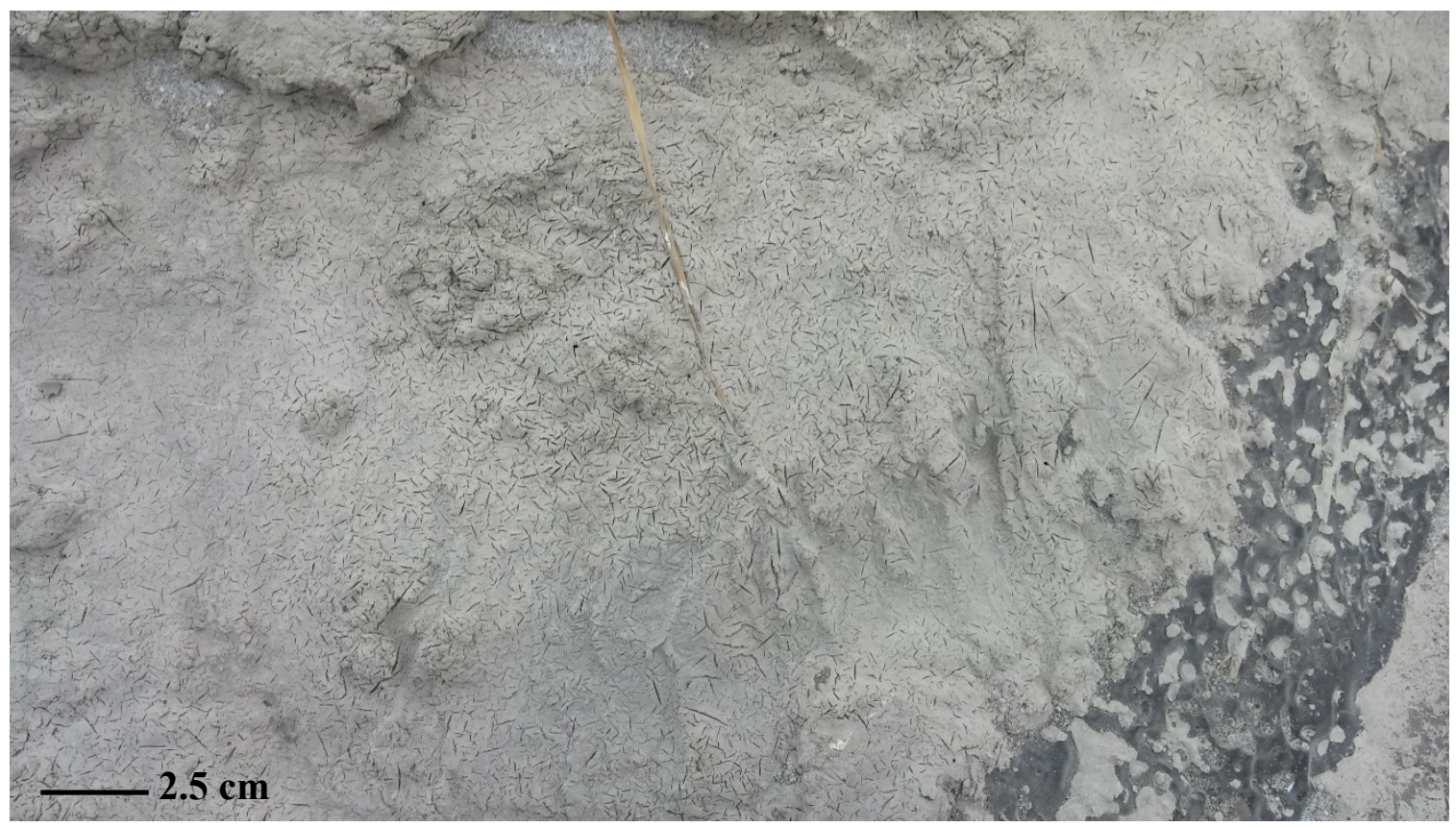

Figure 2-15 | Frozen field MN-mag tailings samples, showing ice lens formation and structure.

\subsubsection{Wind Tunnel Testing of Laboratory Specimens}

A wind tunnel, manufactured by Engineering Laboratory Design, Inc., was procured, serviced, and modified to fit the purposes of this research (Figure 2-16). The wind tunnel operates with suction and is capable of reaching wind speeds of $160 \mathrm{~km} / \mathrm{hr}(100 \mathrm{mph})$, which is well above the maximum operating speeds selected for this study of 60 to 100 $\mathrm{km} / \mathrm{hr}$ (or about 40 to $60 \mathrm{mph}$ ). The wind tunnel working section was fabricated using polycarbonate, with a $30.5 \mathrm{~cm} \times 30.5 \mathrm{~cm}$ (12 in x 12 in) cross section, and a length of 2.4 $\mathrm{m}(8 \mathrm{ft})$. A $15.25 \mathrm{~cm} \times 15.25 \mathrm{~cm}(6 " \times 6$ ") hole was cut in the base of the working section $1.8 \mathrm{~m}(6 \mathrm{ft})$ from the upwind end of the working section for loading test specimens. Plywood was affixed to the polycarbonate base of the working section, with the $1.8 \mathrm{~m}$ of working section leading up to the sample covered with 80 grit sandpaper (Klingspor's Woodworking 16" x 48" Aluminum oxide 80 grit wide belt, item WB39851, cut to size) to develop a turbulent boundary layer, based on the turbulent sections used by Han et al. (2009) and Okoli (2003). 80 grit sandpaper was selected for this purpose because it is 
similar in size to the particle sizes of tailings and would therefore simulate how wind would flow over a tailings impoundment.

Once the geometric modifications were made to the wind tunnel working section, the test conditions to be used were investigated. Test conditions considered were wind speed and test duration. Each of these conditions is discussed below. A saltating particle feed was not used in this study, because tailings particles are fine-grained and lightweight, and therefore stayed airborne in the wind tunnel rather than causing the desired abrasion to the surface that saltating particles provide. Rather than using a larger sand as a saltating particle feed, which would not be representative of the wind erosion mechanism acting on a tailings impoundment, the idea of using a saltating particle feed was abandoned.

The wind tunnel speed is controlled by motor frequency. To relate the motor frequency to wind speed at the sample location, a calibration curve was developed using an Extech Pitot Tube Anemometer + Differential Manometer. The calibration curve was generated for each of 8 evenly spaced locations laterally across the front of the $15.2 \mathrm{~cm}$ (6 in) sample opening in the bottom of the working section and for 3 locations vertically (top, middle, bottom). The maximum, minimum, and average wind speed in each location, in duplicate, was measured. Based on these measurements, a calibration curve was generated for predicting the wind speed samples were exposed to, using the average wind speed in the center of the front edge of the sample opening along the base (Figure 2-17). Maximum wind speeds at the tailings impoundments are expected to be between 60 and $100 \mathrm{~km} / \mathrm{hr}$ (or about 40 to $60 \mathrm{mph}$ ) (Price et al. 1997; Vitton 1997), so all wind tunnel testing was run at the upper end of this range, at $96.6 \mathrm{~km} / \mathrm{hr}(60 \mathrm{mph})$ or $41.4 \mathrm{~Hz}$.

Preliminary testing was conducted to assess the effects of timing when exposing frozen samples to these wind speeds. Based on these tests, too little exposure to wind resulted in no dust was loss, while too much exposure caused the sample to melt and run down the wind tunnel, thereby affecting mass loss measurements. Through trial and error testing, 30 seconds of testing at $41.4 \mathrm{~Hz}$ was found to generate dust without causing samples to melt. 
Prior to wind tunnel testing, each sample was first exposed to freezing/sublimation, as discussed in Section 2.4.1. After freezing/sublimation, significant vertical expansion and dust generation occurred, so samples required extreme caution in handling while unloading from freezer and preparing for additional testing to avoid spilling of loose tailings. Prior to loading, the sample mass was measured, and digital photographs were taken to document the condition of the sample, including any disturbance, prior to wind tunnel testing. Then, each sample was placed along the front side and in the center of the sample hole in the base of the wind tunnel supported by a lab jack. The sample was surrounded by a plywood plug to fill any voids between the sample box and the edge of the wind tunnel base, with the sample surface level and flush with the base of the wind tunnel. Then, the samples were exposed to 30 seconds of wind at $41.4 \mathrm{~Hz}$. After wind exposure, the sample was carefully removed from the wind tunnel so as to not cause sample disturbance and additional mass lost due to transport. After testing, sample mass was again measured to quantify mass lost in the wind tunnel, and additional pictures were taken to document any visible change to the sample that incurred during the wind tunnel testing. A summary of data collected for each sample tested in the wind tunnel is presented in Table 2-10.

While few direct accounts of the appearance of tailings during dust emissions are available, anecdotal sources report that a color change of the surface tailings typically precedes a dust event. Specifically, the color of the tailings gets lighter, likely due to sublimation of the pore ice and ice lenses, before dust is emitted. Interestingly, although the methods described in this section do not often yield massive quantities of dust, they do cause a similar color change on the tailings surface (i.e., the color gets lighter), which is indicative that the conditions in the laboratory are similar to those experienced at active tailings impoundments. 


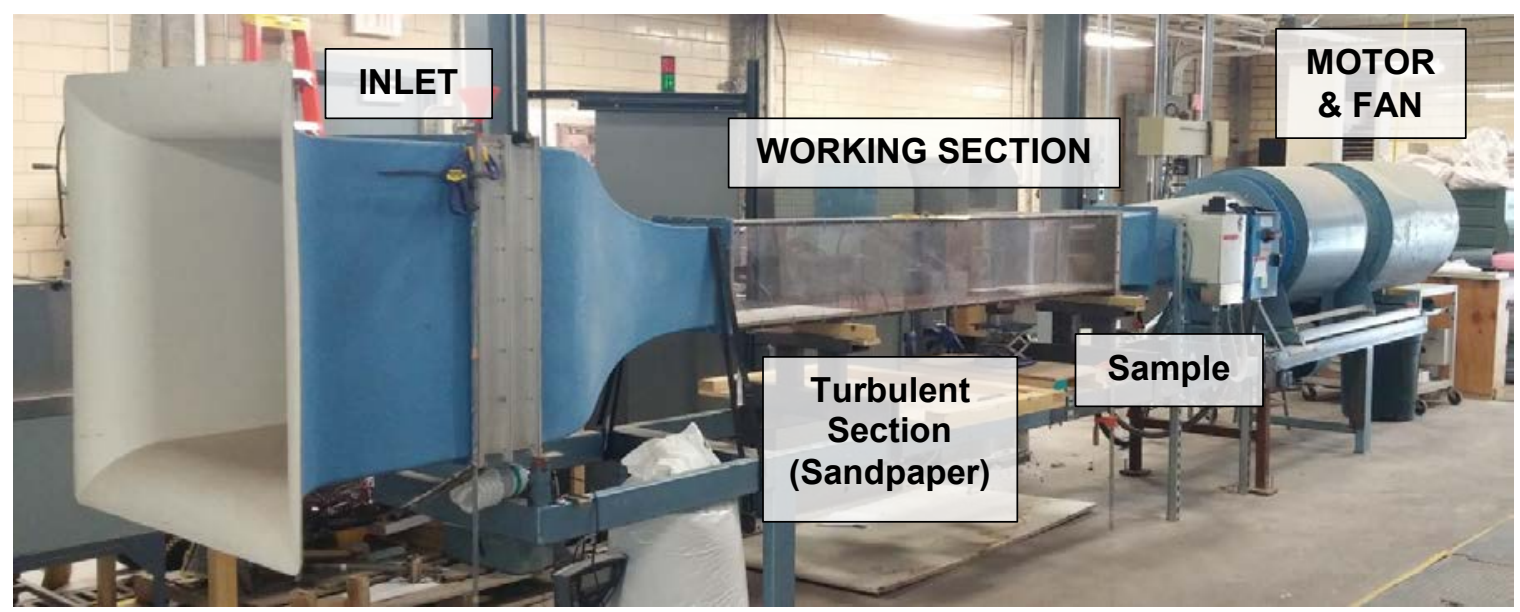

Figure 2-16 | Wind tunnel used at Michigan Technological University, Houghton, MI.

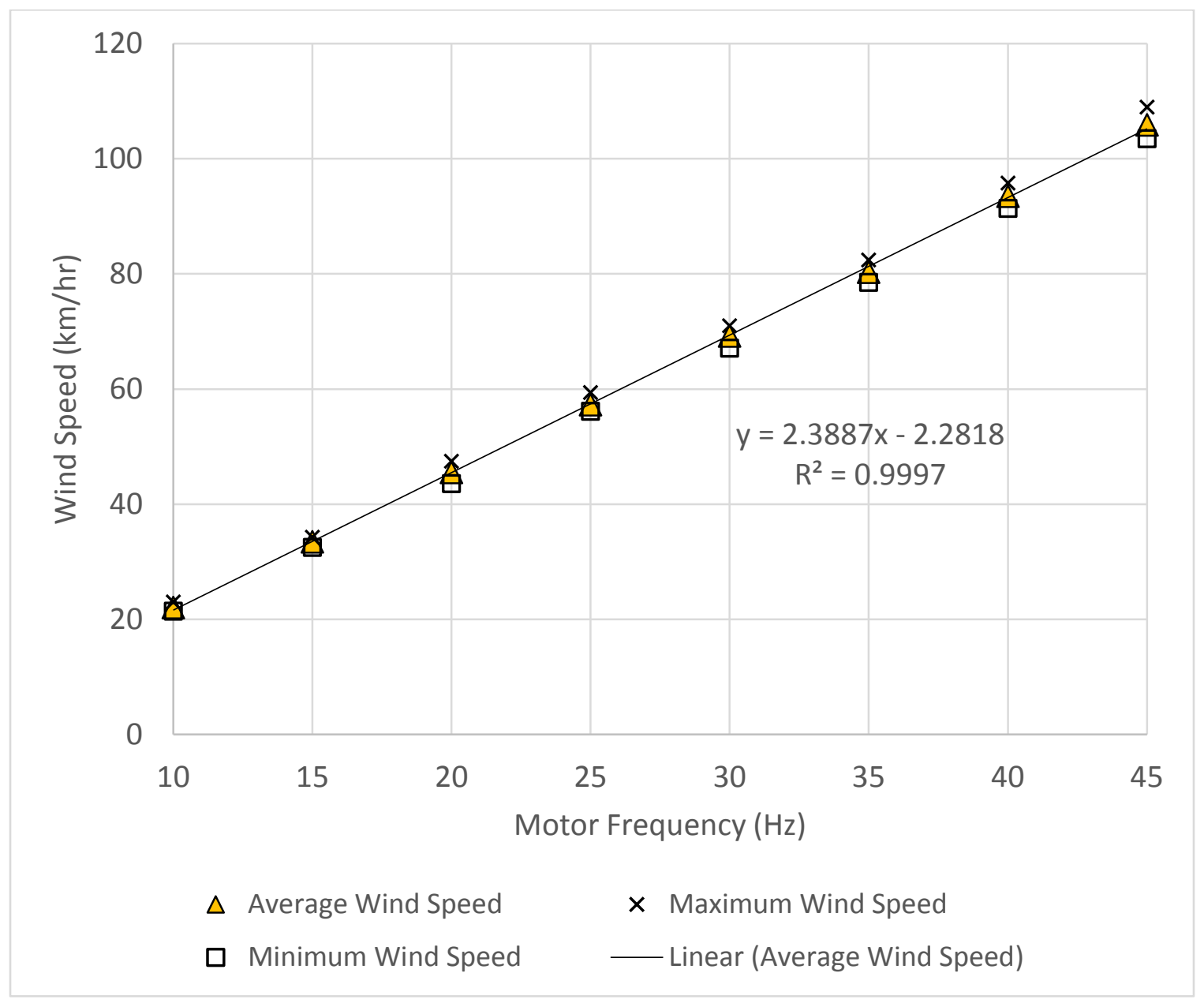

Figure 2-17 | Wind speed calibration curve for wind tunnel based on average wind speed over 30 seconds at sample location with sandpaper base. 
Table 2-10 | Description of measurements made during wind tunnel testing for MImag and $\mathrm{MN}$-mag tailings.

\begin{tabular}{|l|l|l|l|}
\hline $\begin{array}{l}\text { Data } \\
\text { Collected }\end{array}$ & $\begin{array}{l}\text { Equipment } \\
\text { Used }\end{array}$ & Purpose & $\begin{array}{l}\text { Timing of } \\
\text { Measurements }\end{array}$ \\
\hline Sample mass & Balance & $\begin{array}{l}\text { To monitor sample mass lost } \\
\text { due to wind erosion }\end{array}$ & $\begin{array}{l}\text { Before + after wind } \\
\text { tunnel testing }\end{array}$ \\
\hline $\begin{array}{l}\text { Digital } \\
\text { pictures }\end{array}$ & $\begin{array}{l}\text { Digital } \\
\text { camera }\end{array}$ & $\begin{array}{l}\text { To document change in sample } \\
\text { color and appearance }\end{array}$ & $\begin{array}{l}\text { Before + after wind } \\
\text { tunnel testing }\end{array}$ \\
\hline Video & Video camera & $\begin{array}{l}\text { To document effects of wind on } \\
\text { sample appearance/structure }\end{array}$ & $\begin{array}{l}\text { During wind tunnel } \\
\text { tesitng }\end{array}$ \\
\hline
\end{tabular}

\subsubsection{Ball drop testing}

To provide continuity with an associated research project, and to provide an alternative metric to assess the strength performance of tailings exposed to freezing/sublimation, a novel test developed by Buikema (2015) and Buikema et al. (accepted), called the steel bearing drop test or ball drop test, was implemented. The method is discussed at depth in Buikema (2015), but is outlined below.

For samples that did not have visibly notable surface disturbance due to wind tunnel testing (described in Section 2.4.2), ball drop testing followed wind tunnel testing. Ball drop testing was also conducted on several samples without being previously tested with the wind tunnel, and results were comparable. For each sample tested, a $3.4 \mathrm{~g}$ steel ball bearing with a diameter of $0.945 \mathrm{~cm}$ was dropped onto the soil surface from a height of $1.5 \mathrm{~m}$. The ball bearing was removed with a magnet, and the impact crater left behind on the soil surface by the ball bearing was measured in the $\mathrm{x}$ and $\mathrm{y}$ axis using a digital caliper; these two crater diameter values were averaged together to report a single average crater diameter. This was repeated 5 times for each sample, with one impact crater in each quadrant of the sample surface, and one impact crater in the center of the sample surface. 


\subsection{Methods to Satisfy Objective 4}

To satisfy Objective 4, biological soil crusts were grown on MI-mag/MN-mag blend tailings. Subsequently, the biomass of the crusts was quantified, the wind erosion resistance was tested through laboratory wind tunnel experiments, and the strength of the biological soil crust was quantified via the ball drop test.

\subsubsection{Proof-of-Concept Growth of Biological Soil Crusts on tailings}

Prior to doing extensive tests on the effects of biological treatments on tailings, proofof-concept testing was conducted to verify that biological soil crusts can be grown on iron mine tailings.

For this demonstration, putative cyanobacterial cultures were used for inoculum; these culutures had been isolated from native biological soil crusts from the Upper Peninsula of Michigan. Biological soil crust samples were collected on November 4, 2012 from the Black Creek Nature Sanctuary and on June 28, 2014 from the Eagle River beach. Crust samples were aseptically transferred to petri dishes containing MI-mag and MI-hem tailings saturated with BG-11 growth medium (Stanier et al. 1971) by breaking up the crust by hand or with a sterile mortar and pestle, and sprinkling the crust over the tailings surface. Approximately 1 gram of crust was transferred to every plate. The petri dish samples were subsequently moistened with water once per week, and given a dose of BG-11 medium once per week. The samples were kept in a Conviron BDR16 growth chamber $\left(20^{\circ} \mathrm{C}\right.$ with full spectrum lights) until September 2013 , after which they were moved to a window for a source of natural light and kept at room temperature. Growth was monitored visually. Notable crust growth and development occurred on MI-hem and MI-mag tailings after four to six months of maintenance, as shown in Figure 2-18. This successfully proved that biological soil crusts can grow on iron mine tailings. 

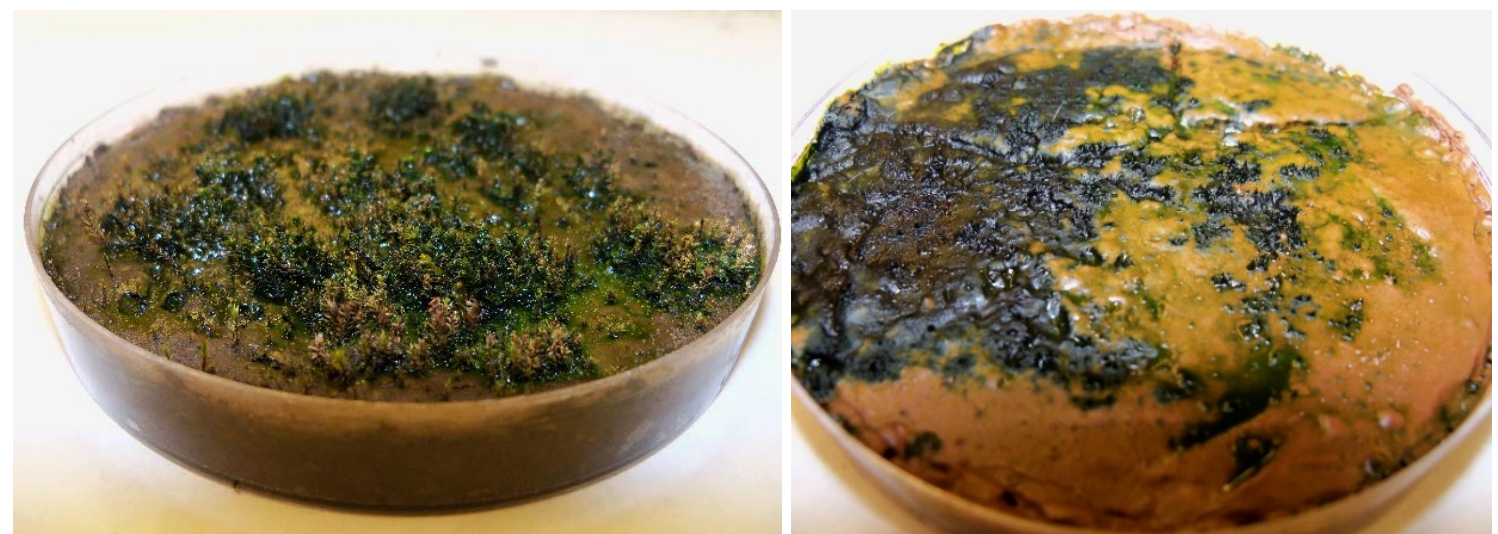

Figure 2-18 | Biological soil crusts growing on MI-mag (left) and MI-hem (right) tailings surface.

\subsubsection{Biological Soil Crusts organism selection}

Once it was demonstrated that biological soil crusts could successfully grow on iron mine tailings, additional analysis was conducted to select one or more cultures suitable for use as a pioneer organism in further experimentation.

As discussed in Section 1.3.4, there are many benefits associated with having a biological soil crust with filamentous cyanobacteria as the pioneer organism. Therefore, the native biological soil crusts that grew successfully on the mine tailings and that appeared to be cyanobacteria based on a visual assessment (e.g., black color and flat appearance) and a preliminary microscopic evaluation were selected for further study. Specifically, three putative cyanobacteria crust samples were studied further by: (1) transferring the crust cultures to liquid culture flasks containing aqueous BG-11 growth medium; (2) transferring the cultures to new petri dishes containing MI-hem or MI-mag tailings, as well as to BG-11 agar plates; and (3) observing the cultures under a light microscope. Each of these techniques is described in more detail below.

First, the growth of three selected crust samples in aqueous solution was attempted. The goal was to grow a dense liquid cultures that could be used as an inoculum and decrease the time required to develop a crust on the tailings from 4-6 months down to the order of weeks. To do this, a protocol for growing cyanobacteria in aqueous solution was 
developed based on the work of Gerhardt et al. (1994); Liu et al. (2008); McKenna Neuman and Maxwell (1999); McKenna Neuman and Maxwell (2002); McKenna Neuman et al. (2005); McKenna Neuman et al. (1996); O'Brien and McKenna Neuman (2012); and Waterbury (2006). Erlenmeyer flasks (500 mL) were first aseptically filled to $20 \%$ of their total volume with stock BG-11 growth medium, and the flask mouth was closed with a sterile cotton plug. Crust samples of approximately 1 gram were then taken from the petri dish samples and placed into the flasks. The flasks were subsequently incubated in a shaking water bath at $30^{\circ} \mathrm{C}$ under full-spectrum lights (14 hours of light/10 hours of dark), and the growth was monitored visually. Unfortunately, the high concentration of tailings in the initial flasks made it difficult to distinguish cyanobacterial growth. Therefore, $10 \mathrm{~mL}$ of each culture was aseptically transferred to new a flask after approximately 2 weeks of growth. The new flasks were incubated under the same conditions, and all three putative cyanobacteria samples thrived in this environment based on visual assessment.

Secondly, after approximately 3 weeks of growth, the second liquid culture flasks were used to inoculate new petri dishes containing MI-mag and MI-hem tailings. In addition, the liquid cultures were used to try to isolate pure cultures on BG-11 agar plates.

For the tailings plates, $10 \mathrm{~mL}$ of the liquid culture was transferred via pipette to the tailings. Growth on tailings plates took 5-6 weeks to appear, and 8-9 weeks to develop (as shown in Figure 2-19). BG-11 agar plates were prepared based on Gerhardt et al. (1994), combining BG-11 medium with 2\% agar as follows: $500 \mathrm{~mL}$ of BG- 11 medium was autoclaved, and separately $500 \mathrm{~mL}$ of distilled water was mixed with $20 \mathrm{~g}$ agar and then autoclaved; when the solutions cooled to $50^{\circ} \mathrm{C}$, they were combined and then plates were poured. To inoculate the BG-11 agar plates, $5 \mathrm{~mL}$ of the flask culture was transferred via pipette onto the surface of the agar plates. The inoculated petri dishes with tailings and agar substrates were kept in clear plastic tubs at room temperature under full-spectrum lights (14 hours of light/10 hours of dark), with growth monitored visually. The agar plates demonstrated rapid growth, with most plates being completely covered in 5 weeks. 
Subsequently, biomass samples from the existing agar plates were used to inoculate new agar plates by transferring the biomass with micro-forceps. However, it became apparent that transferring biomass from agar plate to agar plate was much less effective for cyanobacterial growth than transferring from liquid to plate. For this reason, the agar plates were abandoned and a new set of liquid culture flasks was prepared using the original flasks as an inoculum. In an attempt to isolate the cyanobacteria from the mixed cultures based on the methods suggested by Waterbury (2006), filaments were first transferred from the original flask to a small beaker filled with DI water using a pipette. The beaker was agitated with stirring/shaking to attempt to wash off any unicellular organisms before the filaments were transferred to the new flask with tweezers. While cyanobacteria isolation by this method was not successful, the organisms that make up the three native cultures grow well on the iron tailings, and maintaining the samples in liquid medium has proven to be a successful way to grow and maintain these native organisms.

Lastly, the three native biological soil crust samples from the original flasks and original tailings plate were observed under a light microscope. Wet mounts were prepared for each sample, each sample was viewed under 10x and 40x magnification, and photographs were taken (see Figure 2-19). Based on the microscopic analysis, there are filaments present in each sample, but there are also other organisms still present. The filaments, presumably filamentous cyanobacteria, dominate the samples, and therefore further isolation was not attempted.

All three native crust samples that were evaluated demonstrated growth in liquid medium and on iron tailings samples, and appeared to be primarily filamentous cyanobacteria when studied under a light microscope. However, based on the rapid rate of growth and almost complete coverage of the tailings plate, and the interaction between filaments observed under the microscope (Figure 2-19), one of the three native samples, the Eagle River Crust, was selected as the native organism that would be used for further study. 

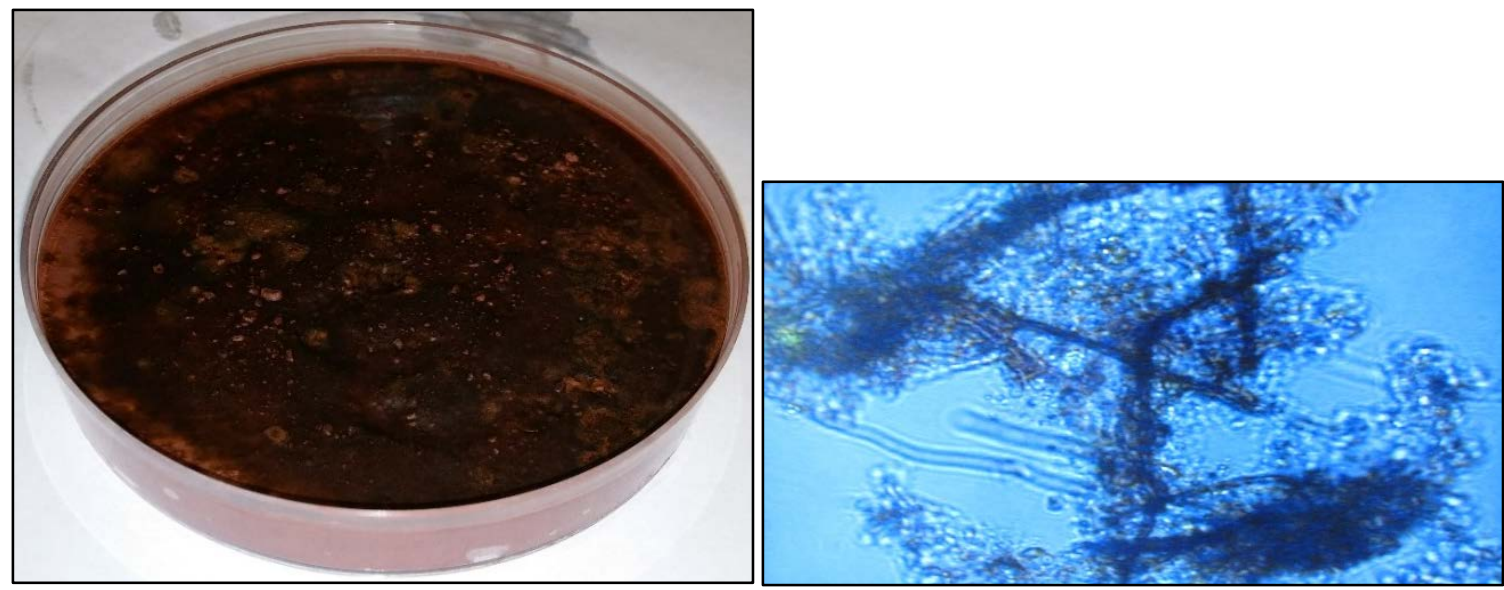

Figure 2-19 | Eagle River crust growing on MI-hem tailings (left) and viewed under light microscope at 40x magnification (right). It should be noted that the MI-hem tailings are naturally red, and the crust is black, showing almost total coverage of tailings.

The Eagle River crust culture has two attributes that make beneficial for use in this study. First, because it was isolated from the local area, it can survive the harsh winters that the MI-mag, MI-hem, and MN-mag impoundments can experience. Secondly, it has proven to grow quite successfully on tailings from these impoundments. However, the organism(s) that comprise the culture are unknown, and filamentous cyanobacteria are not the only organisms present.

To serve as a point of comparison for the performance of the native Eagle River crust, a pure culture of Anabaena doliolum, a filamentous cyanobacterium that has been shown to produce EPS (De Philippis et al. 2001), was purchased from the American Type Culture Collection (ATCC 43530). The freeze-dried culture was re-constituted using the recommended BG-11 medium and ATCC protocol. Specifically, the freeze-dried tube was thawed at room temperature, and then the entire contents were aseptically transferred via pipette to a test tube containing $5 \mathrm{~mL}$ of BG-11 medium. This test tube was mixed for 15 seconds using a vortex mixer. Then $0.5 \mathrm{~mL}$ was transferred from the first tube to each of three test tubes containing $5 \mathrm{~mL}$ of BG-11 medium. The test tubes were placed on an angle in a test tube rack under full-spectrum lights at approximately 2000 lux on a 14 hour light/10 hour dark cycle. Every 2-3 weeks, $0.5 \mathrm{~mL}$ from each tube was transferred to a fresh tube with $5 \mathrm{~mL}$ of BG-11 medium to ensure fresh growth medium for healthy cells. For long term storage, $0.5 \mathrm{~mL}$ from the freshest tubes was transferred to BG-11 
agar slants, which were prepared using the same recipe as the BG-11 agar plates (described above), and once the tubes were poured, they were tilted so the agar cooled on a slant. The inoculated agar slants were also stored under full spectrum lights at approximately 2000 lux on a 14 hour light/10 hour dark cycle. The liquid tubes served as inoculum for liquid culture flasks that were then used to inoculate bioreactors.

No proof-of-concept testing was performed on the $A$. doliolum. The same test methods developed for the Eagle River crust (discussed in the following sections) were also applied to this pure culture. However, while this organism, isolated from a rice field in India, grew well in liquid BG-11 and on BG-11 agar slants, it did not grow well on tailings. This demonstrated that not all biological soil crust organisms are suitable for crust development on tailings.

\subsubsection{Bioreactor Inoculation and Maintenance}

As described in Section 2.4.2, growing and maintaining the biological soil crust cultures in liquid BG-11 medium proved to be effective for both the Eagle River crust culture and the $A$. doliolum culture. Thus, liquid cultures were used to inoculate the bioreactors. Four sets of six bioreactors were inoculated: (1) a set inoculated with $A$. doliolum, (2) a set inoculated with the Eagle River culture and maintained with similar timing to the A. doliolum bioreactors, (3) a second set inoculated with the Eagle River culture that was allowed to grow for a longer time, and (4) a control set with no inoculum.

The general procedure for bioreactor inoculation was as follows: (1) the inoculum was grown, (2) the soil boxes were packed with tailings, (3) the bioreactors were inoculated with the sample, and (4) the bioreactors were maintained and monitored. Specific details about each step follow.

To grow the inoculum, Erlenmeyer flasks filled with sterile BG-11 to $20 \%$ of their total volume were inoculated with at least $2.5 \mathrm{~mL}$ of the Eagle River sample from a previous flask, and the flask was covered with a sterile cotton plug. The flasks were subsequently incubated in a shaking water bath at room temperature under full-spectrum 
lights (14 hours of light/10 hours of dark) at a brightness of approximately 1000 lux. The cultures were monitored visually, and digital photos of the flasks were taken weekly to track culture growth in the flasks.

The soil boxes used as bioreactors were prepared following the procedures described by Buikema et al. (accepted) and Zwissler et al. (in preparation), as summarized here. All bioreactors were packed into $75 \mathrm{~mm}$ x $75 \mathrm{~mm}$ x $75 \mathrm{~mm}$ soil boxes with MI-mag/MN-mag blend tailings to a void ratio of 0.9 , and saturated with autoclaved, distilled water. After saturation and immediately preceding inoculation, the Mariotte Tubes were disconnected from the bioreactors so that they could drain freely and the inoculum could percolate through the tailings. Also before inoculation, a $1 \mathrm{~cm}$ high polycarbonate collar was attached to the top of each soil box using Gorilla Tape and silicone caulk to provide extra headspace for containing the inoculum until it could permeate through the tailings. After treatment, the collar was removed by cutting through the tape with a razor blade, leaving the soil surface flush with the top of the soil box.

Once the inoculum was grown and the soil boxes were prepared and saturated, the bioreactors were inoculated, as shown in the schematic in Figure 2-20. For each set of Eagle River inoculum, the contents of the inoculum flask(s) were poured into an autoclaved blender, and sterile BG-11 medium was added (if needed, as described in Table 2-11) to either dilute the inoculum to increase the volume applied to each soil box to ensure total bioreactor coverage, or to visibly match the biomass density of the previous bioreactors, as verified by comparing samples to photographs of previous inoculum and measuring the absorbance at three critical wavelengths (660, 700, and 860 $\mathrm{nm}$ ) using a spectrophotometer; these wavelengths were selected due to their relevance to chlorophyll and biomass monitoring (Thenkabail et al. 2011). The contents were covered and blended at a medium to high speed setting for 2 minutes, which was selected to allow for thorough mixing while minimizing damage to the organisms (Lindahl and Bakken 1995). A $10 \mathrm{~mL}$ pipette was used to transfer $20 \mathrm{~mL}$ of inoculum from center of liquid column to each soil box. Care was used when pipetting the inoculum onto the soil surface as to not cause unnecessary surface erosion from the stream of liquid hitting the tailings. After $20 \mathrm{~mL}$ of inoculum was distributed to each bioreactor, the blender was run on 
medium to high speed for another 10 seconds to re-suspend the particles, and the process of distributing inoculum via pipette and re-suspending particles in the blender was repeated until all inoculum was used or the desired volume of inoculum was distributed to each soil box. For the control set of bioreactors, no blending was required, and a total of $33 \mathrm{~mL}$ of sterile BG-11 was added to the top of the bioreactors via pipette and allowed to permeate through the sample in the same was the inoculum did for the treated samples. While the inoculum or growth medium was percolating through the bioreactors, they were covered with a square piece of sterile gauze to minimize contamination. Once the inoculum or growth medium was absorbed completely, each set of bioreactors was transferred to a clear, plastic tub with the lids loosely placed on top to allow for air circulation while keeping possible contamination to a minimum.

Inoculated and control bioreactors were then maintained and monitored. All bioreactors were stored in plastic tubs with the lids loosely placed on top to allow for air circulation while keeping possible airborne microbial contamination to a minimum. The bioreactors were incubated at room temperature under 1600 to 1800 lux of full spectrum light on a 14 hour light/10 hour dark cycle, as recommended for A. doliolum by ATCC. The bioreactors were monitored and maintained two times per week. On each occasion, the samples were first monitored by taking digital photographs of the sample surface to qualitatively monitor crust growth and development. In addition, for quantitative monitoring, an ASD FieldSpec ${ }^{\circledR}$ HandHeld Pro Spectroradiometer (325-1075nm) with plant probe was used to collect multispectral reflectance data for the crusts. For each sample, the average of 10 spectral reflectance curves collected at two different locations on the sample surface ( 5 curves per location) was used to monitor biomass with vegetation indices, including the Normalized Difference Vegetation Index, or NDVI (Thenkabail et al. 2011). After completing the monitoring, the samples were maintained by applying BG-11 medium once per week, and sterile distilled water once per week. In each case, the fluid was applied using a squirt bottle, taking care not to cause surface erosion. Sufficient fluid was applied to keep the samples moist, but not saturated. 
Bioreactors were maintained and monitored until biological soil crusts were sufficiently developed, at which time the biomass was quantified (Section 2.4.4) and the biological soil crust resistance to freezing/sublimation and wind erosion was tested (Section 2.4.5). For each set of six bioreactors, one was used for biomass characterization, and the remaining five were used to study wind erosion resistance.

Table 2-11 | Summary of inoculum for ER1, ER3, and ER4 bioreactors, including absorbance used to compare inoculum biomass density

\begin{tabular}{|c|c|c|c|c|c|c|c|}
\hline Inoculum & $\begin{array}{l}\text { Vol. } \\
\text { from } \\
\text { flask(s) } \\
(\mathrm{mL})\end{array}$ & $\begin{array}{l}\text { Vol. } \\
\text { after } \\
\text { dilution } \\
(\mathrm{mL})\end{array}$ & $\begin{array}{l}\text { Reason for } \\
\text { inoculum } \\
\text { dilution }\end{array}$ & $\begin{array}{l}\text { Vol. of } \\
\text { inoculum } \\
\text { per box } \\
(\mathrm{mL})\end{array}$ & $\mathrm{A}_{660}$ & $\mathrm{~A}_{700}$ & $\mathrm{~A}_{860}$ \\
\hline ER1* & 400 & 400 & $\mathrm{n} / \mathrm{a}$ & 66 & - & - & - \\
\hline ER3 & 100 & 200 & $\begin{array}{l}\text { Matching } \\
\text { biomass density } \\
\text { of ER1 }\end{array}$ & 33 & 1.361 & 1.158 & 0.821 \\
\hline ER4 & 100 & 240 & $\begin{array}{l}\text { Increase volume } \\
\text { of inoculum }\end{array}$ & 40 & 0.749 & 0.630 & 0.423 \\
\hline $\begin{array}{l}\text { ER4- } \\
\text { reinoc** }\end{array}$ & 100 & 300 & $\begin{array}{l}\text { Increase volume } \\
\text { of inoculum }\end{array}$ & 50 & 0.624 & 0.584 & 0.395 \\
\hline \multicolumn{8}{|c|}{$\begin{array}{l}\text { * The biomass of ER1 was not quantitatively measured, but was rather documented in } \\
\text { photographs showing the color of the inoculum. ER } 3 \text { inoculum was diluted to visually } \\
\text { match the color of the ER1 inoculum, and then biomass was roughly characterized using } \\
\text { absorbance. } \\
\text { ** Due to the low volume and low biomass applied to ER4 bioreactors, crust } \\
\text { development was slow, and to increase the speed the bioreactors were re-inoculated after } \\
21 \text { days, which increased the rate of crust development significantly in the final } 10 \text { days } \\
\text { of bioreactor maintenance. }\end{array}$} \\
\hline
\end{tabular}




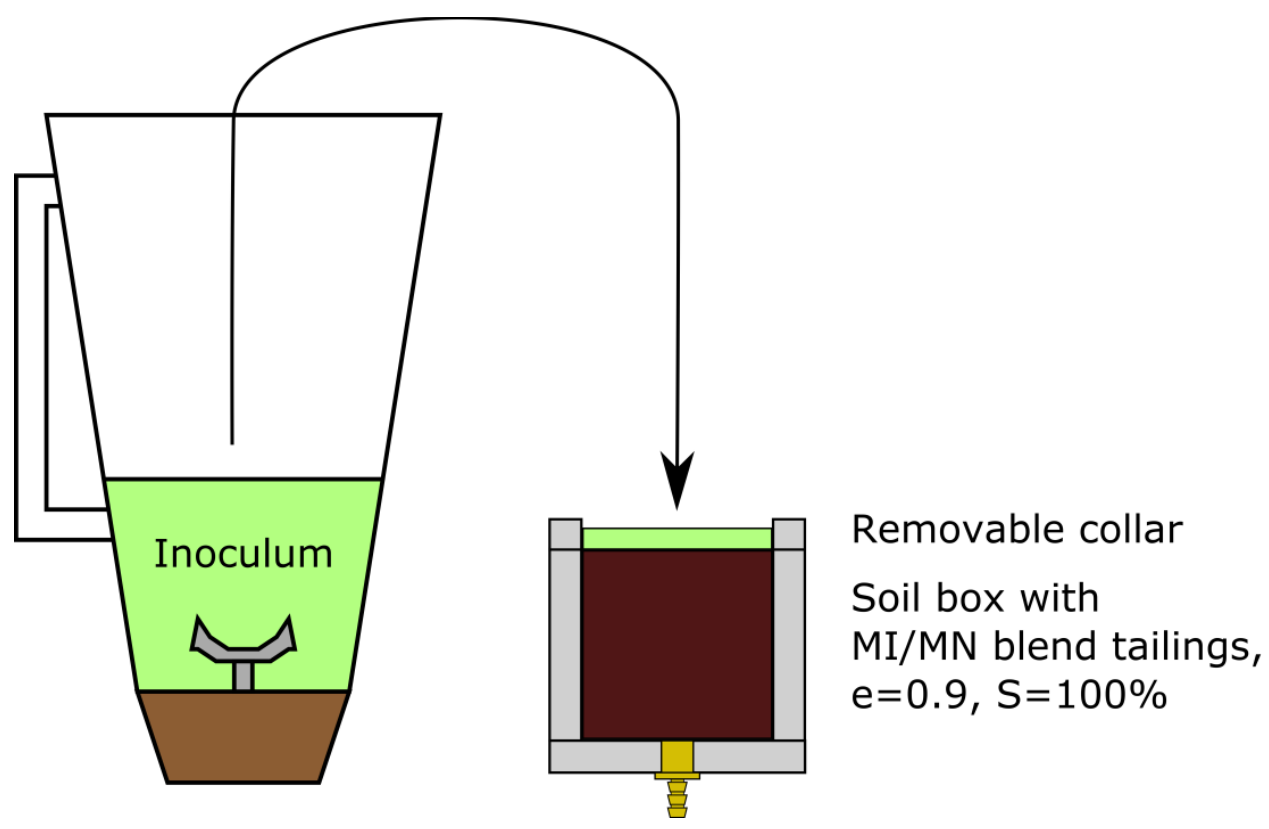

Figure 2-20 | Schematic of bioreactor inoculation, where one batch of $400 \mathrm{~mL}$ inoculum was divided evenly between 6 soil boxes, featuring removable collars to provide extra headspace in which the inoculum could reside until percolating through the tailings sample. 


\subsubsection{Biomass Characterization of Biological Soil Crusts}

To characterize the biomass present in each set of biological soil crusts being studied for wind erosion resistance, one bioreactor sample per set of six was sacrificed with destructive biomass testing. Samples of each crust were collected using syringe barrel soil coring and were oven dried. These dry samples were then used for additional analysis, including organic content by loss on ignition (LOI), polysaccharide content via phenol-sulfuric acid reaction assay, chlorophyll a content using spectrophotometry and trichromatic equation, and scanning electron microscopy. Details of these methods are described below.

The first step in biomass characterization was obtaining small sub-samples of the bioreactor, each containing the same amount of crust and underlying tailings for repeatability in analyses. This was accomplished via syringe barrel coring. A small syringe (10 mL BD syringe with Luer-Lok tip, cat 309604) with an inner diameter of 1.4 $\mathrm{cm}$ was used for coring. Given the dimensions of the syringe and the soil box, up to 16 cores could be extracted. To allow coring, the tip of the syringe was cut off using a hacksaw and smoothed using a file. Due to the high level of suction in the tailings, a vacuum had to be generated in the syringe for a sample to be extracted. Therefore, the syringe corer was inserted into the bioreactor to a depth of $0.65 \mathrm{~cm}$, with the syringe plunger in the $3 \mathrm{~mL}$ position. Next, the syringe plunger was pulled from the $3 \mathrm{~mL}$ to 6 $\mathrm{mL}$ mark (a distance of $1.95 \mathrm{~cm}$ ) to create suction, and then the syringe containing a core of crust and tailings was removed. The plunger was pushed through the syringe to extract the core, which was trimmed using a razor to a sample height of 0.1 " or 0.2 ", and placed into a sterile ceramic weighting dish. The cores were then dried at $110^{\circ} \mathrm{C}$ for 24 hours, and the dry weight was measured. This procedure, which has proven to be repeatable, yields samples that have a dry weight between 0.9 and 1.3 grams for the 0.1 " height cores and between 1.5 and 2.25 grams for the 0.2 " cores. These sample cores were used for all other analyses.

The total organic content of the biological soil crust samples was quantified by measuring LOI (ASTM D2974-14). For each set of biological soil crust samples, two 
0.2 " cores were used for organic content determination. Oven dried samples were placed into a muffle furnace at $440^{\circ} \mathrm{C}$ for 24 hours, removed from the muffle furnace and placed in a desiccator to cool, and then weighed. After weighing, the sample was reinserted into the muffle furnace for 1 hour at $440^{\circ} \mathrm{C}$, removed and cooled in a desiccator, and reweighed. This process was repeated until no change in sample mass was measured, indicating that all organics were burned off. The total change in sample mass from ignition was used to calculate total organic content of the sample, with duplicate results for each biological soil crust sample. This measurement served as one means of comparison between the biomass of the different biological soil crusts.

The polysaccharide content of the biological soil crust samples was used to measure EPS produced by the filamentous cyanobacteria. Measurements were made using the phenol-sulfuric acid reaction assay, as described by Dubois et al. (1956). To perform the phenol-sulfuric acid reaction, polysaccharides were separated from soil particles using methods presented by Colica et al. (2015).

For each set of biological soil crust samples, two 0.1 " cores were used for measurement of polysaccharides in each sample. From each core, 1 gram of dry sample was ground using a sterile mortar and pestle and placed into a $15 \mathrm{~mL}$ centrifuge tube (VWR $15 \mathrm{~mL}$ polypropylene centrifuge tube with conical-bottom, cat 89039). To each tube, $15 \mathrm{~mL}$ of $0.1 \mathrm{M} \mathrm{Na}_{2}$ EDTA was added, and the tube was mixed and then allowed to sit for 15 minutes at $25^{\circ} \mathrm{C}$. The tubes were centrifuged for 20 minutes at $3500 \mathrm{xg}(5440$ $\mathrm{rpm}$ ) to recover the polysaccharide extracts devoid of any soil particles in suspension. The supernatant was removed from the centrifuge tube and saved for analysis, while the cell/soil pellet was rehydrated with $15 \mathrm{~mL}$ of fresh $0.1 \mathrm{M} \mathrm{Na} 2$ EDTA, allowed to sit for 15 minutes at $25^{\circ} \mathrm{C}$, and then centrifuged again for 20 minutes at $3500 \mathrm{x}$ g. This process was repeated for a total of 4 extractions per tube. The supernatants from each tube and each extraction were stored at $4{ }^{\circ} \mathrm{C}$ before analysis. The phenol-sulfuric acid reaction was then carried out on these four supernatants from the two cores, in duplicate. First, test tubes were filled with $0.5 \mathrm{~mL}$ of supernatant, or, where dilutions were necessary to get absorbance readings within the range of the standard curve prepared, $0.25 \mathrm{~mL}$ of 
supernatant and $0.25 \mathrm{~mL}$ of distilled water were used. For the calibration curve, a blank was filled with $0.5 \mathrm{~mL}$ of distilled water, and glucose standards were filled with $0.5 \mathrm{~mL}$ of solutions containing 10 to $100 \mu \mathrm{g} / \mathrm{mL}$ of glucose (Figure 2-21). Next, $0.5 \mathrm{~mL}$ of phenol reagent ( $80 \%$ phenol solution by weight) was added to all tubes and mixed rapidly and thoroughly. Subsequently, $2.5 \mathrm{~mL}$ of reagent grade sulfuric acid was added to all tubes and mixed rapidly. The tubes were left to stand for 10 minutes, and then placed in a $25^{\circ} \mathrm{C}$ water bath for 15 minutes. The contents of each tube was transferred to a cuvette, and the absorbance measured at $488 \mathrm{~nm}\left(\mathrm{~A}_{488}\right)$ against the distilled water blank. The glucose standard curve, which was developed using at least three readings collected at each of six glucose concentration over the span of three months, was verified every time the assay was performed using a standard of $100 \mu \mathrm{g} / \mathrm{mL}$ of glucose. The standard curve (Figure 2-21) was used to determine polysaccharide concentration for each tube, and the polysaccharide concentration for each extraction was added to determine the total polysaccharide concentration for the sample. The concentration of polysaccharides measured in each sample, with quadruplicate results for each biological soil crust sample, served as an additional means of comparison between the biomass of the different biological soil crusts.

Chlorophyll a content was measured using a spectrophotometer and trichromatic equations, based primarily on the methods of Liu et al. (2008). For each set of biological soil crust samples, two 0.2 " cores were used for chlorophyll a determination. For each core, 2 grams of dry sample was ground using a sterile mortar and pestle and placed into a $15 \mathrm{~mL}$ centrifuge tube (VWR $15 \mathrm{~mL}$ polypropylene centrifuge tube with conicalbottom, cat 89039). To this tube, a 90\% acetone solution was added to reach a total volume of $10 \mathrm{~mL}$, and then $2.5 \mathrm{~mL}$ of DMSO was added. The tubes were refrigerated overnight in the dark at $4^{\circ} \mathrm{C}$, and then were centrifuged at $7000 \mathrm{rpm}$ for 10 minutes. The supernatant was separated, and analysis of each supernatant was done in duplicate. For each duplicate sample, $3 \mathrm{~mL}$ of supernatant was added to a cuvette, or, where dilutions were necessary to get absorbance readings within the range of the standard curve prepared, $1.5 \mathrm{~mL}$ of supernatant and $1.5 \mathrm{~mL}$ of $90 \%$ acetone was used. For each cuvette, 
the absorbance was measured at $384 \mathrm{~nm}, 490 \mathrm{~nm}$, and $663 \mathrm{~nm}$ against a $90 \%$ acetone blank using a Thermo Scientific Genesys 20 spectrophotometer. The trichromatic equations presented by Garcia-Pichel and Castenholz (1991) were then used to calculate the chlorophyll a concentration in units of absorbance/sample weight. The trichromatic equations used correct chlorophyll a absorbance for interferences caused by carotenoids and scytonemin. The potential interference of turbidity on chlorophyll a absorption was investigated in this study by measuring the absorbance of the samples at $750 \mathrm{~nm}$, and the effect of pheophytin a on chlorophyll a absorption was investigated by measuring the absorbance of the samples at $664 \mathrm{~nm}$ before acidification and $665 \mathrm{~nm} 90$ seconds after acidification with $0.1 \mathrm{~mL}$ of $0.1 \mathrm{~N} \mathrm{HCl}$ (American Public Health Association 1994). Based on these analyses, the effects of turbidity and pheophytin were found to be negligible for both the Eagle River crusts and Anabaena doliolum crusts. To convert to units of $\mu \mathrm{g}$ chl a/g sample, a standard chlorophyll a curve was developed by mixing 6 dilutions of a standard chlorophyll a solution, measuring the absorbance of the dilutions at $384 \mathrm{~nm}, 490 \mathrm{~nm}$, and $663 \mathrm{~nm}$, applying the trichromatic equations, and plotting against the known concentration of chlorophyll a in each dilution. The resulting chlorophyll a standard curve, which was only developed once, is shown in Figure 2-22. During the entire chlorophyll a extraction and measurement procedure, care was taken to keep samples cold $\left(4^{\circ} \mathrm{C}\right)$ and in the dark, as exposure to warm temperatures and light can cause degradation of extracted chlorophyll. The amount of chlorophyll a in each sample, determined with quadruplicate results through the use of the trichromatic equations and the chlorophyll a standard curve, served as an additional means of comparison between the biomass of the different biological soil crusts.

Lastly, environmental scanning electron microscopy (ESEM) was used to analyze the structure of the biological soil crusts and the interaction between the filamentous cyanobacteria and the tailings particles. For each set of biological soil crust samples, a small piece of crust was obtained from the crust surrounding the extracted cores, and was removed aseptically with microforceps and placed in a crucible. The crust was dried at $40^{\circ} \mathrm{C}$ for 3 days, so as not to negatively affect the crust organisms. This dried crust was 
then used to make the SEM mount. The mount was coated with 10 micron carbon coating (Buikema et al. 2015), and then the samples were observed using a FEI Philips XL 40 Environmental Scanning Electron Microscope. 


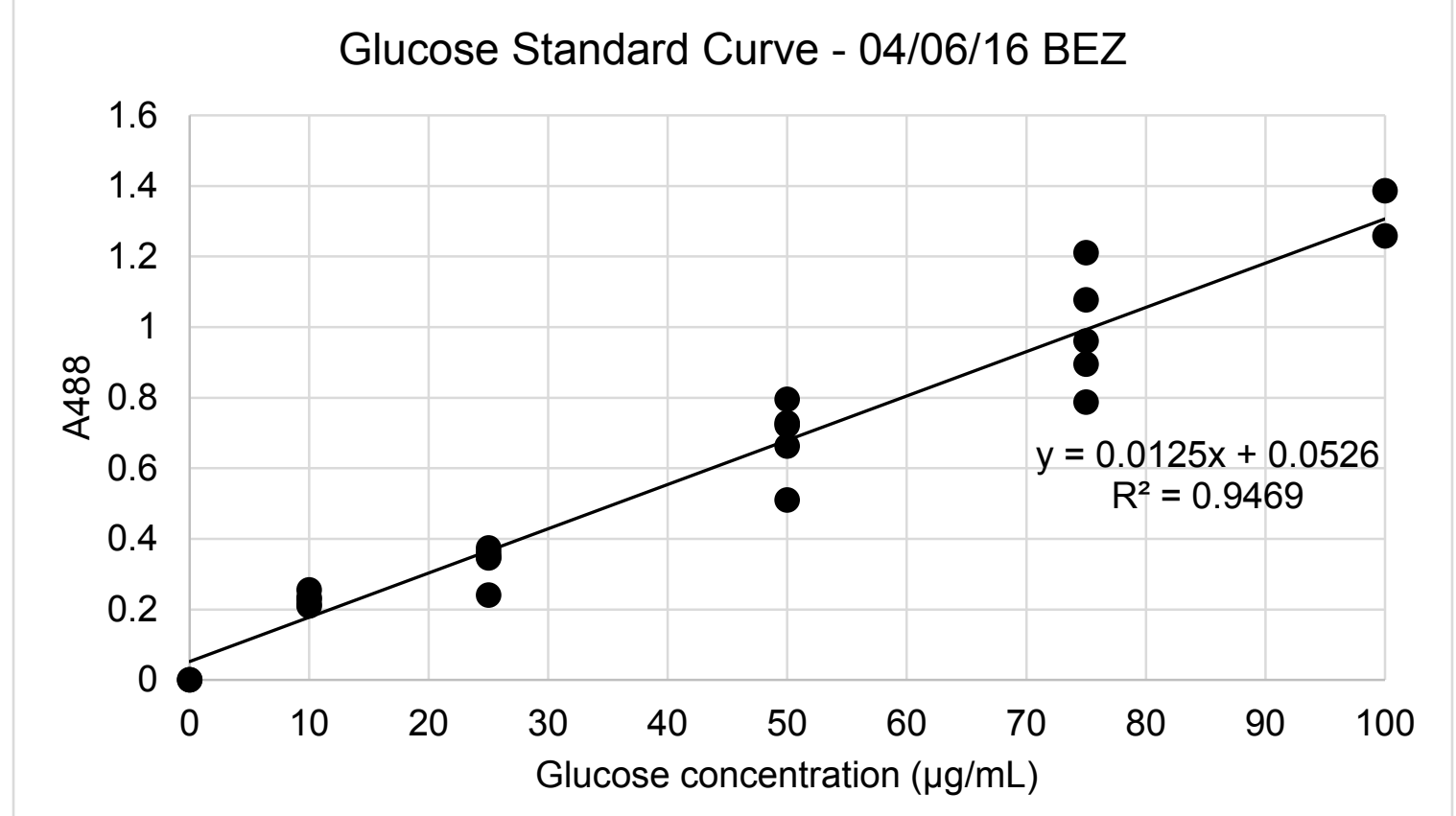

Figure 2-21 | Glucose standard curve used to determine polysaccharide concentration in biological soil crusts samples.

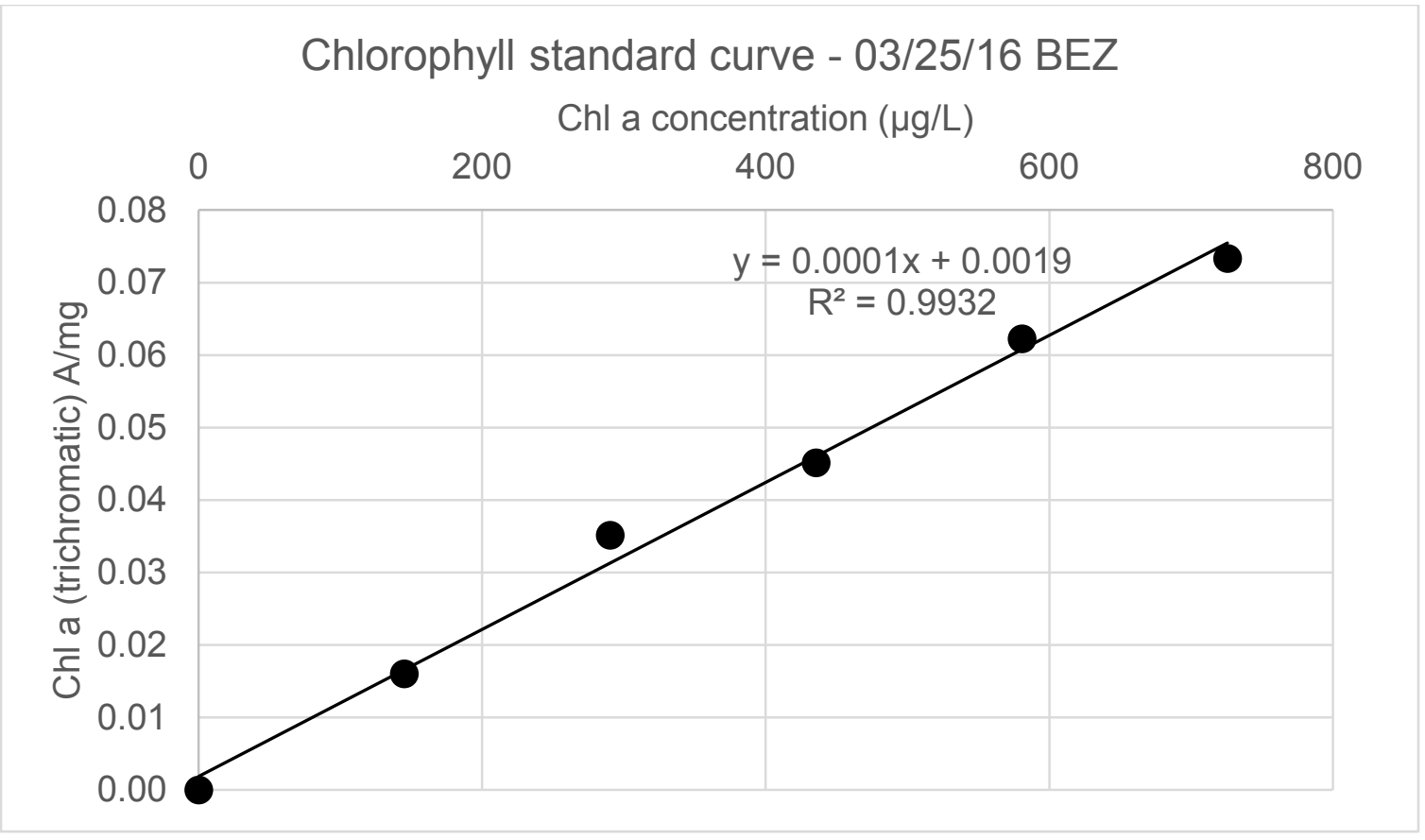

Figure 2-22 | Chlorophyll a standard curve used to determine chlorophyll a concentration in biological soil crusts samples. 


\subsubsection{Wind Erosion Resistance Testing of Biological Soil Crusts}

To characterize the wind erosion resistance provided by each type of biological soil crust studied, five bioreactors were used: three bioreactors for each crust were tested using the wind tunnel methods described in Section 2.4.2., and two bioreactors for each crust were tested using the ball drop methods described in Section 2.4.3. All five bioreactors for each crust type were exposed to 7 days of freezing/sublimation, as described in Section 2.4.1., prior to the wind tunnel and ball drop testing. The same test conditions used for the untreated tailings discussed in Section 2.4 were used for the biological soil crust samples so that a direct comparison could be made. For the wind tunnel testing, sample mass lost was the primary means of quantitative comparison between the wind erosion of different crust types and untreated tailings. Digital photographs of the samples before and after wind tunnel testing, and video recorded of the wind tunnel tests, were also used for qualitative analysis of the crust behavior in the wind tunnel. For the ball drop testing, impact crater diameter was the primary means of quantitative comparison between the resistance to impact provided by the different crust types and untreated tailings. 


\subsection{References}

Abrams, M., Hook, S., Ramachandran, B., and Center, E. D. (2002). "ASTER User Handbook Version 2." Jet Propulsion Laboratory, 4800.

Association, A. P. H. (1994). "Water Environment Federation (1998) Standard methods for the examination of water and wastewater." Washington, Dc.

ASTM B215-15, Standard Practices for Sampling Metal Powders, ASTM International, West Conshohocken, PA, 2015,www.astm.org.

ASTM C702-11, Standard Practice for Reducing Samples of Aggregate to Testing Size, ASTM International, West Conshohocken, PA, 2011, www.astm.org.

ASTM D422-63(2007), Standard Test Method for Particle-Size Analysis of Soils, ASTM International, West Conshohocken, PA, 2007, www.astm.org.

ASTM D2216-10, Standard Test Methods for Laboratory Determination of Water (Moisture) Content of Soil and Rock by Mass, ASTM International, West Conshohocken, PA, 2010, www.astm.org.

ASTM D2974-14, Standard Test Methods for Moisture, Ash, and Organic Matter of Peat and other Organic Soils, ASTM International, West Conshohocken, PA, 2014, www.astm.org.

ASTM D4648-16, Standard Test Methods for Laboratory Miniature Vane Shear Test for Saturated Fine-Grained Clayey Soil, ASTM International, West Conshohocken, PA, 2016, www.astm.org.

ASTM D5334-14, Standard Test Methods for Determination of Thermal conductivity of Soil and Soft Rock by Thermal Needle Probe Procedure, ASTM International, West Conshohocken, PA, 2014, www.astm.org.

ASTM D6938-15, Standard Test Methods for In-Place Density and Water Content of Soil and Soil-Aggregate by Nuclear Methods (Shallow Depth), ASTM International, West Conshohocken, PA, 2015, www.astm.org.

ASTM D7428-15, Standard Test Method for Resistance of Fine Aggregate to Degradation by Abrasion in the Micro-Deval Apparatus, ASTM International, West Conshohocken, PA, 2015, www.astm.org.

Buikema, N. D. (2015). "Stabilization of iron mine tailings through microbially induced calcite precipitation."

Buikema, N. D., Zwissler, B. E., Seagren, E. A., Oommen, T., and Vitton, S. J. (accepted). "Stabilization of Iron Mine Tailings through Biocalcification." Environmental Geotechnics.

Colica, G., Li, H., Rossi, F., De Philippis, R., and Liu, Y. (2015). "Differentiation of the characteristics of excreted extracellular polysaccharides reveals the heterogeneous primary succession of induced biological soil crusts." Journal of Applied Phycology, 27(5), 1935-1944.

De Philippis, R., Sili, C., Paperi, R., and Vincenzini, M. (2001). "Exopolysaccharideproducing cyanobacteria and their possible exploitation: a review." Journal of Applied Phycology, 13(4), 293-299. 
Dubois, M., Gilles, K. A., Hamilton, J. K., Rebers, P., and Smith, F. (1956). "Colorimetric method for determination of sugars and related substances." Analytical chemistry, 28(3), 350-356.

Garcia-Pichel, F., and Castenholz, R. W. (1991). "Characterization and Biological Implications of Scytonemin, a Cyanobacterial Sheath Pigment1." Journal of Phycology, 27(3), 395-409.

Gerhardt, P., Wood, W. A., and Krieg, N. R. (1994). Methods for general and molecular bacteriology, American Society for Microbiology Washington, DC.

Ghulam, A. (2009). "How to calculate reflectance and temperature using ASTER data." $<$ http://www.gis.slu.edu/RS/ASTER_Reflectance Temperature Calculation.php $>$

Han, Q., Qu, J., Zhang, K., Zu, R., Niu, Q., and Liao, K. (2009). "Wind tunnel investigation of the influence of surface moisture content on the entrainment and erosion of beach sand by wind using sands from tropical humid coastal southern China." Geomorphology, 104(3), 230-237.

Lindahl, V., and Bakken, L. R. (1995). "Evaluation of methods for extraction of bacteria from soil." FEMS Microbiology Ecology, 16(2), 135-142.

Liu, Y., Cockell, C. S., Wang, G., Hu, C., Chen, L., and De Philippis, R. (2008). "Control of Lunar and Martian dust-experimental insights from artificial and natural cyanobacterial and algal crusts in the desert of Inner Mongolia, China." Astrobiology, 8(1), 75-86.

McKenna Neuman, C., and Maxwell, C. (1999). "A wind tunnel study of the resilience of three fungal crusts to particle abrasion during aeolian sediment transport." Catena, 38(2), 151-173.

McKenna Neuman, C., and Maxwell, C. (2002). "Temporal aspects of the abrasion of microphytic crusts under grain impact." Earth Surf. Process. Landf., 27(8), 891908.

McKenna Neuman, C., Maxwell, C., and Rutledge, C. (2005). "Spatial and temporal analysis of crust deterioration under particle impact." Journal of Arid Environments, 60(2), 321-342.

McKenna Neuman, C., Maxwell, C. D., and Boulton, J. W. (1996). "Wind transport of sand surfaces crusted with photoautotrophic microorganisms." Catena, 27(3), 229-247.

O'Brien, P., and McKenna Neuman, C. (2012). "A wind tunnel study of particle kinematics during crust rupture and erosion." Geomorphology, 173, 149-160.

Observations, Y. C. f. E. (2012). "ASTER Images."

Okoli, R. E. (2003). "Wind tunnel study on aeolian saltation dynamics and mass flow." Journal of arid Environments, 53(4), 569-583.

Price, J. C., Vasher, D. A., Vitton, S. J., and Paterson, K. (1997). "Characterization of fugitive dust emissions from a northern Michigan iron mine." Tailings and Mine Waste '98, Balkema Publishers, Rotterdam, Netherlands, Fort Collins, CO, 743751. 
Stanier, R., Kunisawa, R., Mandel, M., and Cohen-Bazire, G. (1971). "Purification and properties of unicellular blue-green algae (order Chroococcales)." Bacteriological reviews, 35(2), 171.

Thenkabail, P. S., Lyon, J. G., and Huete, A. (2011). Hyperspectral remote sensing of vegetation, CRC Press.

Vitton, S. (1997). "Environmental and operational considerations in the use of paper waste sludge for dust control on iron ore mine tailings." First International Workshop on the Use of Paper Industry Sludges in Environmental Geotechnology and ConstructionHelsinki, Finland.

Vitton, S. J., and Muszynski, M. R. (2013). "Strength and Creep Properties of a Frozen Coastal Sand in Saltwater." Mechanical Properties of Frozen Soils, H. Zubeck, and Z. Yang, eds., ASTM International, West Conshohocken, PA, 153-166.

Waterbury, J. B. (2006). "The cyanobacteria—isolation, purification and identification." The prokaryotes, Springer, 1053-1073.

Zwissler, B. E., Vitton, S. J., Seagren, E. A., and Oommen, T. (in preparation). "Laboratory method to generate dust via sublimation to study cold weather dusting phenomenon for fine-grained sediments." 
Chapter 3 Thermal remote sensing for moisture content of mine tailings: laboratory study ${ }^{1}$

${ }^{1}$ The material contained in this chapter has been submitted to Environmental and Engineering Geoscience. 


\subsection{Introduction}

Massive volumes of waste materials are produced each year by mining operations. According to the U.S. Environmental Protection Agency (USEPA), between one and two billion metric tons of mine waste is produced annually in the U.S., excluding coal (USEPA 2014). Mine tailings result from ore beneficiation in which the crushing and grinding operations yield finely crushed rock particles (average particle size is $20 \mu \mathrm{m}$, with a significant portion between 1 and $10 \mu \mathrm{m}$ ) to separate the ore from the rock. Once produced, the tailings are often deposited in slurry form into permanent tailings impoundments (Vick 1983). Over 3500 active tailings impoundments exist worldwide (Davies et al. 2000). The impoundments constructed to contain tailings are among some of the largest earthen structures in the world, and are subject to intense regulatory and public attention because of the land areas they disturb and the hazards that can be associated with the mining waste they contain (Vick 1983).

One of the many important environmental hazards associated with tailings impoundments is air pollution from blowing dust (Buck and Gerard 2001; Stovern et al. 2014) (Figure 3-1a). Blowing dust due to wind erosion of mine tailings can negatively impact human activity via poor visibility for drivers and respiratory health problems (Bang et al. 2009). Blowing dust is regulated by the USEPA as airborne particulate matter based on particle size and particle concentration. Particles of concern include coarse particles (diameters 2.5-10 $\mu \mathrm{m}$ ) and fine particles (diameters less than $2.5 \mu \mathrm{m}$ ). The latter are of particular concern because they are small enough to reach the lower portions of the human respiratory tract and can cause respiratory health problems including damage to lung tissue, cancer, and premature death (USEPA 2012). Under the USEPA Clean Air Act, the current national air quality standard limits particles smaller than $2.5 \mu \mathrm{m}$ to $12 \mu \mathrm{g} / \mathrm{m}^{3}$ for an annual mean, or $35 \mu \mathrm{g} / \mathrm{m}^{3}$ for a 24-hour concentration, and limits particles $2.5-10 \mu \mathrm{m}$ to $150 \mu \mathrm{g} / \mathrm{m}^{3}$ for a 24-hour concentration (USEPA 2014). Dusting events at tailings impoundments can lead to violations of these regulations. Studying the concentration of particulate matter released from mine tailings impoundments is often desirable, especially if site conditions are favorable for dust 
generation and if the tailings impoundment is in close proximity to residential areas, as is the case for the Iron King Mine tailings impoundment, located in Dewey-Humboldt, AZ (Stovern et al. 2014).

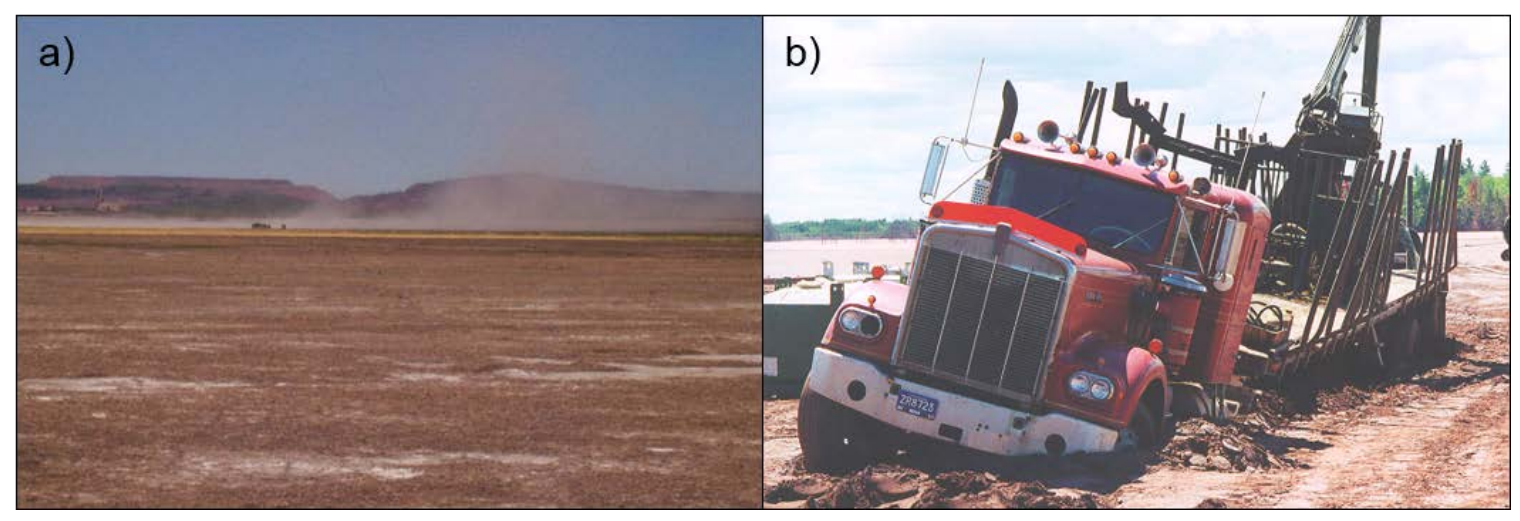

Figure 3-1 | Hazards associated with surface strength at mine tailings impoundments: (a) fugitive dust emissions, and (b) trafficability issues.

To control fugitive dust emissions, it is crucial to understand how the tailings are affected by wind erosion and how to detect when they may be susceptible to dusting. Typically, the lower the moisture content of the tailings is, the higher the dust susceptibility (Greeley and Iversen 1987; Nickling and Neuman 2009). However, even for well-studied tailings impoundments, it is difficult to make static and general characterizations about the tailings properties without considering spatial and temporal variations in the dynamic impoundment. Understanding how the moisture content changes spatially and temporally is complex, so monitoring the tailings to determine if and when certain regions of the impoundment may be susceptible to dusting is important. The traditional approach for monitoring dust emissions from tailings is reactive in nature rather than proactive, with a focus on collecting dust samples from gauges. This approach provides limited spatial coverage of the tailings, and is also costly, labor intensive, and affected by trafficability. Because of these limitations, the problem may not be detected before the dusting event occurs.

In addition to the actual hazard of blowing dust, trafficability can also be a concern for tailings impoundment managers, as shown in Figure 3-1(b) in which high surface moisture can result in mine equipment losing bearing capacity while applying dust 
control measures. Trafficability is also related to the surface moisture content of the tailings, and can be defined as the ability of the surface tailings to support the traffic of heavy equipment (Müller et al. 2011). Trafficabilty concerns include the ability of tailings to provide adequate traction for vehicles and the ability to resist excessive compaction and structural damage (Earl 1997; Paul and Vries 1979). While the hazard of fugitive dust emissions is caused by areas of tailings with low moisture content, accessing these regions for mitigation activities can be limited by regions of high moisture and low trafficability. Being able to identify regions of low trafficability is critical during planning and execution of dust mitigation operations.

The ever-changing surface conditions of any active tailings impoundment make it difficult to try to characterize the surface conditions of the tailings. For dust susceptibility and trafficability monitoring, remote sensing may be an effective method for monitoring the surface of tailings impoundments, because it provides a synoptic view of surficial processes with high spatial and temporal resolutions, making it useful for monitoring and detecting change. To utilize the high spatial and temporal resolution of remote sensing for monitoring the impoundment surface moisture content and strength, it is critical to derive the moisture content in the top layer of the tailings using remote sensing. In previous studies, thermal remote sensing has been used to (in) directly detect soil moisture (Liu and Zhao 2006; Minacapilli et al. 2012), but to the best of authors' knowledge no study has looked at its applicability for mine tailings. Therefore, it is hypothesized that if a relationship between the surface strength and moisture content of the tailings can be developed, then thermal remote sensing data can be indirectly related to strength, which is a critical factor for dust susceptibility as well as trafficability.

The objective of this study is to perform a preliminary laboratory-scale analysis to verify whether a relationship exists between moisture content and strength for the surface of mine tailings, as well as to verify whether thermal remote sensing can be used to derive the spatial variation in moisture content in surface tailings impoundments. Development of the laboratory-scale relationship between moisture content and strength required identification of one or more critical climatic variable(s) and evaluation of their 
influence on the remotely measured moisture content. Future efforts will scale the study to utilize high-spatial resolution thermal data collected with satellites or unmanned aerial vehicles (UAVs) in the field to detect changes in moisture content for the surface of tailings impoundments.

\subsection{Existing Techniques Utilizing Remote Sensing to Detect Moisture} Content

Thermal remote sensing relies on passively collecting thermal infrared electromagnetic energy that is emitted from objects in the 3-14 micrometer portion of the EMR spectrum. When this kinetic energy exits an object, it is converted to thermal infrared radiant energy, which can be measured using remote sensing (Price 1980). Being able to remotely detect an object's true kinetic temperature is a powerful tool: it enables the ability to know an object's temperature without having to measure it in-situ, and it also provides knowledge about the object's thermal properties. One such thermal property of interest is thermal inertia, which is a measure of a material or object's thermal response to temperature change. Thermal inertia varies with soil type and soil moisture content, and is commonly measured quantitatively for soils. Thermal remote sensing (particularly thermal inertia) has been shown to have a linear relationship with moisture content (Liu and Zhao 2006), and is a common method used to indirectly determine relative soil moisture (Minacapilli et al. 2012).

Numerous studies have used remote sensing to determine soil (apparent) thermal inertia (Murray and Verhoef 2007; Murray and Verhoef 2007; Putzig and Mellon 2007; Ramakrishnan et al. 2013; Soliman et al. 2013; Wang et al. 2010; Xue and Cracknell 1995). Cracknell and Xue (1996) provide a thorough review of the early work performed on satellite-based mapping of thermal inertia, but airborne (Maltese et al. 2013) and ground-based remote sensing systems (Liu and Zhao 2006) have also been used. Many studies have also used remotely sensed (apparent) thermal inertia to indirectly determine soil moisture (Cai et al. 2005; Cai et al. 2007; Kahle et al. 1984; Liu and Zhao 2006; Minacapilli et al. 2012; Price 1980; Price 1985; Scheidt et al. 2010; Soliman et al. 2013; Zhang et al. 2002). 
There is significant evidence in the literature that thermal remote sensing can be related to soil moisture. However, the authors found no studies investigating the specific application to mine tailings. If anything, remote sensing of tailings impoundments should be easier to implement than remote sensing of other soil applications, because the lack of vegetation on the impoundment makes it possible to sense soil surface conditions, which is often a limitation to using remote sensing for soil. In addition, the tailings contained in a given impoundment have exceptional uniformity when compared to most other heterogeneous soils and are mineralogically similar, so the only real variables within an impoundment is tailings grain size distribution and moisture content. While this study aims to fill that gap and to detect the moisture content of mine tailings using thermal remote sensing, it is worth noting that soil moisture can also be sensed using other types of remote sensing (Petropoulos et al. 2015), most commonly RADAR (Anderson and Croft 2009; Bindlish et al. 2006; Bindlish et al. 2003; Jackson 1997). However, the data processing for RADAR remote sensing is much more intensive than that for thermal remote sensing (Jensen 2009), so this study focused on utilizing the simpler and more readily available thermal remote sensing.

Before performing this study, the authors conducted preliminary laboratory tests with mine tailings that showed remote sensing for (apparent) thermal inertia was not an appropriate method for capturing variations in moisture content of the tailings. Rather than trying to modify the methods used by other studies (as described above) that were often developed for use with a specific satellite or sensor, this research tried to identify the critical remote sensing and atmospheric variables that allowed for variations in moisture content of surface tailings to be detected; in this way, these variables could be obtained and used for remote sensing data collected from any platform (e.g., laboratory, UAV, or satellite) and at any scale (e.g., laboratory- or field-scale).

\subsection{Laboratory Methods}

The laboratory testing was conducted using tailings samples collected from two North American iron mines: magnetite tailings from an impoundment at a mine in Michigan (these tailings will be referred to as MI-magnetite tailings in this paper, or MI-mag for 
short), and magnetite tailings from an impoundment at a mine in Minnesota (these tailings will be referred to as $M N$-magnetite tailings, or $M N$-mag for short, in this paper). These samples of magnetite tailings used have a specific gravity of 3.09 (MI-mag) and 2.91 (MN-mag), as determined using a Micromeritics AccuPyc 1330 Helium Pycnometer. The MI-mag and MN-mag tailings were determined to be 95 percent and 82 percent silt-sized particles and 5 percent and 6 percent clay-sized particles, respectively, based on hydrometer analysis following ASTM D422-63 (2007). These values are similar to those found during laboratory characterization of other mine tailings samples (Qiu and Sego 2001). All laboratory samples were prepared as much as possible to mimic the actual conditions in the tailings impoundment. Tailings are deposited into the impoundments in a 55 percent solid/45 percent liquid slurry (Muszynski 2000; Price 1998; Vasher 1999), and gradually dry from fully saturated conditions; for the MI-mag tailings, surface moisture contents have been measured in-situ in the top $2 \mathrm{~cm}$ of tailings from 5 percent to 30 percent by mass (ASTM D2216-10), and surface void ratios have been measured in-situ in the top $15 \mathrm{~cm}$ of tailings to range between 0.7 and 1.2 , with a mean of 0.9 (Price 1998). Therefore, all laboratory samples were prepared at void ratios between 0.8 and 1.0 in $76 \mathrm{~mm} \times 76 \mathrm{~mm} \times 76 \mathrm{~mm}$ polycarbonate soil boxes and fully saturated using a Mariotte tube constant head device. Even though field tailings deposition occurs via wet pluviation and leads to particle size sorting across the impoundment, the authors chose to constitute samples using dry pluviation followed by saturation for sample repeatability, and do not believe that significant variation from field conditions exist in these laboratory specimens due to their small size.

Once the MI-mag and MN-mag tailings samples were prepared to fully saturated conditions, they were exposed to an artificial diurnal heating cycle daily, and were monitored and tested until the samples were dry. This approach provided a range of moisture/strength conditions per sample over the duration of testing. Diurnal heating cycles were simulated in the laboratory by heating samples for 6 hours daily under two 500-Watt halogen lamps and two full spectrum lights mounted at a $45^{\circ}$ angle to the samples at a height of $0.7 \mathrm{~m}$ above the samples. Only the surface of the samples was 
exposed to the lamp. A schematic of the test setup is shown in Figure 3-2. A suite of measurements were taken twice daily for samples - once before heating, called "preheating", and once after heating, called "post-heating. These measurements are outlined in Table 3-1, and are described in more detail below.

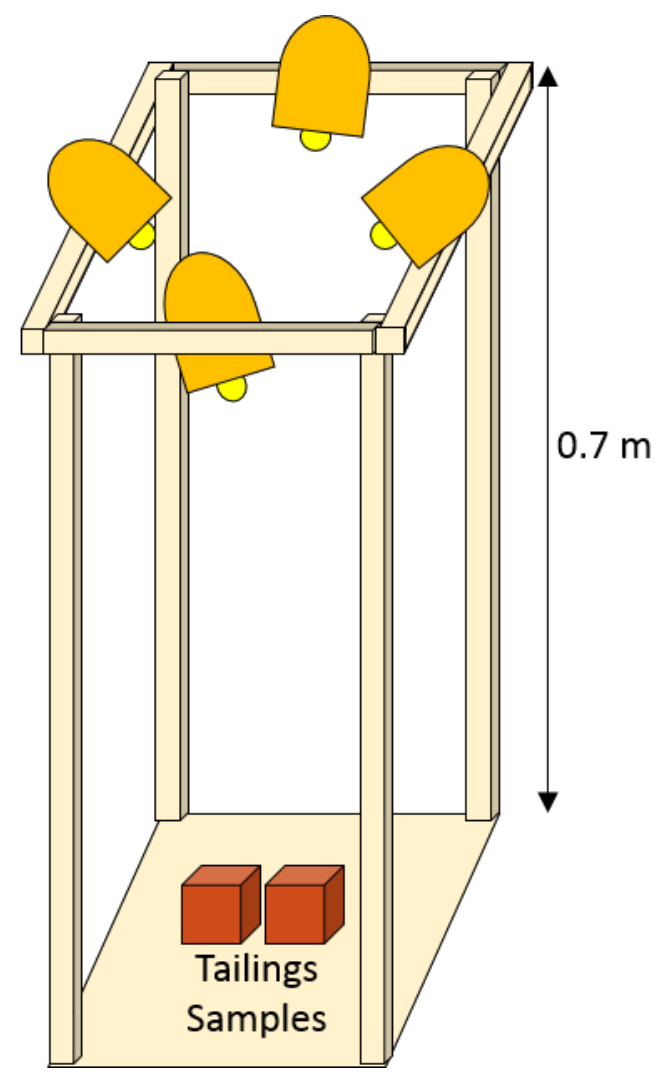

Figure 3-2 | Schematic of laboratory thermal remote sensing testing set-up, featuring two halogen lamps and 2 full-spectrum lamps mounted at 45 degree angles from iron samples. 
Table 3-1 | Details of thermal remote sensing laboratory testing conducted per sampling event.

\begin{tabular}{lllc}
\hline \multicolumn{1}{c}{$\begin{array}{c}\text { Measurement } \\
\text { Collected }\end{array}$} & \multicolumn{1}{c}{ Instrumentation } & Purpose of Measurement & $\begin{array}{c}\text { Tests } \\
\text { per } \\
\text { Sample }\end{array}$ \\
\hline Mass (g) & AND EK Balance & $\begin{array}{l}\text { Moisture content } \\
\text { calculation }\end{array}$ & 1 \\
\hline $\begin{array}{l}\text { Penetration depth } \\
(\mathrm{cm})\end{array}$ & $\begin{array}{l}\text { Humboldt H-1200 } \\
\text { needle penetrometer }\end{array}$ & $\begin{array}{l}\text { Represent relative surface } \\
\text { strength }\end{array}$ & 5 \\
\hline $\begin{array}{l}\text { Spectral reflectance } \\
(\%)\end{array}$ & $\begin{array}{l}\text { FieldSpec HandHeld } \\
\text { Pro Spectroradiometer } \\
\text { with plant probe }\end{array}$ & Albedo calculation & 10 \\
\hline $\begin{array}{l}\text { Atmospheric temp. } \\
(\mathrm{K}) \text { \& humidity }(\%)\end{array}$ & $\begin{array}{l}\text { General Tools PTH8708 } \\
\text { Thermohygrometer }\end{array}$ & $\begin{array}{l}\text { Quantify atmospheric } \\
\text { variation }\end{array}$ & 1 \\
\hline Thermal image $(\mathrm{K})$ & $\begin{array}{l}\text { FLIR SC640 thermal } \\
\text { camera }\end{array}$ & $\begin{array}{l}\text { Quantify surface temp \& } \\
\Delta \text { T due to diurnal heating }\end{array}$ & 1 \\
\hline
\end{tabular}

Gravimetric moisture content was determined by monitoring sample mass loss on a balance. The sample mass measured at a given sampling event was subtracted from the initial sample mass recorded during sample preparation to determine the mass of water lost, which was used to back-calculate moisture content. The reported moisture content is the average moisture content for the entire $76 \mathrm{~mm} \times 76 \mathrm{~mm} \times 76 \mathrm{~mm}$ sample, and it is worth noting that the surface moisture content conditions are likely lower than the bulk moisture content. However, because the samples started at the same conditions and were exposed to the same heating conditions, it is expected that all samples dried in the same way, and therefore the bulk moisture content should have a direct relationship with surface moisture content. For ease of data collection, bulk moisture content was measured rather than surface moisture content. Relative soil strength was quantified using the penetration depth $(\mathrm{cm})$ from a Humboldt $\mathrm{H}-1240$ universal needle penetrometer with Humboldt H-1280 40-45 mm standard hardened stainless steel needle, 150 g drop weight, and drop duration of 0.5 seconds. Five measurements were taken at each sampling event, one in each quadrant of the soil box and one in the center, with no less than $3 \mathrm{~cm}$ between penetration positions. The penetration depth measurements were then averaged to yield an average penetration depth, which was used to represent the relative surface strength of 
each sample. Surface albedo was measured using an ASD FieldSpec ${ }^{\circledR}$ HandHeld Pro $(325-1075 \mathrm{~nm})$ with plant probe; the average of 10 spectral reflectance curves per sample was used to calculate albedo as the mean reflectance from 400 to $1075 \mathrm{~nm}$. Albedo was obtained to try to quantify the color change associated with change in moisture of the tailings. Atmospheric temperature (K) and humidity (percent) were measured using a thermohygrometer pre-heating and post-heating. Change in surface temperature $(\mathrm{K})$ was measured using a FLIR ThermaCAM SC640 thermal camera. For the thermal camera to measure surface temperature rather than radiant energy, FLIR object parameters were measured as described in Table 3-2; the surface temperature of each sample, which was used to calculate the change in surface temperature between the diurnal heating cycles, was determined using FLIR ThermaCAM Researcher software. The magnitude of soil temperature change, with respect to atmospheric temperature change, is a function of soil moisture. This is why the authors looked at change in temperature due to diurnal heating cycles, rather than just an average daily temperature or an instantaneous temperature. Figure 3-3 contains thermal images collected for a MN-mag sample before and after heating, which was used to calculate change in sample temperature (for these images, change in sample temperature was $11.7 \mathrm{~K}$ ).

Testing of the MI-mag tailings occurred during the summer of 2014, while testing of the MN-mag tailings occurred in the summer of 2015. Between two and four samples were tested at a time, with one data point collected per day per sample, yielding 45 total data points for the MI-mag tailings and 48 total data points for the MN-mag tailings. After all data were collected, the relationships between the measured variables for each set of tailings were explored using regression analysis. 
Table 3-2 | Description of each FLIR object parameter and how the parameter was quantified.

\begin{tabular}{ll}
\hline \multicolumn{1}{c}{ Object Parameter } & \multicolumn{1}{c}{ How to Quantify Each Parameter } \\
\hline Emissivity & $\begin{array}{l}\text { Calculated as complement of albedo from } \\
\text { spectroradiometer }\end{array}$ \\
\hline Reflected apparent temperature $(\mathrm{K})$ & Same value as atm. temperature \\
\hline Object distance $(\mathrm{m})$ & $\begin{array}{l}\text { 0.7 meters (dist. between sample surface and camera } \\
\text { lens) }\end{array}$ \\
\hline Atmospheric temperature (K) & $\begin{array}{l}\text { Measured with thermohygrometer between sample } \\
\text { and lens }\end{array}$ \\
\hline Relative humidity $(\%)$ & $\begin{array}{l}\text { Measured with thermohygrometer between sample } \\
\text { and lens }\end{array}$ \\
\hline $\begin{array}{l}\text { External optics temperature }(\mathrm{K}) \text { and } \\
\text { transmission }\end{array}$ & $\begin{array}{l}\text { 1.0 (value to be used when no external optics for } \\
\text { camera) }\end{array}$ \\
\hline
\end{tabular}

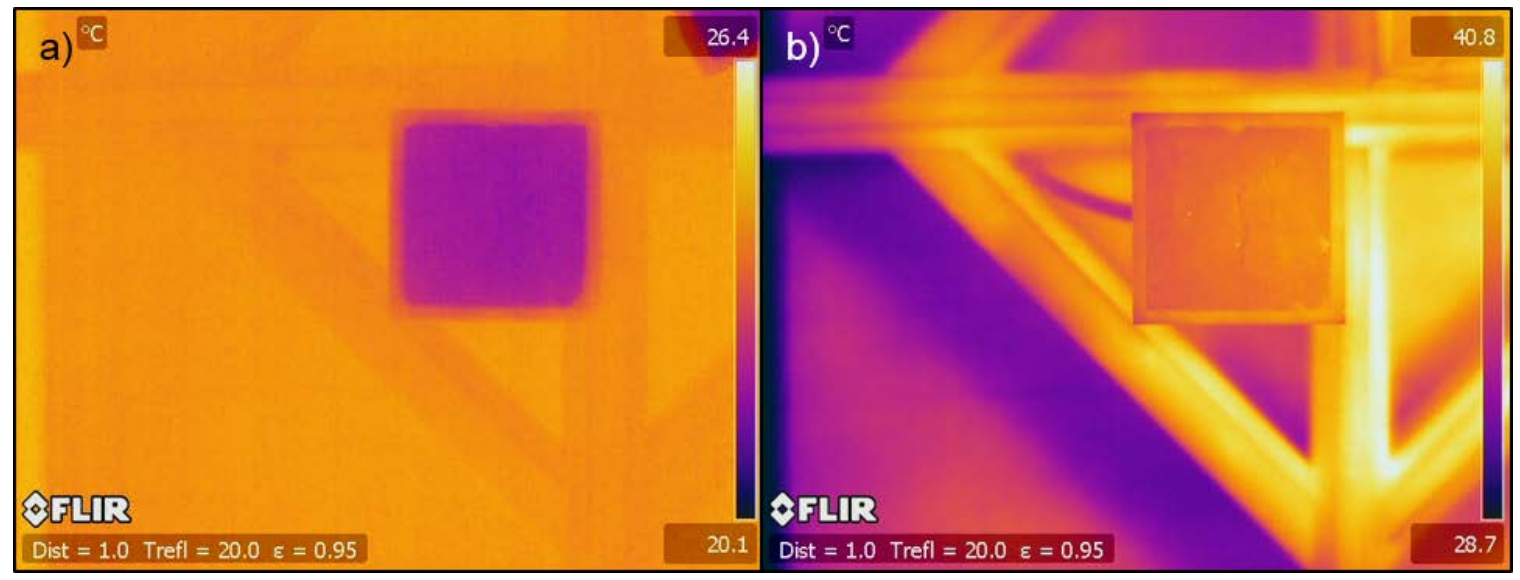

Figure 3-3 | Thermal images collected for a MN-mag sample on June 8, 2015: (a) preheating, with a measured sample moisture content of 8.6 percent, a measured sample penetration depth of $0.40 \mathrm{~cm}$, and an average sample temperature of $295.1 \mathrm{~K}$; and (b) post-heating, with a measured sample moisture content of 7.3 percent, a measured sample penetration depth of $0.35 \mathrm{~cm}$, and an average sample temperature of $306.8 \mathrm{~K}$.

\subsection{Laboratory Results}

As stated previously, it was hypothesized that if a relationship between the surface strength and moisture content of the iron tailings exists, then it can be used to indirectly relate thermal remote sensing data to the strength/dusting susceptibility of the tailings. To validate this hypothesis, the first relationship explored was that between moisture content and strength for the iron tailings. 
The relationship between daily average moisture content and penetration depth, which is used to represent surface strength, is shown in Figure 3-4 for the MI-mag tailings and Figure 3-5 for the MN-mag tailings. Penetration depth has an inverse relationship with surface strength, because a stronger surface means lower penetration depth. The relationship between moisture content and penetration depth is not the same for the MI-mag and MN-mag tailings, demonstrating the need to consider site-specific differences between tailings samples. Nevertheless, the overall shape of the curves is similar, which indicates that it is reasonable to relate moisture content and strength for mine tailings. Gravimetric moisture content $(\mathrm{w})$ can be used to predict penetration depth for MI-mag tailings using the following relationship:

$$
\text { Penetration Depth }(\mathrm{cm})=0.1996 \mathrm{e}^{0.1087 w}
$$

where the $\mathrm{R}^{2}$ is 0.76 and the RMSE is $0.06 \mathrm{~cm}$. Similarly, moisture content can be used to predict penetration depth for $\mathrm{MN}$-mag tailings using the following relationship:

$$
\text { Penetration Depth }(\mathrm{cm})=0.2072 \mathrm{e}^{0.0718 \mathrm{w}}
$$

where the $\mathrm{R}^{2}$ is 0.84 and the RMSE is $0.04 \mathrm{~cm}$. These relationships validate that any relationship that uses thermal remote sensing to predict moisture content can be indirectly applied to determine the relative surface strength of mine tailings, as long as the relationship between moisture content and strength is investigated for each new tailings sample being considered. For instance, the moisture content range for these two samples differs (approximately 0 to 20 percent for MI-mag, and 0 to 30 percent for MN-mag) for the same strength range (penetration depths of approximately 0 to $2 \mathrm{~cm}$ ), which is assumed to capture strength variations that would be needed to detect trafficability and dust susceptibility issues. 


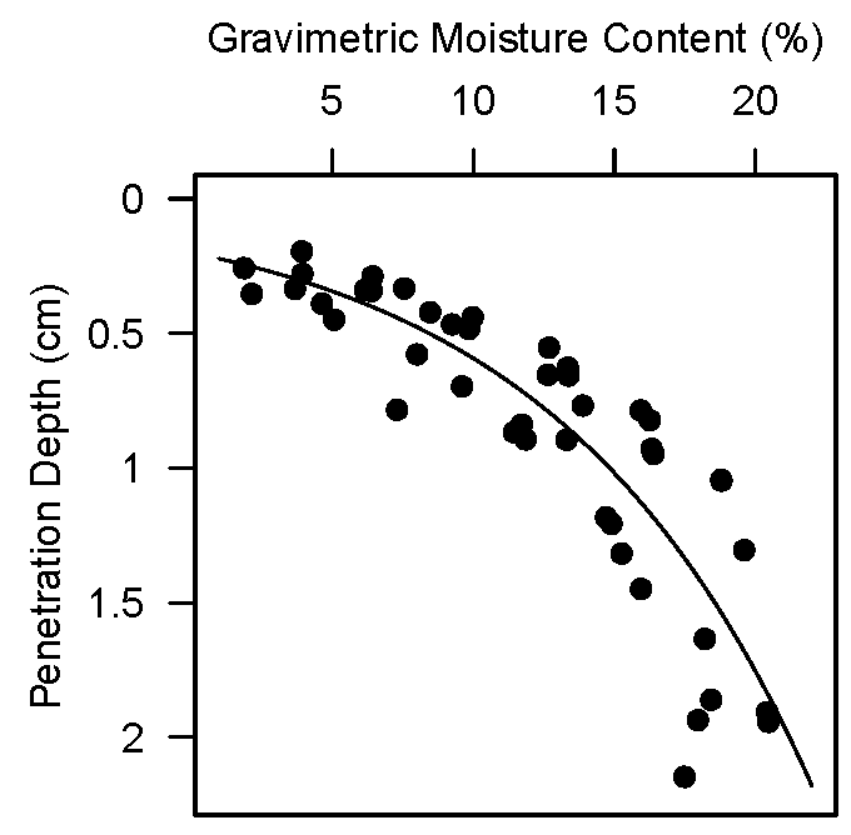

Figure 3-4 | Relationship between moisture content and surface strength (penetration depth) for MI-mag tailings. Each data point represents the daily average measurement for a sample.

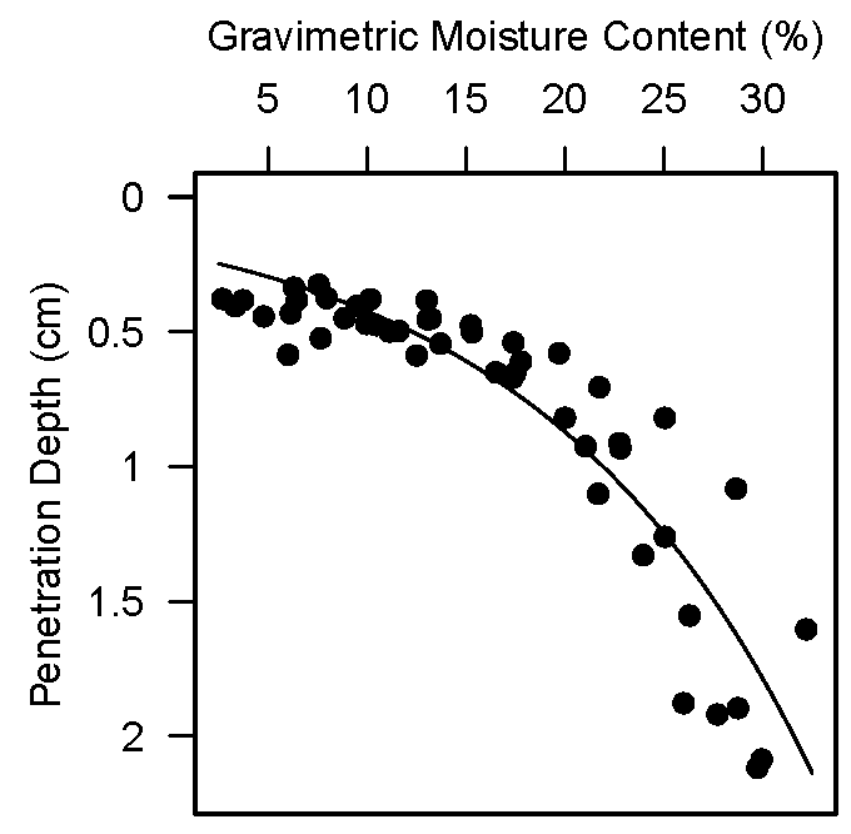

Figure 3-5 | Relationship between moisture content and surface strength (penetration depth) for MN-mag tailings. Each data point represents the daily average measurement for a sample. 
Once it was validated that a relationship exists between moisture content and strength for mine tailings, the relationship between the thermal remote sensing variables and moisture content, the dependent variable, was explored. Table 3-3 contains statistical information for each variable considered based on the laboratory data collected for the MI-mag tailings, and Table 3-4 contains the same information for the MN-mag tailings. Albedo was considered as a way to quantify the color change associated with change in moisture of the tailings. Sample temperature was directly determined with thermal remote sensing. While atmospheric temperature and atmospheric humidity were inputs for the thermal camera used to detect sample temperature, the trends in atmospheric conditions were also considered. The atmospheric temperature and humidity during laboratory testing was monitored, and the trend is shown in Figure 3-6 for the MI-mag testing and Figure 3-7 for the MN-mag testing. These data reveal that the atmospheric conditions of atmospheric temperature and relative humidity varied widely throughout the testing periods. Furthermore, there was no clear relationship between the variation in temperature/humidity in the pre- and post-heating measurements. These variations reflected in the data can be explained by the fact that testing was performed during the summer in a laboratory that was not air conditioned and did not have any means for temperature/humidity control. These laboratory data were used to develop a multivariate linear regression to relate thermal remote sensing variables and atmospheric variables to the moisture content of the iron tailings, which is discussed in the following section. 
Table 3-3 | Statistical information for each variable considered in relationship between moisture content and thermal remote sensing for MI-mag tailings, $\mathrm{n}=45$.

\begin{tabular}{lcccccc}
\hline \multicolumn{1}{c}{ Variable (units) } & Symbol & Mean & Median & Min. & Max. & $\begin{array}{c}\text { Std. } \\
\text { Dev. }\end{array}$ \\
\hline Albedo & $\alpha$ & 0.0642 & 0.0626 & 0.0535 & 0.0812 & 0.0069 \\
Pre-heating atmospheric temp. (K) & $\mathrm{T}_{\text {atm,pre }}$ & 297.6 & 297.6 & 294.6 & 300.0 & 1.6381 \\
Post-heating atmospheric temp. (K) & $\mathrm{T}_{\text {atm,post }}$ & 320.7 & 320.2 & 314.0 & 325.4 & 3.0731 \\
Change in atmospheric temp. (K) & $\Delta \mathrm{T}_{\text {atm }}$ & 23.13 & 23.10 & 18.30 & 26.20 & 1.9976 \\
Pre-heating atm. humidity (\%) & $\mathrm{H}_{\text {pre }}$ & 49.58 & 47.00 & 31.00 & 64.00 & 9.9190 \\
Post-heating atm. humidity (\%) & $\mathrm{H}_{\text {post }}$ & 20.33 & 21.00 & 13.00 & 28.00 & 4.3432 \\
Change in atm. humidity (\%) & $\Delta \mathrm{H}$ & 29.24 & 29.00 & 16.00 & 42.00 & 6.8694 \\
Pre-heating sample temp. (K) & $\mathrm{T}_{\text {samp,pre }}$ & 295.7 & 295.6 & 293.2 & 298.2 & 1.6385 \\
Post-heating sample temp. (K) & $\mathrm{T}_{\text {samp,post }}$ & 307.2 & 307.0 & 302.4 & 316.2 & 2.5696 \\
Change in sample temp. (K) & $\Delta \mathrm{T}_{\text {samp }}$ & 11.48 & 11.00 & 7.10 & 18.80 & 2.2554 \\
Gravimetric moisture content (\%) & $\mathrm{w}$ & 11.87 & 12.64 & 1.89 & 20.45 & 5.3353
\end{tabular}

Table 3-4 | Statistical information for each variable considered in relationship between moisture content and thermal remote sensing for $M N$-mag tailings, $\mathrm{n}=48$.

\begin{tabular}{lcccccc}
\hline \multicolumn{1}{c}{ Variable (units) } & Symbol & Mean & Median & Min. & Max. & $\begin{array}{c}\text { Std. } \\
\text { Dev. }\end{array}$ \\
\hline Albedo & $\alpha$ & - & - & - & - & - \\
Pre-heating atmospheric temp. (K) & $\mathrm{T}_{\text {atm,pre }}$ & 295.9 & 295.9 & 294.4 & 297.5 & 0.9544 \\
Post-heating atmospheric temp. (K) & $\mathrm{T}_{\text {atm,post }}$ & 311.7 & 313.0 & 299.4 & 327.2 & 7.4722 \\
Change in atmospheric temp. (K) & $\Delta \mathrm{T}_{\text {atm }}$ & 15.86 & 17.25 & 4.80 & 30.26 & 7.3901 \\
Pre-heating atm. humidity (\%) & $\mathrm{H}_{\text {pre }}$ & 40.23 & 40.80 & 30.80 & 53.50 & 6.0573 \\
Post-heating atm. humidity (\%) & $\mathrm{H}_{\text {post }}$ & 22.42 & 22.25 & 11.80 & 35.80 & 6.0324 \\
Change in atmospheric humidity (\%) & $\Delta \mathrm{H}$ & 17.81 & 17.00 & 9.80 & 27.80 & 4.4687 \\
Pre-heating sample temperature (K) & $\mathrm{T}_{\text {samp,pre }}$ & 294.0 & 294.4 & 290.4 & 297.2 & 1.6079 \\
Post-heating sample temperature (K) & $\mathrm{T}_{\text {samp,post }}$ & 306.2 & 305.6 & 302.0 & 312.6 & 2.6766 \\
Change in sample temperature (K) & $\Delta \mathrm{T}_{\text {samp }}$ & 12.24 & 12.00 & 9.50 & 17.30 & 1.7649 \\
Gravimetric moisture content (\%) & $\mathrm{w}$ & 16.04 & 15.28 & 2.67 & 32.20 & 8.3110
\end{tabular}




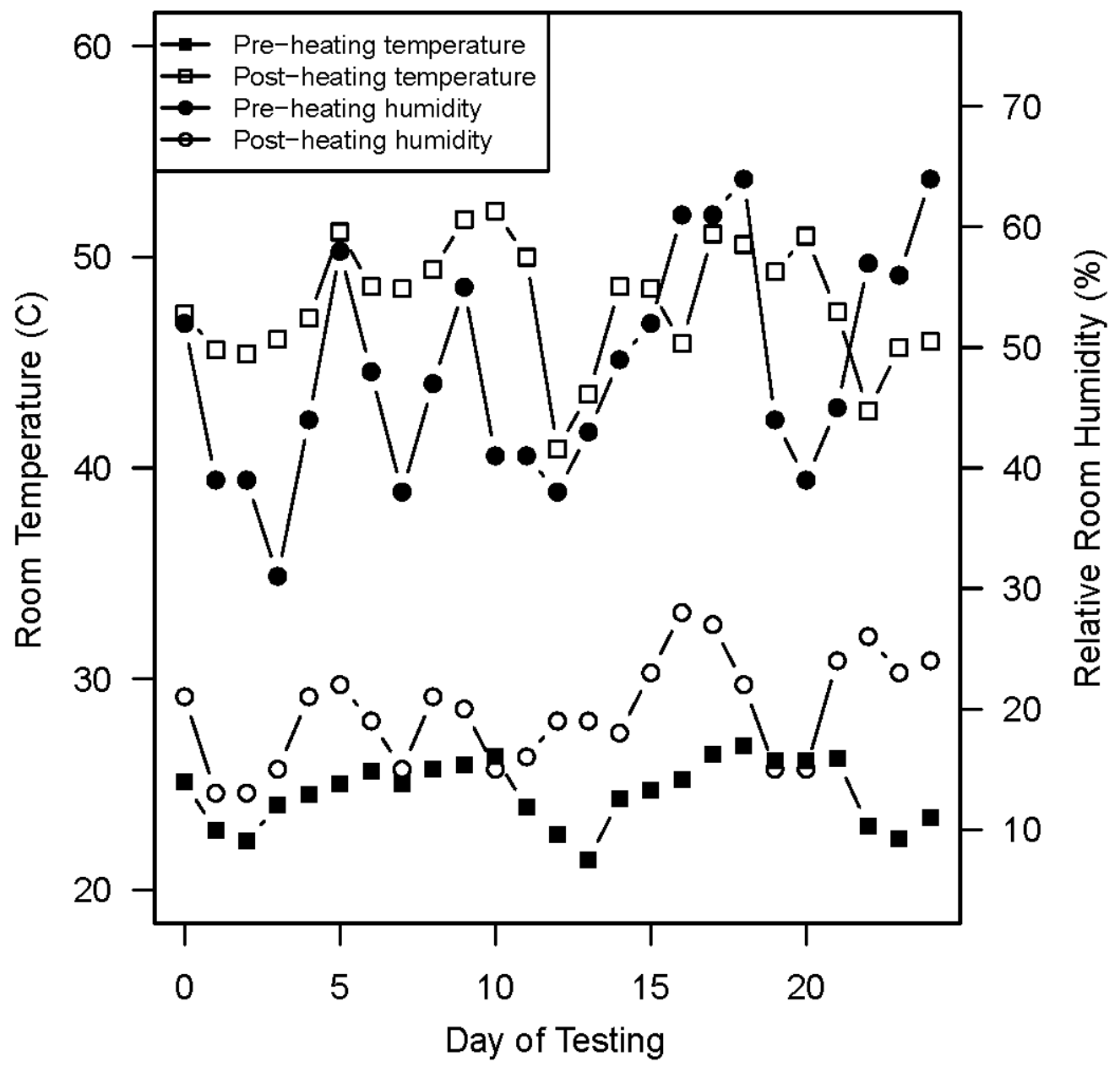

Figure 3-6 | Atmospheric conditions during laboratory testing of MI-mag tailings. Each data point represents a single measurement. 


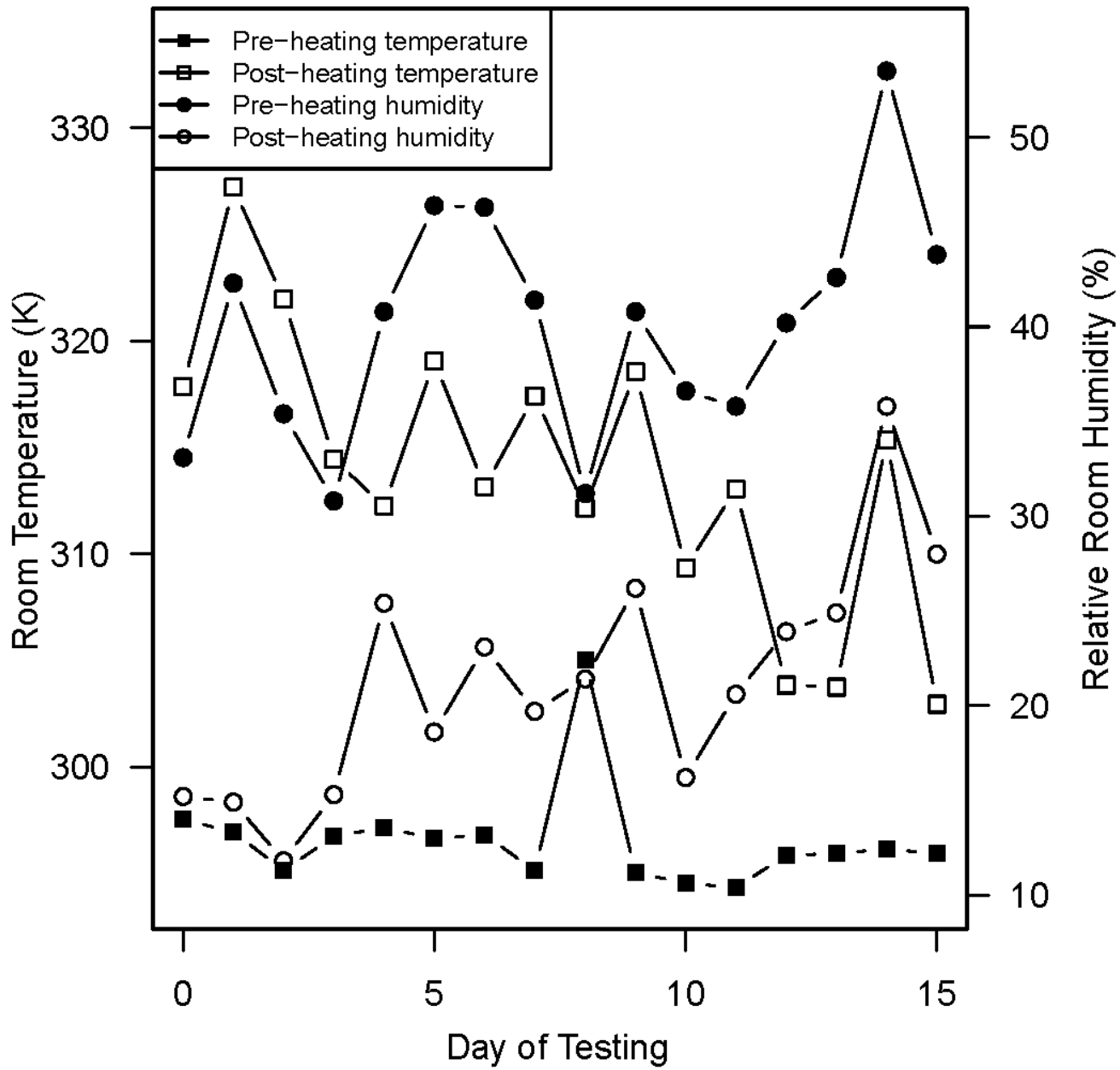

Figure 3-7 | Atmospheric conditions during laboratory testing of MN-mag tailings. Each data point represents a single measurement.

\subsection{Multivariate Regression Model Results and Discussion}

For each set of tailings, a multivariate regression was developed using sample temperature, sample albedo, and atmospheric conditions in an attempt to predict the moisture content of the MI-mag and MN-mag tailings using thermal remote sensing variables. The program RStudio (R Core Team 2014; R Studio Team 2015) was used to explore the relationships between the variables, to determine a multivariate linear regression for moisture content, and to perform statistical analysis of the regression 
model. The multivariate linear regressions were developed using the dataset of 45 data points (Table 3-3) for the MI-mag tailings and 48 data points (Table 3-4) for the MN-mag tailings.

The development of a multivariate linear regression to predict the moisture content of the tailings was carried out in the same way for both sets of tailings. Rather than using advanced machine-learning techniques, a manual, iterative approach was used to assess different combinations of variables so that the significance of the variables selected, individually and in combination, could be assessed. Statistical tools were used to assess the validity of the regressions and the significance of the variables used. The validity of the regressions was assessed using tools such as root mean squared error (RMSE) and coefficient of determination $\left(\mathrm{R}^{2}\right)$. The significance of the contribution of the variables was assessed using tools such as adjusted $\mathrm{R}^{2}$ and regression parameter hypothesis test $(\mathrm{t}$ test). Statistical tools, like Q-Q plots and histograms, were used to identify variables that could benefit from transformation. The original variables, as well as multiple transformations (e.g., log- and square root transformations) were tested using the same process to assess which transformation, if any, added the most value to the regression. A more complete description of the regression development technique can be found in Zwissler et al. (2014). For brevity, only the final regression equation that provided the best statistical performance for each set of tailings is presented in this paper.

The equation for the multivariate linear regression developed to predict the moisture content of MI-mag tailings is:

$$
\begin{aligned}
\mathrm{w}= & -8.8754 \log \left(\Delta \mathrm{T}_{\text {samp }}\right)+23.4879\left(\mathrm{~T}_{\text {samp,post }}\right)^{1 / 3}+0.0290\left(\mathrm{H}_{\mathrm{pre}}\right)^{2} \\
& -51.6874 \log \left(\mathrm{H}_{\text {post }}\right)-0.0474(\Delta \mathrm{H})^{2}
\end{aligned}
$$

The predicted vs. observed plot for this regression is shown in Figure 3-8, and the variable assessment statistics for the regression are displayed in Table 3-5. Based on Equation 3-3, moisture content of the MI-mag tailings can be predicted by using pre- and post-heating sample temperature and pre- and post-heating atmospheric humidity. In this regression, post-heating sample temperature and atmospheric humidity are the most 
significant variables, while change in sample temperature is less significant but still adds value to the regression (Table 3-5). The predicted versus observed plot (Figure 3-8) shows a good relationship between modeled and measured moisture content for the MImag tailings, with a high $\mathrm{R}^{2}(0.91)$, adjusted $\mathrm{R}^{2}(0.90)$, and RMSE (3.89 percent).

Similarly, the equation for the multivariate linear regression developed to predict the moisture content of MN-mag tailings is:

$$
\begin{aligned}
\mathrm{w}= & 314.47101 /\left(\Delta \mathrm{T}_{\text {samp }}\right)+0.2091\left(\mathrm{~T}_{\text {samp,post }}\right)-2.7136\left(\mathrm{H}_{\text {pre }}\right) \\
& +0.0403\left(\mathrm{H}_{\text {post }}\right)^{2}+0.0396(\Delta \mathrm{H})^{2}
\end{aligned}
$$

The predicted vs. observed plot for this regression is shown in Figure 3-9, and the variable assessment statistics for the regression are displayed in Table 3-6. Based on Equation 3-4, moisture content of the MN-mag tailings can also be predicted by using pre- and post-heating sample temperature and pre- and post-heating atmospheric humidity. In this regression, change in sample temperature is the most significant variable, while post-heating sample temperature and pre-heating atmospheric humidity are less significant, and post-heating atmospheric humidity and change in atmospheric humidity have the least significance but still add value to the regression (Table 3-6). The predicted versus observed plot (Figure 3-9) shows a good relationship between modeled and measured moisture content for the MN-mag tailings, with a high $\mathrm{R}^{2}(0.92)$, adjusted $\mathrm{R}^{2}$ (0.91), and RMSE (5.07 percent).

There are notable differences between the regressions developed for MI-mag and MN-mag tailings. Each set of tailings requires its own equation to predict moisture content from thermal remote sensing variables. Both the magnitude of the coefficients and the variable transformations used to most effectively predict moisture content vary between the MI-mag tailings (Equation 3-3) and the MN-mag tailings (Equation 3-4). This further demonstrates the need to consider site-specific conditions, which means that all relationships need to be validated, calibrated, or re-developed for each type of tailings to be studied. 
However, there are also a number of striking similarities between these regressions. While the coefficients and the variable transformations used to predict moisture content vary between the MI-mag (Equation 3-3) and MN-mag (Equation 3-4) tailings, the actual variables used are consistent. The variables that were considered in regression development, but ultimately not utilized in the final regressions, are the same for both tailings samples; neither albedo nor atmospheric temperature added significant value to the regressions predicting moisture content. Albedo was considered in an attempt to quantify the color change associated with a change in moisture of the tailings, but the actual color change (and therefore, variation in albedo) observed during testing was less significant than anticipated, so it is not surprising that albedo was not helpful in predicting moisture content. After observing the variation in atmospheric temperature during testing (Figure 3-6 and Figure 3-7), it was expected that the variation might have an effect on the diurnal heating of the samples. Ambient temperature, however, was an input parameter when sample temperature was being measured using the FLIR thermal camera (Table 3-2). It is possible that the internal correction the thermal camera made for ambient temperature was enough to account for these variations.

Another similarity between the two regressions is that sample temperature and atmospheric humidity were the most effective variables to predict moisture content for both of the tailings. Sample temperature was expected to contribute heavily to the prediction of moisture content, because sample temperature is what is being remotely sensed and what has been shown to have a relationship with moisture content (Liu and Zhao 2006; Minacapilli et al. 2012). It is also reasonable that the atmospheric humidity would contribute to the prediction of sample moisture content. This indicates that, for future studies, when predicting moisture content from thermal remote sensing, both sample temperature and ambient humidity should be expected to contribute significantly to the sample-specific regressions being developed for tailings samples or even other soil samples. 
Table 3-5 | Variable assessment statistics for moisture content multivariate linear regression developed for MI-mag tailings.

\begin{tabular}{lccccc}
\hline \multicolumn{1}{c}{ Variable } & Estimate & Std. Error & $\mathrm{t}$ value & $\operatorname{Pr}(>|\mathrm{t}|)$ & Significance $^{*}$ \\
\hline $\log \left(\Delta \mathrm{T}_{\text {samp }}\right)$ & -8.8754 & 3.5601 & -2.493 & 0.01691 & + \\
$\left(\mathrm{T}_{\text {samp,post }}\right)^{1 / 3}$ & 23.4879 & 4.8918 & 4.801 & $2.22 \mathrm{e}-05$ & +++ \\
$\left(\mathrm{H}_{\text {pre }}\right)^{2}$ & 0.0290 & 0.0071 & 4.102 & 0.00020 & +++ \\
$\log \left(\mathrm{H}_{\text {post }}\right)$ & -51.6874 & 13.6049 & -3.799 & 0.00048 & +++ \\
$(\Delta \mathrm{H})^{2}$ & -0.0474 & 0.0122 & -3.884 & 0.00038 & +++ \\
\hline
\end{tabular}

* Significance level codes: 0-0. 1\% : '+++'; 0. 1-1\%: '‘+'; 1-5\% : '+'; 5-10\% : '’’; 10$100 \%$ : '

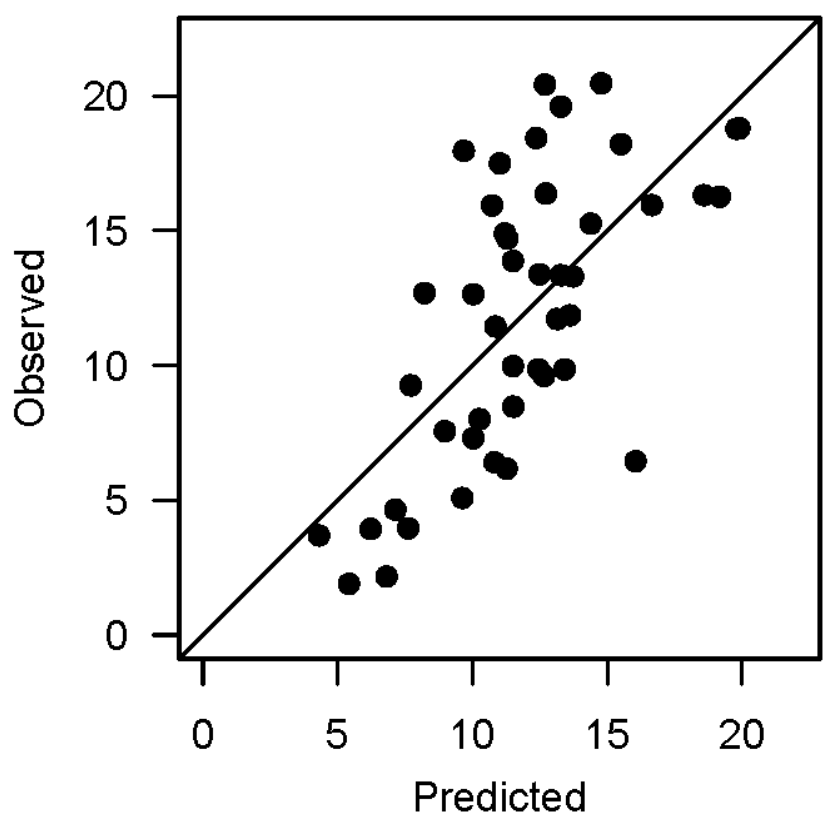

Figure 3-8 | Multivariate linear regression validation to predict moisture content for MI-mag tailings with thermal remote sensing and atmospheric variables. Points indicate daily average values for each sample, and the line shown is the 1:1 line for the data. 
Table 3-6 | Variable assessment statistics for moisture content multivariate linear regression developed for $\mathrm{MN}$-mag tailings.

\begin{tabular}{lccccc}
\hline \multicolumn{1}{c}{ Variable } & Estimate & Std. Error & $\mathrm{t}$ value & $\operatorname{Pr}(>|\mathrm{t}|)$ & Significance $^{*}$ \\
\hline $1 /\left(\Delta \mathrm{T}_{\text {samp }}\right)$ & 314.4710 & 67.2272 & 4.678 & $2.89 \mathrm{e}-05$ & +++ \\
$\mathrm{T}_{\text {samp,post }}$ & 0.2091 & 0.0595 & 3.512 & 0.00106 & ++ \\
$\mathrm{H}_{\text {pre }}$ & -2.7136 & 0.7846 & -3.459 & 0.00124 & ++ \\
$\left(\mathrm{H}_{\text {post }}\right)^{2}$ & 0.0403 & 0.0162 & 2.490 & 0.01673 & + \\
$(\Delta \mathrm{H})^{2}$ & 0.0396 & 0.0187 & 2.117 & 0.04013 & + \\
${ }^{*}$ ( & &
\end{tabular}

"Significance level codes: 0-0. 1\%: '+++'; 0. 1-1\%: ‘++'; 1-5\% : ‘+'; 5-10\%: ' ‘’; 10$100 \%$ : '

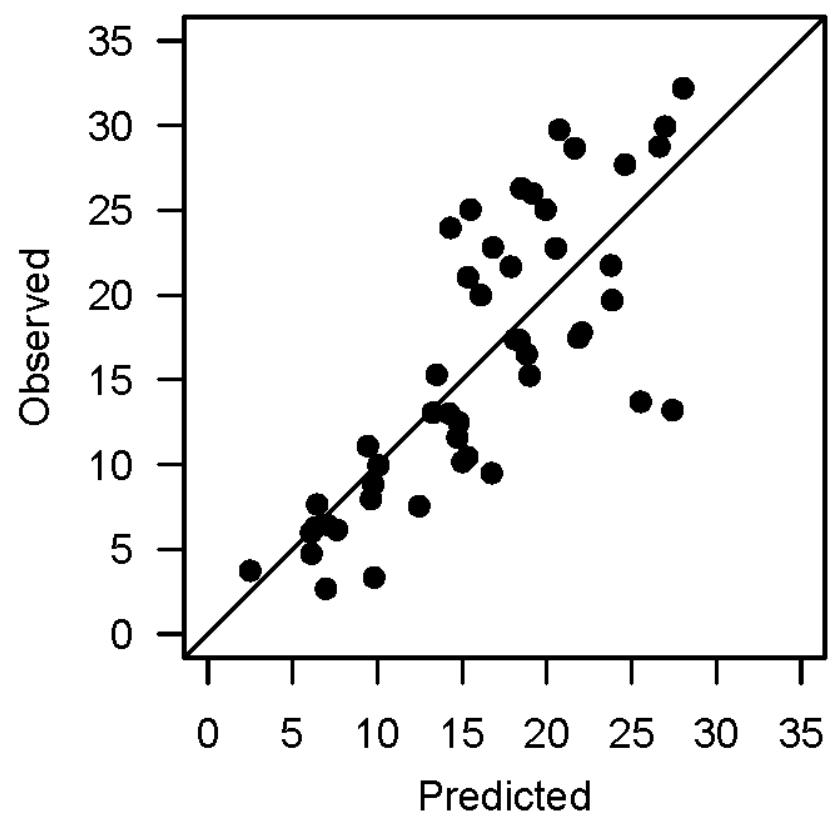

Figure 3-9 | Multivariate linear regression validation to predict moisture content for MN-mag tailings with thermal remote sensing and atmospheric variables. Points indicate daily average values for each sample, and the line shown is the 1:1 line for the data.

\subsection{Practical Applications}

For both the MI-mag (Equation 3-3) and MN-mag (Equation 3-4) tailings, this paper presents a way to predict surface gravimetric moisture content using thermal remote sensing and atmospheric variables. Using Equation 3-1 (MI-mag) and Equation 3-2 (MN- 
mag), surface strength can then be estimated for gravimetric moisture contents of 0 to 20 percent (MI-mag) and 0 to 30 percent (MN-mag). Similar relationships could be studied for other soil types, using the same methods presented in this paper which take advantage of site-specific and atmospheric conditions.

While these relationships were developed in a laboratory setting, they were developed in a way that they can be directly applied to field scale. The easiest way for these relationships to be applied to tailings impoundment monitoring is through the use of UAV-based platforms. UAV technology is rapidly developing, with UAVs becoming more affordable and sensors (including thermal sensors) becoming more lightweight and UAV-friendly. The UAV industry is booming, and the application of this research demonstrates one of many ways that UAVs can be used in the future (Liu et al. 2014). For the application of this specific research, a UAV that can carry the payload of a thermal sensor is all that is required to collect the thermal remote sensing data needed to determine surface temperature for use in Equations 3-3 and 3-4. To assess the accuracy of the application to the field before widespread application, UAV data collection must be paired with a ground truthing campaign that measures surface moisture content and strength conditions.

In addition to the thermal data that are required to apply this research, atmospheric data are also needed. Mining operations monitor atmospheric conditions such as temperature and humidity at or near their tailings impoundments, so those data are readily available to use in Equations 3-3 and 3-4. For field application, the pre-heating temperature and humidity would be collected in the early morning and could be identified as the daily low temperature, and the post-heating temperature and humidity would be collected in the afternoon and could be identified as the daily high temperature.

\subsection{Summary and Conclusions}

This study utilized laboratory testing to verify that: (1) a relationship exists between moisture content and strength for the surface of mine tailings, and (2) thermal remote sensing can be used to derive spatial variations in moisture content for the surface of two types of iron tailings. Multivariate regressions were developed to identify the critical 
remote sensing and climatic variables and evaluate their influence in the measurement of remotely measured moisture content. Based on this work, the following conclusions can be drawn:

1. A relationship does exist between moisture content and surface strength for both MI-mag and MN-mag tailings. The relationship for each tailings sample is unique, but follows similar trends, indicating that a similar relationship could be developed for other tailings samples being studied.

2. Thermal remote sensing data, specifically (pre- and post-heating) sample temperature and ambient humidity, can be used to predict moisture content for both MI-mag and MN-mag tailings. Again, the relationship for each tailings sample is unique, but a similar relationship could be developed for other tailings samples being studied.

3. Site-specific variation between tailings must be considered when studying moisture content and strength. All relationships need to be validated, calibrated, or re-developed for each type of tailings to be studied.

Future efforts will expand this study to the field scale by utilizing high-spatial resolution thermal data collected with UAVs to detect changes in moisture content for the surface of tailings impoundments.

\subsection{References}

Anderson, K., and Croft, H. (2009). "Remote sensing of soil surface properties." Progress in Physical Geography, 33(4), 457-473.

ASTM D422-63(2007), Standard Test Method for Particle-Size Analysis of Soils, ASTM International, West Conshohocken, PA, 2007, www.astm.org.

ASTM D2216-10, Standard Test Methods for Laboratory Determination of Water (Moisture) Content of Soil and Rock by Mass, ASTM International, West Conshohocken, PA, 2010, www.astm.org.

Bang, S. S., Bang, S., Frutiger, S., Nehl, L. M., and Comes, B. L. (2009) "Application of novel biological technique in dust suppression." Proc., Transportation Research Board 88th Annual Meeting.

Bindlish, R., Jackson, T. J., Gasiewski, A. J., Klein, M., and Njoku, E. G. (2006). "Soil moisture mapping and AMSR-E validation using the PSR in SMEX02." Remote Sensing of Environment, 103(2), 127-139. 
Bindlish, R., Jackson, T. J., Wood, E., Gao, H., Starks, P., Bosch, D., and Lakshmi, V. (2003). "Soil moisture estimates from TRMM Microwave Imager observations over the Southern United States." Remote Sensing of Environment, 85(4), 507515.

Buck, S., and Gerard, D. (2001). "Cleaning Up Mining Waste." Political Economy Research Center Research Study.

Cai, G., Wu, J., Xue, Y., Hu, Y., Guo, J., and Tang, J. "Soil moisture retrieval from MODIS data in northern china plain using thermal inertia model (SoA-TI)." Proc., Geoscience and Remote Sensing Symposium, 2005. IGARSS'05. Proceedings. 2005 IEEE International, IEEE, 4501-4504.

Cai, G., Xue, Y., Hu, Y., Wang, Y., Guo, J., Luo, Y., Wu, C., Zhong, S., and Qi, S. (2007). "Soil moisture retrieval from MODIS data in Northern China Plain using thermal inertia model." International journal of remote sensing, 28(16), 35673581 .

Cracknell, A., and Xue, Y. (1996). "Thermal inertia determination from space-a tutorial review." International Journal of Remote Sensing, 17(3), 431-461.

Davies, M., Martin, T., and Lighthall, P. (2000). "Tailings dam stability: essential ingredients for success." Slope Stability in Surface Mining, 365-377.

Earl, R. (1997). "Prediction of trafficability and workability from soil moisture deficit." Soil and Tillage Research, 40(3), 155-168.

Greeley, R., and Iversen, J. D. (1987). Wind as a geological process: on Earth, Mars, Venus and Titan, CUP Archive.

Jackson, T. J. (1997). "Soil moisture estimation using special satellite microwave/imager satellite data over a grassland region." Water Resources Research, 33(6), 14751484.

Jensen, J. R. (2009). Remote Sensing of the Environment: An Earth Resource Perspective Pearson Education India.

Kahle, A. B., Schieldge, J. P., and Alley, R. E. (1984). "Sensitivity of thermal inertia calculations to variations in environmental factors." Remote Sensing of Environment, 16(3), 211-232.

Liu, P., Chen, A. Y., Huang, Y.-N., Han, J.-Y., Lai, J.-S., Kang, S.-C., Wu, T.-H., Wen, M.-C., and Tsai, M.-H. (2014). "A review of rotorcraft Unmanned Aerial Vehicle (UAV) developments and applications in civil engineering." SMART STRUCTURES AND SYSTEMS, 13(6), 1065-1094.

Liu, Z., and Zhao, Y. (2006). "Research on the method for retrieving soil moisture using thermal inertia model." Science in China Series D, 49(5), 539-545.

Maltese, A., Capodici, F., Ciraolo, G., and La Loggia, G. (2013). "Mapping soil water content under sparse vegetation and changeable sky conditions: comparison of two thermal inertia approaches." Journal of Applied Remote Sensing, 7(1), 073548-073548.

Minacapilli, M., Cammalleri, C., Ciraolo, G., D'Asaro, F., Iovino, M., and Maltese, A. (2012). "Thermal inertia modeling for soil surface water content estimation: A laboratory experiment." Soil Science Society of America Journal, 76(1), 92-100. 
Müller, L., Lipiec, J., Kornecki, T. S., and Gebhardt, S. (2011). "Trafficability and workability of soils." Encyclopedia of Agrophysics, Springer, 912-924.

Murray, T., and Verhoef, A. (2007). "Moving towards a more mechanistic approach in the determination of soil heat flux from remote measurements: I. A universal approach to calculate thermal inertia." Agricultural and Forest Meteorology, 147(1), 80-87.

Murray, T., and Verhoef, A. (2007). "Moving towards a more mechanistic approach in the determination of soil heat flux from remote measurements: II. Diurnal shape of soil heat flux." Agricultural and forest meteorology, 147(1), 88-97.

Muszynski, M. R. (2000). "Void ratio distribution of normally consolidated coarsegrained magnetite tailings as a funcion of aging time." MS, Michigan Technological University.

Nickling, W. G., and Neuman, C. M. (2009). "Aeolian sediment transport." Geomorphology of Desert Environments, Springer, 517-555.

Paul, C., and Vries, J. d. (1979). "Effect of soil water status and strength on trafficability." Canadian Journal of Soil Science, 59(3), 313-324.

Petropoulos, G. P., Ireland, G., and Barrett, B. (2015). "Surface soil moisture retrievals from remote sensing: Current status, products \&amp; future trends." Physics and Chemistry of the Earth, Parts $A / B / C$.

Price, J. C. (1980). "The potential of remotely sensed thermal infrared data to infer surface soil moisture and evaporation." Water Resources Research, 16(4), 787795.

Price, J. C. (1985). "On the analysis of thermal infrared imagery: the limited utility of apparent thermal inertia." Remote sensing of Environment, 18(1), 59-73.

Price, J. C. (1998). "Evaluation and characterization of the effectiveness of dust suppressants on iron ore mine tailings." Michigan Technological University.

Putzig, N. E., and Mellon, M. T. (2007). "Apparent thermal inertia and the surface heterogeneity of Mars." Icarus, 191(1), 68-94.

Qiu, Y., and Sego, D. (2001). "Laboratory properties of mine tailings." Canadian Geotechnical Journal, 38(1), 183-190.

R Core Team. 2014. R: A language and environment for statistical computing. R Foundation for Statistical Computing, Vienna, Austria.

R Studio Team. 2015. RStudio: Integrated Development for R. RStudio, Inc., Boston, MA.

Ramakrishnan, D., Bharti, R., Singh, K., and Nithya, M. (2013). "Thermal inertia mapping and its application in mineral exploration: results from Mamandur polymetal prospect, India." Geophysical Journal International, 195(1), 357-368.

Scheidt, S., Ramsey, M., and Lancaster, N. (2010). "Determining soil moisture and sediment availability at White Sands Dune Field, New Mexico, from apparent thermal inertia data." Journal of Geophysical Research: Earth Surface (20032012), 115(F2), 1-23.

Soliman, A., Heck, R. J., Brenning, A., Brown, R., and Miller, S. (2013). "Remote sensing of soil moisture in vineyards using airborne and ground-based thermal inertia Data." Remote Sensing, 5(8), 3729-3748. 
Stovern, M., Betterton, E. A., Sáez, A. E., Villar, O. I. F., Rine, K. P., Russell, M. R., and King, M. (2014). "Modeling the emission, transport and deposition of contaminated dust from a mine tailing site." Reviews on environmental health, 29(1-2), 91-94.

USEPA (2012). "Particulate Matter (PM-10)." $<$ http://www.epa.gov/airtrends/aqtrnd95/pm10.html $>$.

USEPA (2014). "Fine Particule Matter (PM2.5) Designations." $<\mathrm{http}: / /$ www.epa.gov/pmdesignations/>.

USEPA (2014). "Mine Waste Technology." < http://www.epa.gov/nrmrl/std/mwt/>.

Vasher, D. R. (1999). "A technique for assessing fugitive particulate emission potential from an active mine tailings basin." Michigan Technological University.

Vick, S. G. (1983). Planning, Design, and Analysis of Tailings Dams, John Wiley \& Sons, Inc., New York, NY.

Wang, J., Bras, R., Sivandran, G., and Knox, R. (2010). "A simple method for the estimation of thermal inertia." Geophysical Research Letters, 37(5).

Xue, Y., and Cracknell, A. (1995). "Advanced thermal inertia modelling." Remote Sensing, 16(3), 431-446.

Zhang, R., Sun, X., and Zhu, Z. (2002). "Remote sensing information model in surface evaporation from differential thermal inertia and it's validation in Gansu Province." Science in China (Series D), 32(1), 1041-1050.

Zwissler, B., Oommen, T., and Vitton, S. (2014). "A Study of the Impacts of FreezeThaw on Cliff Recession at the Calvert Cliffs in Calvert County, Maryland." Geotech Geol Eng, 32(4), 1133-1148. 

Chapter 4 Thermal remote sensing for moisture content of mine tailings: field study ${ }^{2}$

${ }^{2}$ The material contained in this chapter is in preparation for submission to the peerreviewed literature. 


\subsection{Introduction}

Fugitive dust emissions from mine tailings impoundments are a major environmental concern for the mining industry. Mine tailings are the waste product resulting from ore beneficiation, in which the crushing and grinding operations required to separate the ore from the rock yield finely crushed rock particles - the average particle size is $20 \mu \mathrm{m}$, with a significant portion between 1 and $10 \mu \mathrm{m}$. The tailings are often deposited in slurry form into permanent tailings impoundments, which are among the largest earthen structures in the world (Vick 1983). Over 3500 active tailings impoundments exist worldwide (Davies et al. 2000).

Due to the small size of tailings particles and the large volume of tailings stored in impoundments, air pollution from blowing dust is a significant environmental concern (Buck and Gerard 2001; Stovern et al. 2014), and is regulated by the USEPA under the Clean Air Act as airborne particulate matter based on particle size and particle concentration (USEPA 2012; USEPA 2014). Blowing dust due to wind erosion of mine tailings can negatively impact human activity via poor visibility for drivers and respiratory health problems (Bang et al. 2009).

To control fugitive dust emissions, it is crucial to understand how the tailings are affected by wind erosion and how to detect when they may be susceptible to dusting. Typically, the lower the moisture content of the tailings is, the higher the dust susceptibility (Greeley and Iversen 1987; Nickling and McKenna Neuman 2009). However, even for well-studied tailings impoundments, it is difficult to make static and general characterizations about the tailings properties without considering spatial and temporal variations in the dynamic impoundment. Understanding how the moisture content changes spatially and temporally is complex, so monitoring the tailings to determine if and when certain regions of the impoundment may be susceptible to dusting is important. The traditional approach for monitoring dust emissions from tailings is reactive in nature rather than proactive, with a focus on daily visual inspections of the impoundments and collecting dust samples from gauges. This approach provides limited 
spatial coverage of the tailings, and is also costly, labor intensive, and affected by trafficability. Because of these limitations, the problem may not be detected before the dusting event occurs.

In addition to the actual hazard of blowing dust, trafficability can also be a concern for tailings impoundment managers, because high surface moisture can result in mine equipment losing bearing capacity while inspecting regions of the impoundment and/or applying dust control measures. Trafficability is also related to the surface moisture content of the tailings, and can be defined as the ability of the surface tailings to support the traffic of heavy equipment (Müller et al. 2011). Trafficabilty concerns include the ability of tailings to provide adequate traction for vehicles and the ability to resist excessive compaction and structural damage (Earl 1997; Paul and Vries 1979). While the hazard of fugitive dust emissions is caused by areas of tailings with low moisture content, accessing these regions for mitigation activities can be limited by regions of high moisture and low trafficability. Being able to identify regions of low trafficability is critical during planning and execution of dust mitigation operations.

The ever-changing surface conditions of any active tailings impoundment make it difficult to try to characterize the surface conditions of the tailings. For dust susceptibility and trafficability monitoring, remote sensing may be an effective method for monitoring the surface of tailings impoundments, because it provides a synoptic view of surficial processes with high spatial and temporal resolutions, making it useful for monitoring and detecting change. To utilize the high spatial and temporal resolution that remote sensing enables for monitoring the impoundment surface moisture content and strength, it is critical to derive the moisture content in the top layer of the tailings using remote sensing. Thermal remote sensing has been proven to be a useful tool to (in)directly detect soil moisture (Liu and Zhao 2006; Minacapilli et al. 2012).

Therefore, Zwissler et al. (in review) carried out a laboratory study, described in more detail below, to develop a model to predict surface moisture content and strength for magnetite tailings using thermal remote sensing. This model was developed utilizing actual tailings samples prepared to moisture content and density conditions representative 
of field conditions, and included variables to account for atmospheric conditions, with the goal of being able to be used in the field. However, the applicability of the laboratory model to a field setting needs to be validated before it can be used by tailings impoundment managers as a dust susceptibility monitoring tool. Thus, the objective of this paper is to assess the validity of applying the laboratory-based model developed by Zwissler et al. (in review) to field scale. This was achieved by using field-based thermal remote sensing coupled with moisture content and strength ground truthing to apply and assess the model.

\subsection{Description of Laboratory Model}

A summary of the laboratory model developed by Zwissler et al. (in review) is described in this section, so that an understanding of the similarities between the laboratory and field conditions can be assessed. More details about the geotechnical characterization of samples, laboratory experiments, and model development can be found in Chapter 3 and Zwissler et al. (in review).

Laboratory testing was conducted using magnetite tailings samples from an impoundment in Michigan (called MI-mag tailings). The MI-mag tailings were 95 percent silt-sized particles and 5 percent clay-sized particles. All laboratory samples were prepared at void ratios between 0.8 and 1.0 in $76 \mathrm{~mm} \times 76 \mathrm{~mm} \times 76 \mathrm{~mm}$ polycarbonate soil boxes and fully saturated using a Mariotte tube constant head device, to mimic field conditions (Price 1998).

Samples were exposed to an artificial diurnal heating cycle daily (6 hours daily under two 500-Watt halogen lamps and two full spectrum lights), and were monitored and tested until the samples were dry. This approach provided a range of moisture/strength conditions per sample over the duration of testing. A suite of measurements were taken twice daily for samples - once before heating, called "pre-heating", and once after heating, called "post-heating. These measurements included: gravimetric moisture content by monitoring sample mass loss on a balance; relative strength via a needle 
penetrometer; atmospheric temperature $(\mathrm{K})$ and relative humidity (percent) via a thermohygrometer; and sample temperature $(\mathrm{K})$ using a FLIR ThermaCAM SC640 thermal camera.

Testing of the MI-mag tailings occurred during the summer of 2014 and yielded 45 daily average data points. Testing was conducted during the summer in a laboratory lacking air conditioning or temperature/humidity control, so atmospheric variation captured building temperature/humidity fluctuations as well as the effects of the diurnal heating cycle. During the laboratory testing of the MI-mag, the atmospheric temperature in the laboratory ranged from $294.6 \mathrm{~K}$ to $325.4 \mathrm{~K}$, with $\Delta \mathrm{T}$ ranging from $18.3 \mathrm{~K}$ to 26.2 $\mathrm{K}$. The relative atmospheric humidity in the laboratory ranged from 13 percent to 64 percent, with $\Delta \mathrm{H}$ ranging from 16 percent to 42 percent.

After all data were collected, the relationships between the measured variables for each set of tailings were explored using regression analysis. For the MI-mag tailings, the final regressions used sample temperature and atmospheric humidity to predict moisture content of the tailings, and did not use atmospheric temperature. The final model from Zwissler et al. (in review) for predicting moisture content of the MI-mag tailings $\left(\mathrm{R}^{2}=0.91\right.$, adjusted $\mathrm{R}^{2}=0.90, \mathrm{RMSE}=3.89$ percent $)$, valid for moisture contents up to 20 percent, is:

$$
\begin{aligned}
\mathrm{W}= & -8.8754 \log \left(\Delta \mathrm{T}_{\text {samp }}\right)+23.4879\left(\mathrm{~T}_{\text {samp,post }}\right)^{1 / 3}+0.0290\left(\mathrm{H}_{\text {pre }}\right)^{2} \\
& -51.6874 \log \left(\mathrm{H}_{\text {post }}\right)-0.0474(\Delta \mathrm{H})^{2}
\end{aligned}
$$

where $\Delta \mathrm{T}_{\text {samp }}$ is the change in sample temperature (K) due to diurnal heating, $\mathrm{T}_{\text {samp,post }}$ is the sample temperature $(\mathrm{K})$ post-heating, $\mathrm{H}_{\text {pre }}$ is the relative atmospheric humidity (percent) pre-heating, $\mathrm{H}_{\text {post }}$ is the relative atmospheric humidity (percent) post-heating, and $\Delta \mathrm{H}$ is the change in relative atmospheric humidity (percent) due to diurnal heating. 


\subsection{Application of Laboratory Model to Field Scale}

Field validation of the laboratory models was conducted at the MI-mag tailings impoundment on June 21, 2016. During field testing at the MI-mag tailings impoundment, fifteen sites along the eastern side of the impoundment that were visually identified to have varying moisture contents were identified, marked using spray paint and flagging, and tested. Details of the testing are described below.

Upon arrival to the tailings impoundment, each site was marked with spray paint using a template containing an inner $0.3 \mathrm{~m}$ x $0.3 \mathrm{~m}$ square and an outer $0.45 \mathrm{~m} \times 0.45 \mathrm{~m}$ square. The inner square remained undisturbed, and was the target used for thermal imagery. The tailings in the outer square were used for moisture content sampling and strength testing, and due to their close proximity to the inner square, were assumed to represent the tailings captured with thermal imagery. This marking and testing scheme is outlined in Figure 4-1. As soon as all sites were marked, morning "pre-heating" testing was conducted from 8:30 to 9:30 AM, which was as soon as possible based on site access limit. Even though the sun was up during this period, not much notable diurnal heating occurred during this time. Atmospheric temperature during this one-hour morning testing window ranged from $287.8 \mathrm{~K}$ to $294.8 \mathrm{~K}($ mean $=290.5$, standard deviation $=1.9)$, and relative humidity ranged from 51.0 percent to 61.2 percent $($ mean $=54.8$, standard deviation $=3.1$ ). Due to equipment issues, the afternoon "post-heating" testing was conducted from 3:30 to 4:30 PM, yielding a 7 hour diurnal heating cycle, which is a slight but unavoidable deviation from the laboratory 6 hour cycle. The atmospheric temperature during this one-hour afternoon testing window ranged from $295.7 \mathrm{~K}$ to 297.2 $\mathrm{K}($ mean $=296.4$, standard deviation $=1.1)$, and relative humidity ranged from 30.3 percent to 32.7 percent $($ mean $=31.5$, standard deviation $=1.7)$.

The measurements made during the pre-heating and post-heating testing are outlined in Table 4-1, and the details of these tests follow. Location details were measured using a handheld GPS unit, and was used for spatial visualization of the sampling sites. Atmospheric temperature $(\mathrm{K})$ and relative humidity (\%) were measured using a 
thermohygrometer at each location during both pre- and post-diurnal heating. Tailings samples were collected using either a shovel or spoon and placed into a bucket or bag for transport back to the laboratory. Care was taken when collecting tailings to sample only the surface material (top $5 \mathrm{~cm}$ ). Gravimetric moisture content (\% by mass) was determined by for each sample collected following ASTM D2216-10. The daily average moisture content for each site was used in data analysis. Relative soil strength was quantified using a handheld vane shear device. The vane shear measured degrees of rotation, which was converted to shear strength using ASTM 4648-16. All vane shear measurements were made at the tailings surface. At least 5 measurements were taken per sample, and the average (daily) value was used to represent tailings strength. Change in surface temperature was measured using a FLIR ThermaCAM SC640 thermal camera. For the thermal camera to measure surface temperature rather than radiant energy, FLIR object parameters were measured and used each time an image was taken as described in Table 4-2. The surface temperature of each sample, which was used to calculate the change in surface temperature, was determined using FLIR ThermaCAM Researcher software. A sample of a pre-heating and post-heating thermal image is presented in Figure 4-2. 


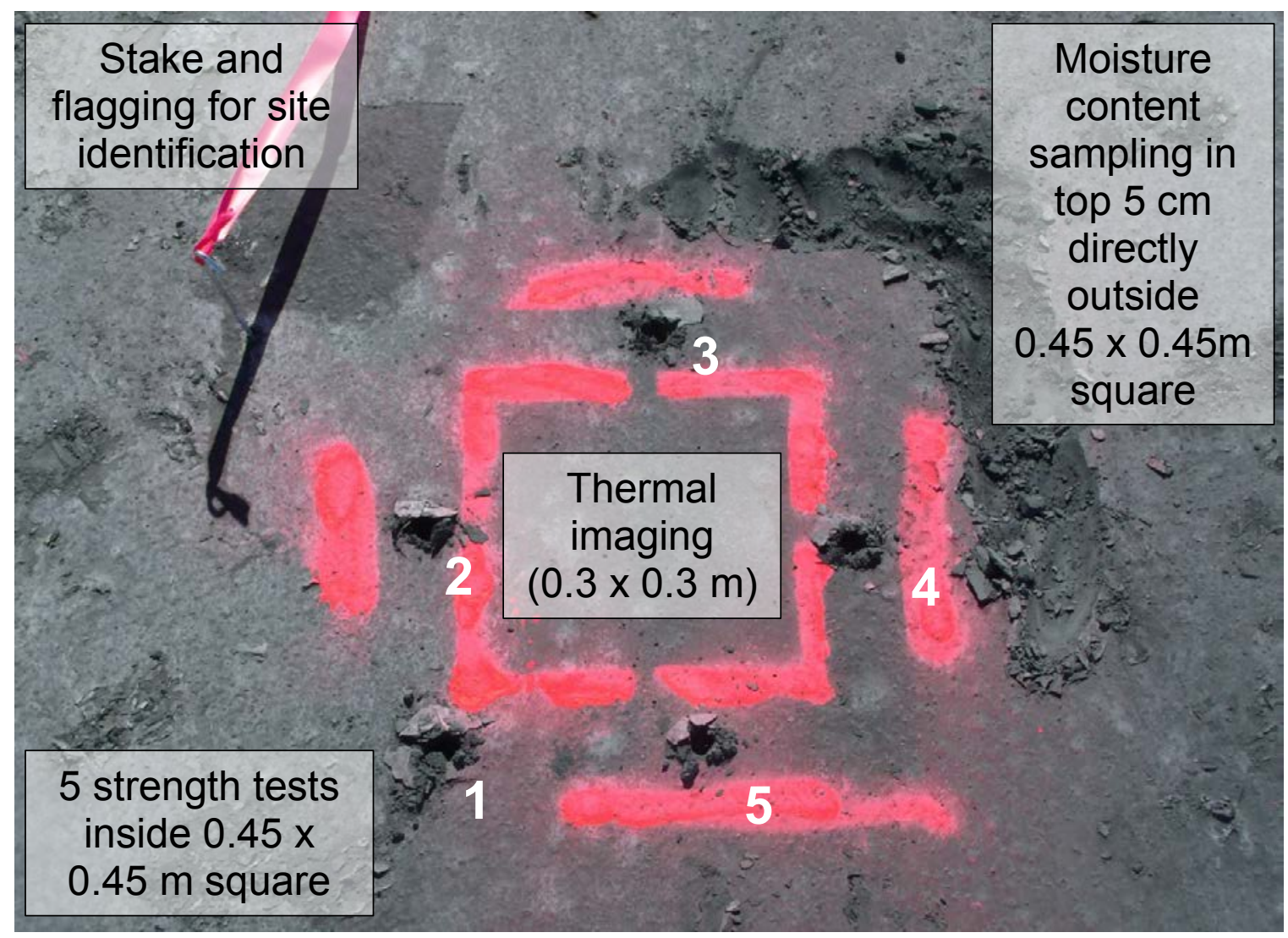

Figure 4-1 | Site marking and testing scheme used for fifteen study sites at MI-mag tailings impoundment in June 2016. 
Table 4-1 | Details of field testing in June 2016 for fifteen sites on the eastern side of the MI-Mag tailings impoundment, where measurements were taken pre- and postheating due to a 7 hour diurnal heating cycle.

\begin{tabular}{llll}
\hline \multicolumn{1}{c}{ Data Collected } & Equipment Used & \multicolumn{1}{c}{ Purpose } & Tests per Site \\
\hline Location details & GPS & $\begin{array}{l}\text { To be able to relate tailings data } \\
\text { to satellite/UAV data }\end{array}$ & 1 measurement \\
\hline $\begin{array}{l}\text { Atm. temp. \& } \\
\text { humidity }\end{array}$ & $\begin{array}{l}\text { Thermo- } \\
\text { hygrometer }\end{array}$ & $\begin{array}{l}\text { To quantify atmospheric } \\
\text { conditions across impoundment }\end{array}$ & $\begin{array}{l}1 \text { measurement, } \\
\text { pre/post heating }\end{array}$ \\
\hline Tailings samples & Spoon \& bag & $\begin{array}{l}\text { For moisture content } \\
\text { characterization in lab }\end{array}$ & $\begin{array}{l}1 \text { sample, } \\
\text { pre/post heating }\end{array}$ \\
\hline Tailings strength & Vane shear & $\begin{array}{l}\text { To quantify surface strength of } \\
\text { tailings }\end{array}$ & $\begin{array}{l}5 \text { per location, } \\
\text { pre/post heating }\end{array}$ \\
\hline $\begin{array}{l}\text { Thermal images } \\
\text { of tailings }\end{array}$ & $\begin{array}{l}\text { FLIR SC 640 } \\
\text { camera }\end{array}$ & $\begin{array}{l}\text { To quantify surface } \\
\text { temperature variation \& to } \\
\text { calculate ATI }\end{array}$ & $\begin{array}{l}5 \text { images, } \\
\text { pre/post heating }\end{array}$ \\
\hline
\end{tabular}

Table 4-2 | Description of each FLIR object parameter and how the parameter was quantified.

\begin{tabular}{|c|c|}
\hline Object Parameter & How to Quantify Each Parameter \\
\hline Emissivity & Determined by Zwissler et al. (in review) to be 0.9 \\
\hline Reflected apparent temperature $(\mathrm{K})$ & Same value as atmospheric temperature \\
\hline Object distance $(\mathrm{m})$ & $\begin{array}{l}1.2 \text { meters (dist. between tailings surface and camera } \\
\text { lens) }\end{array}$ \\
\hline Atmospheric temperature $(\mathrm{K})$ & $\begin{array}{l}\text { Measured with thermohygrometer at each sample } \\
\text { location }\end{array}$ \\
\hline Relative humidity (\%) & $\begin{array}{l}\text { Measured with thermohygrometer at each sample } \\
\text { location }\end{array}$ \\
\hline $\begin{array}{l}\text { External optics temperature }(\mathrm{K}) \text { and } \\
\text { transmission }\end{array}$ & $\begin{array}{l}1.0 \text { (value to be used when no external optics for } \\
\text { camera) }\end{array}$ \\
\hline
\end{tabular}

Figure 4-2 | Thermal infrared imagery of MI-mag tailings impoundment collected (left) pre-heating, and (right) post-heating, in June 2016. Dark colors represent lower relative temperatures, and bright colors represent higher relative temperatures. Average temperature was determined for the tailings inside the $0.3 \mathrm{~m} \mathrm{x} 0.3 \mathrm{~m}$ spray paint target. 


\subsection{Results and Discussion of Field Testing}

Field data, including moisture content, relative strength, atmospheric temperature, relative atmospheric humidity, and surface temperature were measured at fifteen study sites at the MI-mag tailings impoundment, both before and after a diurnal heating cycle. The relationships between these variables were explored, and the field data collected was used to apply the laboratory-based model (Equation 4-1). The results are presented and discussed below.

First, the relationship between moisture content and change in surface temperature was assessed. The magnitude of soil temperature change, with respect to atmospheric temperature change, is a function of soil moisture, so a trend between these variables should exist. A plot of the relationship between moisture content and change in surface temperature is shown in Figure 4-3. This relationship, which has a coefficient of correlation (r) of 0.89 , demonstrates that thermal remote sensing can be used to detect surface moisture content, especially at lower moisture contents, with surprisingly high predictive capability. However, the relationship shown in Figure 4-3 should be viewed with some caution, because laboratory testing (Figure 4-4) showed that the relationship between moisture content and change in surface temperature alone is not a reliable way to predict moisture content $(\mathrm{r}=0.50)$. While the overall trend between moisture content and change in surface temperature was the same for both laboratory and field samples, with an increase in moisture content leading to a decrease in $\Delta \mathrm{T}_{\mathrm{samp}}$, there is notably more scatter in the laboratory data. As discussed more below, there were differences between the laboratory and field testing conditions, including light/heat source, wind, and differences in atmospheric conditions (over an entire summer for the laboratory data and over a single day for the field data), which likely account for the lack of strength in the laboratory-based relationship.

Next, the relationship between moisture content and relative surface strength was analyzed, and compared to the laboratory relationship studied by Zwissler et al. (in review). A plot of the trend between moisture content and relative strength for both the 
laboratory and field MI-mag data is shown in Figure 4-5. It should be noted that relative strength from a needle penetrometer, used for laboratory testing, is the inverse of penetration depth, while the relative strength from a vane shear device, used for field testing, is proportional to degrees of rotation. It is shown in Figure 4-5 that, for higher moisture contents (those greater than approximately $5 \%$ ), trends in relative strength measured for both laboratory samples (using a needle penetrometer) and field samples (using a vane shear device) agree. However, the field data presented in Figure 4-5 also highlights a limitation of using the vane shear device for low moisture contents in tailings samples (those less than approximately 5\%). Due to the composition of the tailings, namely the small size of the tailings particles/voids and the resulting high suction in the soil matrix, tailings dry into an incredibly hard mass. For strength testing using a vane shear device, the vane (height $=28.60 \mathrm{~mm}$, diameter $=19.05 \mathrm{~mm}$ ) has to be completely inserted into the soil before rotation for results to be consistent and accurate. At these low moisture contents, to insert the vane completely into the tailings, a good amount of disturbance occurred to the hardened tailings, including inducing radial cracking from the vane outward. When the vane was inserted and then rotated, this disturbed and cracked soil provided less resistance to failure and therefore required lower degrees of rotation before failing.

It should be noted that, in addition to a vane shear, a handheld cone penetrometer (cone height $=36.85 \mathrm{~mm}$, diameter $=20.65 \mathrm{~mm}$ ) was brought to the MI-mag tailings impoundment with the intention of using it for additional relative strength testing. However, for the majority of the study sites selected, the tailings were too strong for the cone to be manually penetrated. It is suspected, though not confirmed, that a smaller cone would have led to more successful testing, but caution would need to be used so as to not bend the rod while driving the cone. Future in-situ strength testing of tailings, especially when low moisture content samples are desired, should account for the downfalls of vane shear and cone penetrometer testing and plan accordingly.

Lastly, the field data was used to apply the laboratory-based model (Equation 4-1). Because the model is valid for moisture contents below $20 \%$, the three study sites with 
moisture contents above $20 \%$ were excluded, leaving 12 data points. The application of the MI-mag laboratory model to the field data collected on June 21 at the MI-mag tailings impoundment is shown in Figure 4-6. As seen in the observed versus predicted plot, the model significantly over-predicts moisture content. However, it is worth noting the differences between the laboratory testing and field testing conditions, which could play a part in the variation between the model performance for the laboratory and field data.

The first variation to note between the laboratory testing used to develop the model and the field testing used to assess the model is atmospheric temperature. While the laboratory model was developed considering the effects of atmospheric temperature, atmospheric temperature was ultimately found to be an insignificant variable and not included in the final model. It is possible that accounting for atmospheric temperature is more important for field testing than it was for laboratory testing. The atmospheric temperatures seen during field testing were lower than those observed during laboratory testing, though they fell close to the range observed in laboratory testing. The effects of these differences on model performance are note expected to be large, but are untested.

The second variation to note is diurnal heating source and time. In laboratory testing, diurnal heating was achieved using two full-spectrum and two halogen lights for six hours, while in field testing, diurnal heating was driven by seven hours of exposure from the sun. The heating time difference was likely negligible, because the overall change in surface temperatures observed in the field from diurnal heating fall within the range of values seen in laboratory testing. However, it is possible that other, unquantified differences between using lamps and the sun as a heating source affected the tailings in an unexpected way.

The geotechnical properties of the tailings, namely density and grain size, could be other noteworthy variations between laboratory and field conditions. The laboratory samples were all prepared to a uniform density from the same batch of tailings with a quantified grain size distribution. Due to time limitations, the density of the tailings at the fifteen study sites was not measured, and was assumed to fall within the range documented by Price (1998). Also due to time limitations, the grain size of the fifteen 
study sites was qualitatively assesd and deemed to be primarily fine-grained material, but no formal grain-size testing was performed. It is possible that variations in grain size and density affect the diurnal heating of the tailings.

The last, and most significant, variations to note are the other environmental conditions that were not present in laboratory testing and not quantified by the laboratory model. These environmental conditions include, but are not limited to, wind and the presence of clouds. There was never any source of wind present in the laboratory testing, nor was the potential effect of wind accounted for in the laboratory-based model.

However, there was significant wind during the field testing. Sustained winds estimated to be at least 30 to $50 \mathrm{~km} / \mathrm{hr}$, with gusts even higher, were present at the tailings impoundment on the day preceding testing and also on the day of field testing. The authors suspect that the wind could have affected tailings density, diurnal heating, and other factors that would impact the relationship between moisture content and surface temperature. Another environmental factor unaccounted for in the laboratory was the presence of clouds. The day of field testing was largely cloud-free, but clouds were present during the last one to two hours of heating and testing, which could have also had effects that were unaccounted for.

Still, returning to Figure 4-3, this field data does suggest that there should be a way to use thermal remote sensing to predict moisture content for tailings in the field. Likely, a model would need to be developed based on field-based data, rather than using a model developed with laboratory data. As desirable as laboratory testing can be, due primarily to convenience for repeated data collection and relative control over test conditions, it makes sense that a model aimed at predicting field conditions would be best served being developed using field data. 


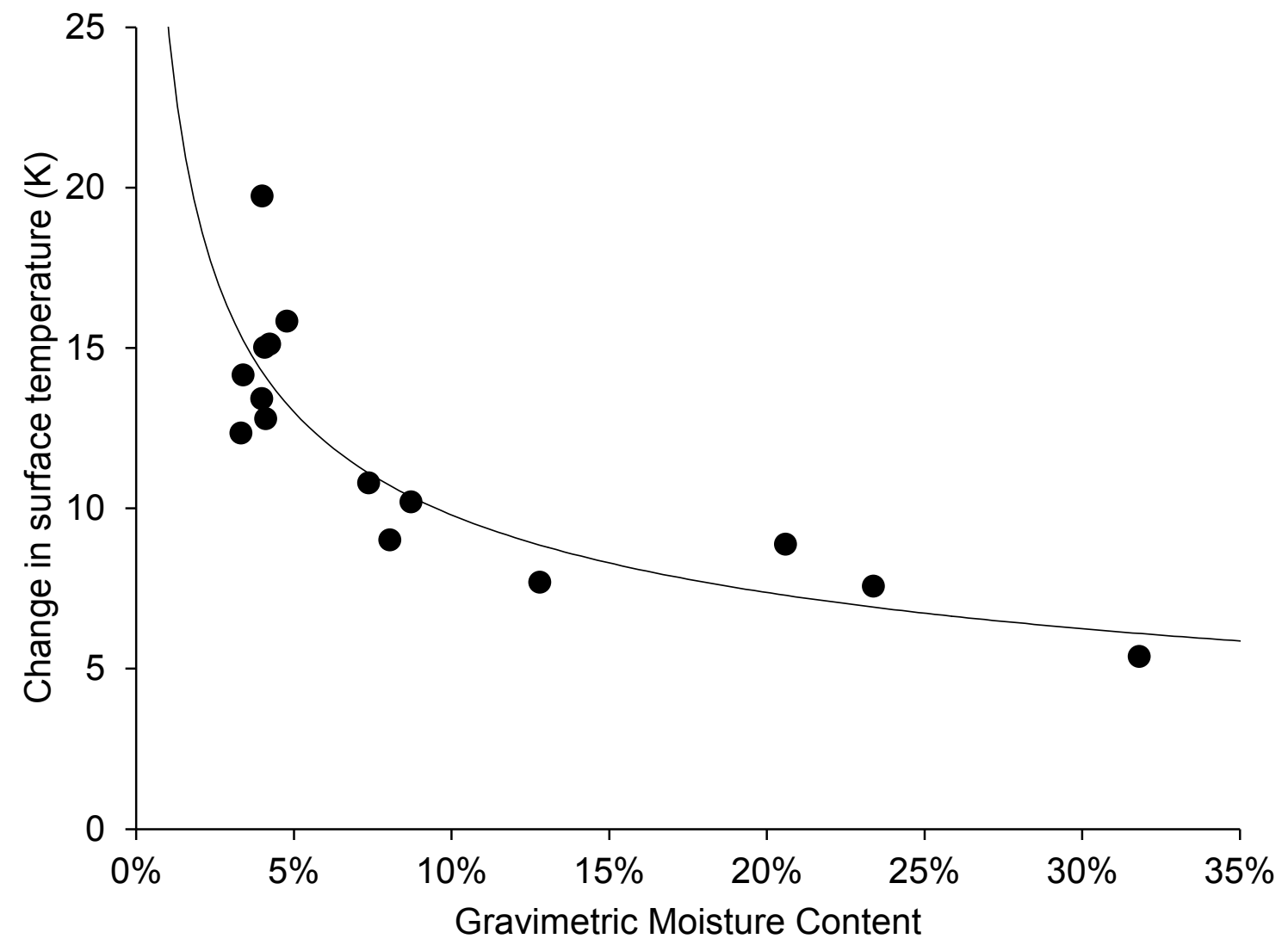

Figure 4-3 | Relationship between moisture content and surface temperature for fifteen study sites at MI-mag tailings impoundment in June $2016\left(\mathrm{R}^{2}=0.80\right)$. 


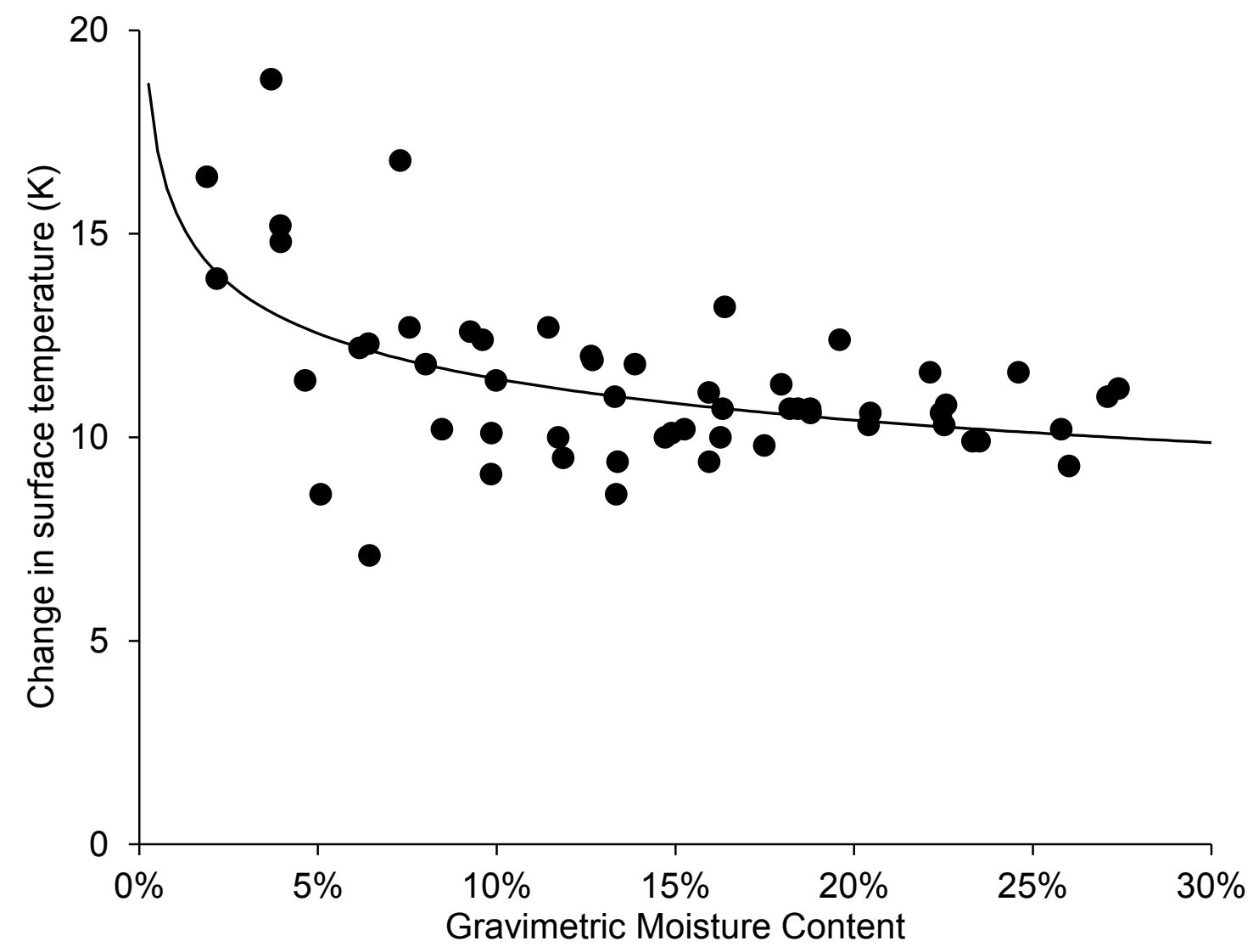

Figure 4-4 | Relationship between moisture content and surface temperature for laboratory MI-mag tailings samples tested in summer $2014\left(\mathrm{R}^{2}=0.25\right)$. Each point represents the daily average value for a single sample. 


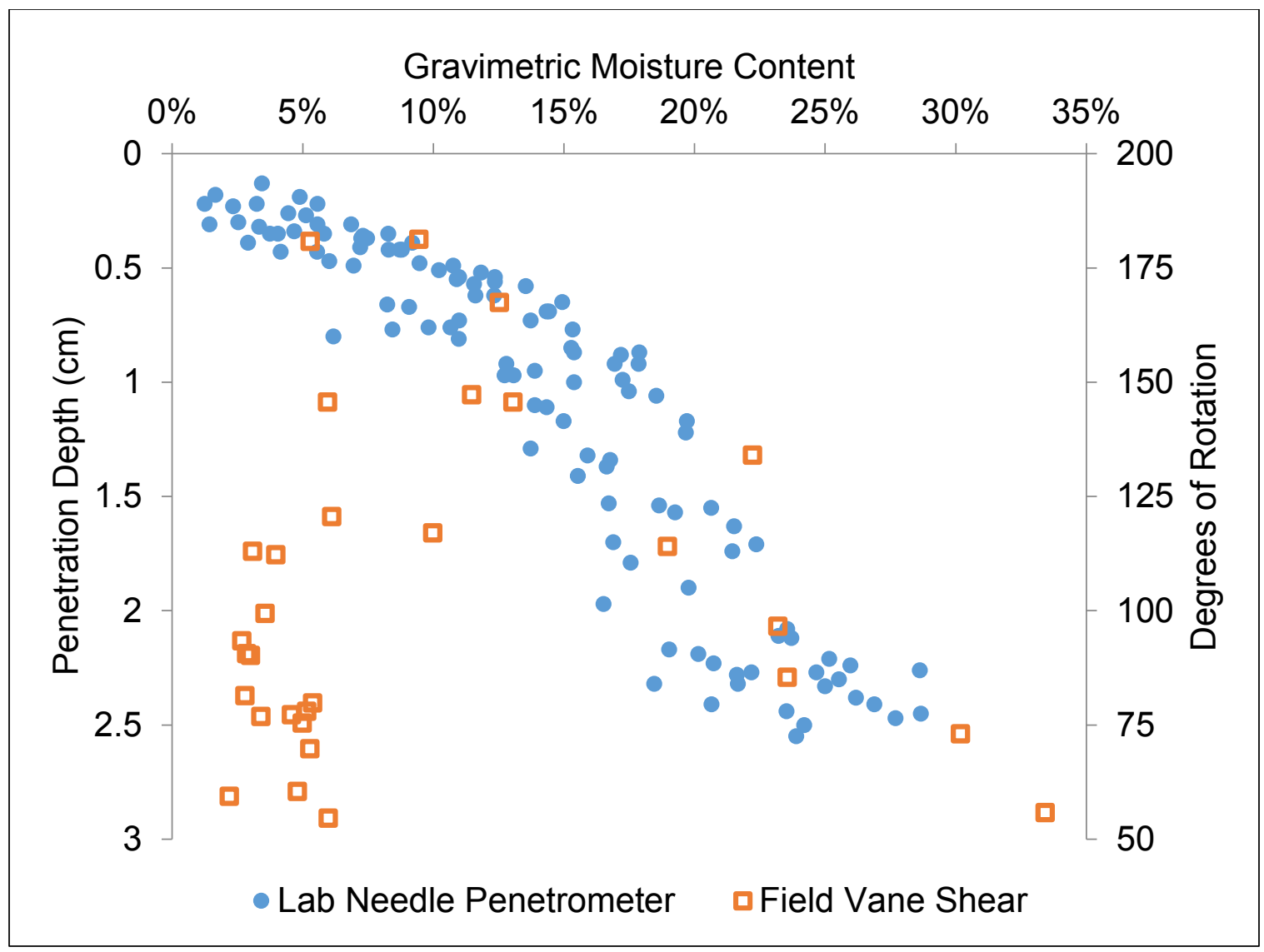

Figure 4-5 | Relationship between moisture content and relative surface strength for MI-mag tailings. Laboratory strength data $(n=116)$ were measured using a needle penetrometer, where penetration depth represents the inverse of relative strength. Field strength data $(n=30)$ were measured using a vane shear device, where degrees of rotation represents relative strength. Each point represents a single measurement. 


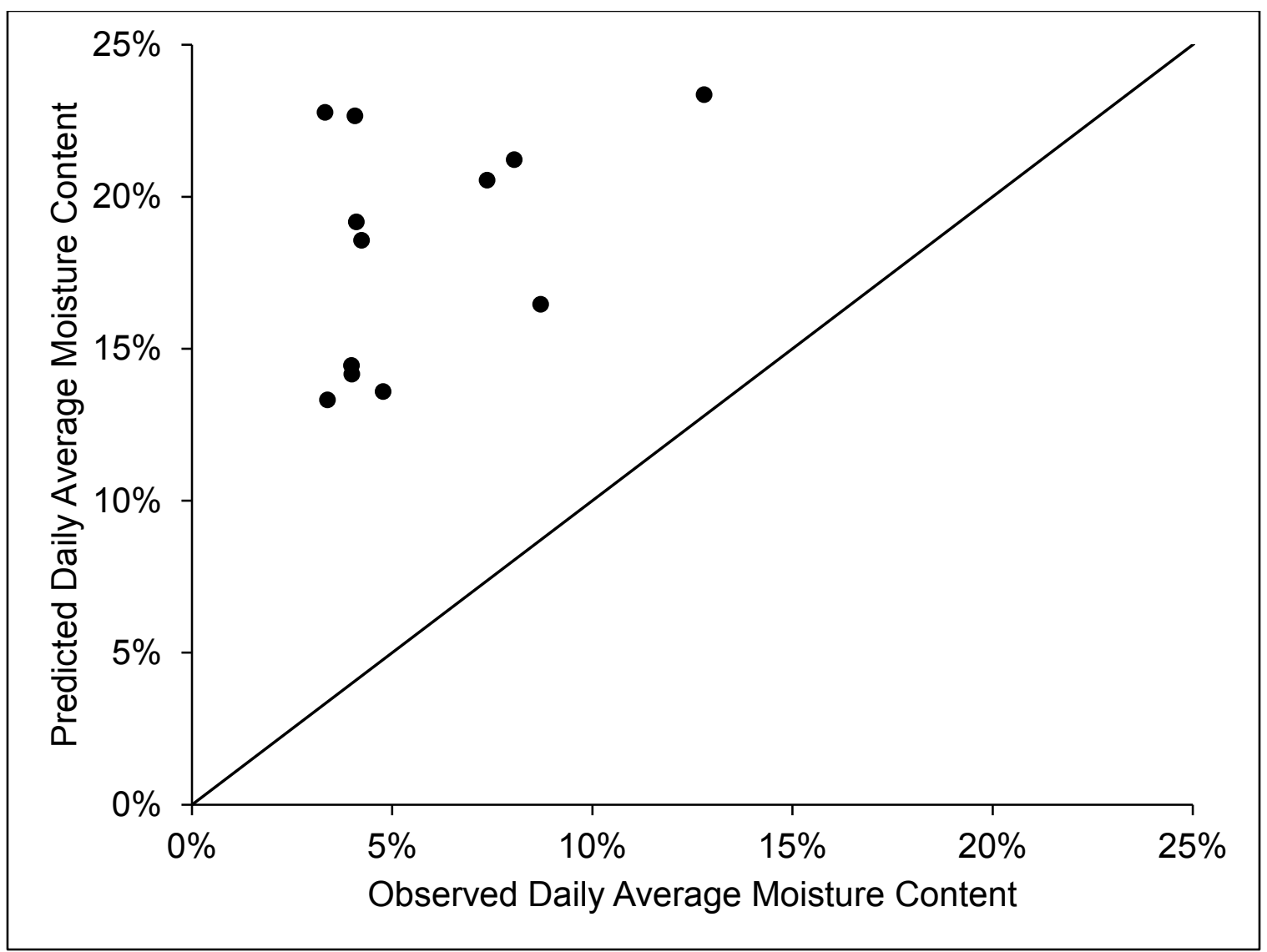

Figure 4-6 | Model validation for using laboratory-developed model for field data $(n=12)$ from MI-mag tailings collected in June 2016. Each point represents data from a single site.

\subsection{Future Work Required for Effective Field Application}

The laboratory model developed by Zwissler et al. (in review) was not proven to be directly applicable to predict moisture content for field data, based on the field data collected for the MI-mag tailings impoundment. However, the data presented in Figure 4-3 and Figure 4-5 shows promise that there should be a way to use thermal remote sensing to predict moisture content and surface strength for tailings in the field. Likely, a model would need to be developed based on field-based data, rather than using a model developed with laboratory data. Beyond troubleshooting the issues with the laboratory 
based model, there are other challenges that need to be overcome. The largest challenges include collecting and processing the thermal imagery.

The thermal images used in this study were discrete images collected with a handheld thermal camera, rather than using spatially-continuous imagery over the entire tailings impoundment. The thermal images used did satisfy the objective of assessing whether the laboratory relationships between thermal remote sensing and moisture content/strength can be applied to field scales. However, for full-scale industrial application, it would be more useful to collect spatially-continuous imagery over an entire impoundment so that maps can be generated to identify spatial and temporal changes in moisture content/strength for tailings impoundments, which could prove to be useful dust management tools to tailings impoundment managers. Developing methods to produce such maps was outside of the scope of this study, but some proof-of concept work on such applications was conducted.

Remote sensing data with continuous spatial coverage is typically collected using satellite, airborne, UAV, and ground-based imagery. Due to concerns about implementation cost, spatial coverage, spatial resolution, and temporal resolution of the imagery, UAV remote sensing look to be the most promising techniques for use at tailings impoundments. Benefits of UAV-based remote sensing include that imagery can be flown on-demand with any sensor that the UAV can carry, meaning that pre- and postheating thermal imagery could be collected on the same day. Additionally, the low flying height of UAVs means that clouds do not affect data acquisition like the do for satellite data, and the spatial resolution of all imagery collected is high (often $\mathrm{cm}$ scale). However, a major drawback of UAV-based remote sensing is the intensive data processing that is required, especially if the UAV flying height varies and if the UAV imagery is collected without GPS tracking. This processing is complex because UAV-based remote sensing yields overlapping, discrete images that need to be scaled, mosaicked, and georeferenced prior to interpretation. 
To assess the feasibility of using a UAV to collect thermal images, a FLIR Tau 2 thermal imaging camera attached to a DJI Phantom quadcopter UAV was flown on May 26, 2015 and used to collect thermal images of the surface of the MN-mag tailings basin. The Tau 2 thermal camera collects a single band of data $(7.5-13.5 \mu \mathrm{m})$ that could easily be calibrated with surface conditions and used to map spatial and temporal changes in thermal properties, moisture content, and strength for the tailings.

While the UAV-based thermal imagery was not processed to calculate surface temperature, it was qualitatively assessed, and sample images can be seen in Figure 4-7. A remarkable level of detail was distinguishable, and variations in relative temperature were observed, so the proof-of-concept UAV flight indicates that this technology could be used to map spatial and temporal changes of the surface of tailings impoundment with a high level of detail. This UAV imagery, when processed and paired with atmospheric and ground data, shows promise that it would enable the mapping of spatial and temporal changes in moisture content and strength across entire tailings impoundment(s).

The reason that this proof-of-concept UAV thermal imagery was not processed to calculate surface temperature is because of issues with scaling and stitching together the discrete thermal images to create a mosaic and georeference. The pattern recognition algorithms typically used by software designed to create image mosaics from traditional RBG visible imagery was not found to be effective at matching these thermal images. Due to a low flying height, ground control targets were not visible in every thermal image, and without automated image matching to assist in the image stitching, the task of creating a single scaled and georeferenced mosaic was found to be virtually impossible.

In order for these UAV-based thermal remote sensing methods to be applied, the technology needs to be developed to process this thermal imagery effectively, to yield a georeferenced thermal mosaic that can be compared to ground truth data. When this exists, UAV-based thermal imagery can be collected and applied to much larger surface areas very easily. 

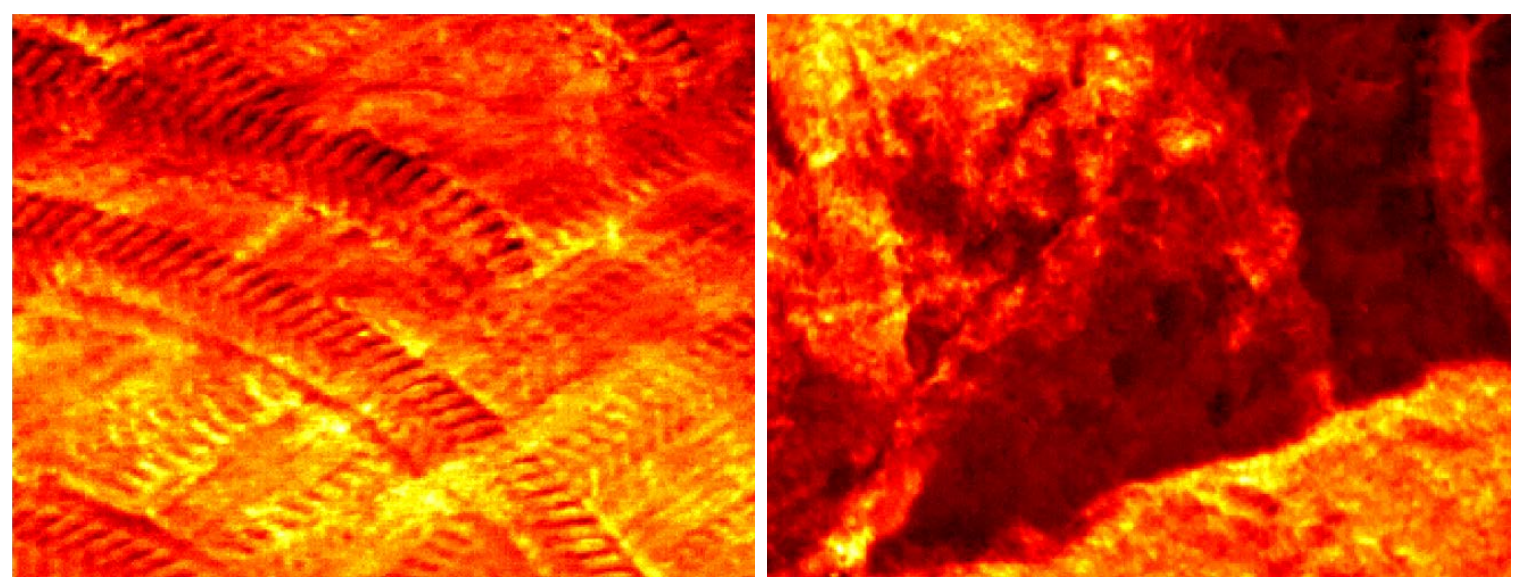

Figure 4-7 | Thermal infrared imagery of MN-mag tailings impoundment collected with UAV in May 2015. Dark colors represent lower relative temperatures, and bright colors represent higher relative temperatures. Detail including tire tracks can be distinguished in this imagery (left).

\subsection{Conclusions}

This study was aimed at assessing the application of the laboratory-based model developed by Zwissler et al. (in review) to field scale. This was achieved by using fieldbased thermal remote sensing coupled with moisture content and strength ground truthing and monitoring of atmospheric conditions at the MI-mag tailings impoundment to apply and assess the model. The results of this field study are that:

- The data collected shows that thermal remote sensing can be used to detect surface moisture content, especially at lower moisture contents, with high predictive capability $(\mathrm{r}=0.89)$.

- For moisture contents greater than 5 percent, a relationship exists between moisture content and relative strength, which agrees with laboratory data; it was discovered that vane shear testing does not yield reliable or reasonable strength values for low moisture content tailings.

- The laboratory-based model significantly over-predicts field moisture content, though the differences between the laboratory testing and field testing conditions (including atmospheric temperature, diurnal heating source/time, tailings density, 
tailings grain size, the presence of clouds, and wind) could play a part in the variation between the model performance for the laboratory and field data.

- Despite the poor performance of the lab-based model using field data to predict moisture content, the field data does suggest that there should be a way to use thermal remote sensing to predict moisture content for tailings in the field, likely using a model developed using field-based data (rather than using a model developed with laboratory data).

- For full-scale industrial application of using thermal remote sensing to monitor surface moisture content, it would be more useful to collect spatially-continuous imagery over an entire impoundment so that maps can be generated to identify spatial and temporal changes in moisture content/strength, likely using UAVbased remote sensing..

- In order for UAV-based thermal remote sensing methods to be applied, the technology needs to be developed to process the thermal imagery effectively, to yield a georeferenced thermal mosaic that can be compared to ground truth data.

\subsection{References}

ASTM D2216-10, Standard Test Methods for Laboratory Determination of Water (Moisture) Content of Soil and Rock by Mass, ASTM International, West Conshohocken, PA, 2010, www.astm.org.

ASTM 4648-16, Standard Test Method for Laboratory Miniature Vane Shear Test for Saturated Fine-Grained Clayey Soil, ASTM International, West Conshohocken, PA, 2016, www.astm.org.

Bang, S. S., Bang, S., Frutiger, S., Nehl, L. M., and Comes, B. L. "Application of novel biological technique in dust suppression." Proc., Transportation Research Board 88th Annual Meeting.

Buck, S., and Gerard, D. (2001). "Cleaning Up Mining Waste." Political Economy Research Center Research Study.

Davies, M., Martin, T., and Lighthall, P. (2000). "Tailings dam stability: essential ingredients for success." Slope Stability in Surface Mining, 365-377.

Earl, R. (1997). "Prediction of trafficability and workability from soil moisture deficit." Soil and Tillage Research, 40(3), 155-168.

Greeley, R., and Iversen, J. D. (1987). Wind as a geological process: on Earth, Mars, Venus and Titan, CUP Archive. 
Liu, Z., and Zhao, Y. (2006). "Research on the method for retrieving soil moisture using thermal inertia model." Science in China Series D, 49(5), 539-545.

Minacapilli, M., Cammalleri, C., Ciraolo, G., D'Asaro, F., Iovino, M., and Maltese, A. (2012). "Thermal inertia modeling for soil surface water content estimation: A laboratory experiment." Soil Science Society of America Journal, 76(1), 92-100.

Müller, L., Lipiec, J., Kornecki, T. S., and Gebhardt, S. (2011). "Trafficability and workability of soils." Encyclopedia of Agrophysics, Springer, 912-924.

Nickling, W. G., and McKenna Neuman, C. (2009). "Aeolian sediment transport." Geomorphology of Desert Environments, Springer, 517-555.

Paul, C., and Vries, J. d. (1979). "Effect of soil water status and strength on trafficability." Canadian Journal of Soil Science, 59(3), 313-324.

Price, J. C. (1998). "Evaluation and characterization of the effectiveness of dust suppressants on iron ore mine tailings." Michigan Technological University.

Stovern, M., Betterton, E. A., Sáez, A. E., Villar, O. I. F., Rine, K. P., Russell, M. R., and King, M. (2014). "Modeling the emission, transport and deposition of contaminated dust from a mine tailing site." Reviews on environmental health, 29(1-2), 91-94.

USEPA (2012). "Particulate Matter (PM-10)." $<$ http://www.epa.gov/airtrends/aqtrnd95/pm10.html $>$.

USEPA (2014). "Fine Particule Matter (PM2.5) Designations." $<$ http://www.epa.gov/pmdesignations/>.

Vick, S. G. (1983). Planning, Design, and Analysis of Tailings Dams, John Wiley \& Sons, Inc., New York, NY.

Zwissler, B. E., Oommen, T., Vitton, S. J., and Seagren, E. A. (in review). "Thermal remote sensing for moisture content monitoring of mine tailings: laboratory study." Environmental and Engineering Geoscience. 
Chapter 5 Laboratory analysis of the generation and abatement of dust events on mine tailings in cold $^{3}$

${ }^{3}$ The material contained in this chapter is in preparation for submission to the peerreviewed literature. 


\subsection{Introduction}

Dust emissions can negatively impact humans, and has been linked to issues such as respiratory health problems, poor visibility and road conditions for drivers, and loss of agricultural soils (Bang et al. 2009). In the United States, dust emissions are regulated by the USEPA based on particle size and particle concentration. Particles of concern include coarse particles (diameters 2.5-10 $\mu \mathrm{m}$ ) and fine particles (diameters less than $2.5 \mu \mathrm{m}$ ). The latter are of particular concern because they are small enough to reach the lower portions of the human respiratory tract and can cause respiratory health problems including damage to lung tissue, cancer, and premature death (USEPA 2014). Under the Clean Air Act, the current national air quality standard limits particles smaller than 2.5 $\mu \mathrm{m}$ to $12 \mu \mathrm{g} / \mathrm{m}^{3}$ for an annual mean, or $35 \mu \mathrm{g} / \mathrm{m}^{3}$ for a 24 -hour concentration (USEPA 2014), and limits particles $2.5-10 \mu \mathrm{m}$ to $150 \mu \mathrm{g} / \mathrm{m}^{3}$ for a 24 -hour concentration (USEPA 2012).

The issue of dust emissions from soil is complex and can occur in all climates. Most commonly observed dust emissions come from warm weather conditions and especially in arid areas with minimal vegetation. Dust emission can also develop in cold climates (McKenna Neuman 1990). An important process that causes cold weather dusting is pore-ice sublimation, in which solid ice converts directly to a gas, allowing entrapped soil particles to be liberated and to become susceptible to dusting. For example, the development of Aeolian soil deposits following glaciation are believed to have been the direct result of ice sublimation, and the glacial flour dust storms in Alaska are generated through this process (Crusius et al. 2011).

Dust storms also occur on mine tailings impoundments located in cold climates. Mine tailings, the waste byproduct resulting from rock crushing and grinding in ore beneficiation, are finely crushed rock particles with an average particle size of $20 \mu \mathrm{m}$ and with a significant portion between 1 and $10 \mu \mathrm{m}$, and are deposited into permanent tailings impoundments (Vick 1983). Mine tailing impoundments are large earthen structures typically multiple square kilometers in area. A common type of tailings impoundment is a 
raised embankment design in which a main or starter dike is constructed and tailings are discharged into the impoundment. Once the tailings have reached a given a depth a second dike is constructed partly on the starter dike and partly on the tailings. This sequence is continued with additional dikes being constructed partly on the dike and partly on the tailings causing the tailings impoundment to raise upward. The benefit of raised embankment construction is that it minimizes the impoundment footprint but also allows the impoundment to be raised above the surrounding landscape.

However, due the large exposed surface area of the impoundment as well as to its elevation above the surrounding landscape, dust generation is an issue. While impoundments are susceptible to dusting during dry weather conditions, in northern climates larger dust storms can occur when freezing conditions develop. In the mining industry, dusting after a freezing event is referred to as "dry freeze" event. The term "dry freeze" comes from the process of pore-ice sublimation, causing dust to form over frozen mine tailings. In 1996 a major dust event occurred at a mine tailing impoundment in northern Michigan that generated a dust plume estimated to be over 100 kilometers long, deposited tailings on two nearby towns, and closed a US highway for over half an hour. The event occurred after four days of cold rain with freezing nights. In the morning of the dust storm, while still under overcast skies, a strong south wind developed causing temperatures to rise above freezing early in the morning. The dust storm occurred midmorning. The main cause of dust storm was sublimation of the tailings pore-ice, essentially "dry-freezing" the upper portion of the frozen tailings. The strong south wind, with sustained winds of $40 \mathrm{mph}$ and gusts up to $60 \mathrm{mph}$, resulted in causing the massive dust storm. A major factor that contributed to the massive dust storm was the formation of ice lenses during the freezing process that both concentrated and purified the water during the freezing process, thus allowing the sublimation process to accelerate. The maximum rate of sublimation occurs at the freezing point of water (Van Dijk and Law 2003), and therefore, as the temperature increases from below freezing to the freezing point, a higher rate of sublimation occurs. Above freezing, however, the pore-ice will start to melt. If winds strong enough have not developed by the time melting has started 
to cause dusting, the "freeze-dried" tailings will become saturated preventing dusting. However, from the temperature low to the freezing point, which generally occurs during the evening hours to early morning, sublimation can eliminate the near-surface ice-lenses, which has concentrated the available water in the near-surface tailings, allowing dust to form over frozen tailings and the potential for large dusting events.

To prevent a similar dust event, aggressive erosion control measures have been adopted by the mining company. These measures included water inundation (i.e., rising the water level in the impoundment to cover more tailings), mulching, utilization of paper sludge from a nearby paper mill, aerial spraying of dust suppressants, winter snow compaction (preventing spring dusting), and fencing. These controls have been effective, but are short term due to degradation of the dust suppressants and the continuous discharging of tailings into the impoundment covering the dust control efforts. The general rate of vertical tailings increase is about three feet ( 1 meter) per year. Two additional issues with dust control efforts are trafficability (Huang and Aughenbaugh 1987; Vasher 1999), or the ability of vehicles to drive over the impoundment's surface to apply dust control applications, and costs. Since a reliable model has not been developed to determine when or where dusting will develop, the mining company applies dust control to the impoundment to all accessible areas of the impoundment, adding a significant cost to tailings disposal.

Dust control research and application generally deals with "warm weather" dusting, in which a loss of water results in dust generation. Only limited research has been conducted on the issue of "cold weather" dusting, where sublimation is the driving action for dust generation on mine tailings (Vitton 1997). There has been, however, research on sublimation of pore-ice in a number of geologically related disciplines, such as Aeolian sediment transport, permafrost and periglacial landscape development (Law and Van Dijk 1994), and agricultural soil loss in northern climates (Dagesse 2011). Sublimation directly from ice has been an important aspect of glacial research. Bliss et al. (2011) examined the spatial and temporal variations of sublimation of the Taylor Glacier in Antarctica. Relevant findings from this research indicate that sublimation for example is 
about two times faster in summer than winter and that rapid sublimation occurs during storms and katabatic wind events, which is significant in studying mine tailing dust events in northern climates. And while previous studies have been conducted to attempt to understand and model ice lens generation (Konrad and Morgenstern 1980; Michalowski and Zhu 2006; Peppin and Style 2013; Reed et al. 1979; Rempel 2007; Zhang et al. 2015), sublimation (Dagesse 2011; Huang and Aughenbaugh 1987; Law and Van Dijk 1994; Lin 1981; McKenna Neuman 1990; Van Dijk and Law 2003), and dust emissions from sediments (Nickling and McKenna Neuman 2009; O'Brien and McKenna Neuman 2012; Price et al. 1997; Vasher 1999), there are no models existing that couple these processes in a way that represents the phenomenon of cold weather dusting. Due to the complexities of the process of cold weather dusting, a laboratory study would be beneficial to lead to greater understanding of the phenomenon.

Therefore, the goal of this study was to find a way to simulate the phenomenon of coldweather dusting in a laboratory setting. The objective was to develop laboratory methods to generate and quantify dust caused by sublimation-driven cold-weather dusting for mine tailings. Such methods would enable greater understanding of the mechanisms of ice lens formation, sublimation, and dust generation, and would also enable the testing of dust susceptibility and the effectiveness of dust suppressants in laboratory-scales prior to field-scale pilot studies.

\subsection{Cold Weather Dusting}

Cold weather dusting is a complex and significant contribution to dust emissions from mine tailings impoundments located in cold climates, and for other similar un-vegetated, silt-sized sediment deposits, like those in Alaska (Crusius et al. 2011). Cold weather dusting from these sediments is driven by the processes of freeze-thaw and sublimation.

Soil freezing occurs when soil loses enough heat for the pore water to freeze. Freezing occurs from the ground surface to a depth controlled by the magnitude of soil heat loss, since soil heat loss depends on the thermal properties of the soil (Gatto 1995). In general, pore water freezing leads to expansion, as water expands about nine percent 
by volume when frozen. For soils that experience freeze-thaw, the soil structure is disturbed during freezing due to the ice expansion. After thawing, this causes a higher moisture content in the soil, also resulting in a reduction in shear strength, with a 50 percent reduction in soil shear strength for some soils after a single freeze-thaw cycle (Gatto 1995). Soil freezing causes soils to be more susceptible to dusting due to lower shear strength.

However, if the pore ice sublimates prior to thawing, the soil instability is increased dramatically, and pore-ice sublimation is important process in the generation of dust in cold climates. Sublimation is a complex process by which water transitions directly from the solid phase (ice) to the gaseous phase (water vapor) without passing through the liquid phase. In fine-grained soils, sublimation is further advanced by the formation if ice lenses, which are created as the freezing front propagates downward through the soil and where capillarity is high enough for available water to be transported towards the freezing front (Gatto 1995). Silts are the soils that are the most susceptible to ice-lens formation during freeze-thaw, due to high capillarity, which draws water to the freezing front; silts also have a high enough hydraulic conductivity to allow water to move through the soil (Gatto 1995; Michalowski and Zhu 2006). Ice-lens formation then concentrates pore-ice near the surface where it is more susceptible to sublimation. The development of ice-lens in soil varies with particle-size distribution and mineralogy, though particle-size distribution is the main control for capillary action in soils during freezing. When sublimation of the pore ice and ice lenses occurs, the tailings desiccate, drying the tailings resulting in a layer of dry soil over a frozen soil layer. This allows the wind to cause the particles to become airborne more easily. The depth of sublimation determines the amount of available sediment for dusting. Sublimation at mine tailings impoundments located in cold regions occurs mainly in late fall and early spring, when there is no snow cover and sub-freezing temperatures are still causing freezing of the mine tailings. The rate and depth of sublimation is influenced by a number of variables including temperature, wind speed, relative humidity, vapor pressure gradient (a function of temperature and humidity), latent heat flux, and for ice with entrained soil, particle 
surface area and particle void ratio (Law and van Dijk 1994, Bliss et al. 2011). While there is significant understanding of the principles of sublimation, there is no accepted model that can be utilized to predict under what atmospheric conditions sublimation will occur or to what degree of soil drying will occur. In addition, models to predict freezethaw conditions as well as the magnitude of ground movement are still under development.

While previous studies have been conducted to attempt to understand and model freeze thaw (Konrad and Morgenstern 1980; Michalowski and Zhu 2006; Peppin and Style 2013; Reed et al. 1979; Rempel 2007; Zhang et al. 2015), sublimation (Dagesse 2011; Huang and Aughenbaugh 1987; Law and Van Dijk 1994; Lin 1981; McKenna Neuman 1990; Van Dijk and Law 2003), and dust emissions from sediments (Nickling and McKenna Neuman 2009; O'Brien and McKenna Neuman 2012; Price et al. 1997; Vasher 1999), there are no models existing that couple these processes in a way that represents the phenomenon of cold weather dusting, especially for fine-grained soil such as in mine tailings impoundment. Due to the complexities of the process of cold weather dusting, a laboratory study is required to understanding of the phenomenon as well as to develop controls to mitigate dusting events.

\subsection{Samples Used}

Laboratory testing was conducted using iron mine tailings samples from magnetite mines in Michigan, which are referred to as MI-mag tailings, and Minnesota, which are referred to as MN-mag tailings. These samples were blended together, which are referred to as $\mathrm{MI} / \mathrm{MN}$ blend, and this blended bulk sample was used for all laboratory testing. Samples of tailings from both impoundments, as well as the blended tailings, were characterized by specific gravity and particle-size distribution. A summary of these geotechnical properties for the tailings is presented in Figure 5-1.

The samples selected from each tailings impoundment for use in this study are primarily silt-sized, with an average size of about 20 microns, and consist mainly of quartz with little to no clay minerals. Silt-size particle size are of concern with regards to 
dust emissions and sublimation. Due to similarity in the MI-mag and MN-mag tailings samples, and due to concerns about resource limitations, MI-mag and MN-mag samples were blended to ensure enough material was available to prepare consistent samples.

To create representative samples for testing, four large tailings samples were obtained from the two tailings impoundment and were air dried for at least 2-3 weeks. During the drying process, soil aggregates that formed required crushing to liberate individual particles. This was accomplished using a Micro-Deval apparatus (ASTM D7428-15). The Micro-Deval drum was filled halfway with the dried tailings, 10 steel ball bearings were placed in the drum, and the apparatus was run for 10 minutes. Once the tailings were crushed, they were split into representative samples using a rotary sample splitter (ASTM B215-15). The splitter evenly divided each sample into 12 bins. To mix the 12 individual batches, each was passed through a mechanical splitter (ASTM C702-11) five times; the first four times, the splitter was used to mix the four individual tailings samples into a homogenous sample, while the fifth time was to split each homogenous batch into a smaller sample size. The final result of this process was 24 homogenous, blended samples of MI/MN blend tailings to be used for laboratory sample preparation, with properties shown in Figure 5-1. 


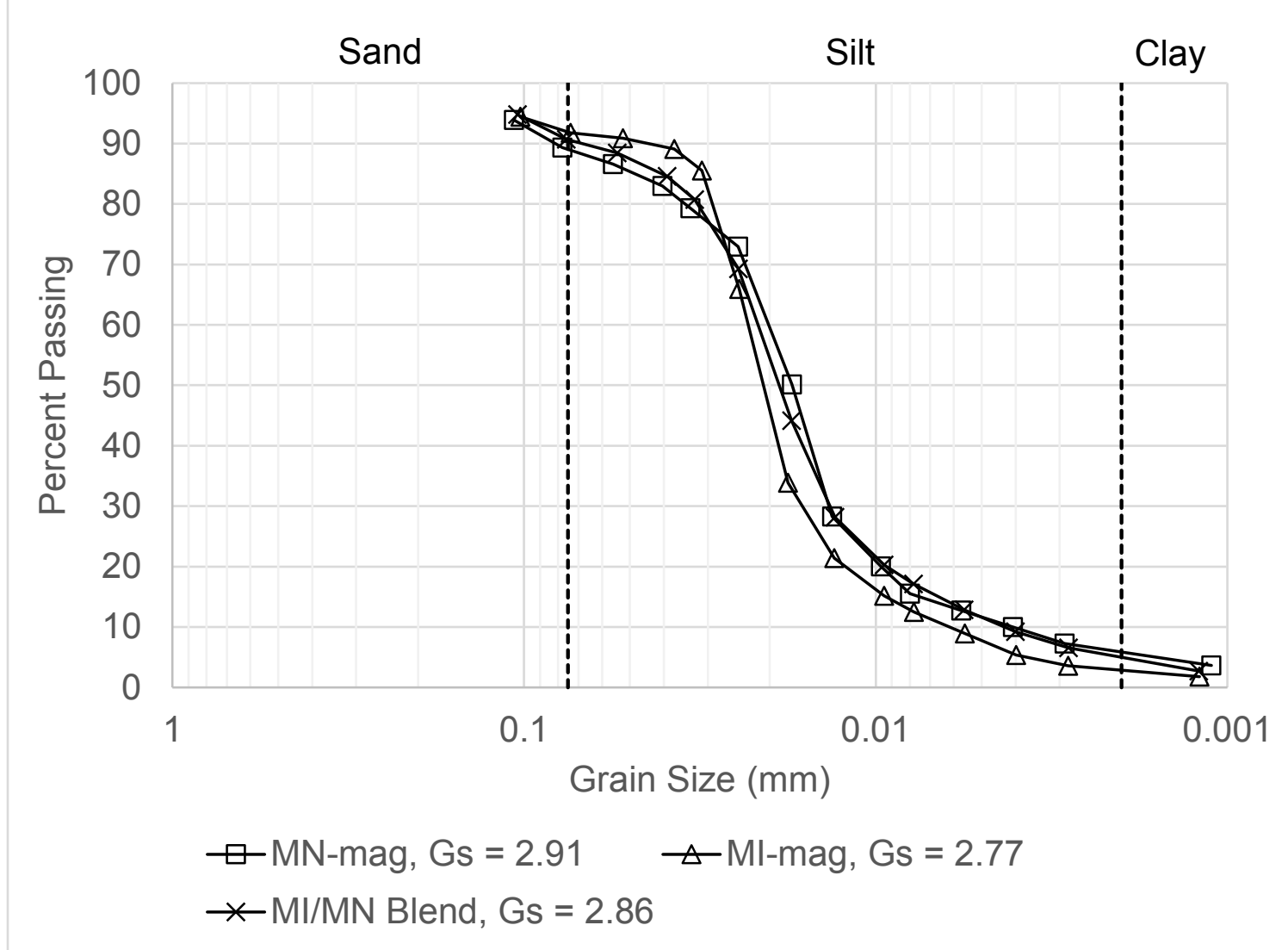

Figure 5-1 | Grain size distributions for tailings samples used in laboratory testing.

\subsection{Field Observations of Sublimation and Associated Cold Weather Dust Events}

For a laboratory study to be meaningful, it needs to represent the conditions experienced in the field. And to capture the phenomenon of cold weather dusting, those conditions are ice lens formation and sublimation of the ice, followed by wind. To observe the freezing behavior and ice lens structure of the tailings, samples were collected from the MI-mag tailings impoundment in January 2016. Photographs were taken, and the samples were transported back to the laboratory in a cooler to keep them frozen, where additional pictures were taken. Images shown in Figure 5-2 show the ice structure in the MI-mag field samples. Photographs were also taken in the field to 
document ice lens formation at the MN-mag tailings impoundment in April of 2016, as shown in Figure 5-3. The structure and random orientation of the ice lenses of the MImag and MN-mag tailings is similar. However, the MI-mag tailings (Figure 5-2) show dark tailings and ice-rich lenses, while the MN-mag tailings (Figure 5-3) show a lighter color for the tailings, and the lenses are devoid of ice. For the MN-mag tailings, both the color change and the lack of ice in the lenses suggests that sublimation has occurred, which is reasonable given that the April site visit was near the end of the winter freezing season. Anecdotally, this color change indicative of sublimation immediately precedes dust events on tailings impoundments, and watching for this sort of lightening of the tailings surface color is an indicator for tailings impoundment managers watching for potential dust events. 

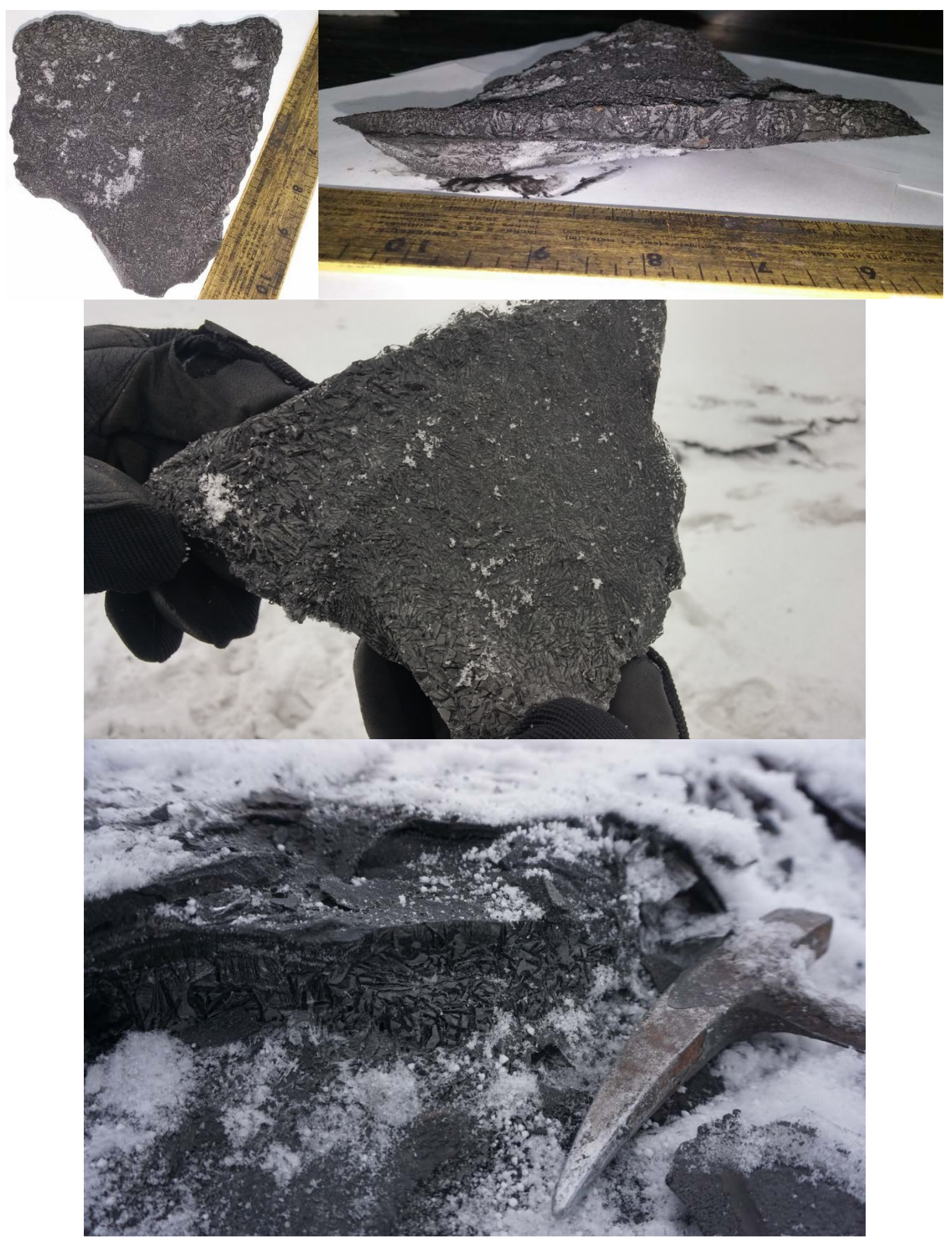

Figure 5-2 | Frozen field MI-mag tailings samples, showing ice lens formation and structure. 


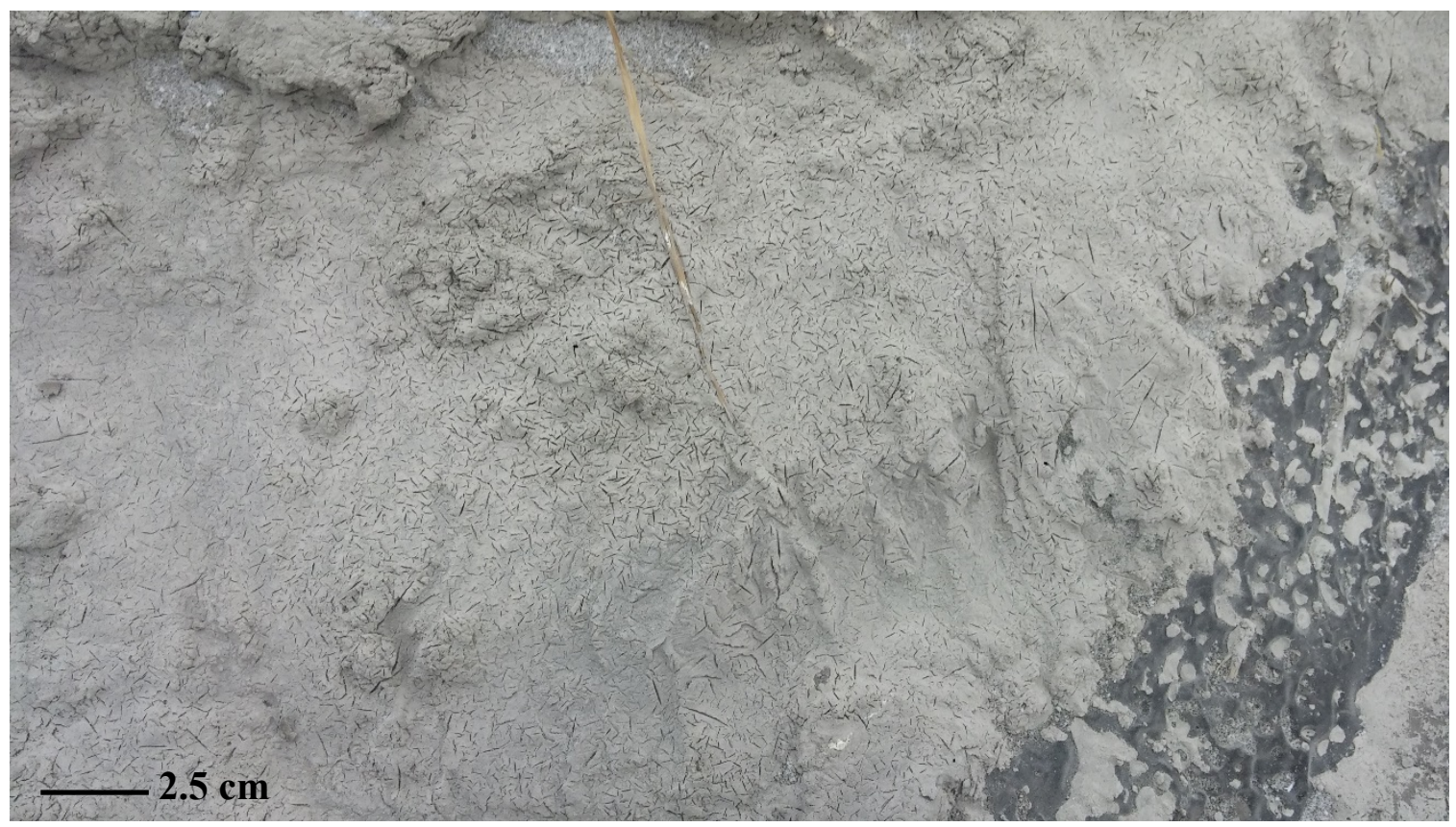

Figure 5-3 | Frozen field MN-mag tailings samples, showing ice lens formation and structure.

\subsection{Laboratory Methods to Generate Sublimation and Dust}

The goal for laboratory sample preparation was to produce samples that mimic conditions in the tailings impoundment. The tailings are deposited into the impoundments in a 55\% solid/45\% liquid slurry (Muszynski 2000; Price 1998; Vasher 1999), and the near-surface tailings gradually lose moisture from fully saturated conditions. In the case of the MI-mag tailings, in-situ moisture contents have been measured from 5\% to $30 \%$ by mass (ASTM D2216 - 10), and void ratios have been measured between 0.7 and 1.2, with a mean of 0.9 (Price 1998). These values were assumed to be representative of the conditions at the MN-mag impoundment.

Therefore, MI/MN blend laboratory samples were prepared to mimic these conditions. The technique developed, which is described in more detail by Buikema et al. (accepted), produced fully saturated samples with void ratios of 0.9 . To achieve these conditions, air dried tailings were run through a Micro-Deval apparatus (ASTM D742815) for 10 minutes to crush the large soil aggregates that form in the drying process into 
individual tailings particles. After crushing, the tailings were emptied and hand mixed under a snorkel ventilation system with distilled water to achieve a moisture content of $5 \%$ by mass for dust suppression. The moistened tailings were packed into polycarbonate soil boxes with inside dimensions of $75 \mathrm{~mm}$ x $75 \mathrm{~mm}$ x $75 \mathrm{~mm}$, with a hose barb threaded into the bottom of each box for saturation and drainage. Filter paper was placed in the bottom of the soil box and topped with a thin layer $(<1 \mathrm{~mm})$ of well-graded sand to prevent tailings from leaking out of the bottom of the soil box via the hose barb and to help facilitate capillary rise. Pluviation was used to fill the soil boxes with mine tailings using seven to 10 lifts, with each lift compacted using a hand tamp, until the soil box was full of tailings compacted to an overall void ratio of 0.9 . The soil box packing technique is summarized in the schematic presented in Figure 5-4. After filling, each soil box was saturated from the bottom upward with distilled water by connecting polypropylene tubing from a Mariotte tube constant head device to the hose barb at the base of the box, as illustrated by the schematic in Figure 5-5.

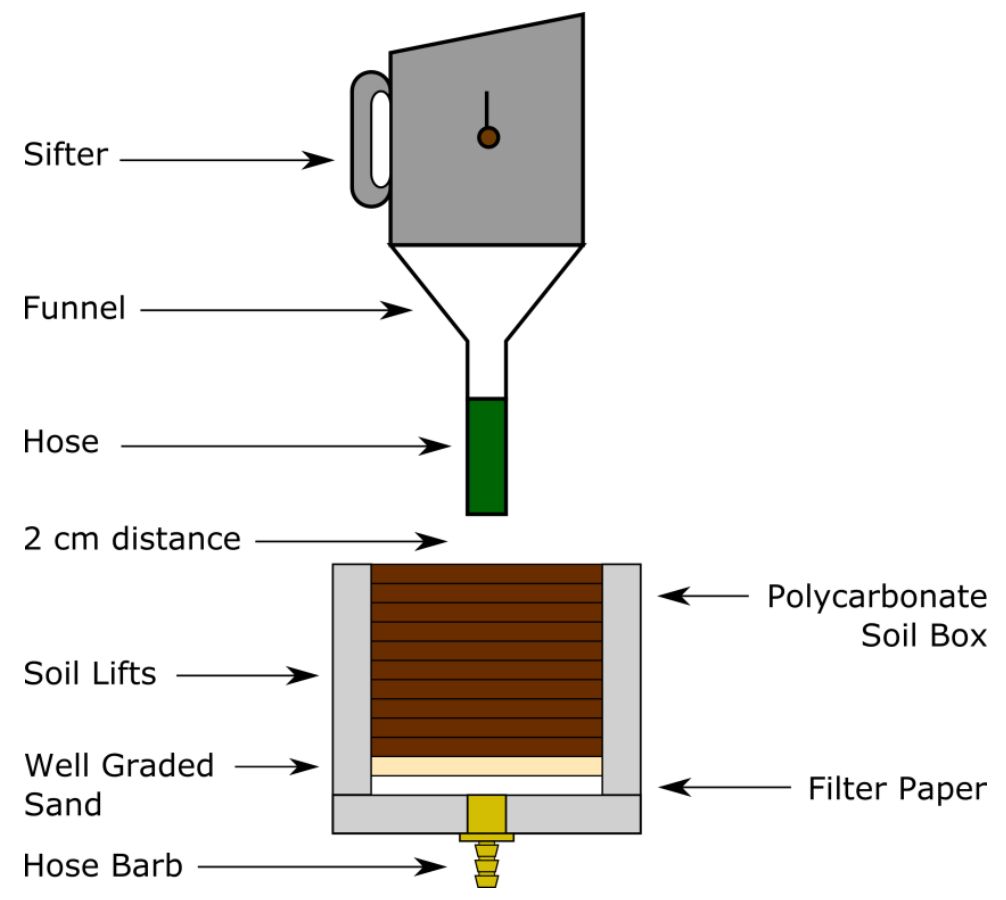

Figure 5-4 | Schematic of soil box packing technique, used in the preparation of all laboratory samples. 


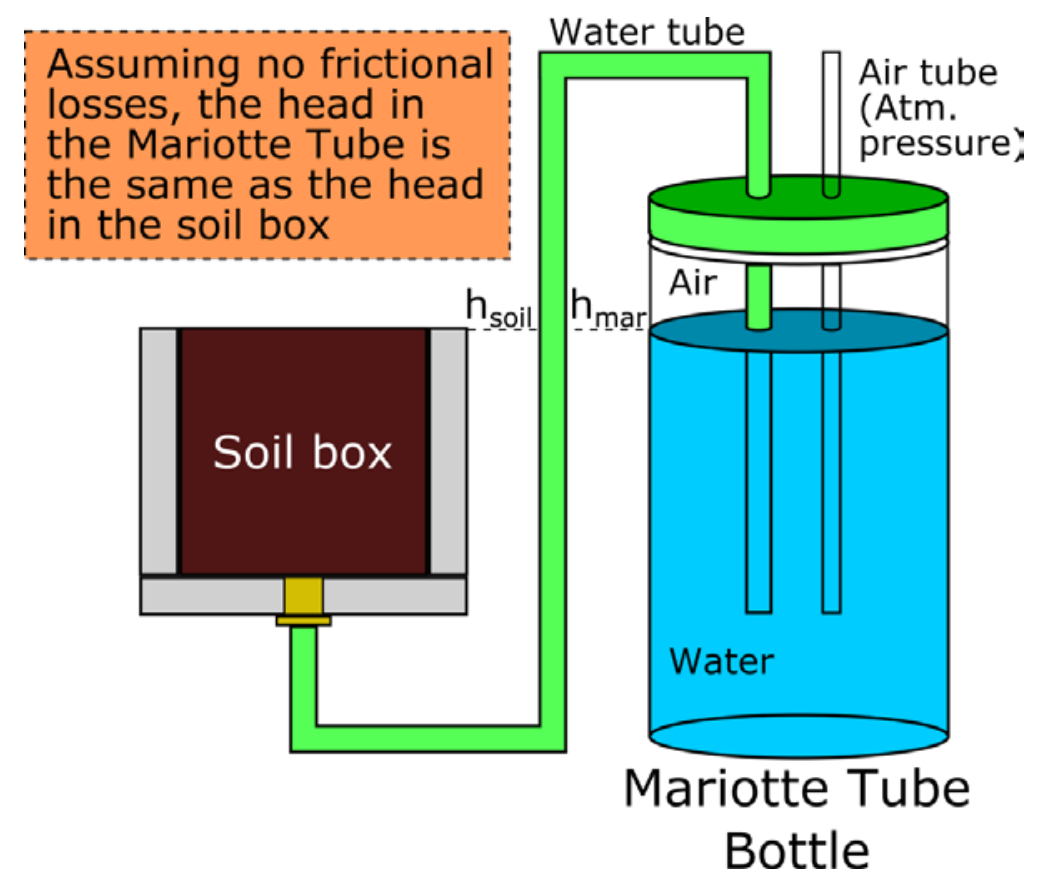

Figure 5-5 | Schematic of Mariotte Tube, used for saturation of tailings samples.

Saturated soil boxes were then placed in a controlled freezer and exposed to freezing, to generate ice lenses, and sublimation, to generate surface dust, similar to what develops on the tailings impoundments during cold weather conditions (Figure 5-2 and Figure 5-3). Methods were developed through significant trial-and-error of variables, which included freezing temperature and duration, and the presence/absence and temperature of circulating water bath below samples. It was found that generating ice lens formation similar to the ice lens formation observed in the field was difficult to replicate in the laboratory, an observation supported by Kaplar (1974). The final methods implemented in this study are described below. A summary of the measurements made before, during, and after freezing and sublimation for each sample is presented in Table 5-1.

Six soil boxes, prepared with saturated $\mathrm{MI} / \mathrm{MN}$ blend tailings, were exposed to freezing and sublimation at a time, as shown in Figure 5-6 (top view) and Figure 5-7 (front view). The saturated samples were each momentarily detached from their Mariotte tube and weighed to measure the moisture content and percent saturation for the samples prior to freezing. If the sample height was not the same as the soil box (e.g. if the sample swelled due to saturation or if the sample settled due to soil suction), the sample height 
was measured with calipers. The Mariotte tube lines were fed through a port in the side of the freezer, and reconnected to the base of each sample. Care was taken to reconnect samples to Mariotte tubes as quickly as possible, due to sample settlement and densification from increased soil suction when disconnected for more than a few minutes. Samples were then exposed to one-dimensional freezing from the surface down, as discussed by Vitton and Muszynski (2013). To accomplish this, a freezer, refrigerated recirculating chiller, and insulation were utilized. The freezer used was manufactured by Russells Technical Products (RB-3-1, serial 09982865), and featured temperature control from $-30^{\circ} \mathrm{C}$ to $80^{\circ} \mathrm{C} \pm 0.1^{\circ} \mathrm{C}$ and air circulation with dry air. There were two refrigerated recirculating chillers utilized for testing. The first was manufactured by PolyScience (5260P11A110B, serial 09982865), and features both a heating and cooling element with a working temperature range of $-10^{\circ} \mathrm{C}$ to $40^{\circ} \mathrm{C} \pm 0.1^{\circ} \mathrm{C}$ and a flow rate of $3.8 \mathrm{LPM}$. The second unit was manufactured by Neslab Insturments (RTE-4 151120, serial 81F 75894), and also features both heating and cooling elements with a working temperature range of $-30^{\circ} \mathrm{C}$ to $100^{\circ} \mathrm{C} \pm 0.1^{\circ} \mathrm{C}$ and a flow rate of $13 \mathrm{LPM}$; this second unit was used when the PolyScience unit stopped working due to pump failure.

A metal water bath (dimensions $25 \mathrm{~cm} \times 43 \mathrm{~cm}$ x $20 \mathrm{~cm}$ ) surrounded with five centimeter thick Styrofoam insulation was connected to the refrigerated recirculating chiller with $1.2 \mathrm{~cm}$ inner diameter tubing (see Figure 5-6). The water bath was placed into the freezer, and the lines connecting to the refrigerated recirculating chiller were wrapped in pipe insulation and run through the side port of the freezer. Samples were placed in the water bath onto wooden supports tall enough to allow Mariotte tube fitting and lines to extend freely from the bottom of sample boxes, as shown in Figure 5-7. It was important that a water source remained available for ice lens formation for the duration of sample freezing to continuously provide water to the freezing front. To accomplish this, the Mariotte tube bottles were kept outside of the freezer, while the water lines inside the freezing unit were insulated with $1 / 4$ " thick pipe wrap insulation to prevent freezing. Portions of each water line were also submerged in the antifreeze kept maintained at $+5^{\circ} \mathrm{C}$ from the circulating water bath to provide additional resistance to 
water line freezing. These methods were found to be sufficient to keep Mariotte tube lines from freezing. The water level in the Mariotte tube bottles was placed $5 \mathrm{~cm}$ above the tailings sample height to generate sufficient hydraulic head to allow water to flow freely to the freezing front as ice lenses formed.

Once all samples were placed into the water bath, the bath was filled with RV \& Marine antifreeze $\left(-50^{\circ} \mathrm{F}\right.$ protection) to within $1-2 \mathrm{~cm}$ of the bottom of the sample boxes, without touching the soil boxes; this was to provide additional resistance to freezing for the Mariotte tube lines inside the freezer, and to keep the bottom of the tailings samples from freezing (see Figure 5-7). Once the soil boxes were in place, the lines were insulated, and the antifreeze was in place, the void between the soil boxes and the edge of the water bath was filled with 2" Styrofoam insulation to prevent freezing of the samples from the sides. To characterize the freezing profile and to verify that one-dimensional freezing occurred, thermocouples were embedded during sample preparation for one sample of MI/MN blend tailings and placed in the bottom, middle, and top of soil boxes to monitor the temperature profile of samples during testing. Four Measurement Computing thermocouples were used, along with a USB-TC data acquisition device and DAQami 1.0 acquisition software at $1 \mathrm{~Hz}$. A schematic of the setup can be seen in Figure 5-6 (top view) and Figure 5-7 (front view).

With the Mariotte tubes connected, insulated, and set to an appropriate height, the circulating water bath filled with antifreeze, and the samples insulated on all sides but the top, the samples were ready to undergo freezing/sublimation. The refrigerated recirculating chiller was set to $+5^{\circ} \mathrm{C}$, and the freezer to $-5^{\circ} \mathrm{C}$ with dry air and air circulation turned on. After 6 hours of freezing, enough time for the surface of all samples to completely freeze, the Mariotte tube bottles were raised an extra $2.5 \mathrm{~cm}$ to ensure that the water level remained above the sample surface. The samples were monitored periodically, and the water level in each Mariotte tube bottle was marked to monitor the volume of water being drawn into the formation of ice lenses. The freezing/sublimation cycle lasted for seven days. At the conclusion of the even day freezing/sublimation cycle, samples were ready for characterization of dust emissions. 
At the end of freezing/sublimation, significant vertical expansion and dust generation occurred, so samples required extreme caution in handling while unloading from freezer and preparing for additional testing to avoid spilling of loose tailings. Insulation surrounding the samples was carefully removed, and then the samples were carefully detached from their Mariotte tube line and removed from the freezer. All testing for each sample was completed before unloading the next sample from the freezer to avoid melting. Upon sample removal, the sample mass and vertical expansion were measured. Ice lens structure and sample appearance was documented with digital photography. Each sample was then tested for erosion resistance using wind tunnel testing and/or ball drop testing. 
Table 5-1 | Description of measurements made during sample freezing and sublimation for $\mathrm{MI} / \mathrm{MN}$ blend tailings.

\begin{tabular}{|l|l|l|l|}
\hline $\begin{array}{l}\text { Data } \\
\text { Collected }\end{array}$ & $\begin{array}{l}\text { Equipment } \\
\text { Used }\end{array}$ & Purpose & $\begin{array}{l}\text { No./Timing of } \\
\text { Measurements }\end{array}$ \\
\hline Sample mass & Balance & $\begin{array}{l}\text { To monitor sample mass }+ \\
\text { changes due to ice lens } \\
\text { generation, - changes due to } \\
\text { ice sublimation) }\end{array}$ & $\begin{array}{l}\text { 1 measurement } \\
\text { before freezing, 1 } \\
\text { measurement after } \\
\text { freezing }\end{array}$ \\
\hline $\begin{array}{l}\text { Sample } \\
\text { height }\end{array}$ & Digital caliper & $\begin{array}{l}\text { To quantify volume expansion } \\
\text { due to pore ice freezing and } \\
\text { ice lens formation }\end{array}$ & $\begin{array}{l}1 \text { measurement } \\
\text { before freezing, 1 } \\
\text { measurement after } \\
\text { freezing }\end{array}$ \\
\hline $\begin{array}{l}\text { Sample } \\
\text { temperature } \\
\text { profile }\end{array}$ & Thermocouples & $\begin{array}{l}\text { To quantify the depth and rate } \\
\text { of freezing (freezing front } \\
\text { propagation, ice lens } \\
\text { formation) }\end{array}$ & $\begin{array}{l}\text { Continuous } \\
\text { measurements } \\
\text { during freezing }\end{array}$ \\
\hline $\begin{array}{l}\text { Vol. of water } \\
\text { lost from } \\
\text { Mariotte } \\
\text { tube }\end{array}$ & $\begin{array}{l}\text { Bottle with } \\
\text { marked volume } \\
\text { gradations }\end{array}$ & $\begin{array}{l}\text { To quantify volume of water } \\
\text { used to feed ice lenses }\end{array}$ & $\begin{array}{l}\text { Measurements } \\
\text { before, periodically } \\
\text { during, and after } \\
\text { freezing }\end{array}$ \\
\hline $\begin{array}{l}\text { Digital } \\
\text { pictures }\end{array}$ & Digital camera & $\begin{array}{l}\text { To document sample } \\
\text { appearance, change and ice } \\
\text { lens formation/structure }\end{array}$ & $\begin{array}{l}\text { Images before, } \\
\text { periodically during, } \\
\text { and after freezing }\end{array}$ \\
\hline
\end{tabular}




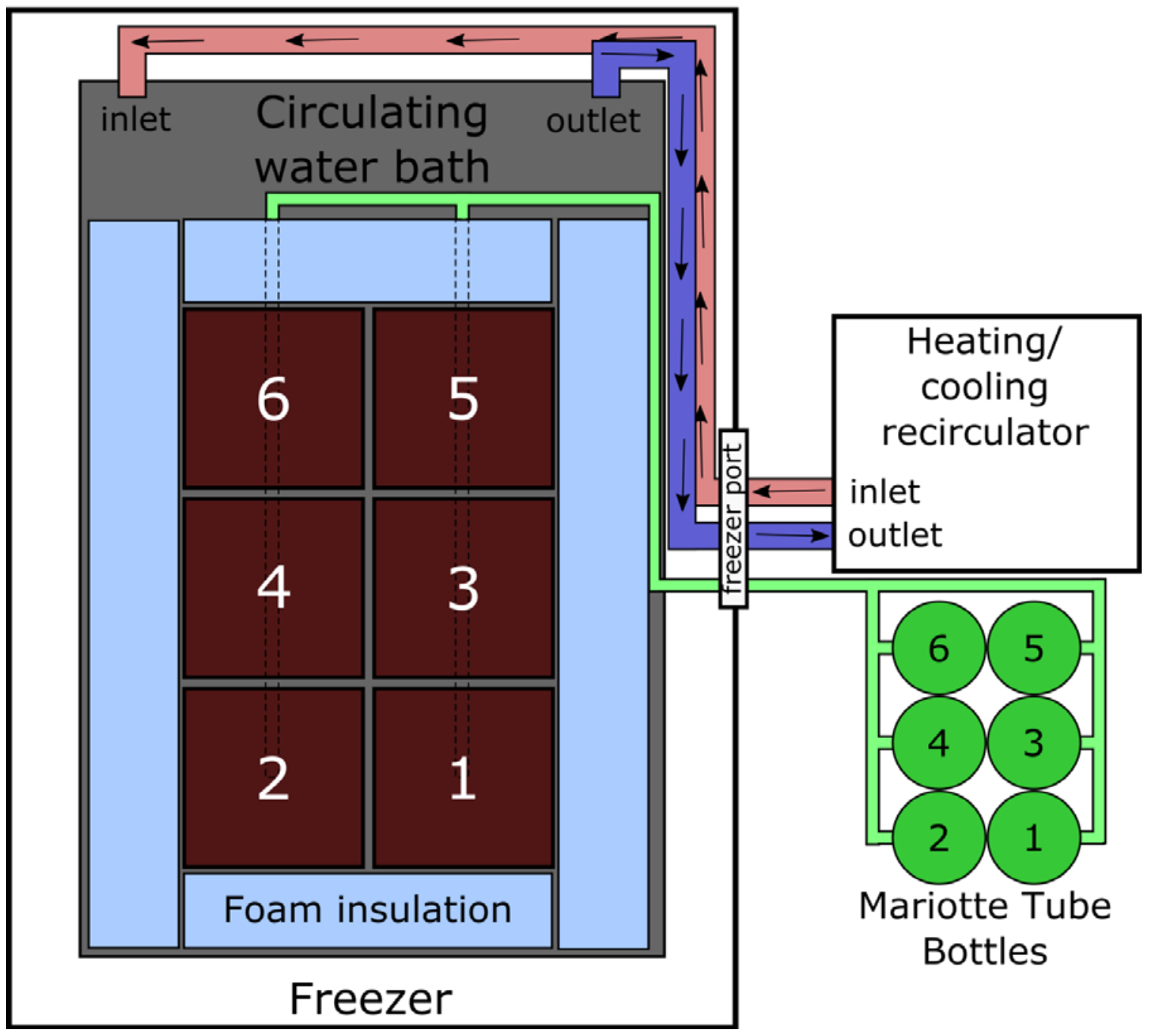

Figure 5-6 | Schematic (top view) of freezing/sublimation setup for MI-mag and MNmag samples. 


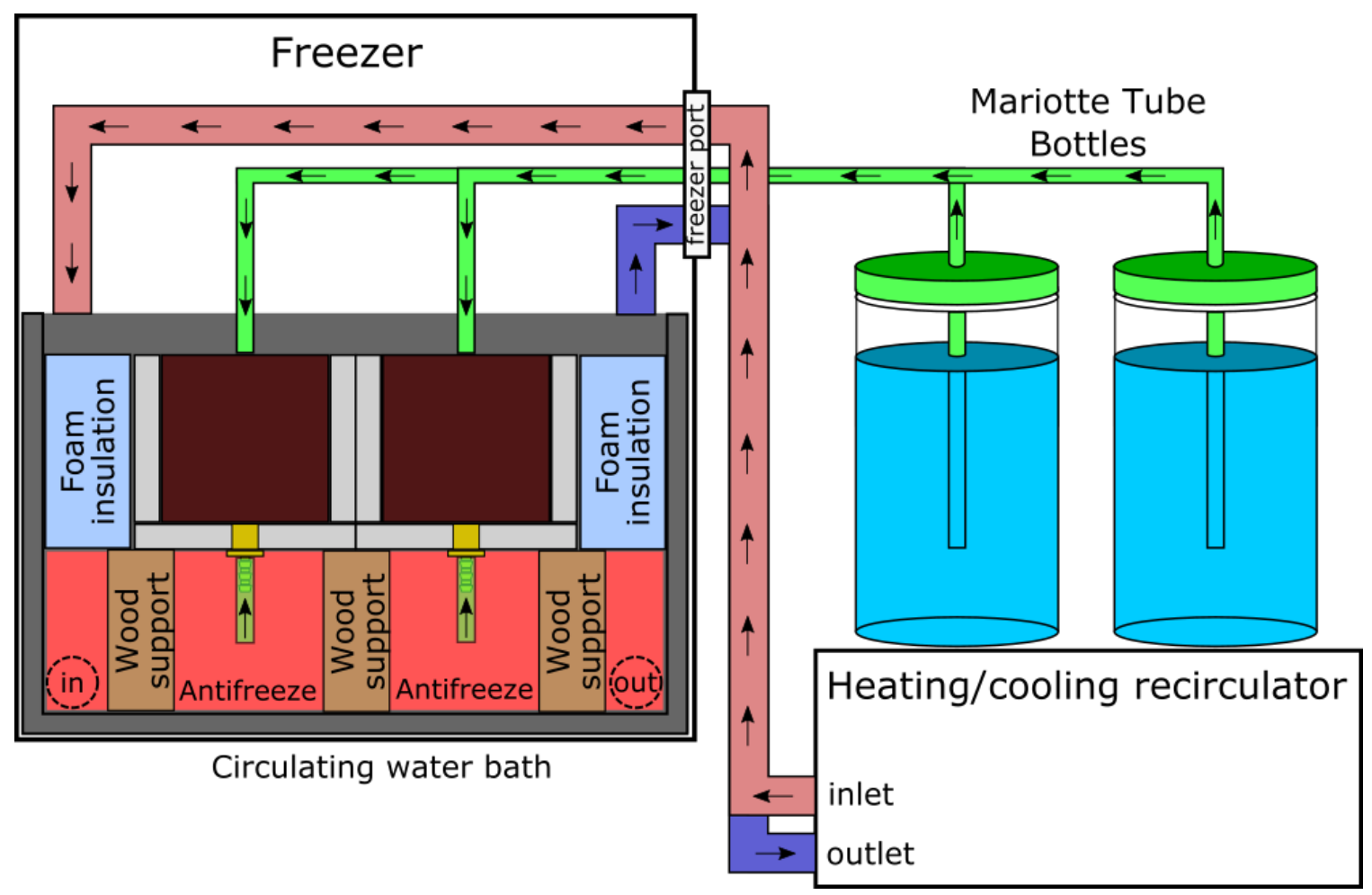

Figure 5-7 | Schematic (front view) of freezing/sublimation setup for MI-mag and MN-mag samples.

The primary method for quantifying dust emissions due to wind erosion was wind tunnel testing. The wind tunnel, shown in Figure 5-8, which was manufactured by Engineering Laboratory Design, Inc., operates with suction and is capable of reaching wind speeds of $160 \mathrm{~km} / \mathrm{hr}(100 \mathrm{mph})$, which is well above the maximum operating speed selected for this study of $96.6 \mathrm{~km} / \mathrm{hr}(60 \mathrm{mph})$, the maximum wind speed anticipated at the tailings impoundments (Price et al. 1997; Vitton 1997). The wind tunnel working section has a $30.5 \mathrm{~cm} \times 30.5 \mathrm{~cm}$ cross section, and a length of $2.4 \mathrm{~m}$. A $15.25 \mathrm{~cm} \mathrm{x} 15.25$ $\mathrm{cm}$ hole was cut in the base of the working section $1.8 \mathrm{~m}$ from the start of the working section for samples to be loaded for testing. To create a turbulent wind boundary layer in the working section, in the $1.8 \mathrm{~m}$ of working section leading up to the sample opening, 80 grit sandpaper was glued to a plywood base affixed to the polycarbonate base of the wind tunnel, as suggested by Han et al. (2009) and Okoli (2003). The 80 grit sandpaper was selected because it is a similar size to the particle sizes of tailings, in order to simulate how wind would flow over a tailings impoundment. 
The wind tunnel speed is controlled by motor frequency. In order to relate this to wind speed at the sample location, a calibration curve was developed using an Extech Pitot Tube Anemometer + Differential Manometer. For predicting wind speed at the sample's location in the working section, average wind speeds were measured over a 30 second period in the center of the front edge of the sample opening to develop the final calibration curve. Using this calibration curve and a target wind speed of $96.6 \mathrm{~km} / \mathrm{hr}$, wind tunnel tests were run at a motor frequency of $41.4 \mathrm{~Hz}$. Using this wind speed, preliminary testing was conducted to assess the effects of timing when exposing frozen samples to these wind speeds. Too little exposure to wind, and no dust was lost. Too much exposure, and the sample melted and ran down the wind tunnel, affecting mass loss measurements. Through trial and error testing, 30 seconds of testing at $96.6 \mathrm{~km} / \mathrm{hr}(41.4$ $\mathrm{Hz}$ ) was found to generate dust without allowing the samples to melt, which was an issue because the average room temperature of the wind tunnel was $21^{\circ} \mathrm{C}$.

Samples were tested in the wind tunnel by being placed along the front side and in the center of the sample hole in the base of the wind tunnel, with the sample supported by a lab jack. The sample was surrounded by a plywood plug to fill any voids between the sample box and the edge of the wind tunnel base, with the sample surface level and flush with the base of the wind tunnel. Then, the samples were exposed to 30 seconds in the wind tunnel at $96.6 \mathrm{~km} / \mathrm{hr}$. After testing, the sample was carefully removed from the wind tunnel so as to not cause sample disturbance and additional mass lost due to transport. After testing, sample mass was measured to quantify mass lost in the wind tunnel, and pictures were taken to document any visible change to the sample incurred during wind tunnel testing. A description of data collected for each sample tested in the wind tunnel is presented in Table 5-2. 


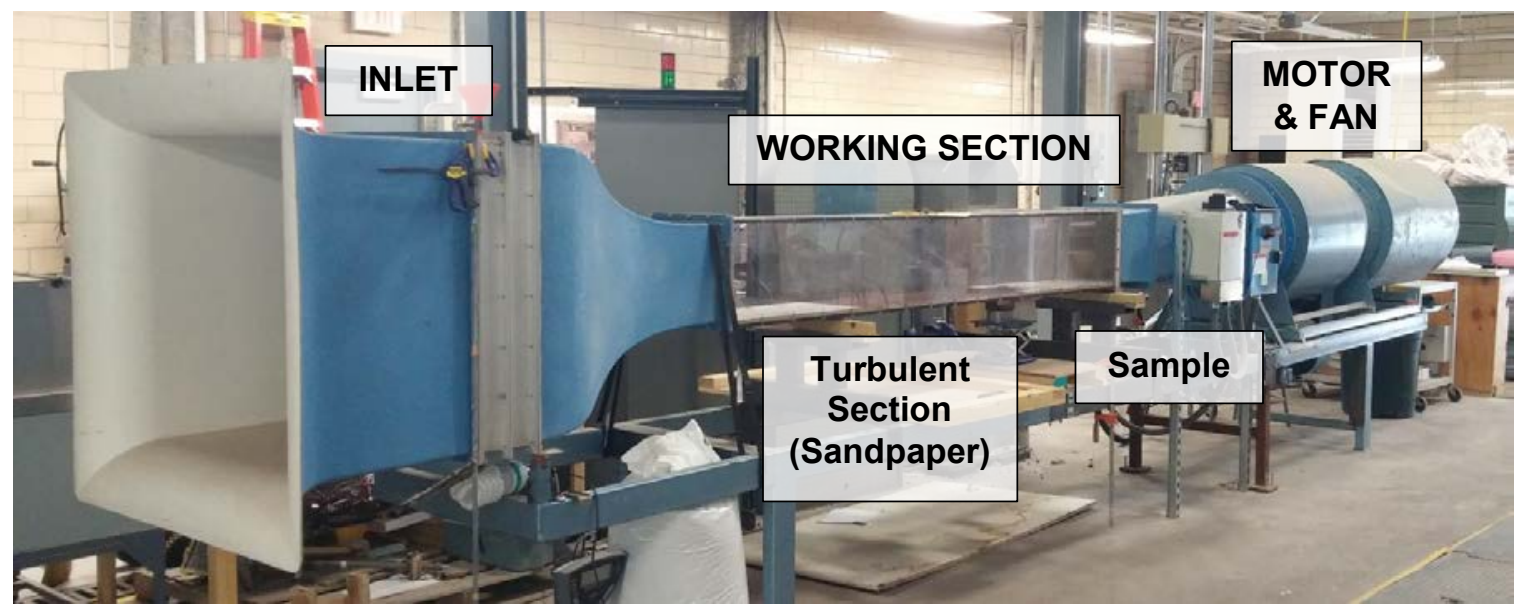

Figure 5-8 | Wind tunnel located at Michigan Technological University, Houghton, MI.

Table 5-2 | Description of measurements made during wind tunnel testing for MImag and MN-mag tailings.

\begin{tabular}{|l|l|l|l|}
\hline $\begin{array}{l}\text { Data } \\
\text { Collected }\end{array}$ & $\begin{array}{l}\text { Equipment } \\
\text { Used }\end{array}$ & Purpose & $\begin{array}{l}\text { Timing of } \\
\text { Measurements }\end{array}$ \\
\hline Sample mass & Balance & $\begin{array}{l}\text { To monitor sample mass lost } \\
\text { due to wind erosion }\end{array}$ & $\begin{array}{l}\text { Before + after wind } \\
\text { tunnel testing }\end{array}$ \\
\hline $\begin{array}{l}\text { Digital } \\
\text { pictures }\end{array}$ & $\begin{array}{l}\text { Digital } \\
\text { camera }\end{array}$ & $\begin{array}{l}\text { To document change in sample } \\
\text { color and appearance }\end{array}$ & $\begin{array}{l}\text { Before + after wind } \\
\text { tunnel testing }\end{array}$ \\
\hline Video & Video camera & $\begin{array}{l}\text { To document effects of wind on } \\
\text { sample appearance/structure }\end{array}$ & $\begin{array}{l}\text { During wind tunnel } \\
\text { testing }\end{array}$ \\
\hline
\end{tabular}

To provide continuity with an associated research project, and to provide an additional metric to assess the strength performance of tailings samples (and proposed dust suppression treatments) exposed to freezing and sublimation, a novel test developed by Buikema (2015) and Buikema et al. (2016), called the steel bearing drop test or ball drop test, was implemented. The method is discussed at depth in Buikema (2015) and is outlined below.

Ball drop testing as conducted on samples that were not visibly disturbed following wind tunnel testing. Ball drop testing was also conducted on several samples without being previously tested with the wind tunnel, and results were comparable. For each 
undisturbed sample, a $3.4 \mathrm{~g}$ steel ball bearing with a diameter of $0.945 \mathrm{~cm}$ was dropped onto the soil surface from a height of $1.5 \mathrm{~m}$. The ball bearing was removed with a magnet, and the impact crater left behind on the soil surface by the ball bearing was measured in the $\mathrm{x}$ and $\mathrm{y}$ axis using a digital caliper; these two crater diameter values were averaged together to report a single average crater diameter. This was repeated five times for each sample, with one impact crater in each quadrant of the sample surface, and one impact crater in the center of the sample surface.

\subsection{Results}

The goal for laboratory sample preparation was to yield reproducible samples that replicate field conditions as much as possible when exposed to freezing and sublimation conditions. However, no amount of quality control in the laboratory sample preparation and freezing process was able to produce samples with completely replicable freezing behavior. Despite the relatively homogenous nature of the tailings, there were too many variables involved in the test methods to achieve absolutely repeatable results. Inconsistencies that may have led to the observed variation between samples included: slight grain size variation between and within samples, variation in lift height during compaction, variation in capillary stresses due to slight variations in saturation, spatial variation in freezer position, and the time that Mariotte tubes remained unfrozen to provide water for ice lens formation and development. Still, all laboratory samples fell within acceptable moisture and density conditions and resembled the frozen tailings observed in the field in both ice lens structure and color change. Thus, all relevant variables for each sample are reported in these results.

In total, $18 \mathrm{MI} / \mathrm{MN}$ blend tailings samples were tested between March and June of 2016. All samples were prepared, frozen, and tested as described above. 16 of the MI/MN blend samples were tested using the wind tunnel, and the only samples not tested in the wind tunnel were the samples with thermocouples to avoid damage to the thermocouple system. All of the 18 samples were exposed to ball drop testing. 
A key aspect of the testing was to inspect the formation of ice lenses during the freezing and sublimation process. Thermocouple data (Figure 5-9) verified that the laboratory freezing conditions caused freezing from the tailings' surface downward, which is representative of how tailings freeze in impoundments, and is also critical to generating ice lenses. Figure 5-10 shows images of the ice lenses observed in the MI/MN blend samples. When these images of laboratory samples are compared to the ice lenses seen in the field (Figure 5-2 and Figure 5-3), it is apparent that the structure of the ice formed in the lab samples is comparable to the structure of the ice in the field samples. Thus, the laboratory methods for generating ice lenses and sublimation were deemed appropriate.

Additional qualitative observations were made during and after sample freezing and sublimation. Upon removal from the freezer, the color of the surface tailings was a notably lighter color than the underlying tailings, which is associated with sublimation. In some samples, the depth of this color change could be clearly seen from the side of the polycarbonate sample boxes. During wind tunnel testing, the color of most surface tailings lightened additionally. For the samples that had visible vertically-oriented ice lenses, when removed from the freezer, the surface ice lenses were empty, an indication that the ice had sublimated. Care was taken to not disturb these samples when removing them from the freezer because the sample surface was often visibly dusty. For samples with large amounts of vertical expansion, the sides of the upper portion of the sample where sublimation occurred were unstable because they were unsupported (see Figure 5-11); therefor, for several samples, spalling of the unsupported sides occurred in the freezer prior to removal. Typical observations of the color change seen during freezing and during wind tunnel testing are shown in Figure 5-11, and evidence of ice sublimation and the depth of sublimation achieved are documented in Figure 5-12.

The quantitative results are reported from the wind tunnel testing and ball drop testing of tailings samples. Due to the variation in the freezing behavior of samples, which can be characterized by the vertical expansion of the samples, vertical expansion is included 
in both wind tunnel results and ball drop results. Wind tunnel results are shown in Figure 5-13, and ball drop results are shown in Figure 5-14.

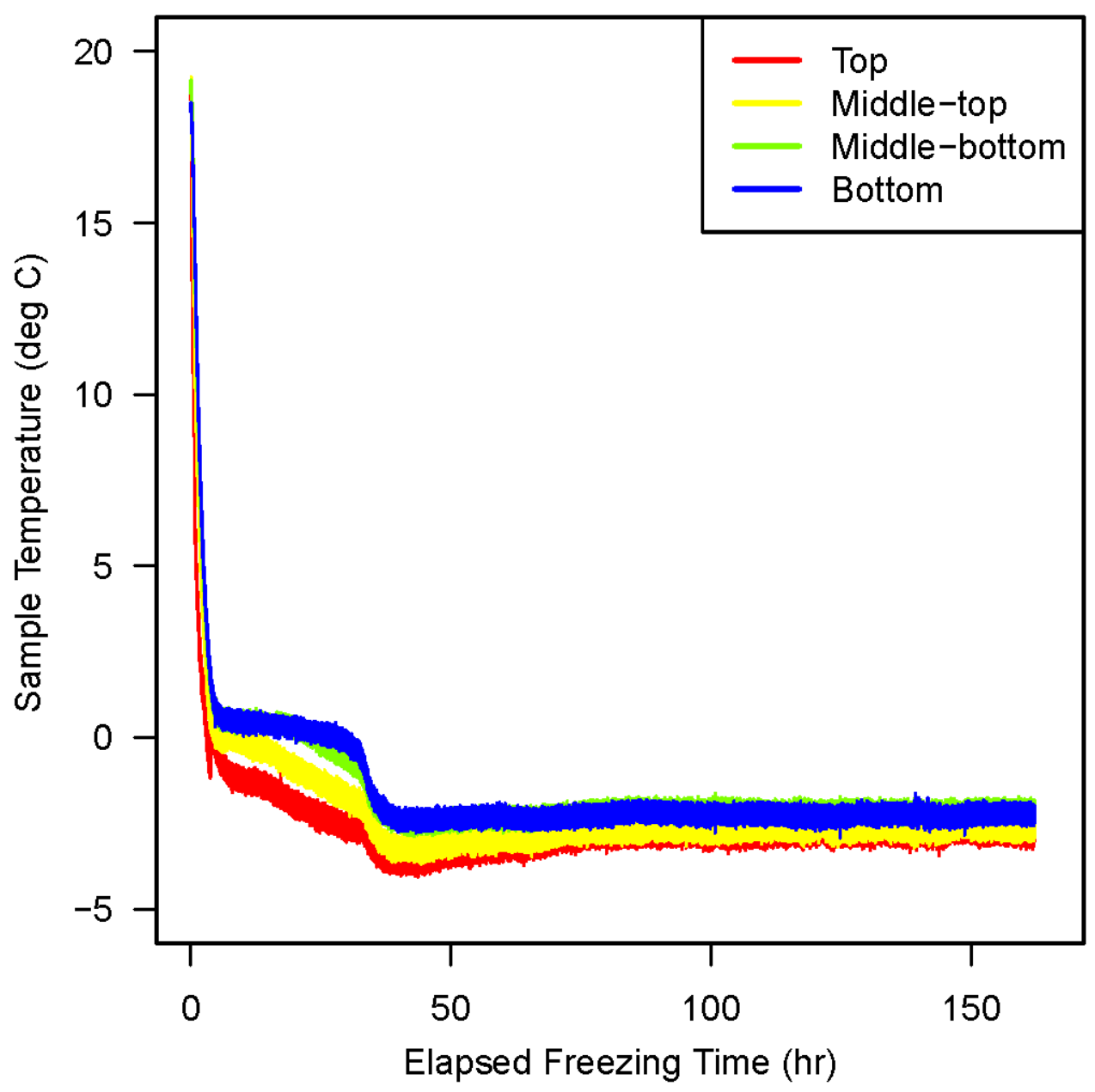

Figure 5-9 | Thermocouple data for MI/MN blend tailings sample exposed to 7 days of freezing, verifying that sample freezing occurred from the top down. 

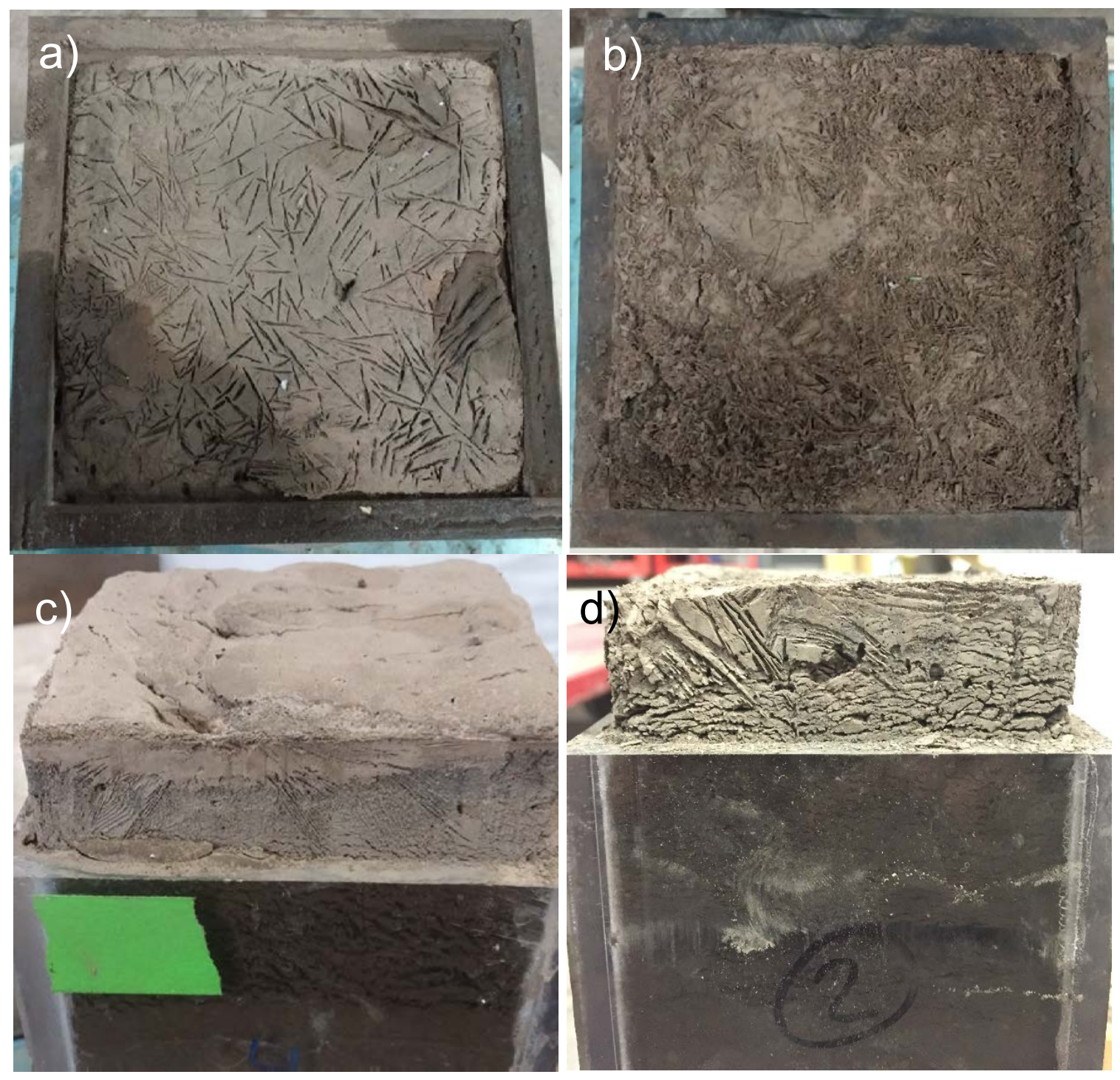

Figure 5-10 | Laboratory MI/MN blend tailings samples after freezing/sublimation, showing ice lens orientation and structure: a) and b) show vertically oriented ice lenses, and c) and d) show horizontally oriented ice lenses, both in the expanded sample above the soil box and in the tailings contained in the soil box. 

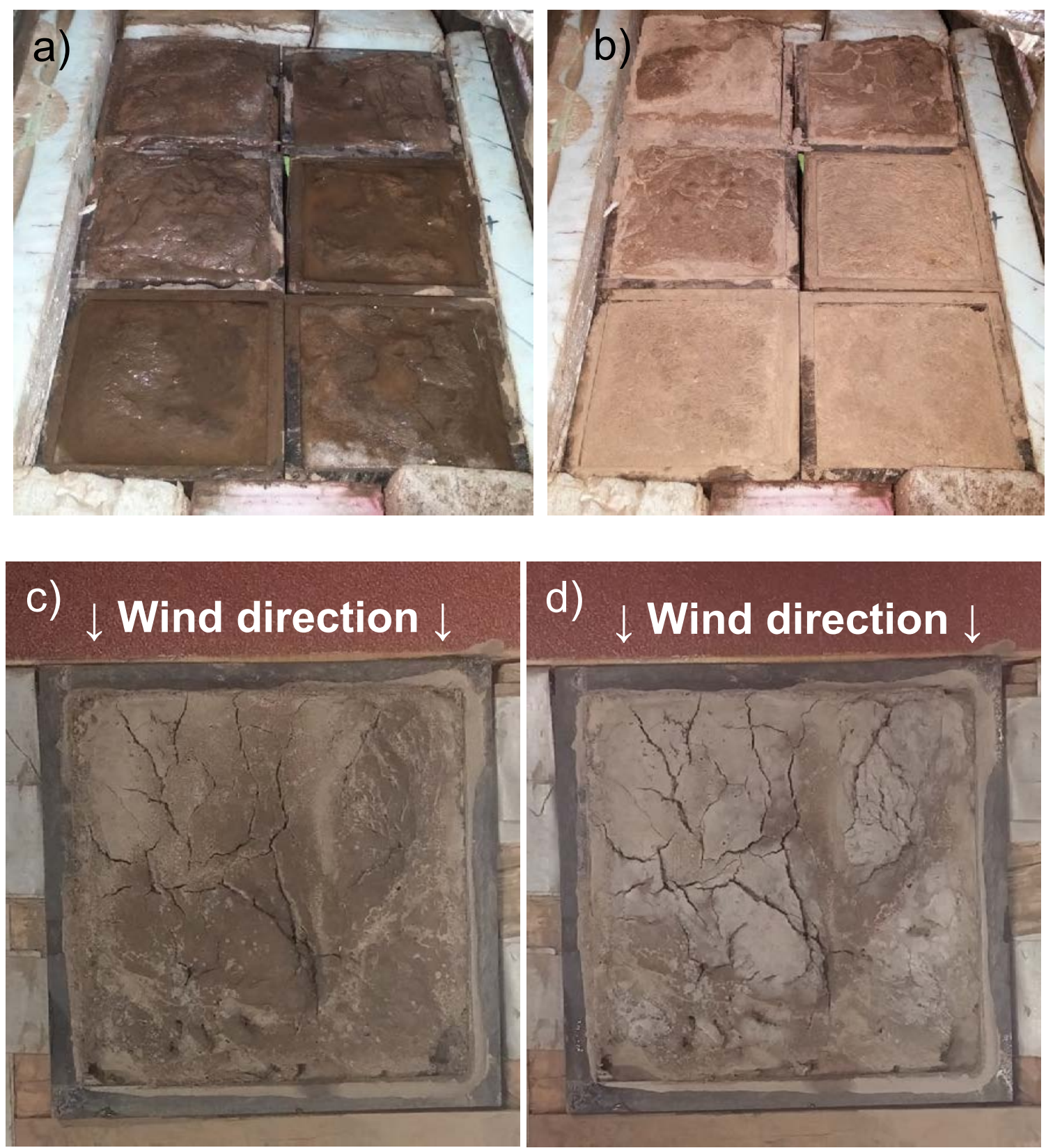

Figure 5-11 | Color change observed for MI/MN blend tailings due to sublimation in freezer and wind tunnel: a) samples prior to freezing, b) samples after 7 days of freezing, c) sample prior to wind tunnel testing, and d) sample after wind tunnel testing for 30 seconds at $97.7 \mathrm{~km} / \mathrm{hr}$. 

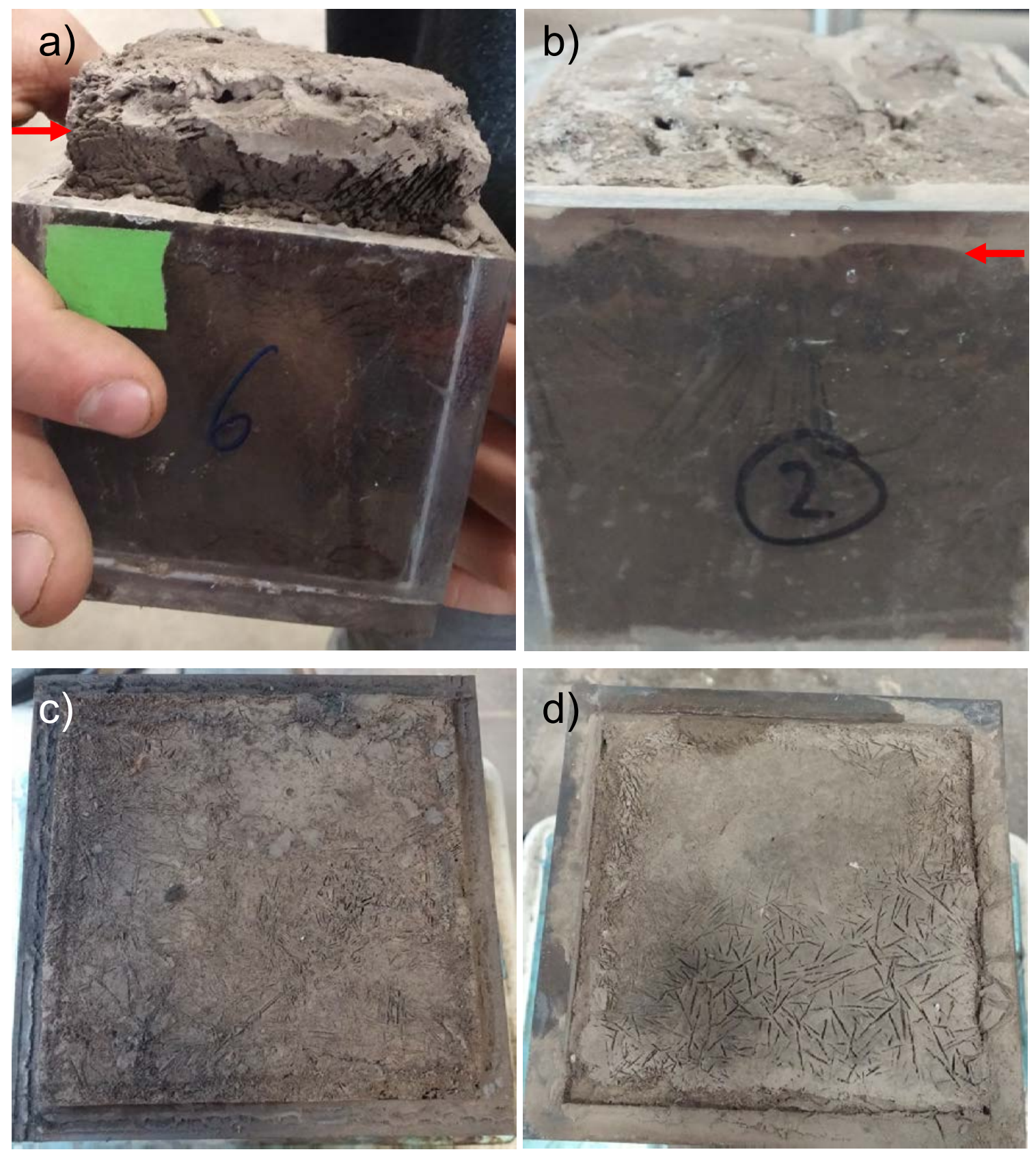

Figure 5-12 | Qualitative data supporting sublimation and dust generation for frozen MI/MN blend tailings: a) and b) light surface layer underlain by darker layer clearly shows depth of sublimation, and c) and d) surface ice lenses are empty, indicating that ice has sublimated. 


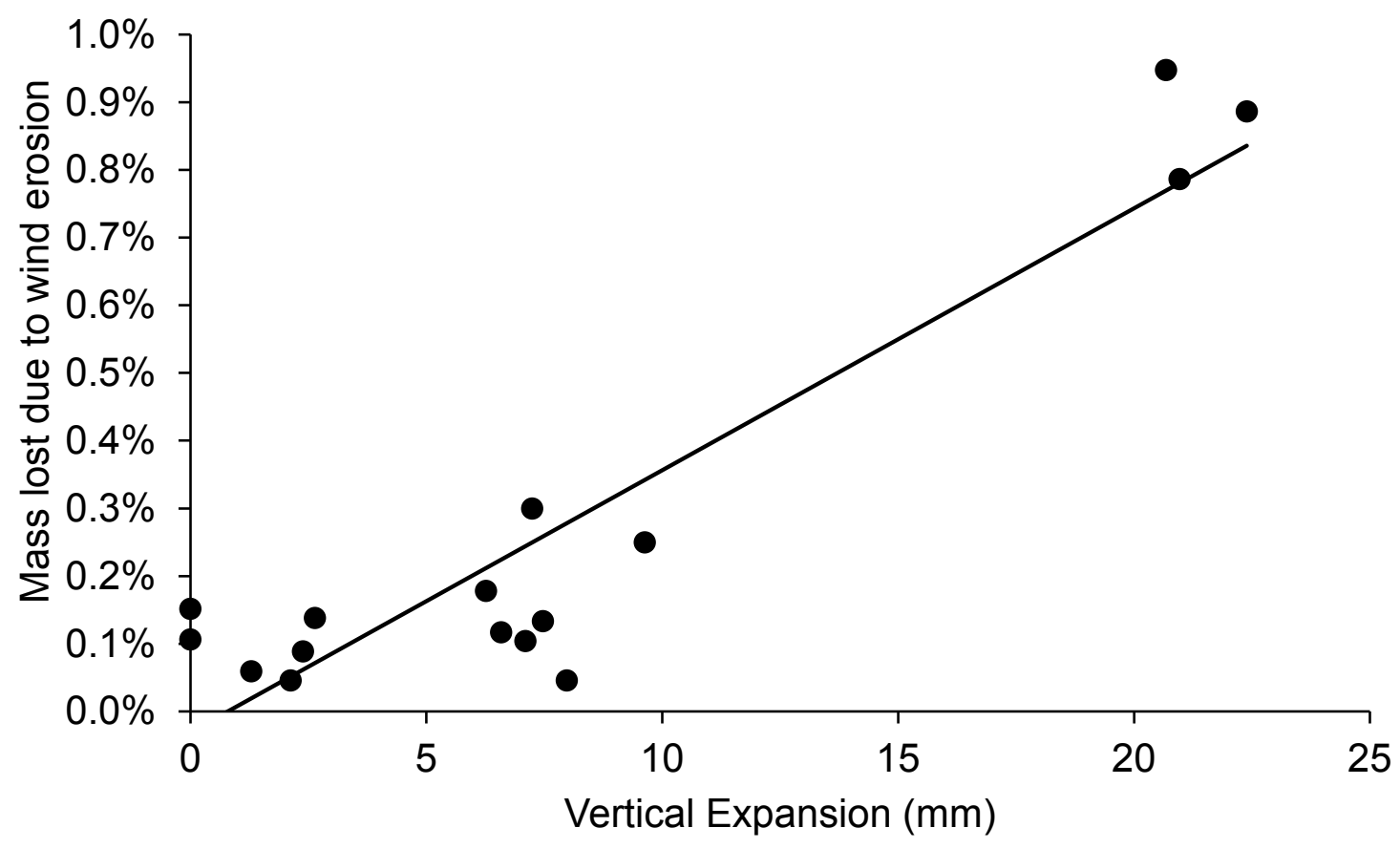

Figure 5-13 | Wind Tunnel Data for 16 MI/MN blend tailings samples, showing mass lost due to wind erosion (30 seconds at $96.6 \mathrm{~km} / \mathrm{hr}$ ) varying with sample vertical expansion $\left(\mathrm{R}^{2}=0.86\right)$.

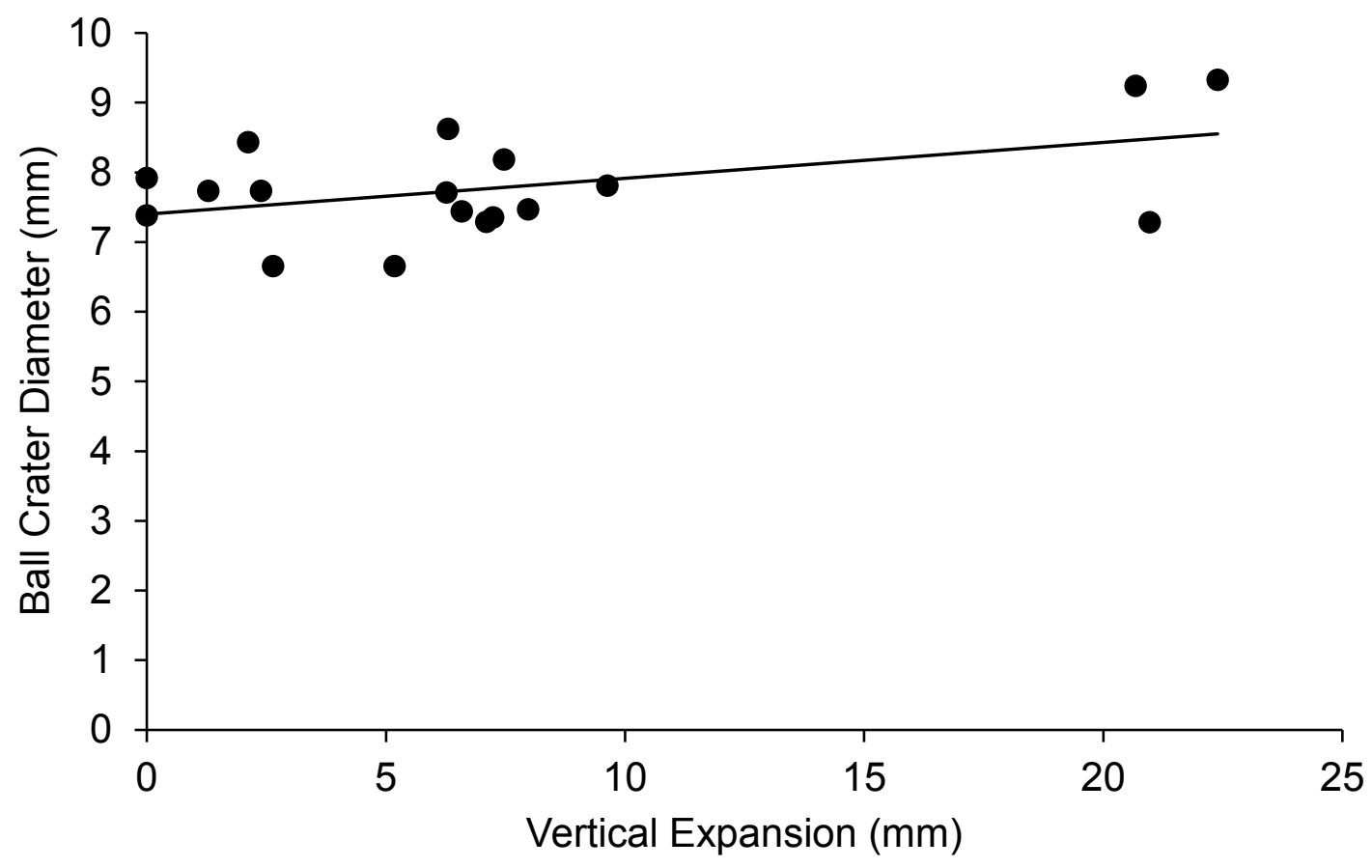

Figure 5-14 | Ball Drop Data for $18 \mathrm{MI} / \mathrm{MN}$ blend tailings samples, showing average ball crater diameter varying with sample vertical expansion $\left(\mathrm{R}^{2}=0.23\right)$. 


\subsection{Discussion}

In this study, the objective was to develop laboratory methods to generate and quantify dust generated by sublimation for mine tailings. The success of this objective was assessed both qualitatively and quantitatively.

Qualitatively, the presence of ice lenses developed during the laboratory freezing process, the similarity of the structure of these ice lenses to those seen in tailings impoundments, and the observation of a color change suggest that the laboratory methods were successful. Ice lenses shown in the laboratory samples (Figure 5-10 and Figure 5-12) are similar in size, structure, and orientation to those seen in the field samples (Figure 5-2 and Figure 5-3). The empty ice lens structures, and the lighter color of the tailings surrounding these ice lenses, shown in Figure 5-12, are also comparable to the field tailings samples observed at the end of the winter season, likely after sublimation had occurred (Figure 5-3). This color change, which is generated by the sublimation process as seen in the freezer and wind tunnel samples in Figure 5-11, is observed preceding autumn dust events on tailings impoundments and is an indicator for tailings impoundment managers of the potential for dust events. For these reasons, the laboratory methods developed and used for generating ice lenses, sublimation, and dust for the tailings samples were deemed appropriate for replicating the conditions observed at tailings impoundments.

Quantitatively, the temperature profile of the freezing samples with time and the results of the wind tunnel and ball drop testing can be used to further assess the success of these methods. The temperature profile of the freezing samples, shown in Figure 5-9, verifies that the laboratory methods used froze the tailings samples from the surface downward. This one directional freezing is representative of how tailings freeze in impoundments, and serves as further verification that laboratory methods are representative of field conditions.

The wind tunnel results, presented in Figure 5-13, show that percent mass lost from wind tunnel testing is a function of the amount of vertical expansion a sample 
experienced. This trend makes sense, because sample vertical expansion was caused entirely by ice lens formation. More ice lenses lead to more sample disturbance, especially if the ice lenses sublimate, causing more dust to be emitted. Still, it should be noted that the quantities of dust generated, as measured by percent mass lost, are small. Wind tunnel test duration was limited to 30 seconds due to concerns about sample melting. Still, some level of dust was observed on all tailings samples, and even though these methods do not yield massive quantities of dust, they do cause a similar color change to that observed in the field preceding fugitive dust events, which is indicative that the conditions in the laboratory are similar to those experienced at real tailings impoundments. Furthermore, these seemingly minor dust emissions, when scaled up from a $75 \mathrm{~mm} \times 75 \mathrm{~mm}$ surface area to the tens of square kilometer surface area of typical tailings impoundments, would still lead to significant dust events.

The ball drop results, presented in Figure 5-14, show that crater diameter may also be slightly impacted by the amount of vertical expansion a sample experienced, though to a much lesser degree than the wind tunnel results. As crude as this test may seem, the ball drop test proved to be a reliable way to capture the amount of dust generated due to sublimation.

As promising as these presented methods are, several improvements are recommended for future testing. The first recommended improvement is to use laboratory samples with a larger surface area. This will reduce the impact of disturbance to sample edges, and would provide more measurable and quantifiable dust emissions during wind tunnel testing. Even though these methods do not often yield large quantities of dust, these seemingly minimal dust emissions, when scaled up from a $75 \mathrm{~mm}$ x $75 \mathrm{~mm}$ surface area to the tens of square kilometer surface area of typical tailings impoundments, would cause significant dust events. A second recommendation is to limit the impact of sample edge disturbance by applying a sealant to the edges of the sample prior to freezing or prior to wind tunnel testing. McKenna Neuman et al. (1996) used an aerosol lacquer to successfully seal the sides of their soil samples prior to wind tunnel testing. Due to the small sample size used in this study, no sealants were used on edges so as to not alter the 
already low dust emissions from the sample. A final, but significant, recommendation is to use a wind tunnel in a laboratory able to maintain temperatures at or below freezing $\left(0^{\circ} \mathrm{C}\right)$. For wind tunnel testing in this study, the wind tunnel was located in a laboratory kept at normal room temperatures, which means that sample melting was a significant concern. All wind tunnel tests were limited to 30 seconds at $96.6 \mathrm{~km} / \mathrm{hr}$, because it was found that samples melted when exposed to longer test durations. Rather than stopping wind tunnel testing due to concerns about sample melting, keeping the wind tunnel temperature at or below freezing would enable testing duration to be extended so that all dust that could be generated by sublimation is emitted.

\subsection{Practical Applications}

These laboratory methods developed in this research were able to generate freezing and sublimation of tailings samples in a similar way to freezing and sublimation that occurs at tailings impoundments in cold climates. The development of these laboratory methods is useful to study the coupling of the phenomena of ice lens generation, sublimation, and dust emissions for fine grained sediments like mine tailings. Utilization of these methods could greatly help the understanding of these complex processes and the factors that impact dust emissions for fine grained sediments in cold climates.

Beyond the understanding of how tailings and other fine grained sediments are affected by freezing and sublimation, the application of these laboratory methods can also be used to assess the effectiveness of dust suppressants. There are a number of commercially available chemical and physical dust suppressants available, and studying how these different treatments hold up to sublimation would provide useful information to tailings impoundment managers trying to prevent cold weather dusting. Also, there is a recent push in research to develop novel dust suppressants that have a lower environmental impact (Buikema et. al 2016; Hamdan and Kavazanjian Jr 2016). In developing new dust suppressants to resist cold-weather dusting, the ability to test the treatment's resistance to sublimation at laboratory scales prior to pilot scale tests would significantly help researchers. Specifically, the effectiveness of treatments could be 
assessed by comparing mass lost due to wind erosion and ball crater diameter for treated samples to the data collected for untreated tailings (Figure 5-13 and Figure 5-14); for a measured amount of vertical expansion, if a treatment yielded a lower mass lost or a smaller ball crater diameter than the untreated tailings, it would demonstrate effective treatment.

\subsection{Summary and Conclusions}

This paper presents laboratory methods to generate and quantify dust caused by sublimation-driven cold-weather dusting for mine tailings and fine-grained soils. The laboratory methods were able to produce ice lens formation that is comparable to conditions observed in tailings impoundments in cold climates, while also successfully allowing the ice lenses to sublimate, leaving a layer of freeze-dried tailings on top of frozen tailings. This was observed by a visible color change similar to that seen at tailings impoundments. The degree of sublimation and dust susceptibility can be quantified by both wind tunnel testing and ball drop testing. These laboratory methods can be used in research to provide a better understanding of the coupling of the phenomena of ice lens generation, sublimation, and dust generation for fine grained sediments like mine tailings. These methods can also be applied to assess novel dust suppressants in development designed to resist sublimation-induced dusting, prior to pilot scale studies, to test the effectiveness of dust suppression products. 


\subsection{References}

ASTM B215-15, Standard Practices for Sampling Metal Powders, ASTM International, West Conshohocken, PA, 2015,www.astm.org.

ASTM C702 / C702M-11, Standard Practice for Reducing Samples of Aggregate to Testing Size, ASTM International, West Conshohocken, PA, 2011, www.astm.org.

ASTM D2216-10, Standard Test Methods for Laboratory Determination of Water (Moisture) Content of Soil and Rock by Mass, ASTM International, West Conshohocken, PA, 2010, www.astm.org.

ASTM D7428-15, Standard Test Method for Resistance of Fine Aggregate to Degradation by Abrasion in the Micro-Deval Apparatus, ASTM International, West Conshohocken, PA, 2015, www.astm.org.

Bang, S. S., Bang, S., Frutiger, S., Nehl, L. M., and Comes, B. L. "Application of novel biological technique in dust suppression." Proc., Transportation Research Board 88th Annual Meeting.

Bliss, A. K., Cuffey, K. M., and Kavanaugh, J. L. (2011). "Sublimation and surface energy budget of Taylor Glacier, Antarctica." Journal of Glaciology, 57(204), 684-696.

Buikema, N. D. (2015). "Stabilization of iron mine tailings through microbially induced calcite precipitation."

Buikema, N. D., Zwissler, B. E., Seagren, E. A., Oommen, T., and Vitton, S. J. (accepted). "Stabilization of Iron Mine Tailings through Biocalcification." Environmental Geotechnics.

Crusius, J., Schroth, A. W., Gasso, S., Moy, C. M., Levy, R. C., and Gatica, M. (2011). "Glacial flour dust storms in the Gulf of Alaska: Hydrologic and meteorological controls and their importance as a source of bioavailable iron." Geophysical Research Letters, 38(6).

Dagesse, D. (2011). "Effect of freeze-drying on soil aggregate stability." Soil Science Society of America Journal, 75(6), 2111-2121.

Gatto, L. W. (1995). "Soil Freeze-Thaw Effects on Bank Erodibility and Stability."US Army Corps of Engineers Cold Regions Research \& Engineering Laboratory, Hanover, NH.

Hamdan, N., and Kavazanjian Jr, E. (2016). "Enzyme-induced carbonate mineral precipitation for fugitive dust control." Géotechnique, 66(7), 546-555.

Han, Q., Qu, J., Zhang, K., Zu, R., Niu, Q., and Liao, K. (2009). "Wind tunnel investigation of the influence of surface moisture content on the entrainment and erosion of beach sand by wind using sands from tropical humid coastal southern China." Geomorphology, 104(3), 230-237.

Huang, S. L., and Aughenbaugh, N. B. (1987). "Sublimation of pore ice in frozen silt." Journal of Cold Regions Engineering, 1(4), 171-181.

Kaplar, C. W. (1974). "Freezing Test for Evaluating Relative Frost Susceptibility of Various Soils."Cold Regions Research and Engineering Lab Hanover NH. 
Konrad, J.-M., and Morgenstern, N. R. (1980). "A mechanistic theory of ice lens formation in fine-grained soils." Canadian Geotechnical Journal, 17(4), 473-486.

Law, J., and Van Dijk, D. (1994). "Sublimation as a geomorphic process: a review." Permafrost and Periglacial Processes, 5(4), 237-249.

Lin, S. (1981). "An exact solution of the sublimation problem in a porous medium." Journal of Heat Transfer, 103(1), 165-168.

McKenna Neuman, C. (1990). "Role of sublimation in particle supply for aeolian transport in cold environments." Geografiska Annaler. Series A. Physical Geography, 329-335.

McKenna Neuman, C., Maxwell, C. D., and Boulton, J. W. (1996). "Wind transport of sand surfaces crusted with photoautotrophic microorganisms." Catena, 27(3), 229-247.

Michalowski, R. L., and Zhu, M. (2006). "Frost heave modelling using porosity rate function." International journal for numerical and analytical methods in geomechanics, 30(8), 703-722.

Muszynski, M. R. (2000). "Void ratio distribution of normally consolidated coarsegrained magnetite tailings as a funcion of aging time." MS, Michigan Technological University.

Nickling, W. G., and McKenna Neuman, C. (2009). "Aeolian sediment transport." Geomorphology of Desert Environments, Springer, 517-555.

O'Brien, P., and McKenna Neuman, C. (2012). "A wind tunnel study of particle kinematics during crust rupture and erosion." Geomorphology, 173, 149-160.

Okoli, R. E. (2003). "Wind tunnel study on aeolian saltation dynamics and mass flow." Journal of arid Environments, 53(4), 569-583.

Peppin, S. S., and Style, R. W. (2013). "The physics of frost heave and ice-lens growth." Vadose Zone Journal, 12(1).

Price, J. C. (1998). "Evaluation and characterization of the effectiveness of dust suppressants on iron ore mine tailings." Michigan Technological University.

Price, J. C., Vasher, D. A., Vitton, S. J., and Paterson, K. (1997). "Characterization of fugitive dust emissions from a northern Michigan iron mine." Tailings and Mine Waste '98, Balkema Publishers, Rotterdam, Netherlands, Fort Collins, CO, 743751.

Reed, M., Lovell, C., Altschaeffl, A., and Wood, L. (1979). "Frost-heaving rate predicted from pore-size distribution." Canadian Geotechnical Journal, 16(3), 463-472.

Rempel, A. (2007). "Formation of ice lenses and frost heave." Journal of Geophysical Research: Earth Surface, 112(F2).

USEPA (2012). "Particulate Matter (PM-10)." $<$ http://www.epa.gov/airtrends/aqtrnd95/pm10.html $>$.

USEPA (2014). "Fine Particule Matter (PM2.5) Designations." $<$ http://www.epa.gov/pmdesignations/>.

USEPA (2014). "Mine Waste Technology." < http://www.epa.gov/nrmrl/std/mwt/>.

Van Dijk, D., and Law, J. (2003). "The rate of grain release by pore-ice sublimation in cold-aeolian environments." Geografiska Annaler: Series A, Physical Geography, 85(1), 99-113. 
Vasher, D. R. (1999). "A technique for assessing fugitive particulate emission potential from an active mine tailings basin." Michigan Technological University.

Vick, S. G. (1983). Planning, Design, and Analysis of Tailings Dams, John Wiley \& Sons, Inc., New York, NY.

Vitton, S. (1997). "Environmental and operational considerations in the use of paper waste sludge for dust control on iron ore mine tailings." First International Workshop on the Use of Paper Industry Sludges in Environmental Geotechnology and ConstructionHelsinki, Finland.

Zhang, S., Sheng, D., Zhao, G., Niu, F., and He, Z. (2015). "Analysis of frost heave mechanisms in a high-speed railway embankment." Canadian Geotechnical Journal, 53(3), 520-529. 
Chapter 6 Biological Soil Crust monitoring and laboratory testing to explore dust suppression potential for mine tailings ${ }^{4}$

${ }^{4}$ The material contained in this chapter is in preparation for submission to the peerreviewed literature. 


\subsection{Introduction}

Fugitive dust emissions from mine tailings impoundments are a major environmental concern for the mining industry. Mine tailings are the waste product resulting from ore beneficiation, in which the crushing and grinding operations required to separate the ore from the rock yield finely crushed rock particles with an average particle size of $20 \mu \mathrm{m}$ and a significant portion of particles between 1 and $10 \mu \mathrm{m}$ in diameter. The tailings are often deposited in slurry form into permanent tailings impoundments, which are among the largest earthen structures in the world (Vick 1983). Due to the small size of the tailings particles and the large volume of tailings stored in impoundments, air pollution from blowing dust is a significant concern to the environment (Buck and Gerard 2001; Stovern et al. 2014), and human health (Bang et al. 2009). Accordingly, these dust emissions are regulated by the USEPA under the Clean Air Act as airborne particulate matter based on particle size and particle concentration (USEPA 2012; USEPA 2014).

The emission of dust from soil is a complex phenomenon in both warm and cold climates. However, for cold climates, the phenomenon of cold weather dusting is an additional complicating factor and a significant contribution to dust emissions (McKenna Neuman 1990). Cold weather dusting is driven by the processes of freeze-thaw and sublimation, which leave behind a surface layer of loose, dry, and unstable tailings that can become airborne and generate dust at much lower wind speeds than required for the original tailings material.

Tailings impoundment managers actively monitor for dust emissions, and apply dust suppression treatments to the surface of tailings impoundments to limit dust emissions. To be successful, any attempts to mitigate dusting on tailings impoundments must either increase the surface shear strength of the tailings or decrease the force of the wind acting on the tailings. Most mitigation techniques focus on increasing the strength of the tailings, because the speed and direction of the wind are so variable and are for the most part out of human control. There are many mitigation techniques that are currently used at tailings impoundments to attempt to increase the surface strength of the tailings, and they rely on chemical or physical additives (Bang et al. 2009), including water 
inundation, sludge cover, mulching cover, and crusting or binding agents. However, many of these physical and chemical treatments have environmental consequences, have high costs for application, and may lose effectiveness with time due to factors like UV degradation (Bang et al. 2009). Therefore, a more effective, economical, and longer lasting solution without environmental consequences is desirable.

Several research groups are investigating the use of microbial soil stabilization as an alternative to chemical and physical treatments to increase surface strength and resist soil erosion. The most common process uses a bacterium, Sporosarcina pasteurii, in a process called microbially induced calcite precipitation, or MICP (Bang and Bang 2011; Bang et al. 2009; Buikema et al. accepted; Meyer et al. 2011). In most studies, MICP requires the addition of urea and calcium. The majority of research concerning the use of MICP for surficial strength increase has been tested on sand samples at laboratory scales, and only one study has investigated MICP for mine tailings (Buikema et al. accepted). Laboratory testing has shown an increase in surface strength and reduced erosion susceptibility, but scaling MICP to field applications would have to address issues

In most studies, MICP has been applied by promoting urea degradation, or ureolysis, by a bacterium such as Sporosarcina pasteurii. To promote ureolysis, the bacteria are provided urea, which is hydrolyzed producing ammonia and bicarbonate. The ammonia production results in an increase in $\mathrm{pH}$, shifting the bicarbonate to the carbonate form, which, in the presence of excess calcium, will precipitate as calcium carbonate. The majority of research on MICP for surficial strength increase and dusting control has been tested on sand samples at laboratory scales (Bang and Bang 2011; Bang et al. 2009; Meyer et al. 2011), and only one study to-date has investigated MICP for mine tailings (Buikema et al. accepted). Laboratory testing has shown an increase in surface strength and reduced erosion susceptibility, but scaling MICP to field applications will require addressing issues such as cost, growth time, uniformity of bacteria/nutrient application, and longevity (DeJong et al. 2013).

An alternative biomediated approach for erosion control is to take inspiration from one of nature's most successful solutions to erosion resistance, biological soil crusts 
(BSCs). BSCs are naturally occurring and cover otherwise bare soil, providing an increased surface strength and resistance to soil erosion. BSCs have been proven to reduce wind and water erosion in natural systems (Eldridge and Greene 1994). Soil featuring biological crusts that are well-developed has been shown to be 2 to 130 times more resistant to soil erosion than bare soil or soil with crusts that are not fully developed (Belnap and Gillette 1997; Belnap et al. 2001; Leys and Eldridge 1998; McKenna Neuman et al. 1996; Williams et al. 1995). If reductions in soil erosion of this magnitude have been documented for natural systems, it indicates that it may be possible for similar results to be obtained from human stimulation of BSCs in other locations that are susceptible to erosion. In fact, the growth of BSCs on previously un-crusted soil has been demonstrated successfully in the laboratory (Liu et al. 2008; McKenna Neuman and Maxwell 1999; McKenna Neuman and Maxwell 2002; McKenna Neuman et al. 2005; McKenna Neuman et al. 1996; O'Brien and McKenna Neuman 2012). However, while BSCs colonize and grow on previously bare soil, with or without human intervention, it is uncertain if they can be cultivated on iron mine tailings impoundments and used to suppress dusting.

The overall goal of this study was to assess if BSCs are a viable dust suppressant treatment for iron mine tailings. Specific objectives for this research were as follows: (1) assess if BSCs be grown on iron mine tailings, and (2) evaluate the potential for BSCs to be used for decreasing dust emissions from iron mine tailings that experience coldweather dusting. Tools used in determining if BSCs can grow, withstand freezing, resist wind erosion, and increase surface strength include remote sensing monitoring of crust growth, biomass characterization, scanning electron microscopy, wind tunnel testing, and ball drop strength testing.

\subsection{Background}

To apply BSCs for erosion control, it is important to understand their characteristics. BSCs are a specialized community of macro- and micro-biological components on or near the surface of soil (Harper and Marble 1988; West 1990). The components of BSCs 
can vary, but often include cyanobacteria, (green) algae, lichen, bryophytes including mosses and liverworts, microfungi, and bacteria (Belnap and Gillette 1998; West 1990). BSCs can develop on the ground surface anywhere that vascular plant growth is limited or absent, but they are especially prevalent in hot or cold arid regions (Cameron and Blank 1966; Friedmann and Galun 1974; Friedmann and Ocampo-Paus 1976; Harper and Marble 1988; West 1990). They are most commonly found in desert settings, but have been found in a wide variety of locations (Belnap et al. 2001), including more humid regions (Meyer et al. 2011; Thiet et al. 2005). BSCs have been observed on abandoned mine tailings, such as coal tailings in New Mexico, Arizona, and North Dakota (Shubert and Starks 1980), copper tailings in China (Huang et al. 2011; Sun et al. 2004), brown coal and lignite tailings in Central Europe (Lukešová 2001), and gold tailings in South Africa (Orlekowsky et al. 2013).

BSCs cover otherwise bare soil, providing an increased surface strength and resistance to wind and water erosion, as noted above. However, because BSCs can be composed of any combination of cyanobacteria, algae, lichen, bryophytes, microfungi, and bacteria, some crusts are stronger and more effective at resisting erosion than others. Moss crusts provide the most resistance from water and wind erosion, followed by lichen crusts, cyanobacterial/algal crusts, and bare soil (Belnap 1993; Belnap and Gardner 1993; Belnap and Gillette 1997; Belnap and Gillette 1998; Belnap et al. 2001). These differences in erosion resistance are due to the different crust components acting to resist erosion in different ways. Cyanobacteria and green algae excrete sticky polysaccharides, which binds soil particles together and creates larger soil aggregates that are more resistant to wind and water erosion. Similarly, lichen and moss rhizines bind soil particles together and form larger soil aggregates. Lichen, bryophytes, microfungi, and other crusts with higher surface profiles create a rough microtopography, which causes a still-air boundary layer to develop that protects the ground surface from wind erosion.

Although cyanobacterial crusts have lower strength than many other biological soil crusts, they are still good candidates for increasing erosion resistance. For example, filamentous cyanobacteria soil crusts have many beneficial characteristics. In addition to 
the soil particle binding that occurs from the sticky polysaccharide sheaths that cyanobacteria produce (also known as extracellular polymeric substances or EPS), cyanobacterial filaments grow between soil particles in the upper few millimeters of soil, forming a matrix that binds the soil together and holds them in place, protecting them from erosion forces (Belnap 1993; Belnap and Gardner 1993; Belnap and Gillette 1997; Cameron and Blank 1966; Friedmann and Galun 1974; Friedmann and Ocampo-Paus 1976; McKenna Neuman et al. 1996; Williams et al. 1995). Soil particles can also be attached to sites along the cyanobacteria cell wall (Eldridge and Leys 2003). Because of the soil matrix that is formed with the polysaccharide sheaths and filaments, filamentous cyanobacterial crusts can still provide significant protection from the abrasion of windborne particles (McKenna Neuman et al. 1996).

Another benefit of the establishment of BSCs composed of filamentous cyanobacteria is that cyanobacteria, along with green algae, are considered to be pioneer organisms for other natural crust components (Thiet et al. 2005). Large filamentous cyanobacteria are usually the first photosynthetic species to grow on bare or disturbed soils (Ashley and Rushforth 1984; Belnap 1995; Belnap et al. 2001). This role can be attributed to their ability to colonize surfaces from the air and their ability to move across soil surfaces due to growth and/or motility (Schlichting 1969). The pioneer cyanobacteria form early successional crusts upon which the other biological soil crust components can grow (Thiet et al. 2005).

Most studies of BSCs to date have assessed naturally occurring crusts in-situ (Pérez 1997; Thomas and Dougill 2007; Zaady et al. 2010), but there has been some work focused on demonstrating laboratory growth of BSCs on previously un-crusted soil (Liu et al. 2008; McKenna Neuman and Maxwell 1999; McKenna Neuman and Maxwell 2002; McKenna Neuman et al. 2005; McKenna Neuman et al. 1996; O'Brien and McKenna Neuman 2012). Thus, there is potential for growth of BSCs on laboratory and field samples of mine tailings, assuming that: (1) a suitable pioneer organism is selected, and (2) the field crusts can develop sufficiently fast between tailings discharges to adequately increase surface strength. Field application would also need to consider 
sample disturbance, as disturbance of a biological soil crust via compressional loading (like foot traffic and vehicular loading) can greatly reduce its ability to resist erosion (Belnap and Gillette 1998), and full recovery can require time on the order of magnitude of years to decades to even centuries (Belnap and Eldridge 2003).

\subsection{Tailings Samples Used}

All laboratory testing was conducted with samples of tailings obtained from iron mine tailings impoundments in the region. Specifically, tailings from a Michigan hematite mine (called MI-hem tailings), a Michigan magnetite mine (called MI-mag tailings) and a Minnesota magnetite mine (called MN-mag tailings) were used. In addition, due to material limitations, MI-mag and MN-mag samples were blended (called MI/MN blend) to ensure enough material was available to prepare consistent samples. Geotechnical characterizations for each type of tailings, as well as a description of the blending procedure used, is provided in Chapter 5 and Zwissler et al. (in preparation).

\subsection{Proof-of-Concept Growth of BSCs on tailings}

Prior to doing extensive tests on the effects of biological treatments on tailings, proof of concept testing was conducted to verify that BSCs could be grown on iron mine tailings. For this demonstration, putative cyanobacterial inoculum cultures were used that had been isolated from native BSCs from the Upper Peninsula of Michigan. Biological soil crust samples were collected on November 4, 2012 from the Black Creek Nature Sanctuary and on June 28, 2014 from the Eagle River beach. Crust samples were aseptically transferred to petri dishes containing MI-mag and MI-hem tailings saturated with BG-11 growth medium (Stanier et al. 1971) by breaking up the crust by hand or with a sterile mortar and pestle, and sprinkling the crust over the tailings surface. Approximately 1 gram of crust was transferred to every plate. The petri dish samples were subsequently moistened with water once per week, and given a dose of BG-11 medium once per week. The samples were maintained in a Conviron BDR16 growth chamber $\left(20^{\circ} \mathrm{C}\right.$ with full spectrum lights) until September 2013 , after which they were maintained at room temperature next to an exterior window for a source of natural light. 
Growth was monitored visually. Notable crust growth and development occurred on MImag and MI-hem tailings after four to six months of maintenance, as shown in Figure 6-1. A variety of organisms, ranging from putative cyanobacteria to higher organisms, were observed. This successfully proved that BSCs can grow on iron mine tailings. 

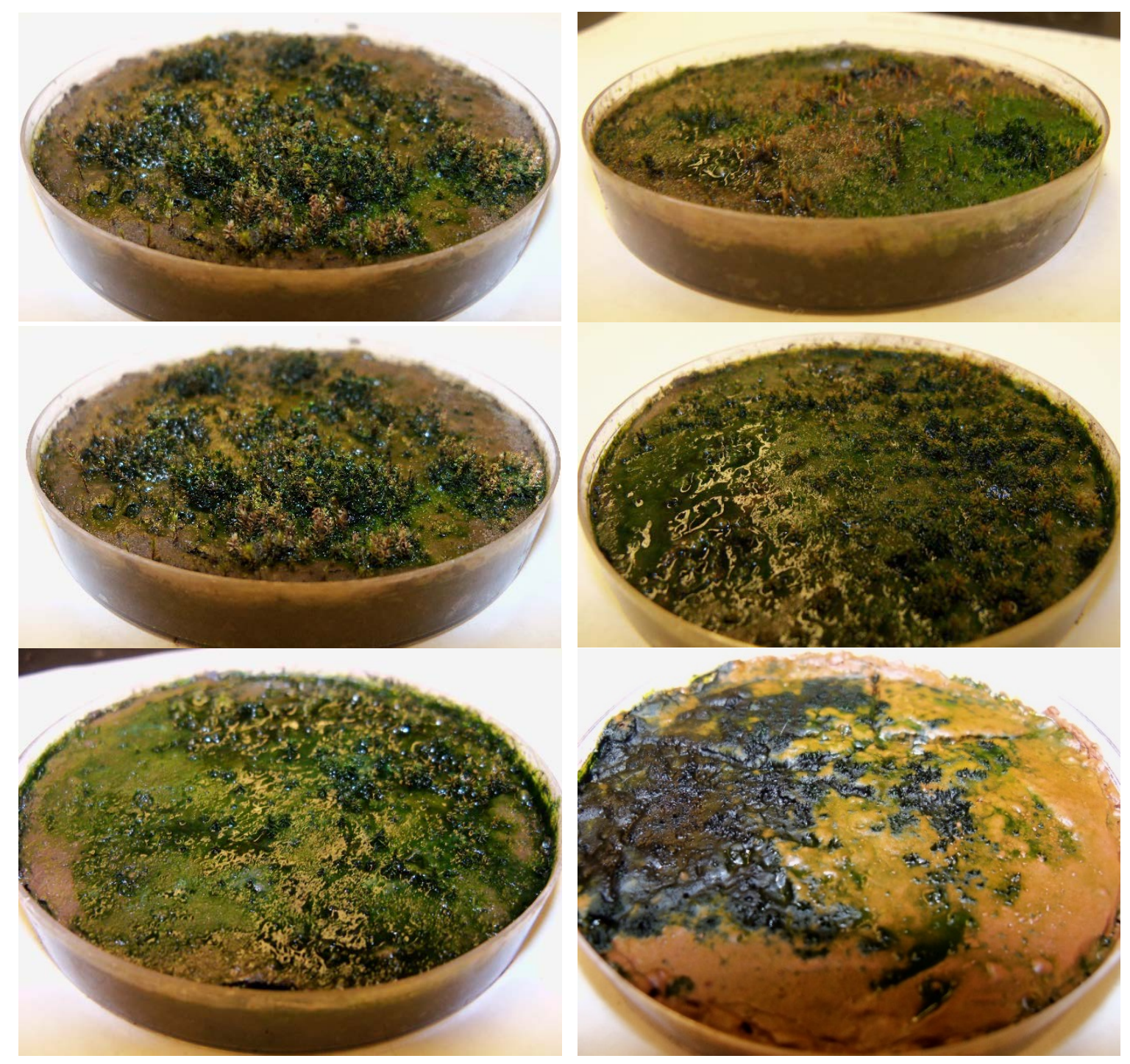

Figure 6-1 | BSCs growing on MI-mag (left) and MI-hem (right) tailings surface.

\subsection{BSC Organism Selection}

After demonstrating that BSCs could successfully grow on iron mine tailings, additional analyses were conducted to select one or more cultures suitable for use as a pioneer organism in further experimentation. Due to the many benefits of having a BSC with filamentous cyanobacteria as the pioneer organism, the native crusts that grew successfully on the mine tailings and that appeared to be cyanobacteria based on a visual assessment (e.g., black color, flat appearance) and preliminary microscopic evaluation 
were selected for further study. Specifically, three putative cyanobacterial crust samples were studied further by: (1) transferring the cultures to liquid culture flasks containing aqueous BG-11 growth medium; (2) transferring the cultures to new petri dishes containing MI-hem or MI-mag tailings (where growth on tailings plates took 5-6 weeks to appear, and 8-9 weeks to develop); and (3) observing the cultures under a light microscope at 10x and 40x magnification. All three native crust samples that were evaluated demonstrated growth in liquid medium and on iron tailings samples, and appeared to be primarily filamentous cyanobacteria when studied under a light microscope. However, based on the rapid rate of growth and almost complete coverage of the tailings plate, and the interaction between filaments observed under the microscope, (Figure 6-2), one of the native samples, referred to here as the Eagle River Crust, was selected as the native organism that would be used for further study.

The Eagle River crust has two key attributes that made it beneficial for used in this study. First, because it was isolated from the local area, it can survive the harsh winters that the MI-mag, MI-hem, and MN-mag impoundments can experience. Secondly, it has proven to grow quite successfully on tailings from these impoundments. However, the organism(s) that comprise the culture are unknown, and filamentous cyanobacteria are not the only organisms present.

To serve as a source of comparison for the performance of the native Eagle River crust, a pure culture of Anabaena doliolum, a filamentous cyanobacteria isolated from a rice field in India that had been shown to produce EPS (De Philippis et al. 2001), was obtained from the American Type Culture Collection (ATCC 43530). The freeze-dried culture was re-constituted using the recommended BG-11 medium and ATCC protocol, and later tested in the same way as described for the Eagle River culture. Although this organism grew well in liquid BG-11 and on BG-11 agar slants, it did not grow well on the iron mine tailings. These results are not discussed further in this paper, but they demonstrate the important point that not all BSC organisms are suitable for crust development on tailings. 

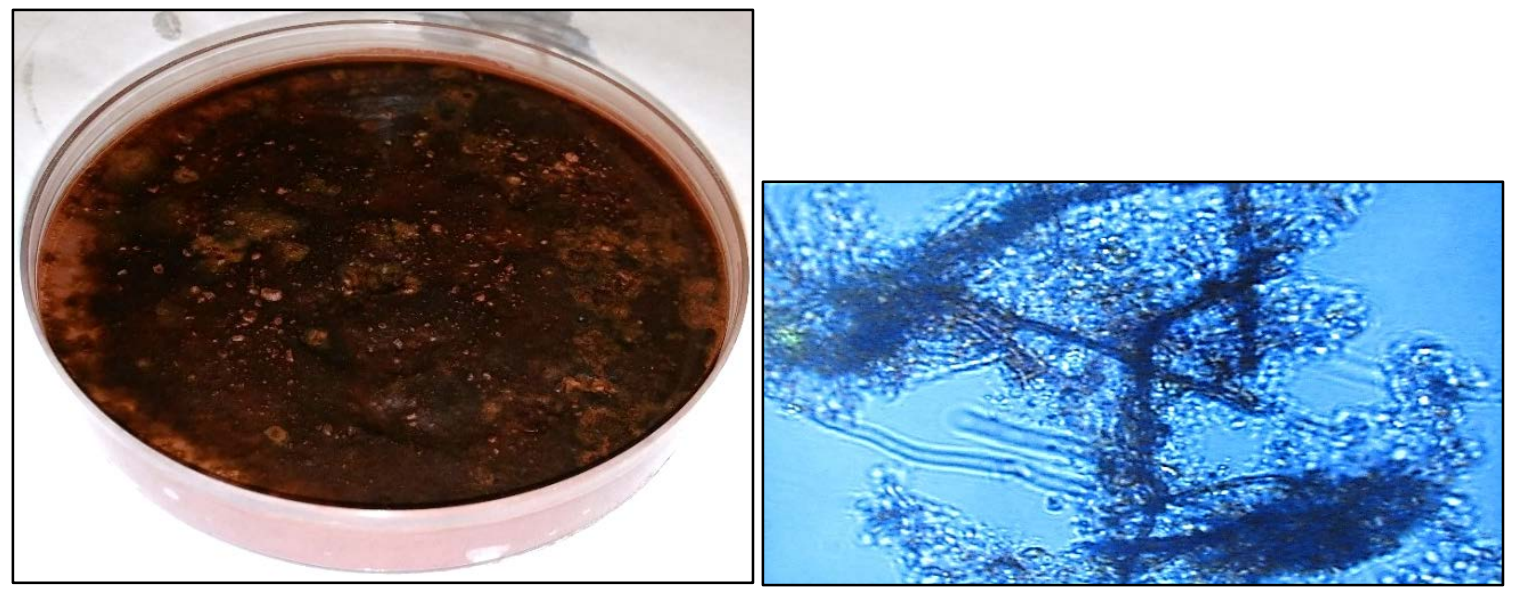

Figure 6-2 | Eagle River crust growing on MI-hem tailings (left) and viewed under light microscope at 40x magnification (right). It should be noted that the MI-hem tailings are naturally red, and the crust is black, showing almost total coverage of tailings.

\subsection{Methods for Bioreactor Inoculation, Treatment, Monitoring, Biomass Characterization, and Strength Increase Methods}

For three sets of six Eagle River (ER) bioreactors, plus one set of six BG-11 controls, the same steps were followed for inoculation, monitoring and treatment, and testing. Those methods are summarized below.

\subsubsection{Bioreactor Inoculation}

Growing and maintaining the BSCs cultures in liquid BG-11 medium proved to be effective for the Eagle River crust culture during proof-of-concept testing. Thus, liquid cultures were used to inoculate the bioreactors. Four sets of bioreactors were successfully inoculated: three sets inoculated with the Eagle River organisms that were started at different times, and a control set with no inoculum. The first set of Eagle River bioreactors was called Eagle River 1 (ER1); the second set of successful Eagle River bioreactors was called Eagle River 3 (ER3), because it followed an unsuccessful set of bioreactors (ER2) that became unhealthy and were discontinued; and the third set was called Eagle River 4 (ER4). The final, control set was called BG-11 control. 
The general procedure used for bioreactor inoculation was as follows: (1) the inoculum was grown in liquid culture, (2) the soil box bioreactors were packed with tailings, and (3) the bioreactors were inoculated. Specific details about each step follow, and details specific to each set of bioreactors are presented in Table 6-1.

To grow the inoculum, Erlenmeyer flasks containing sterile BG-11 at 20\% of their total volume were inoculated with at least $2.5 \mathrm{~mL}$ of the Eagle River sample from a previous flask, and the flask was closed with a sterile cotton plug. The flasks were subsequently incubated at room temperature in a shaking water bath under full-spectrum lights (14 hours of light/10 hours of dark) at a brightness of approximately 1000 lux. The cultures were monitored visually, and digital photos of the flasks were taken weekly and used to track culture growth in the flasks.

The soil boxes used as bioreactors were prepared following the procedures described by Buikema et al. (accepted) and Zwissler et al. (in preparation), and summarized here. All bioreactors were $75 \mathrm{~mm}$ x $75 \mathrm{~mm}$ x $75 \mathrm{~mm}$ polycarbonate boxes packed with MImag/MN-mag blend tailings to a void ratio of 0.9 , and saturated with autoclaved, distilled water. After saturation and immediately preceding inoculation, the Mariotte Tubes were disconnected from the bioreactors so that they could drain freely and the inoculum could percolate through the tailings. Also before inoculation, a $1 \mathrm{~cm}$ high polycarbonate collar was attached to the top of each soil box using Gorilla Tape and silicone caulk to provide headspace for containing the inoculum until it could permeate through the tailings. After treatment, the collar was removed by cutting through the tape with a razor blade, leaving the soil surface flush with the top of the soil box.

Once the inoculum was grown and the soil boxes were prepared and saturated, the bioreactors were inoculated, as shown in the schematic in Figure 6-3. For each set of Eagle River bioreactors, the contents of the inoculum flask(s) were poured into an autoclaved blender, and sterile BG-11 medium was added if needed, as described in Table 6-1. If BG-11 was added, it was to either dilute the inoculum to increase the volume applied to each soil box to ensure total bioreactor coverage, or to visibly match the biomass density of the previous bioreactors, as verified by comparing samples to 
photographs of previous inocula and measuring the absorbance in a spectrophotometer at three critical wavelengths $(660,700$, and $860 \mathrm{~nm})$; these wavelengths were selected due to their relevance to chlorophyll and biomass monitoring (Thenkabail et al. 2011). The contents were covered and blended at a medium to high speed setting for 2 minutes, which was selected to allow for thorough mixing while minimizing damage to the organisms (Lindahl and Bakken 1995). A $10 \mathrm{~mL}$ pipette was used to transfer $20 \mathrm{~mL}$ of inoculum from center of liquid column to each soil box. Care was used when pipetting the inoculum onto the soil surface so as to not cause unnecessary surface erosion from the stream of liquid hitting the tailings. After $20 \mathrm{~mL}$ of inoculum was distributed to each bioreactor, the blender was run on medium to high speed for another 10 seconds to resuspend the particles, and the process of distributing inoculum via pipette and resuspending particles in the blender was repeated until all inoculum was used or the desired volume of inoculum was distributed to each soil box. For the control set of bioreactors, no blending was required, and a total of $33 \mathrm{~mL}$ of sterile BG-11 was added to the top of each bioreactor via pipette and allowed to permeate through the sample in the same was the inoculum did for the treated samples. While the inoculum or growth medium was percolating through the bioreactors, they were covered with a square piece of sterile gauze to minimize contamination from airborne microbes. 
Table 6-1 | Summary of inoculum for ER1, ER3, and ER4 bioreactors, including absorbance used to compare inoculum biomass density

\begin{tabular}{lccccccc}
\hline Inoculum & $\begin{array}{c}\text { Vol. } \\
\text { from } \\
\text { flask(s) } \\
(\mathrm{mL})\end{array}$ & $\begin{array}{c}\text { Vol. } \\
\text { after } \\
\text { dilution } \\
(\mathrm{mL})\end{array}$ & $\begin{array}{c}\text { Reason for } \\
\text { inoculum dilution }\end{array}$ & $\begin{array}{c}\text { Vol. of } \\
\text { inoculum } \\
\text { per box } \\
(\mathrm{mL})\end{array}$ & $\mathrm{A}_{660}$ & $\mathrm{~A}_{700}$ & $\mathrm{~A}_{860}$ \\
\hline ER1* & 400 & 400 & $\mathrm{n} / \mathrm{a}$ & 66 & - & - & - \\
\hline ER3 & 100 & 200 & $\begin{array}{l}\text { Matching biomass } \\
\text { density of ER1 }\end{array}$ & 33 & 1.361 & 1.158 & 0.821 \\
\hline ER4 & 100 & 240 & $\begin{array}{l}\text { Increase volume of } \\
\text { inoculum }\end{array}$ & 40 & 0.749 & 0.630 & 0.423 \\
\hline $\begin{array}{l}\text { ER4- } \\
\text { reinoc** }\end{array}$ & 100 & 300 & $\begin{array}{l}\text { Increase volume of } \\
\text { inoculum }\end{array}$ \\
* The biomass of ER1 was not quantitatively measured, but was rather documented in \\
photographs showing the color of the inoculum. ER3 inoculum was diluted to visually \\
match the color of the ER1 inoculum, and then biomass was roughly characterized using \\
absorbance. \\
** Due to the low volume and low biomass applied to ER4 bioreactors, crust \\
development was slow, and to increase the speed the bioreactors were re-inoculated after \\
$\begin{array}{l}\text { 21 days, which increased the rate of crust development significantly in the final 10 days } \\
\text { of bioreactor maintenance. }\end{array}$
\end{tabular}

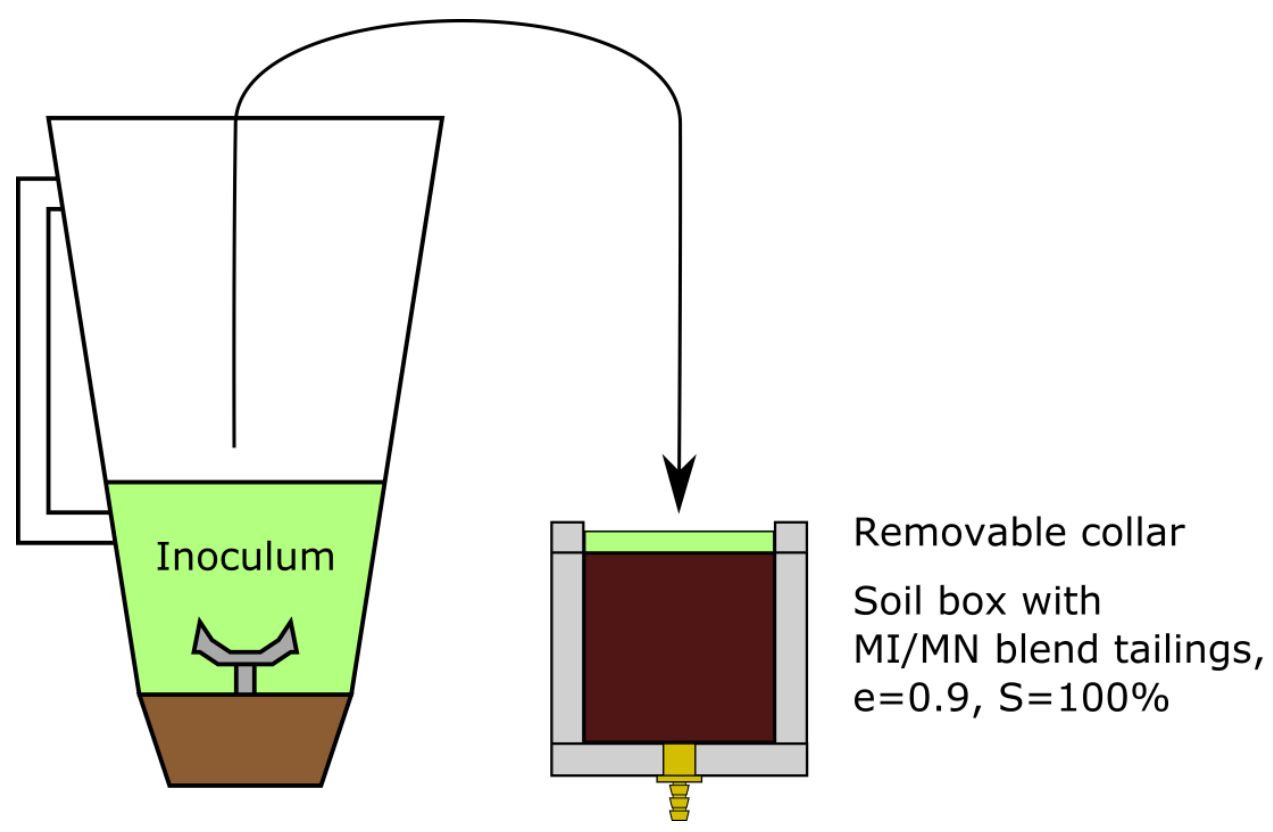

Figure 6-3 | Schematic of bioreactor inoculation, where one batch of $400 \mathrm{~mL}$ inoculum was divided evenly between 6 soil boxes, featuring removable collars to provide extra headspace in which the inoculum could reside until percolating through the tailings sample. 


\subsubsection{Bioreactor Treatment and Monitoring}

After preparation, inoculated and control bioreactors were maintained and monitored. All bioreactors were stored in clear plastic tubs, with lids loosely placed on top to allow air circulation while minimizing contamination by airborne microorganisms. The bioreactors were incubated at room temperature under 1600 to 1800 lux of full-spectrum light on a 14 hour light/10 hour dark cycle, as recommended by ATCC for A. doliolum. The bioreactors were monitored and maintained two times per week. On each occasion, the samples were monitored using remote sensing. Digital photographs were taken of the sample surface to qualitatively monitor crust growth and development. In addition, for quantitative monitoring, an ASD FieldSpec ${ }^{\circledR}$ HandHeld Pro Spectroradiometer (325$1075 \mathrm{~nm}$ ) with plant probe was used to collect multispectral reflectance data for the crusts. For each sample, the average of 10 spectral reflectance curves collected at two different locations on the sample surface ( 5 curves per location) was used to monitor biomass with vegetation indices, including the Normalized Difference Vegetation Index, or NDVI (Thenkabail et al. 2011). To avoid possible cross-contamination, the plant probe surface was disinfected with ethanol between each sample. After completing the monitoring, the samples were maintained with food/water. Once per week, the samples were fed with BG-11 medium, and a second time per week, the samples were moistened with sterile distilled water. In each case, the fluid was applied using a squirt bottle, taking care not to cause surface erosion. Sufficient volume was applied to keep the sample moist, but not saturated.

The bioreactors were monitored and maintained until crusts were sufficiently developed, at which time the biomass of the crust was quantified and the BSC resistance to freezing/sublimation and wind erosion was tested, as described in the following sections. For each set of six bioreactors, one was used for biomass characterization and the remaining five were used to study freezing/sublimation and wind erosion resistance. 


\subsubsection{Biomass Characterization}

To characterize the biomass present in each set of BSCs being studied for wind erosion resistance, one bioreactor sample per set of six was sacrificed with destructive biomass testing. Samples of each crust were collected using syringe barrel soil coring and were oven dried. These dry samples were then used for additional analysis, including polysaccharide content via phenol-sulfuric acid reaction assay and chlorophyll a content using spectrophotometry and a trichromatic equation.

The first step in biomass characterization was obtaining small sub-samples of the bioreactor, each containing the same amount of crust and underlying tailings for repeatability in analyses. This was accomplished with syringe barrel coring. A small syringe (10 mL BD syringe with Luer-Lok tip, cat 309604) with an inner diameter of 1.4 $\mathrm{cm}$ had its tip cut off and was used for coring. Given the dimensions of the syringe and soil box, up to 16 cores could be extracted. The syringe corer was inserted into the bioreactor to a depth of $0.65 \mathrm{~cm}$, with the syringe plunger in the $3 \mathrm{~mL}$ position. Next, the syringe plunger was pulled from the $3 \mathrm{~mL}$ to $6 \mathrm{~mL}$ mark (a distance of $1.95 \mathrm{~cm}$ ) to create suction, and then the syringe containing a core of crust and tailings was removed. The plunger was pushed through the syringe to extract the core, which was trimmed using a razor to a sample height of 0.1 " or 0.2 ", and placed into a sterile ceramic weighting dish. The cores were then dried at $110^{\circ} \mathrm{C}$ for 24 hours, and the dry weight was measured. This procedure, which was proven in previous tests to be repeatable, yielded samples that had a dry weight between 0.9 and 1.3 grams for the 0.1 " height cores and between 1.5 and 2.25 grams for the 0.2 " cores. These sample cores were used for all other analyses.

The polysaccharide content of the BSC samples, used to quantify EPS produced by the filamentous cyanobacteria, was measured with the phenol-sulfuric acid reaction assay, as described by Dubois et al. (1956). To perform the phenol-sulfuric acid reaction, polysaccharides were separated from soil particles using methods presented by Colica et al. (2015). For each set of BSC samples, at least two 0.1" cores were used for measurement of polysaccharides in each sample. From each core, 1 gram of dry sample was ground using a sterile mortar and pestle and placed into a $15 \mathrm{~mL}$ centrifuge tube 
(VWR $15 \mathrm{~mL}$ polypropylene centrifuge tube with conical-bottom, cat 89039). To each tube, $15 \mathrm{~mL}$ of $0.1 \mathrm{M} \mathrm{Na} 2$ EDTA was added, and the tube was mixed and then allowed to sit for 15 minutes at $25^{\circ} \mathrm{C}$. The tubes were centrifuged for 20 minutes at $3500 \mathrm{x} \mathrm{g}(5440$ $\mathrm{rpm}$ ) to recover the polysaccharide extracts devoid of any soil particles in suspension. The supernatant was removed from the centrifuge tube and saved for analysis, while the cell/soil pellet was rehydrated with $15 \mathrm{~mL}$ of fresh $0.1 \mathrm{M} \mathrm{Na}{ }_{2}$ EDTA, allowed to sit for 15 minutes at $25^{\circ} \mathrm{C}$, and then centrifuged again for 20 minutes at $3500 \mathrm{x}$ g. This process was repeated for a total of at least 4 extractions per tube. The supernatants from each tube and each extraction were stored at $4^{\circ} \mathrm{C}$ before analysis. The phenol-sulfuric acid reaction was then carried out on all supernatants from each core, in duplicate. First, test tubes were filled with $0.5 \mathrm{~mL}$ of supernatant, or where dilutions were necessary to get absorbance readings within the range of the standard curve prepared, $0.25 \mathrm{~mL}$ of supernatant and $0.25 \mathrm{~mL}$ of distilled water was used. For the calibration curve, a blank was filled with 0.5 $\mathrm{mL}$ of distilled water, and glucose standards were filled with $0.5 \mathrm{~mL}$ of solutions containing 10 to $100 \mu \mathrm{g} / \mathrm{mL}$ of glucose. Next, $0.5 \mathrm{~mL}$ of phenol reagent $(80 \%$ phenol solution by weight) was added to all tubes and mixed rapidly and thoroughly. Subsequently, $2.5 \mathrm{~mL}$ of reagent grade sulfuric acid was added to all tubes and mixed rapidly. The tubes were left to stand for 10 minutes, and then placed in a $25^{\circ} \mathrm{C}$ water bath for 15 minutes. The contents of each tube was transferred to a cuvette, and the absorbance measured at $488 \mathrm{~nm}\left(\mathrm{~A}_{488}\right)$ against the distilled water blank. The glucose standard curve, which was developed using at least three readings collected at each of six glucose concentration over the span of three months, was verified every time the assay was performed using a standard of $100 \mu \mathrm{g} / \mathrm{mL}$ of glucose. This standard curve was used to determine polysaccharide concentration for each tube, and the polysaccharide concentration for each extraction was added to determine the total polysaccharide concentration for the sample. The concentration of polysaccharides measured in each sample, with quadruplicate results for each biological soil crust sample, served as a means of comparison between the biomass of the different biological soil crusts. 
Chlorophyll a content was measured using a spectrophotometer and trichromatic equations, based primarily on the methods of Liu et al. (2008). For each set of biological soil crust samples, two 0.2 " cores were used for chlorophyll a determination. For each core, 2 grams of dry sample was ground using a sterile mortar and pestle and placed into a $15 \mathrm{~mL}$ centrifuge tube (VWR $15 \mathrm{~mL}$ polypropylene centrifuge tube with conicalbottom, cat 89039 ). To this tube, a $90 \%$ acetone solution was added to reach a total volume of $10 \mathrm{~mL}$, and then $2.5 \mathrm{~mL}$ of DMSO was added. The tubes were refrigerated overnight in the dark at $4{ }^{\circ} \mathrm{C}$, and then were centrifuged at $7000 \mathrm{rpm}$ for 10 minutes. The supernatant was separated, and analysis of each supernatant was done in duplicate. For each duplicate sample, $3 \mathrm{~mL}$ of supernatant was added to a cuvette, or, where dilutions were necessary to get absorbance readings within the range of the standard curve prepared, $1.5 \mathrm{~mL}$ of supernatant and $1.5 \mathrm{~mL}$ of $90 \%$ acetone was used. For each cuvette, the absorbance was measured at $384 \mathrm{~nm}, 490 \mathrm{~nm}$, and $663 \mathrm{~nm}$ against a $90 \%$ acetone blank using a Thermo Scientific Genesys 20 spectrophotometer. The trichromatic equations presented by Garcia-Pichel and Castenholz (1991) were then used to calculate the chlorophyll a concentration in units of absorbance/sample weight. The trichromatic equations used correct chlorophyll a absorbance for interferences caused by carotenoids and scytonemin. The potential interference of turbidity on chlorophyll a absorption was investigated in this study by measuring the absorbance of the samples at $750 \mathrm{~nm}$, and the effect of pheophytin a on chlorophyll a absorption was investigated by measuring the absorbance of the samples at $664 \mathrm{~nm}$ before acidification and $665 \mathrm{~nm} 90$ seconds after acidification with $0.1 \mathrm{~mL}$ of $0.1 \mathrm{~N} \mathrm{HCl}$ (American Public Health Association 1994). Based on these analyses, the effects of turbidity and pheophytin were negligible for the Eagle River crusts. To convert to units of $\mu \mathrm{g}$ chl a/g sample, a standard chlorophyll a curve was developed by mixing 6 dilutions of a standard chlorophyll a solution, measuring the absorbance of the dilutions at $384 \mathrm{~nm}, 490 \mathrm{~nm}$, and $663 \mathrm{~nm}$, applying the trichromatic equations, and plotting against the known concentration of chlorophyll a in each dilution; the resulting chlorophyll a standard curve was only developed once. During the entire chlorophyll a extraction and measurement procedure, care was taken to keep samples cold $\left(4^{\circ} \mathrm{C}\right)$ and in the dark, as exposure to warm temperatures and light can 
cause degradation of extracted chlorophyll. The amount of chlorophyll a in each sample, determined with quadruplicate results through the use of the trichromatic equations and the chlorophyll a standard curve, served as an additional means of comparison between the biomass of the different biological soil crusts.

\subsubsection{Scanning Electron Microscopy}

Environmental scanning electron microscopy, or ESEM, was used to analyze the structure of the BSCs and the interaction between the filamentous cyanobacteria and the tailings particles. For the ER1 crust, which was assumed to be representative of the structure of all crusts originating from the same inoculum, a small piece of crust was obtained from the remaining crust surrounding the extracted cores, and was removed aseptically with microforceps and placed in a crucible. The crust was dried at $40^{\circ} \mathrm{C}$ for 3 days, and the dried crust was used to make the SEM mount. The crust was affixed to the sample mount using carbon tape and graphite paste, coated with 10 micron carbon coating (Buikema 2015), and then observed using ESEM.

\subsubsection{Bioreactor Freezing and Sublimation}

For each set of bioreactors, the remaining 5 bioreactors that were not destructively sampled for biomass characterization were used to study crust resistance to sublimation and wind erosion. The laboratory equipment and methods used are described by Zwissler et al. (in preparation), and summarized briefly here.

Samples were exposed to one-dimensional freezing from the surface down, which is representative of how the ground freezes. This was accomplished using a freezer, heating/refrigerated recirculating chiller attached to a water bath, and insulation. Up to 6 samples were tested in the freezer at one time. Each bioreactor was connected to a distilled water source using an insulated Mariotte tube line, and was loaded into a water bath within the freezer. Sufficient antifreeze was added to the water bath to fill it within $1-2 \mathrm{~cm}$ of the bottom of the bioreactors, thereby keeping the bottom of the sample and Mariotte tube lines from freezing. The water bath was attached to the heating/refrigerated recirculating chiller, which maintained an antifreeze temperature of $+5^{\circ} \mathrm{C}$. Once all soil 
boxes were in place, the voids between the soil boxes and the edge of the water bath were filled with Styrofoam insulation to prevent freezing of the samples from the sides. The freezer was then set to $-5^{\circ} \mathrm{C}$ for seven days, which was sufficient time to freeze the samples, generate ice lenses, and cause sublimation to occur.

\subsubsection{Wind Tunnel Testing}

At the conclusion of the 7 day freezing/sublimation cycle, the bioreactors were used to assess wind erosion resistance. One way this was done was with wind tunnel testing. These methods are described in detail by Zwissler et al. (in preparation), and summarized briefly here.

For the wind tunnel testing, sample mass lost was the primary tool used to quantitatively compare wind erosion for the different crust types and untreated tailings. Digital photographs of the samples before and after wind tunnel testing, and video recordings of the wind tunnel tests, were also used for qualitative analysis of the crust behavior in the wind tunnel. Samples removed from the freezer were tested in the wind tunnel by being placed in the sample hole in the base of the wind tunnel, with the sample supported by a lab jack. The sample was surrounded by a plywood plug to fill any voids between the sample box and the edge of the wind tunnel base, with the sample surface level and flush with the wind tunnel base. Then, the samples were exposed to 30 seconds in the wind tunnel at $96.6 \mathrm{~km} / \mathrm{hr}(60 \mathrm{mph})$. This exposure time was selected to minimize sample melting which confounded the experimental results. After testing, the sample was carefully removed from the wind tunnel so as to not cause sample disturbance and additional mass lost due to transport, and the sample mass was measured to quantify mass lost in the wind tunnel. Digital photographs were also taken to document any visible change to the sample incurred during wind tunnel testing.

Importantly, not all bioreactors were tested with the wind tunnel. It was discovered during testing of the ER1 bioreactors that, if the leading edge of the crust was curled up or had any voids below it (e.g., from sublimated ice lenses), the wind tunnel could remove the entire crust suddenly, making further testing impossible and yielding the 
sample useless. Due to the small sample surface area, the edges were not sealed to avoid this problem as has been done in other studies (e.g., McKenna Neuman et al. 1996), due to concerns that it might falsely exaggerate the crust strength. Therefore, for the ER3 and ER4 samples, if there was a concern about the crust potentially flaking off in the wind tunnel due to detachment along the edges, it was not tested.

\subsubsection{Ball Drop Testing}

For the bioreactors that were not tested in the wind tunnel due to concerns about the crust flaking off, and for bioreactors that did not see notable surface disturbance due to wind tunnel testing, an alternative erosion resistance test was used. This measure of the surface crust strength, called the steel bearing drop test or ball drop test, was developed and described in detail by Buikema et al. (accepted) and Zwissler et al. (in preparation), and is briefly described here.

For each sample tested, a $3.4 \mathrm{~g}$ steel ball bearing with a diameter of $0.945 \mathrm{~cm}$ was dropped onto the soil surface from a height of $1.5 \mathrm{~m}$. The ball bearing was subsequently removed with a magnet, the impact crater left on the soil surface was measured in the $\mathrm{x}$ and y axis using a digital caliper, and the two crater diameter values were averaged. This test was repeated 5 times for each sample, with one impact crater in each quadrant of the sample surface, and one impact crater in the center of the sample surface. The impact crater diameter was the primary means of quantitative comparison between the resistance to impact provided by the different crust types and untreated tailings.

\subsection{Results and Discussion}

\subsubsection{Bioreactor Inoculation}

Eagle River bioreactors were successfully inoculated as detailed in Table 6-1. The Eagle River inoculum, as observed under a light microscope under 40x magnification, is shown in Figure 6-4. As expected based on the initial microscopic analysis of the Eagle River sample (Figure 6-2), the inoculum featured both unicellular organisms and, more importantly, filaments presumed to belong to filamentous cyanobacteria. Filaments of 
various lengths can be seen in these images, which demonstrates how development occurs.
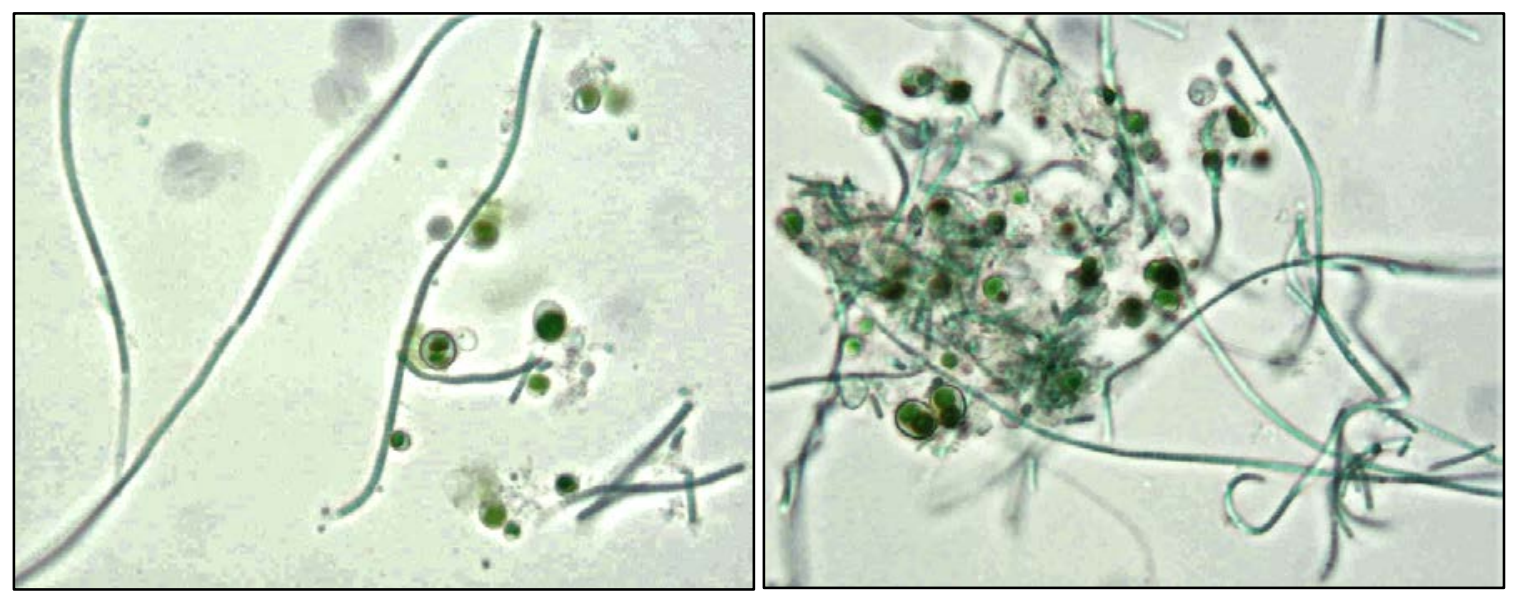

Figure 6-4 | Eagle River inoculum viewed under light microscope at 40x magnification, showing the presence of both filaments and unicellular organisms.

\subsubsection{Bioreactor Treatment and Monitoring}

Digital photography images used for qualitative monitoring of each set of ER bioreactors at different phases of treatment are shown in Figure 6-5 (ER1), Figure 6-6 (ER 3), and Figure 6-7 (ER4). For the ER bioreactors, while the image at the start of inoculation might show some green color indicative of the presence of the inoculum, the development of the crust with time is visually quite evident. Such visual monitoring is useful for identifying that a crust is developing, but it does not provide any way to quantitatively assess the state of crust development. Therefore, NDVI was used for quantitative monitoring of the crust development, crust coverage, and crust health during treatment. The trends in NDVI throughout the treatment phase for all ER and BG-11 control bioreactors are shown in Figure 6-8.

For ER1, all bioreactors remained healthy during the duration of the treatment phase, so all bioreactors exhibited the same behavior and had similar NDVI trends throughout (Figure 6-8a). Rapid crust development took approximately 35 days, as indicated by the steep increase in NDVI, after which the bioreactors were completely covered with healthy biomass and changes to NDVI were minor. 
Similarly, for ER3, three of the bioreactors exhibited good health, exhibited similar behavior, and had similar NDVI trends throughout the treatment phase (Figure 6-8b). For these three bioreactors, rapid crust development took approximately 20 days, as indicated by the steep increase in NDVI, after which the bioreactors were completely covered with healthy biomass and changes to NDVI were minor. However, a fourth ER3 bioreactor shared the same 20 day crust development phase, but showed some signs of distress after the crust was developed, as indicated by the decrease in NDVI before rebounding to a NDVI similar to the three healthy bioreactors as health improved. The remaining two bioreactors began to show signs of distress as early as 15 days post-inoculation, and while the source of the distress could not be identified, it is clear that the health of these remaining two bioreactors never recovered, and, thus, they were not used for testing.

In comparison, for ER4, both visual (Figure 6-7) and NDVI monitoring (Figure 6-8c) showed that the low volume of lower biomass-density inoculum (Table 6-1) was insufficient to cause rapid crust development on the tailings within the first 21 days of treatment. In an effort to increase the rate of crust development, the bioreactors were reinoculated on day 21 , which caused a small but immediate increase in NDVI, indicating crust development, with one bioreactor reaching higher NDVI values during the final 10 days of the treatment phase.

For the BG-11 control bioreactors, no change in NDVI was observed throughout the treatment phase (Figure 6-8d), consistent with the visual assessment that no biological crust was developed. Because no inoculum was applied to these control bioreactors, the lack of biological development suggests that no native phototrophic organisms were stimulated by the addition of the BG-11 growth medium, a commonly used nutrient source for cyanobacteria. Based on this observation, any BSC to be grown on these tailings would need to be initiated with an inoculation phase. 

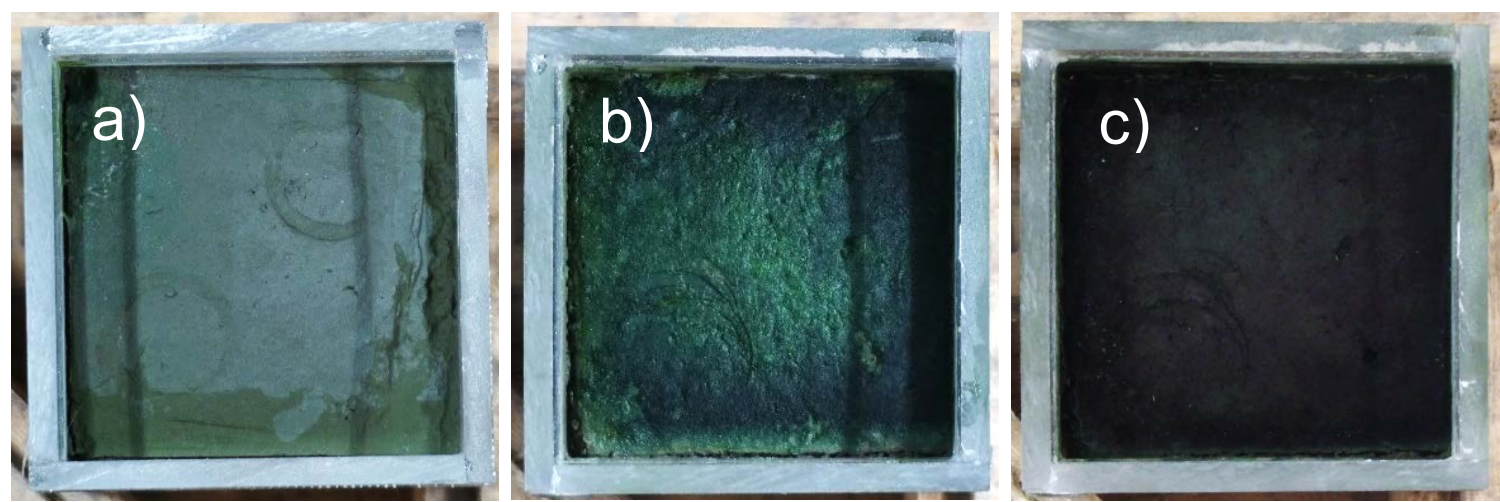

Figure 6-5 | Digital photographs of ER1 bioreactors used to qualitatively document crust development (a) at the start of treatment (day 3) with a light green color completely covering the tailings surface, (b) after full crust development (day 37) with a darker green or black color completely covering the tailings surface, and (c) at the end of treatment (day 134) with a thick and healthy black crust. Circular imprints in the crust are from the spectroradiometer probe.
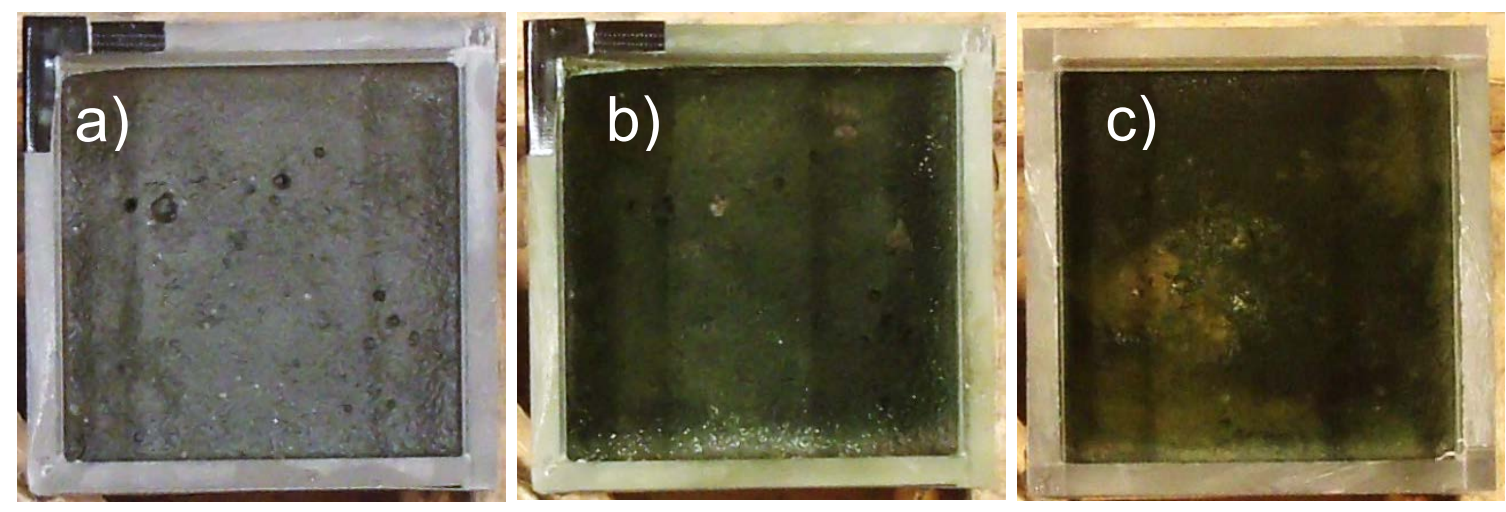

Figure 6-6 | Digital photographs of ER3 bioreactors used to qualitatively document crust development (a) at the start of treatment (day 1) with little to no coverage of the tailings surface, (b) at the end of treatment (day 42) for a healthy bioreactor with a darker green crust completely covering the tailings surface, and (c) at the end of treatment (day 42) for an unhealthy bioreactor with patchy crust coverage of the tailings surface. 

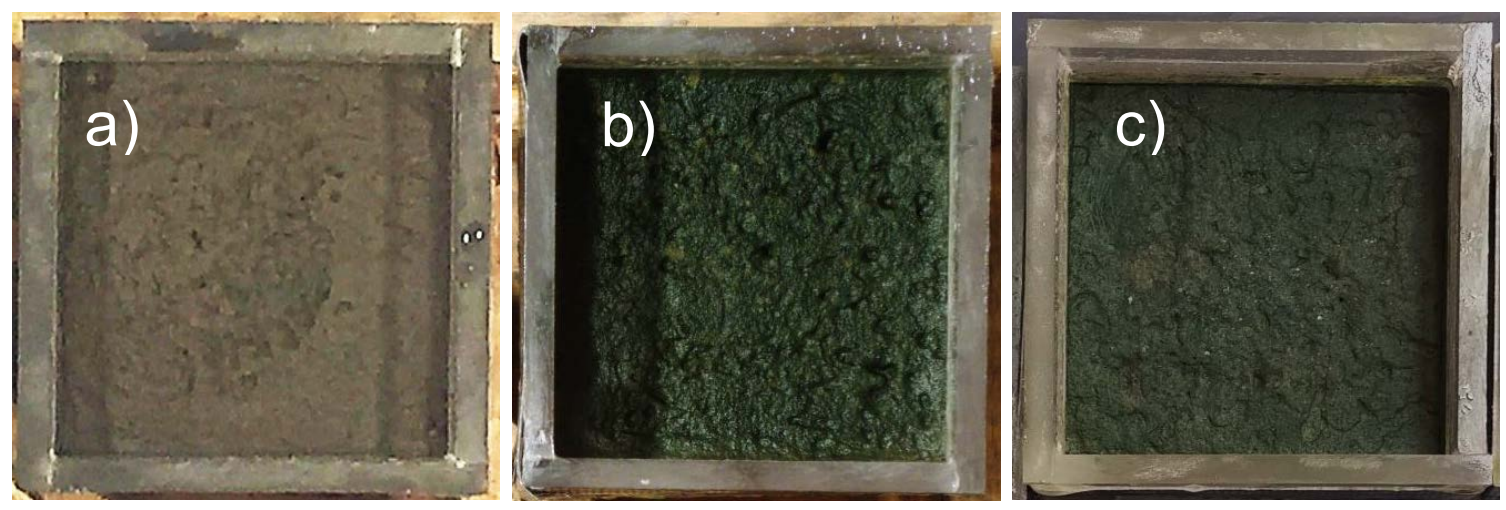

Figure 6-7 | Digital photographs of ER4 bioreactors used to qualitatively document crust development (a) at the start of treatment (day 1) with little to no coverage of the tailings surface, (b) after reinoculation (day 24) with a more noticeable green color completely covering the tailings surface, and (c) at the end of treatment (day 31) with a thick and healthy dark crust. 

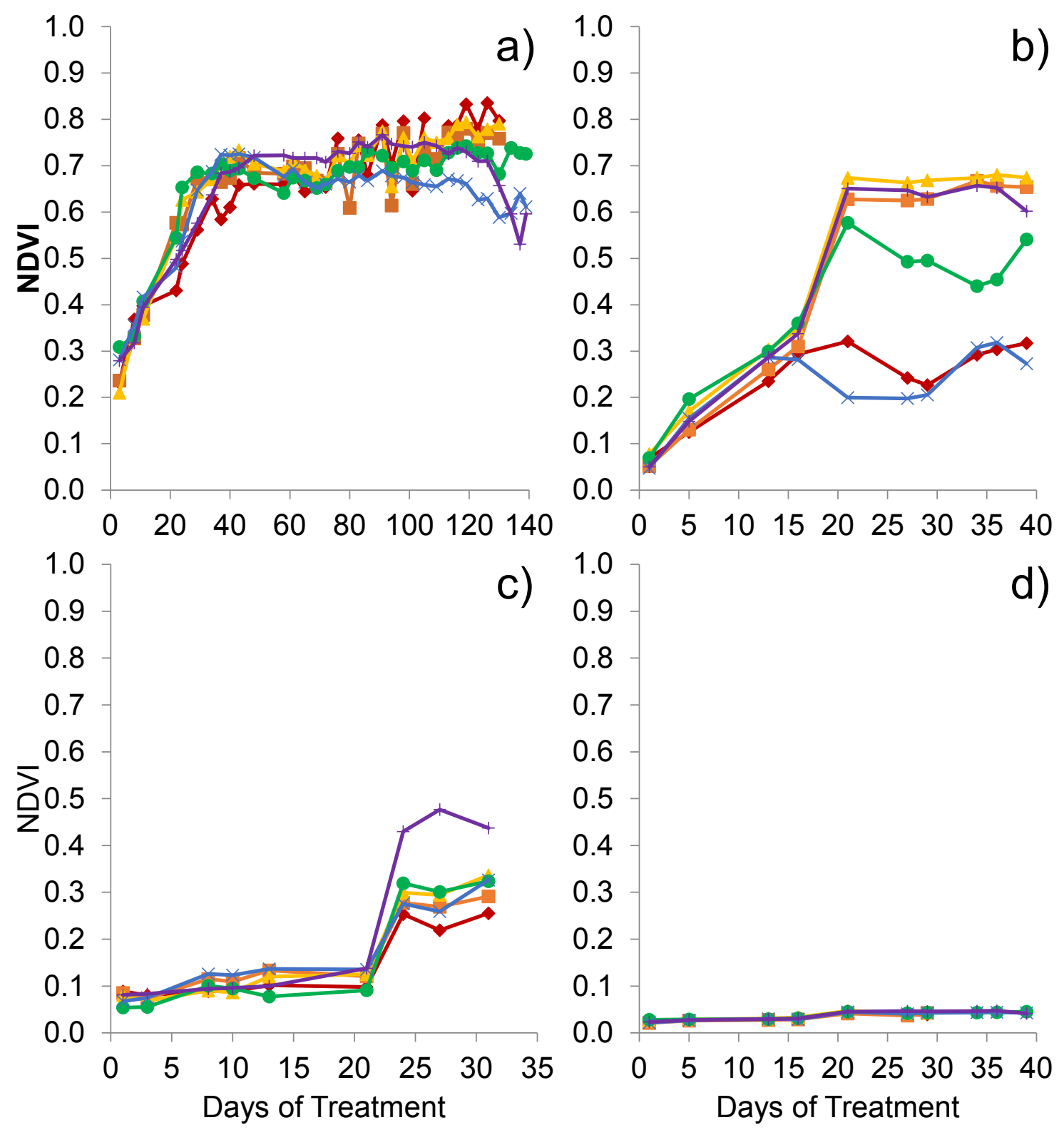

Figure 6-8 | NDVI used for quantitative crust monitoring of (a) Eagle River 1 bioreactors, (b) Eagle River 3 bioreactors, (c) Eagle River 4 bioreactors, and (d) BG11 control bioreactors, showing that crust development can be monitored using NDVI, full crust coverage can be identified when NDVI levels off, and crust health can be assessed by watching for a decrease in NDVI.

\subsubsection{Biomass Characterization}

At the conclusion of the treatment phase, one sample was sacrificed for each set of bioreactors to collect cores for biomass characterization via the chlorophyll a (chl a) and 
EPS concentration, as summarized in Table 6-2. At least 2 sample cores were used for each method, and all assays were run in duplicate. Chlorophyll a was clearly produced, as expected for the putative cyanobacteria in the ER culture. In addition, EPS was also clearly produced by the ER culture, which is important given the role of EPS in soil stabilization. Given the questions about the health of the ER3 crusts, it is not surprising that the organisms produced smaller quantities of EPS in that case.

Because the BG-11 controls were not inoculated with any organisms and did not show any signs of biomass development during treatment and monitoring, it is assumed that the chlorophyll a and EPS concentrations detected for these bioreactors (Table 6-2) represent background values associated with these methods. All ER bioreactors had concentrations well above these presumed background values, and are therefore considered to be valid.

Not surprisingly, given the trends in NDVI data with treatment time, the data in Table 6-2 also suggest trends in the biomass measures, especially chlorophyll a, with time. To examine these trends, the biomass concentrations with respect to treatment time are presented in Figure 6-9. Although the data set is limited, for the chlorophyll a and EPS results from the ER bioreactors, there was a clear trend between treatment time and biomass production. In particular, chlorophyll a, which was expected to be produced by the ER culture, had a clear exponential trend $\left(\mathrm{R}^{2}=0.93\right)$, which is consistent with exponential growth of the microorganisms. The EPS data are closely grouped for the ER bioreactors, with the exception of ER3, but can still be represented with a linear trendline $\left(\mathrm{R}^{2}=0.49\right)$. While not confirmed, it is suspected that the healthy ER crust organisms either stop or slow down production of EPS after a certain amount is produced (between 15 and $19 \mu \mathrm{g} / \mathrm{g}$ based on these data), whether limited by nutrients, space, or other factors.

To investigate if the quantitative remote sensing tool, NDVI, used to monitor crust development is effective at characterizing biomass concentration, as measured by either chlorophyll a or EPS, the chlorophyll a and EPS values measured at the conclusion of treatement were compared to final NDVI (calculated to be the average NDVI for all healthy bioreactors once peak crust development was reached), as illustrated in Figure 
6-10. Based on these data, it is clear that NDVI has a stronger correlation with chlorophyll a $\left(\mathrm{R}^{2}=0.85\right)$ than it does with EPS $\left(\mathrm{R}^{2}=0.50\right)$. This is reasonable given that NDVI is used in remote sensing to assess if the target area contains live green vegetation (Thenkabail et al. 2011). These results indicate that, with further development, NDVI could potentially be used to predict the biomass concentration of a developing phototrophic biological soil crust, using chlorophyll a as a proxy.

Table 6-2 | Summary of biomass characterization data for Eagle River Bioreactors and BG-11 Control Bioreactors

\begin{tabular}{lccccccc}
\hline \multirow{2}{*}{ Bioreactor } & \multicolumn{2}{c}{$\begin{array}{c}\text { Days of } \\
\text { Treatment }\end{array}$} & Chl a $(\mu \mathrm{g} / \mathrm{g})$ & EPS $(\mu \mathrm{g} / \mathrm{g})$ & \multicolumn{2}{c}{$\begin{array}{c}\text { Number of } \\
\text { Successful } \\
\text { (Attempted) Tests }\end{array}$} \\
\cline { 3 - 8 } & & Avg. & $\begin{array}{c}\text { Std. } \\
\text { dev. }\end{array}$ & Avg. & $\begin{array}{c}\text { Std. } \\
\text { dev. }\end{array}$ & $\begin{array}{c}\text { Wind } \\
\text { Tunnel }\end{array}$ & $\begin{array}{c}\text { Ball } \\
\text { Drop }\end{array}$ \\
\hline ER1 & 131 & 433 & 99.6 & 18.6 & 2.1 & $1(3)$ & $1(1)$ \\
ER1-2* & 140 & 734 & 52.7 & 16.9 & 0.6 & $1(1)$ & $1(1)$ \\
ER3 & 42 & 160 & 19.6 & 7.9 & 1.0 & $1(1)$ & $3(4)$ \\
ER4 & 31 & 78 & 5.2 & 15.2 & 0.6 & $2(3)$ & $4(4)$ \\
BG-11 control & 42 & 36 & 5.5 & 2.0 & 0.8 & $5(5)$ & $5(5)$ \\
\hline
\end{tabular}

*ER1 bioreactors were tested in two sets, nine days apart. Biomass was measured on both occasions in case a significant amount of biomass was generated in those nine days. 


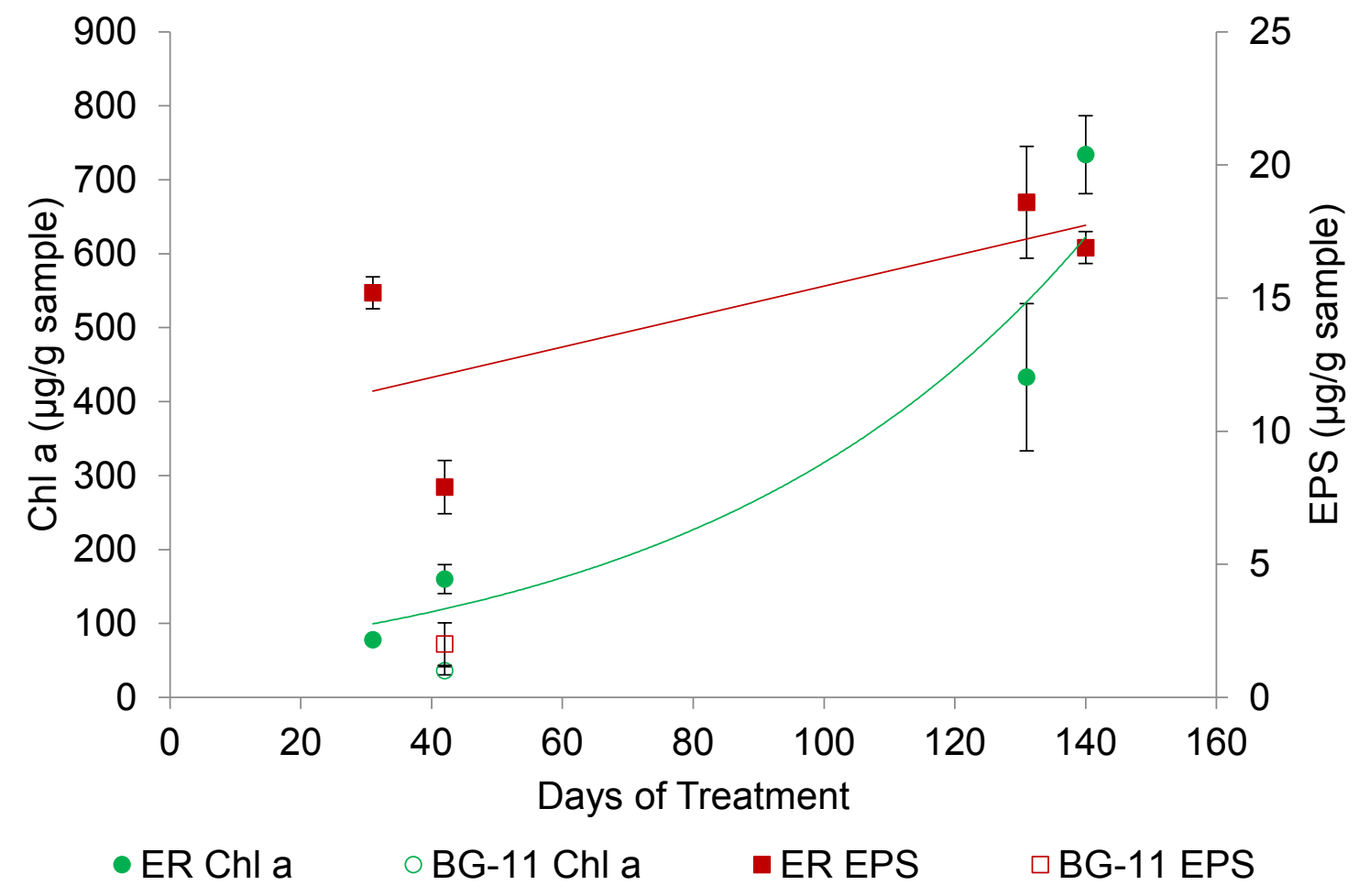

Figure 6-9 | Biomass characterization data for Eagle River bioreactors, showing that biomass concentration is a function of treatment time and that biomass of treated samples far exceeds biomass of BG-11 controls. Each point represents the average value for each bioreactor, and the error bars represent standard deviation. 


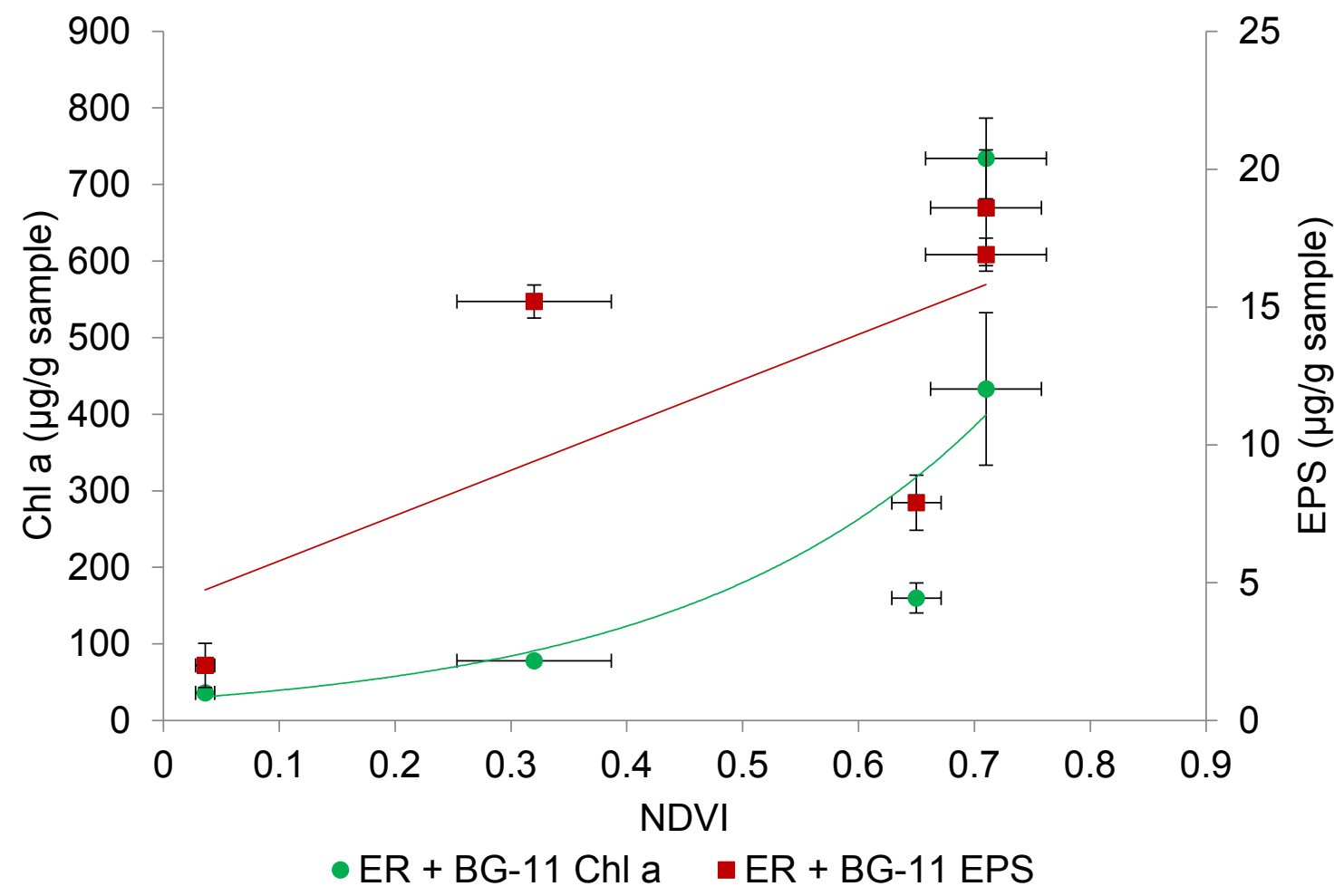

Figure 6-10 | Relationship between NDVI and biomass concentration, showing that NDVI has a stronger correlation with chl a $\left(\mathrm{R}^{2}=0.85\right)$ than it does with EPS $\left(\mathrm{R}^{2}=0.50\right)$. Each point represents the average final NDVI for each bioreactor set versus the average biomass concentration for each bioreactor, and the error bars represent standard deviation.

\subsubsection{Scanning Electron Microscopy}

ESEM images for the ER1 crust, illustrating the interaction between the filamentous cyanobacteria and the tailings particles, are presented in Figure 6-11(b-f). As a point of comparison for the biological crusts, SEM imagery for untreated magnetite tailings (Figure 6-11(a)) shows that the tailings particles are relatively smooth and angular, without any notable coatings. In comparison, the SEM images of the ER1 crust (Figure 6-11(b-f)) show a sharp contrast to the untreated tailings, including a visible coating that is presumed to be EPS. In addition to this coating, biological organisms can be seen in the images, including rod-shaped organisms and filaments clearly interacting with the tailings/crust surface. This imagery demonstrates that the crust is not only growing on top of the tailings, but that the Eagle River organisms are able to interact with the tailings 
through the presence of EPS and filaments, presumably forming a stronger and more resilient crust. 


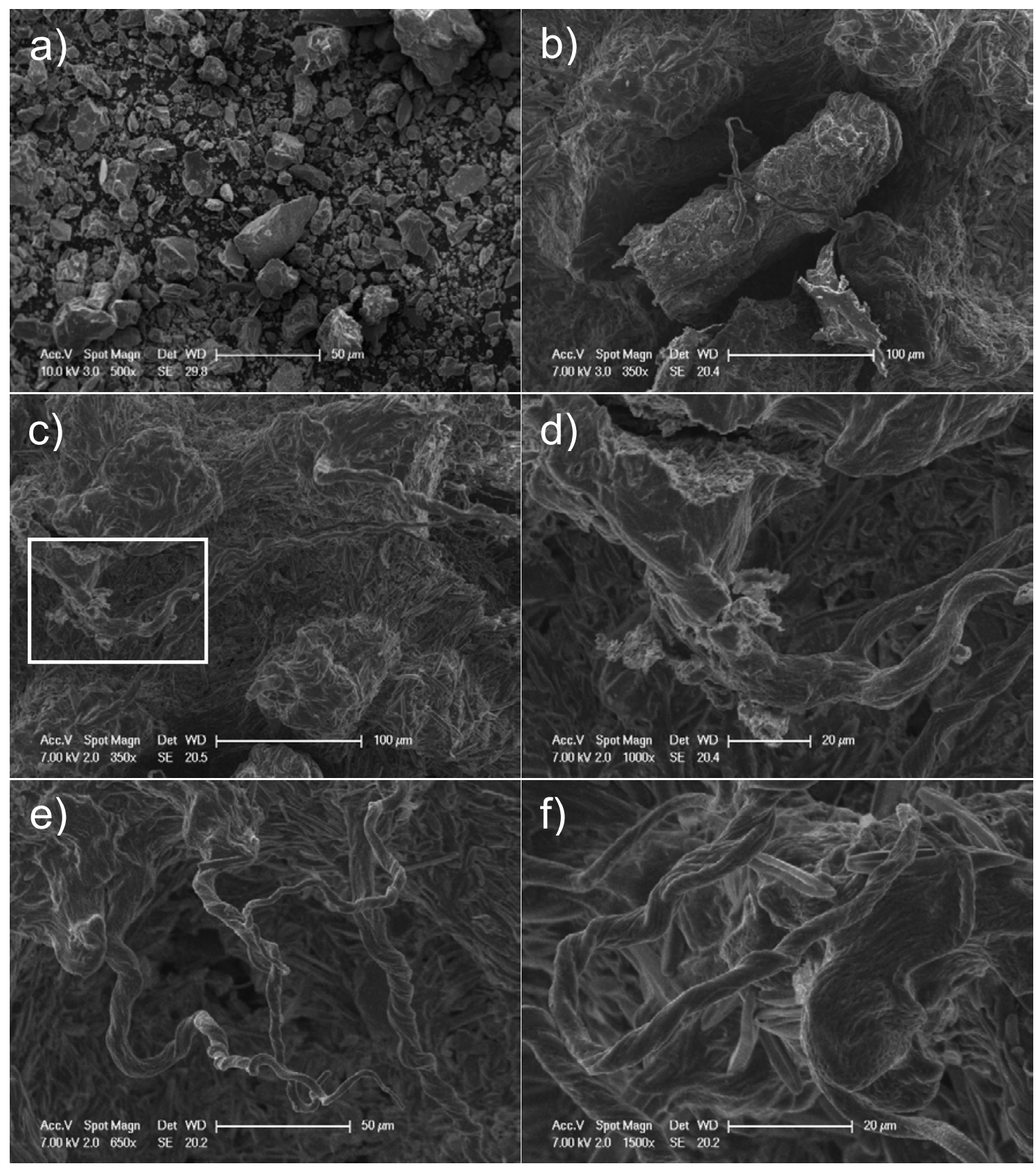

Figure 6-11 | Scanning electron microscopy of (a) untreated magnetite tailings, and (b-f) Eagle River crust on MI/MN blend tailings. (a) The untreated tailings particles are angular and do not feature any notable coating. (b) A visible coating, presumed to be EPS, can be observed on the surface of the tailings, as well as some rod-shaped organisms and filaments. (c) The same visible EPS coating can be seen, as well as some rod-shaped organisms and multiple, intertwined filaments; the connection of a filament to the crust/tailings surface, filament branching, and particle attachment to the filament are highlighted in the rectangle and can be seen in more detail in (d). (e) Filament connections to the crust/tailings surface and intertwined filaments are shown. (f) Zooming in shows additional detail, including pores in the filaments and crust. 


\subsubsection{Bioreactor Freezing and Sublimation}

Images demonstrated how ER bioreactors were affected by freezing/sublimation are shown in Figure 6-12. Importantly, the crusts remained intact during freezing (Figure 612(a-b)). Below the crusts, the tailings behaved as untreated samples are expected to behave, as documented by Zwissler et al. (in preparation). Specifically, the tailings expanded vertically, ice lenses were formed, with some of the ice in the ice lenses sublimating, and the surface showing color change indicative of the freezing/sublimation process (Figure 6-12(c-d)).
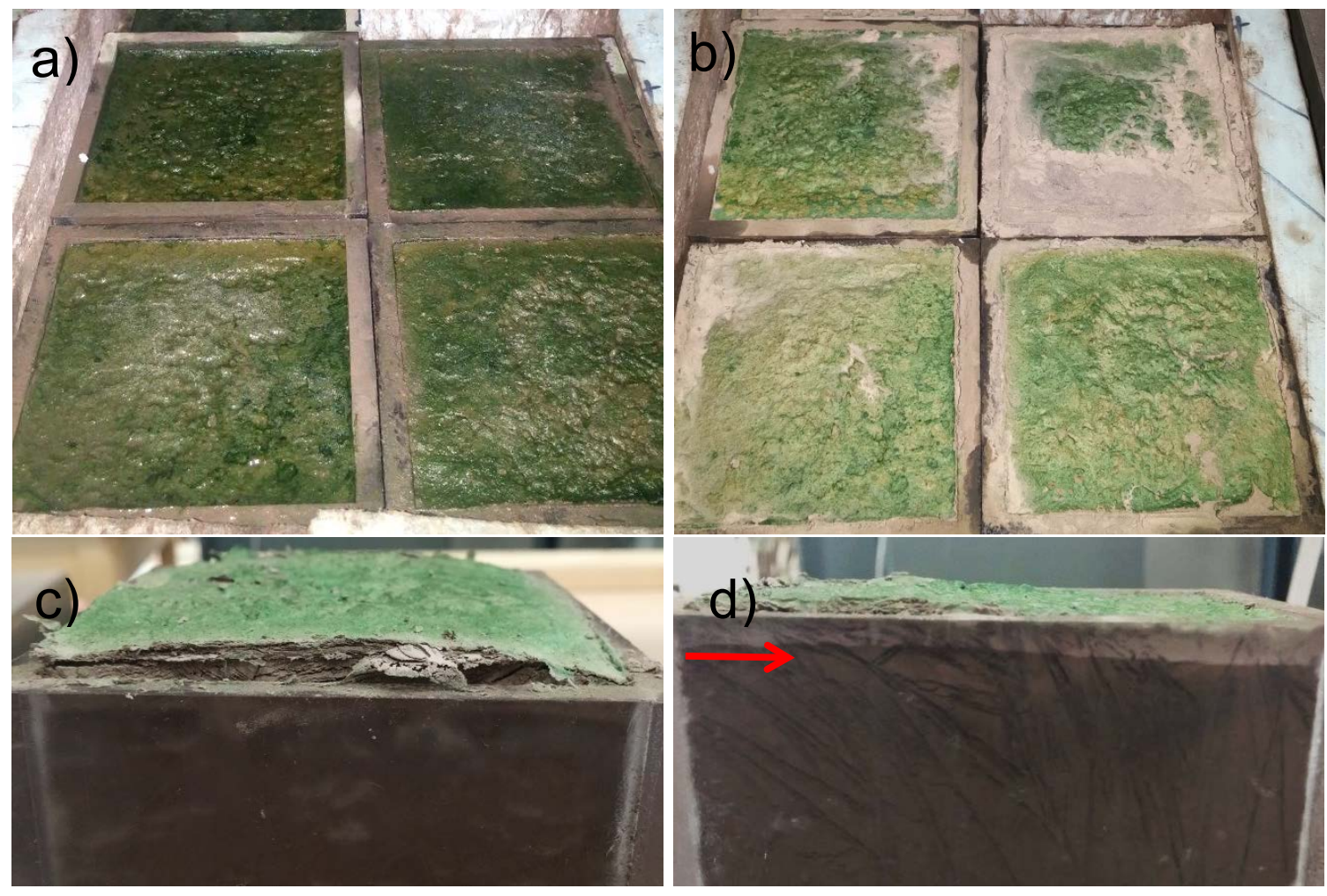

Figure 6-12 | Eagle River bioreactors (a) before freezing, (b) after freezing, and (c-d) after removal from freezer. (a) and (b) show that freezing and sublimation does not cause damage to the crust surface; the back right sample pictured in (b) was covered by underlying tailings during sample saturation, but crust is intact underneath thin tailings layer. (c) and (d) show that the underlying tailings mimic freezing behavior, ice lens formation, and sublimation (with the depth of sublimation clearly defined by the tailings color change in (d)) of untreated tailings. 


\subsubsection{Wind Tunnel Testing}

Before examining the performance of the bioreactors in the wind tunnel, it is important to understand what the baseline, or untreated, conditions are. Due to unavoidable inconsistencies in sample conditions, not all samples freeze in the same manner and, therefore, the samples prepared as described in this work experience different quantities of vertical expansion due to ice lens generation. Zwissler et al. (in preparation) found that mass lost during wind tunnel testing is a function of sample vertical expansion. Therefore, the same framework was used for presenting the wind tunnel results in this work, with the results from the wind tunnel testing of the BG-11 controls and ER bioreactors presented in Figure 6-13 and Figure 6-14, respectively. In these two figures, any treated sample that falls below the trendline for the untreated tailings is interpreted as having resisted wind erosion more effectively than untreated tailings.

Because the control bioreactors still were treated with BG-11 growth medium, their behavior was not automatically assumed to mimic the untreated tailings baseline data previously generated by Zwissler et al. (in preparation). However, as shown in Figure 6-14, the BG-11 control bioreactors tested in the wind tunnel have similar trends to the untreated tailings samples. Thus, the treatment of BG-11 growth medium alone did not alter the behavior of the bioreactors in the wind tunnel.

The results for the wind tunnel testing of the ER bioreactors were not as clear-cut (Figure 6-14). Four of the wind tunnel tests on ER bioreactors were successful and clearly demonstrated the benefit of having a crust (bioreactor data points below the trendline in Figure 6-14). During wind tunnel testing of these bioreactors, no tailings originating from under the crust were observed to be lost, so any mass lost was due to loose material from the edges disturbed during transport or from small layers of tailings deposited on top of the crust. For these samples, the crust itself showed no signs of fatigue or distress due to this wind tunnel testing, and likely could have withstood higher speeds and longer wind durations if sample melting was not a concern, as discussed below. 
However, the remaining four wind tunnel tests conducted on ER bioreactors failed in a way that did not accurately represent wind erosion conditions that would be acting on a tailings impoundment (bioreactor data points above the trendline in Figure 6-14). Three of the four failed tests had imperfections in the leading edge of the crust (either slight curling or voids present under the crust due to ice lens sublimation), which allowed the wind to rip off the crust. The last of the failed ER tests was a sample that had a layer of tailings deposited on top of the crust surface due to the sample saturation technique used in freezing (a more extreme version of the crust coverage shown in Figure 6-12b). Thus, in all four cases, the failed samples behaved in ways that confounded the results, and prevented their comparison with the baseline data.

Nevertheless, the four failed ER bioreactor wind tunnel tests are also important to assess, because the ways that these samples failed demonstrates key differences between laboratory testing and the way the crusts would act in the field. For example, the one ER bioreactor that had high mass lost due to removal of tailings deposited on top of the crust is not representative of field conditions, because it is unlikely that a thin layer of tailings would be deposited on the surface of a crust. The three failed ER bioreactors that had the crust removed during wind tunnel testing highlight one of the largest limitations of this study, which was the sample size. There are many reasons why a relatively small sample size was chosen, including tailings and inoculum resource limitations, repeatability, freezer space, and ease of sample transport. However, for wind tunnel testing, a larger sample surface area would have been preferable. With a larger surface area, edge effects would have been less significant. Furthermore, with a larger surface area, the impacts of sealing the crust edges (e.g., McKenna Neuman et al. 1996) would be much smaller, allowing larger samples with edge imperfections to still be tested.

In addition to the limitations of laboratory wind tunnel testing due to sample size, there are also limitations with regards to the laboratory temperature. When tailings impoundments experience cold-weather dusting, ambient temperatures are close to $0^{\circ} \mathrm{C}$; this cold wind passing over the surface of the tailings does not cause sample melting, and therefore the wind can cause additional sublimation to generate dust from the tailings 
surface. However, the wind tunnel used in this study is located in a laboratory kept at standard room temperatures, which means that the air passing across the sample surface caused rapid melting within 1 minute of exposure. As a result, the samples were only placed in the wind tunnel for 30 seconds. Wind tunnel testing would likely yield more dramatic results demonstrating the wind erosion resistance of BSCs like the ER bioreactors if the wind passing across their surface was at or below freezing temperatures, allowing wind tunnel tests to run for longer periods of time.

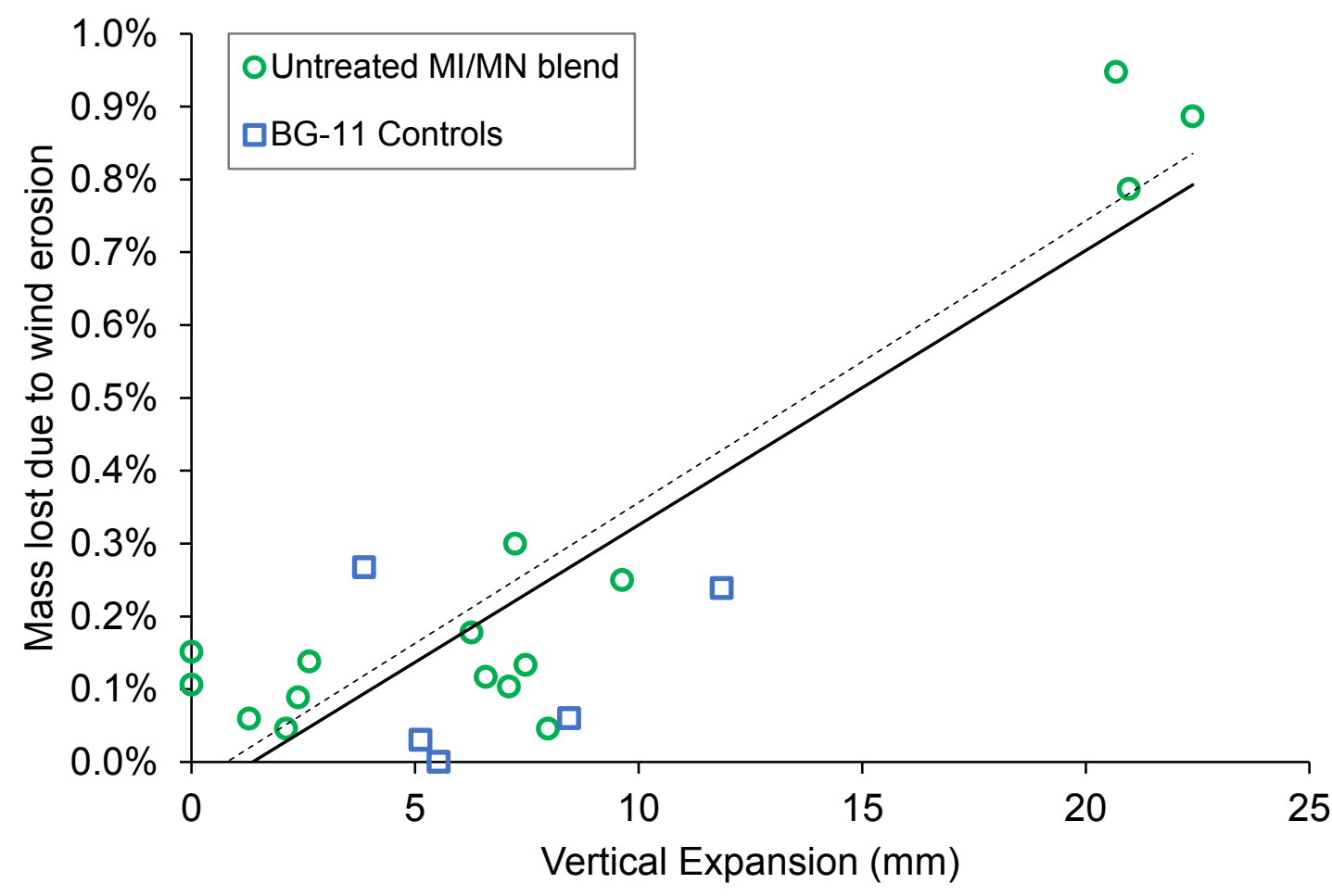

Figure 6-13 | Wind tunnel results for untreated MI/MN blend tailings (Zwissler et al. in preparation) and for BG-11 control bioreactors (this work). The trends of the BG-11 control bioreactors agree with the trends observed for the untreated MI/MN blend tailings (dashed line, $\mathrm{R}^{2}=0.86$ ), indicating that the datasets can be combined to establish baseline conditions (solid line, $\mathrm{R}^{2}=0.77$ ). Each point represents results for a single sample. 


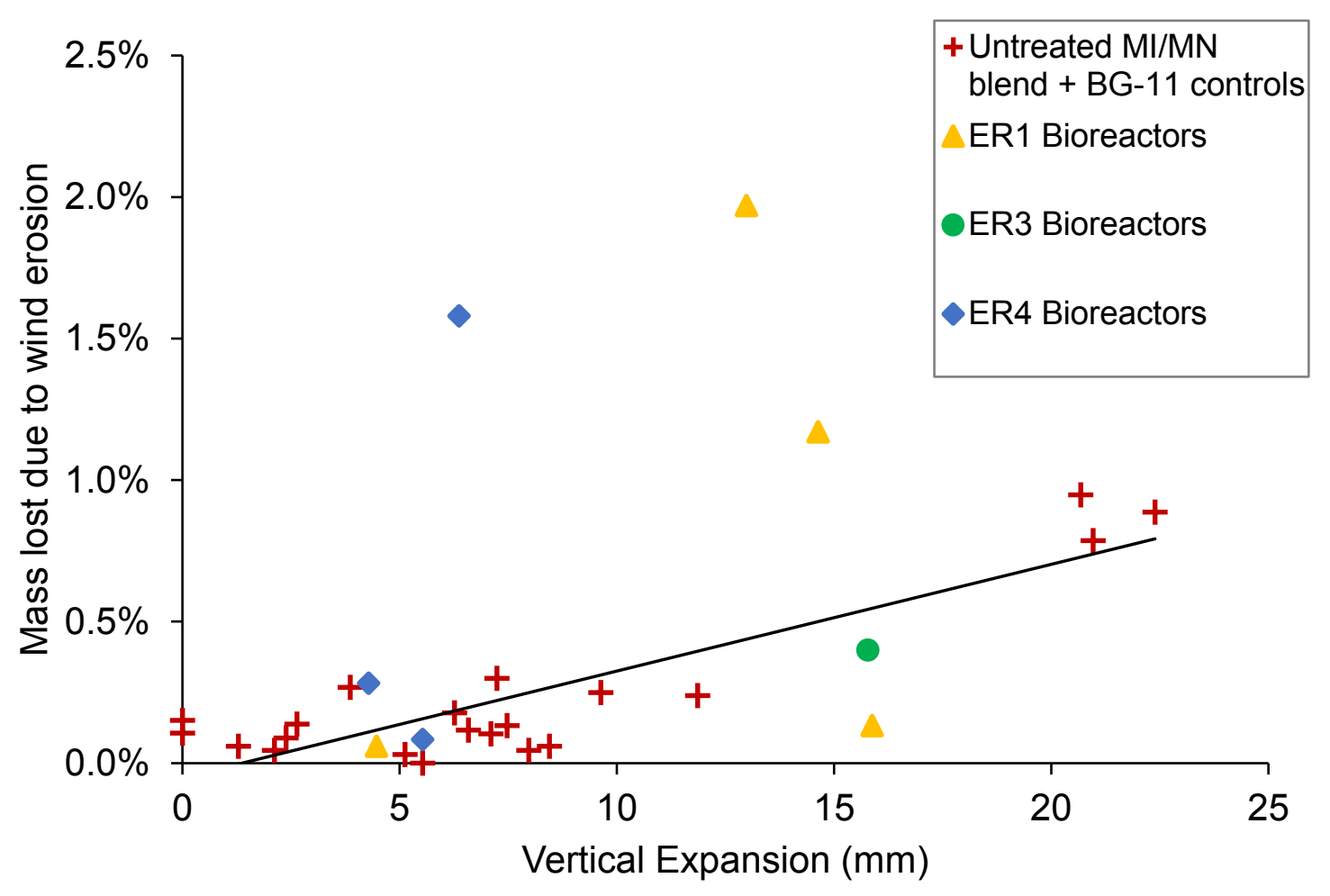

Figure 6-14 | Wind tunnel results for ER bioreactors and compared to the baseline conditions of untreated MI/MN blend tailings (Zwissler et al. in preparation) and for BG11 control bioreactors. All ER bioreactors with mass lost falling below the trendline are interpreted to resist wind erosion better than untreated tailings. All ER bioreactors with mass lost above the trendline failed due to conditions unrepresentative of field conditions. Each point represents results for a single sample.

\subsubsection{Ball Drop Testing}

The results from the ball drop testing of the ER and BG-11 bioreactors are presented in Figure 6-15 and Figure 6-16, respectively. As for the wind tunnel data, these results are compared to the results from a suite of ball drop tests performed on untreated $\mathrm{MI} / \mathrm{MN}$ blend tailings, previously published by Zwissler et al. (in preparation). Based on the work by Zwissler et al. (in preparation), the baseline data for the ball drop indicates that impact crater diameter is also a function of sample vertical expansion due to freezing, which is how the initial ball drop results are plotted. Any treated sample that falls below the trendline for untreated tailings is interpreted to resist impact (e.g. from saltating particles) more effectively than untreated tailings and have higher surface strength. 
Similar to the wind tunnel testing, the behavior of the control bioreactors treated with BG-11 growth medium was not automatically assumed to mimic the untreated baseline data previously generated. However, as shown in Figure 6-15, the BG-11 control bioreactors exhibited similar trends to the untreated tailings samples with respect to vertical expansion and impact crater diameter. Thus, the treatment of BG-11 growth medium alone did not alter the behavior of the bioreactors in resisting ball bearing impact. Note that the trendline through the data in Figure 6-15 is relatively flat; however, the slope was still found to be statistically significant ( 2 tailed t-test, $\alpha=0.05$ ).

The ball drop data for the ER bioreactors, shown in Figure 6-16, clearly demonstrates the increase in surface strength and impact resistance provided by the presence of a crust. All ER bioreactors fall below the untreated trendline, indicating that the presence of a crust helps to resist impact. While some crusts did rupture upon impact, a large number of crusts had at least some of the impacts resisted by the crust. All bioreactors with a crater diameter of zero had a crust that absorbed every impact and rebounded without leaving behind a crater.

Thus, the ER crusts were able to act as a flexible membrane (Otto and Trostel 1962) to absorb impacts, and were often able to fully rebound without leaving behind a crater. The ability to resist the forces applied during this ball drop test indicate that the crust could sustain significant abrasion from saltating particles (if present) during potential wind erosion events. 


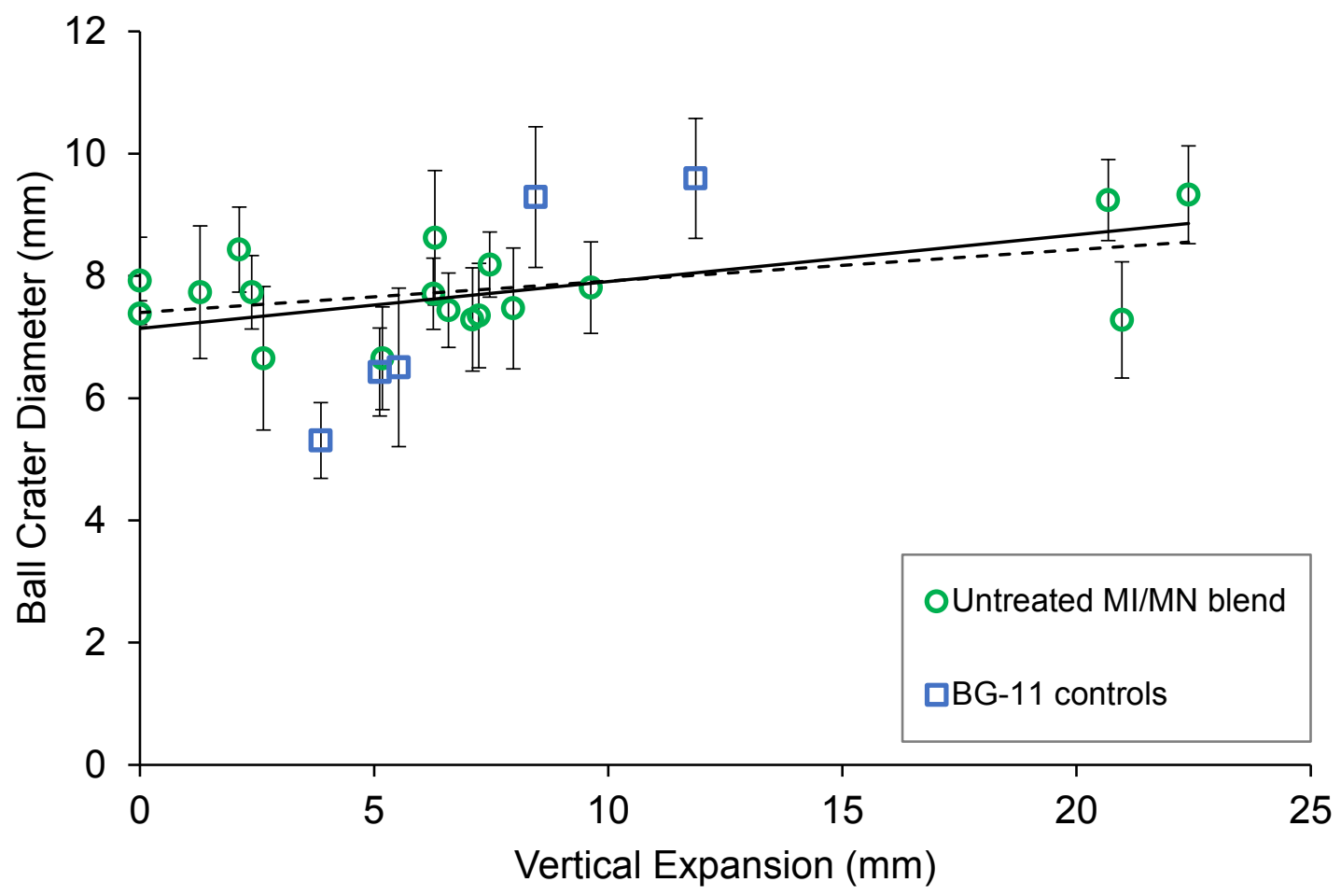

Figure 6-15 | Baseline ball drop results for untreated MI/MN blend tailings (Zwissler et al. in preparation) and for BG-11 control bioreactors. The trends of the BG-11 control bioreactors agree with the trends observed for the untreated MI/MN blend tailings (dashed line, $\mathrm{R}^{2}=0.23$ ), indicating that the datasets can be combined to establish baseline conditions (solid line, $\mathrm{R}^{2}=0.21$ ). Each point represents the average diameter of 5 craters, and error bars represent standard deviation. 


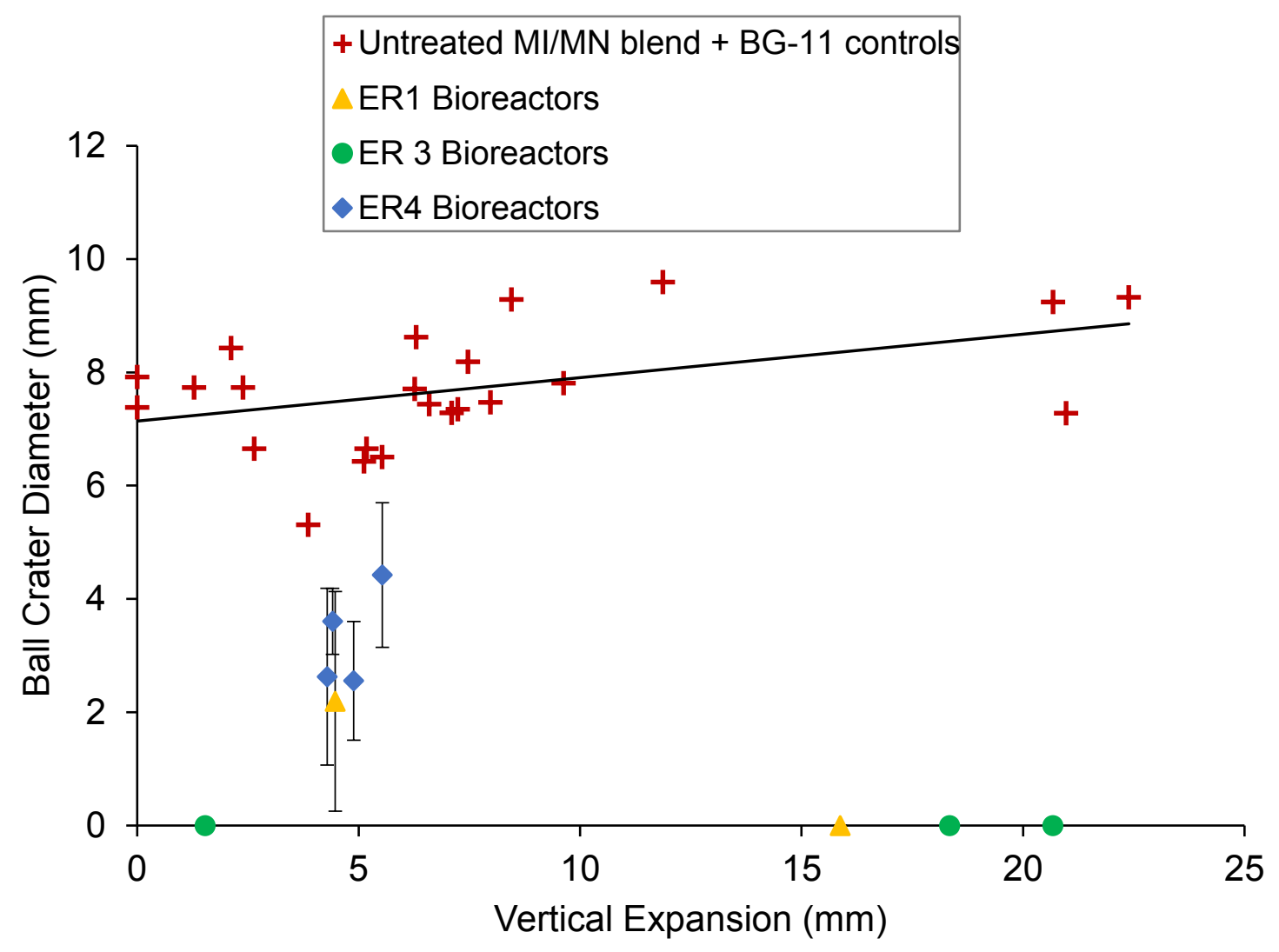

Figure 6-16 | Ball drop results for ER bioreactors compared to untreated MI/MN blend tailings (Zwissler et al. in preparation) and BG-11 control bioreactors. All bioreactors fall below the untreated trendline, indicating that the presence of a crust helps to resist impact. All bioreactors with a crater diameter of zero had a crust that absorbed the impact and rebounded without leaving behind a crater. Each point represents the average diameter of 5 craters, and error bars represent standard deviation.

\subsection{Practical Applications}

Dust emissions from fine grained sediment sources, like tailings impoundment, pose significant environmental- and human-health hazards. In cold regions, much of the concern with respect to dusting is focused on sublimation-driven cold weather dust emissions. Tailings impoundment managers for mining activities in cold regions are actively aware of these hazards, and invest heavily in treating their impoundments to resist dust. Rather than having to rely on chemical and physical additives, this research 
suggests that BSCs from locally-sourced organisms may be a suitable alternative dust mitigation treatment for tailings impoundment managers to employ. The laboratory crusts produced in this study showed the ability to withstand freezing and significant erosion resistance potential.

Much additional work is needed to determine the feasibility of full scale application of this research, including cost estimates, optimal inoculum density, and treatment logistics. Nevertheless, this research is the first step in the development of using BSCs as an environmentally-friendly dust suppression treatment.

\subsection{Conclusions}

The goal of this study was to examine the use of BSCs for decreasing dust emissions from mine tailings exposed to cold-weather dusting conditions. This was assessed via laboratory-scale testing of tailings samples biologically treated using a locally-sourced, primarily filamentous cyanobacteria inoculum as a pioneer organism for a BSC to develop. Laboratory samples were monitored with remote sensing during their development, characterized for biomass concentration, assessed with SEM, and tested for erosion resistance using wind tunnel and ball drop testing. This study showed that:

- BSCs can be cultivated on mine tailings, specifically crusts inoculated with locally sourced organisms; however, not all organisms are suitable for BSC development on tailings, as shown by the lack of development of the pure culture A. dololium crusts.

- Remote sensing using NDVI is an appropriate method for monitoring the biomass development of phototrophic BSCs $\left(\mathrm{R}^{2}=0.85\right.$ for chlorophyll a).

- The unidentified Eagle River organisms used for BSC inoculum excreted measurable EPS (between 15 and $19 \mu \mathrm{g} / \mathrm{g}$ for healthy crusts) during crust development. SEM imagery verifies the production of EPS, as well as the presence of filaments, that interacted with the tailings/crust surface 
- The BSCs produced in the laboratory remained intact during freezing, and after freezing were able to resist wind erosion and increase surface strength, as measured by wind tunnel and ball drop testing,

- The performance of these Eagle River crusts during wind tunnel and ball drop testing demonstrated the potential advantages of having a flexible membrane as a surface cover to resist erosion; no tailings were removed from under the crust during wind tunnel tests, and the ball bearing bounced off the crust surface, often without leaving an impact crater behind.

\subsection{References}

Ashley, J., and Rushforth, S. (1984). "Growth of soil algae on top soil and processed oil shale from the Uintah Basin, Utah, USA." Reclamation \& revegetation research, 3(1), 49-63.

Association, A. P. H. (1994). "Water Environment Federation (1998) Standard methods for the examination of water and wastewater." Washington, Dc.

Bang, S. C., and Bang, S. S. (2011). "KGS Awards Lectures: Application of Microbiologically Induced Soil Stabilization Technique for Dust Suppression." International Journal of Geo-Engineering, 3(2), 27-37.

Bang, S. S., Bang, S., Frutiger, S., Nehl, L. M., and Comes, B. L. "Application of novel biological technique in dust suppression." Proc., Transportation Research Board 88th Annual Meeting.

Belnap, J. (1993). "Recovery rates of cryptobiotic crusts: inoculant use and assessment methods." Western North American Naturalist, 53(1), 89-95.

Belnap, J. (1995). "Surface disturbances: Their role in accelerating desertification." Environmental monitoring and assessment, 37(1-3), 39-57.

Belnap, J., and Eldridge, D. (2003). "Disturbance and recovery of biological soil crusts." Biological soil crusts: structure, function, and management, Springer, 363-383.

Belnap, J., and Gardner, J. S. (1993). "Soil microstructure in soils of the Colorado Plateau: the role of the cyanobacterium Microcoleus vaginatus." Western North American Naturalist, 53(1), 40-47.

Belnap, J., and Gillette, D. (1997). "Disturbance of biological soil crusts: impacts on potential wind erodibility of sandy desert soils in southeastern Utah." Land Degradation \& Development, 8(4), 355-362.

Belnap, J., and Gillette, D. A. (1998). "Vulnerability of desert biological soil crusts to wind erosion: the influences of crust development, soil texture, and disturbance." Journal of Arid Environments, 39(2), 133-142.

Belnap, J., Laxalt, M., and Peterson, P. (2001). Biological soil crusts: ecology and management, US Department of the Interior, Bureau of Land Management, 
National Science and Technology Center, Information and Communications Group.

Buck, S., and Gerard, D. (2001). "Cleaning Up Mining Waste." Political Economy Research Center Research Study.

Buikema, N. D. (2015). "Stabilization of iron mine tailings through microbially induced calcite precipitation."

Buikema, N. D., Zwissler, B. E., Seagren, E. A., Oommen, T., and Vitton, S. J. (accepted). "Stabilization of Iron Mine Tailings through Biocalcification." Environmental Geotechnics.

Cameron, R. E., and Blank, G. B. (1966). "Desert algae: soil crusts and diaphanous substrata as algal habitats."

Colica, G., Li, H., Rossi, F., De Philippis, R., and Liu, Y. (2015). "Differentiation of the characteristics of excreted extracellular polysaccharides reveals the heterogeneous primary succession of induced biological soil crusts." Journal of Applied Phycology, 27(5), 1935-1944.

De Philippis, R., Sili, C., Paperi, R., and Vincenzini, M. (2001). "Exopolysaccharideproducing cyanobacteria and their possible exploitation: a review." Journal of Applied Phycology, 13(4), 293-299.

DeJong, J., Soga, K., Kavazanjian, E., Burns, S., AL QABANY, A., AYDILEK, A., BANG, S., BURBANK, M., CASLAKE, L., and CHEN, C. (2013).

"Biogeochemical processes and geotechnical applications: progress, opportunities and challenges." Geotechnique, 63(4), 287-301.

Dubois, M., Gilles, K. A., Hamilton, J. K., Rebers, P., and Smith, F. (1956).

"Colorimetric method for determination of sugars and related substances." Analytical chemistry, 28(3), 350-356.

Eldridge, D., and Greene, R. (1994). "Microbiotic soil crusts-a review of their roles in soil and ecological processes in the rangelands of Australia." Soil Research, 32(3), 389-415.

Eldridge, D. J., and Leys, J. F. (2003). "Exploring some relationships between biological soil crusts, soil aggregation and wind erosion." Journal of Arid Environments, 53(4), 457-466.

Friedmann, E. I., and Galun, M. (1974). "Desert algae, lichens and fungi." Desert biology, 2, 165-212.

Friedmann, E. I., and Ocampo-Paus, R. (1976). "Endolithic blue-green algae in the Dry Valley: primary producers in the Antarctic Desert ecosystem." Science, 193, 1247-1249.

Garcia-Pichel, F., and Castenholz, R. W. (1991). "Characterization and Biological Implications of Scytonemin, a Cyanobacterial Sheath Pigment1." Journal of Phycology, 27(3), 395-409.

Harper, K. T., and Marble, J. R. (1988). "A role for nonvascular plants in management of arid and semiarid rangelands." Vegetation science applications for rangeland analysis and management, Springer, 135-169.

Huang, L. N., Tang, F. Z., Song, Y. S., Wan, C. Y., Wang, S. L., Liu, W. Q., and Shu, W. S. (2011). "Biodiversity, abundance, and activity of nitrogen-fixing bacteria 
during primary succession on a copper mine tailings." FEMS microbiology ecology, 78(3), 439-450.

Leys, J. F., and Eldridge, D. J. (1998). "Influence of cryptogamic crust disturbance to wind erosion on sand and loam rangeland soils." Earth Surf. Process. Landf., 23(11), 963-974.

Lindahl, V., and Bakken, L. R. (1995). "Evaluation of methods for extraction of bacteria from soil." FEMS Microbiology Ecology, 16(2), 135-142.

Liu, Y., Cockell, C. S., Wang, G., Hu, C., Chen, L., and De Philippis, R. (2008). "Control of Lunar and Martian dust-experimental insights from artificial and natural cyanobacterial and algal crusts in the desert of Inner Mongolia, China." Astrobiology, 8(1), 75-86.

Lukešová, A. (2001). "Soil Algae in Brown Coal and Lignite Post-Mining Areas in Central Europe (Czech Republic and Germany)." Restoration Ecology, 9(4), 341350 .

McKenna Neuman, C. (1990). "Role of sublimation in particle supply for aeolian transport in cold environments." Geografiska Annaler. Series A. Physical Geography, 329-335.

McKenna Neuman, C., and Maxwell, C. (1999). "A wind tunnel study of the resilience of three fungal crusts to particle abrasion during aeolian sediment transport." Catena, 38(2), 151-173.

McKenna Neuman, C., and Maxwell, C. (2002). "Temporal aspects of the abrasion of microphytic crusts under grain impact." Earth Surf. Process. Landf., 27(8), 891908.

McKenna Neuman, C., Maxwell, C., and Rutledge, C. (2005). "Spatial and temporal analysis of crust deterioration under particle impact." Journal of Arid Environments, 60(2), 321-342.

McKenna Neuman, C., Maxwell, C. D., and Boulton, J. W. (1996). "Wind transport of sand surfaces crusted with photoautotrophic microorganisms." Catena, 27(3), 229-247.

Meyer, F., Bang, S., Min, S., Stetler, L., and Bang, S. (2011). "Microbiologically-induced soil stabilization: application of Sporosarcina pasteurii for fugitive dust control." Proceedings of Geo-Frontiers, 4002-4011.

Meyer, H. A., Lyons, A. M., Nelson, D. R., and Hinton, J. G. (2011). "Tardigrada of Michigan, Northern USA, with the description of Minibiotus jonesorum sp. n.(Eutardigrada: Macrobiotidae)." Journal of Zoological Systematics and Evolutionary Research, 49(s1), 40-47.

O'Brien, P., and McKenna Neuman, C. (2012). "A wind tunnel study of particle kinematics during crust rupture and erosion." Geomorphology, 173, 149-160.

Orlekowsky, T., Venter, A., van Wyk, F., and Levanets, A. (2013). "Cyanobacteria and algae of gold mine tailings in the Northwest Province of South Africa." Nova Hedwigia, 97(3-4), 281-294.

Otto, F., and Trostel, R. (1962). "Tensile Structures, Vol. I." Massachusetts institute of Technology, Cambridge. 
Pérez, F. L. (1997). "Microbiotic crusts in the high equatorial Andes, and their influence on paramo soils." Catena, 31(3), 173-198.

Schlichting, H. E., Jr. (1969). "The importance of airborne algae and protozoa." Journal of the Air Pollution Control Association, 19(12), 946-951.

Shubert, L. E., and Starks, T. L. (1980). "Soil-algal relationships from surface mined soils." British Phycological Journal, 15(4), 417-428.

Stanier, R., Kunisawa, R., Mandel, M., and Cohen-Bazire, G. (1971). "Purification and properties of unicellular blue-green algae (order Chroococcales)." Bacteriological reviews, 35(2), 171.

Stovern, M., Betterton, E. A., Sáez, A. E., Villar, O. I. F., Rine, K. P., Russell, M. R., and King, M. (2014). "Modeling the emission, transport and deposition of contaminated dust from a mine tailing site." Reviews on environmental health, 29(1-2), 91-94.

Sun, Q., An, S., Yang, L., and Wang, Z. (2004). "Chemical properties of the upper tailings beneath biotic crusts." Ecological Engineering, 23(1), 47-53.

Thenkabail, P. S., Lyon, J. G., and Huete, A. (2011). Hyperspectral remote sensing of vegetation, CRC Press.

Thiet, R. K., Boerner, R., Nagy, M., and Jardine, R. (2005). "The effect of biological soil crusts on throughput of rainwater and N into Lake Michigan sand dune soils." Plant and soil, 278(1-2), 235-251.

Thomas, A. D., and Dougill, A. J. (2007). "Spatial and temporal distribution of cyanobacterial soil crusts in the Kalahari: implications for soil surface properties." Geomorphology, 85(1), 17-29.

USEPA (2012). "Particulate Matter (PM-10)." $<$ http://www.epa.gov/airtrends/aqtrnd95/pm10.html $>$.

USEPA (2014). "Fine Particule Matter (PM2.5) Designations." $<$ http://www.epa.gov/pmdesignations/>.

Vick, S. G. (1983). Planning, Design, and Analysis of Tailings Dams, John Wiley \& Sons, Inc., New York, NY.

West, N. E. (1990). "Structure and functions of microphytic soil crusts in wildland ecosystems of arid to semi-arid regions." Advances in ecological Research, 20, $179-223$.

Williams, J., Dobrowolski, J., West, N., and Gillette, D. (1995). "Microphytic crust influence on wind erosion." Transactions of the ASAE, 38(1), 131-137.

Zaady, E., Ben-David, E. A., Sher, Y., Tzirkin, R., and Nejidat, A. (2010). "Inferring biological soil crust successional stage using combined PLFA, DGGE, physical and biophysiological analyses." Soil Biology and Biochemistry, 42(5), 842-849.

Zwissler, B. E., Vitton, S. J., Seagren, E. A., and Oommen, T. (in preparation). "Laboratory method to generate dust via sublimation to study cold weather dusting phenomenon for fine-grained sediments." 



\section{Chapter 7 Synthesis of Research and Broader Impacts}

The overall goal of this research was to develop and evaluate effective, economical, and sustainable solutions to two major issues facing mine tailings impoundments with regards to dusting: (1) dust monitoring/detection and (2) dust reduction/prevention. To achieve this goal, laboratory and field testing was used to assess the utility of thermal remote sensing techniques for dust monitoring, and laboratory testing was also used to assess the ability of biological soil crusts to reduce dust emissions due to cold weather dusting.

A laboratory model was developed to use thermal remote sensing and other atmospheric variables to predict surface moisture content and strength of iron mine tailings. Although this laboratory model was not found to be directly applicable to field remote sensing, this research suggests that a model could be developed using field data to predict surface moisture content using thermal remote sensing, which would be a useful tool for tailings impoundment managers to employ for dust emissions detection. For such a model to be useful to tailings impoundment managers, thermal remote sensing would need to be collected with appropriate spatial and temporal resolutions and coverage. The most likely way for this data to be collected is with a UAV-based platform. UAV technology is rapidly developing, with UAVs becoming more affordable and sensors (including thermal sensors) becoming more lightweight and UAV-friendly. The UAV industry is booming, and the application of this research demonstrates one of many ways that UAVs can be used in the future (Liu et al. 2014). For the application of a field based model to predict surface moisture and strength of tailings with thermal remote sensing, the only requirements are to have a UAV that can carry the payload of a thermal sensor and a means image mosaic generation and georeferencing.

Additionally, a method was developed and validated that exposed laboratory tailings sample to freezing and sublimation conditions that are representative of those experienced at tailings impoundments located in cold-weather climates, and the dust emissions and strength of these tailings samples was characterized using a wind tunnel 
and ball drop testing. The development of these laboratory methods is meaningful, because they provide a way to enable the study of the coupling of the phenomena of ice lens generation, sublimation, and dust emissions for fine grained sediments like mine tailings. Utilization of these methods could greatly help the understanding of these complex processes and the factors that impact ice lens formation, dust generation via sublimation, and dust emissions for fine grained sediments. Beyond the understanding of how tailings and other fine grained sediments are affected by freezing and sublimation, the application of these lab methods could easily be used to assess the effectiveness of dust suppressants. There are a number of commercially available chemical and physical dust suppressants available, and studying how these different treatments hold up to sublimation would provide useful information to tailings impoundment managers trying to prevent cold weather dusting. Also, there is a recent push in research to develop novel dust suppressants that have a lower environmental impact (Buikema et al. accepted; Hamdan and Kavazanjian Jr 2016). Especially when developing new dust suppressants that are aimed at helping resist cold-weather dusting, being able to test the treatment's resistance to sublimation at laboratory scales prior to pilot scale tests would help researchers greatly. Specifically, the effectiveness of treatments could be assessed by comparing wind tunnel and strength test results for treated samples to results for untreated tailings. Using these methods would provide a systematic way to assess the potential of novel dust suppressants in-development that are being designed to resist sublimation-induced dusting, to inform researchers to develop better products.

Lastly, biological soil crusts originating from locally-sourced organisms were grown on laboratory tailings samples, and when exposed to freezing/sublimation and tested with wind tunnel and ball drop testing, the biological soil crusts were found to provide resistance to wind erosion and increased surface strength. Sublimation-driven cold weather dust emissions are a significant, yet often forgotten, potential dust source, yet tailings impoundment managers for mining activities in cold regions are actively aware of these hazards, and invest heavily in treating their impoundments to resist dust. Rather than having to rely on chemical and physical additives, this research suggests that 
biological soil crusts from locally-sourced organisms may be a suitable alternative dust mitigation treatment for tailings impoundment managers to employ. The laboratory crusts produced in this study showed the ability to withstand freezing and significant erosion resistance potential. Much additional work is needed to determine the feasibility of full scale application of this research, including cost estimates, optimal inoculum density, and treatment logistics. Nevertheless, this research is the first step in the development of using biological soil crusts as an environmentally-friendly dust suppression treatment.

These results are important, both for the understanding of dust emissions and potential dust mitigation treatments for tailings impoundments, and also for broader issues of wind erosion and dust emissions of soil both globally and on other planets. The remote sensing principles used in this research can also be applied to other geotechnical applications that require surficial moisture content and/or strength characterization and monitoring, such as trafficability monitoring for agricultural and military purposes. However, for the techniques utilized in this research to be applied to other soils, a new laboratory calibration between thermal remote sensing and surface strength would need to be developed for each soil. These techniques would also be limited to un-vegetated conditions. Biological soil crusts can be used in a variety of applications besides just dust suppression and strength increase for tailings. Across the planet, biological soil crusts have demonstrated their utility for stabilizing soils in arid environments where little to no vascular vegetation can grow. In new or existing areas that are affected by arid conditions or a lack of vegetation, biological soil crusts could be stimulated to reduce erosion susceptibility. For example, one potential application of biological soil crust cultivation would be for dust control in enclosed spaces during space exploration on the Moon, Mars, and other planets (Liu et al. 2008).

\subsection{References}

Buikema, N. D., Zwissler, B. E., Seagren, E. A., Oommen, T., and Vitton, S. J. (accepted). "Stabilization of Iron Mine Tailings through Biocalcification." Environmental Geotechnics.

Hamdan, N., and Kavazanjian Jr, E. (2016). "Enzyme-induced carbonate mineral precipitation for fugitive dust control." Géotechnique, 66(7), 546-555. 
Liu, P., Chen, A. Y., Huang, Y.-N., Han, J.-Y., Lai, J.-S., Kang, S.-C., Wu, T.-H., Wen, M.-C., and Tsai, M.-H. (2014). "A review of rotorcraft Unmanned Aerial Vehicle (UAV) developments and applications in civil engineering." SMART STRUCTURES AND SYSTEMS, 13(6), 1065-1094.

Liu, Y., Cockell, C. S., Wang, G., Hu, C., Chen, L., and De Philippis, R. (2008). "Control of Lunar and Martian dust-experimental insights from artificial and natural cyanobacterial and algal crusts in the desert of Inner Mongolia, China." Astrobiology, 8(1), 75-86. 